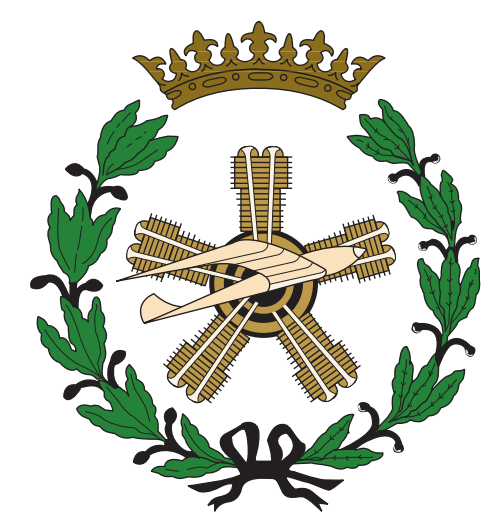

UNIVERSIDAD POLITÉCNICA DE MADRID

ESCUELA TÉCNICA SUPERIOR DE INGENIEROS AERONÁUTICOS

\title{
ESTUDIO DE UN SISTEMA DE GENERACIÓN INDUSTRIAL DE POTENCIA PARA CERO \\ EMISIONES DE $\mathrm{CO}_{2}$
}

TESIS DOCTORAL

Vicente Paúl Timón Piote

Ingeniero aeronáutico 

DEPARTAMENTO DE MECÁNICA DE FLUIDOS Y PROPULSIÓN AEROESPACIAL

ESCUELA TÉCNICA SUPERIOR DE INGENIEROS AERONÁUTICOS

\title{
ESTUDIO DE UN SISTEMA DE GENERACIÓN INDUSTRIAL DE POTENCIA PARA CERO
} EMISIONES DE $\mathrm{CO}_{2}$

\author{
Autor: \\ Vicente Paúl Timón Piote \\ Ingeniero aeronáutico \\ Directores: \\ Gregorio Corchero Díaz \\ Doctor ingeniero aeronáutico \\ José Luis Montañés García \\ Doctor ingeniero aeronáutico
}





\section{POLITÉCNICA}

Tribunal nombrado por el Sr. Rector Magfco. de la Universidad Politécnica de Madrid, el día de de $20 \ldots$

Presidente:

Vocal:

Vocal:

Vocal:

Secretario:

Suplente:

Suplente:

Realizado el acto de defensa y lectura de la Tesis el día de. de $20 \ldots$ en la E.T.S.I. /Facultad.

Calificación

EL PRESIDENTE

LOS VOCALES 



\section{ÍNDICE.}

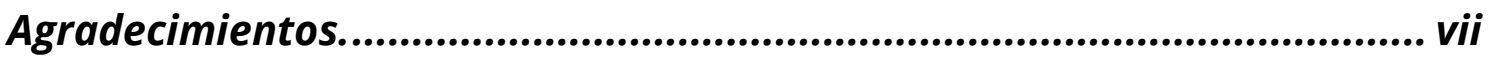

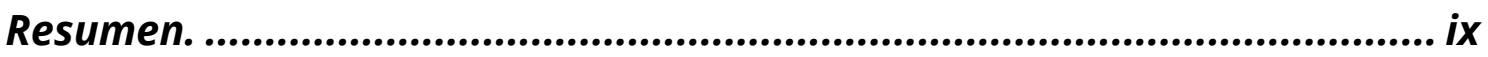

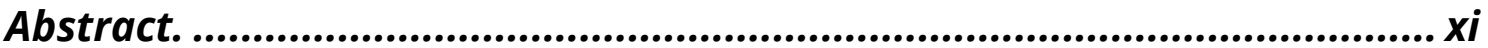

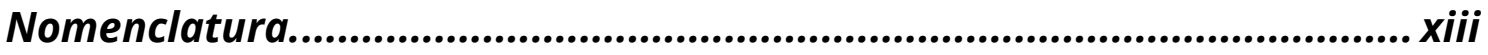

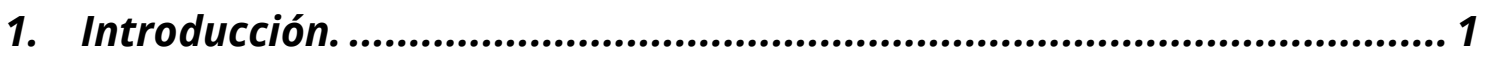

1.1 Ciclos de potencia para almacenamiento de $\mathrm{CO}_{2} \ldots \ldots \ldots \ldots \ldots . . . . . . . . . . . . . .11$

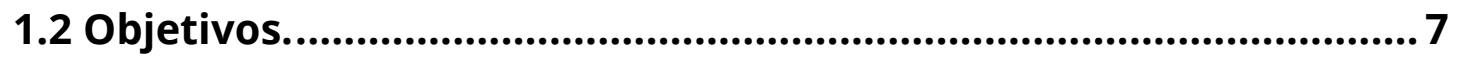

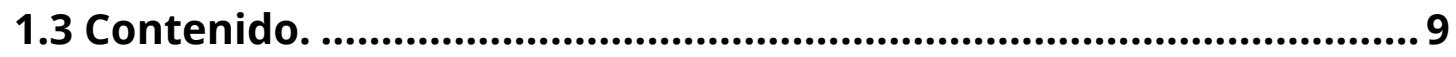

2. Estudio del punto de diseño del ciclo..................................................... 11

2.1 Modelo de simulación. .................................................................. 12

2.2 Obtención de parámetros característicos........................................ 16

2.3 Balance de gastos másicos y composición del gas recirculado.... 23

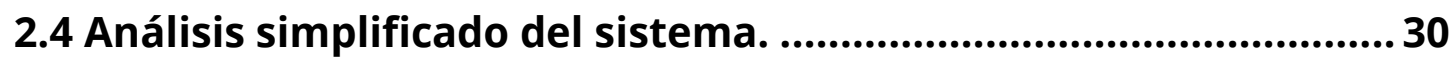

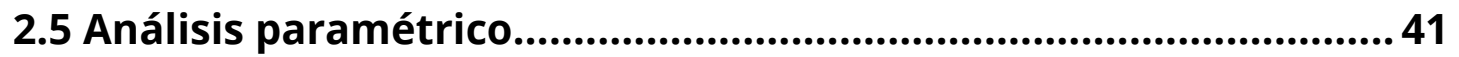

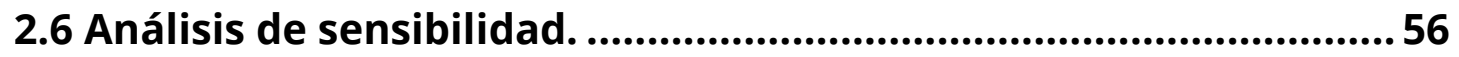

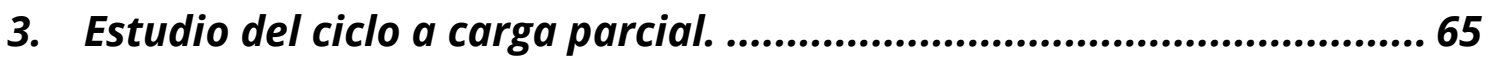

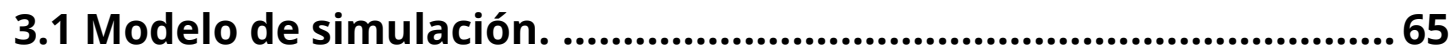

3.2 Obtención de los mapas de compresor y turbinas...........................68

3.3 Leyes de control para turbinas aeroderivadas.............................. 75

3.4 Leyes de control para turbinas monoeje.......................................... 78 
3.5 Ley de control mediante presión base.

3.6 Comparativa de leyes de control.................................................83 83

4. Estudio de la cámara de combustión............................................ 89

4.1 Modelo de simulación. ...............................................................90 90

4.2 Modelo auxiliar. .................................................................. 95

4.3 Comportamiento de la zona primaria. ...................................... 98

4.4 Comportamiento de la zona secundaria. ................................. 101

4.5 Comportamiento de la zona de dilución...................................... 105

4.6 Cámaras de combustión convencionales y del CCSC.................. 109

4.7 Sensibilidad ante cambios en el punto de diseño del ciclo......... 114

5. Conclusiones. ........................................................................... 117

Bibliografía. ......................................................................... 127 


\section{AGRADECIMIENTOS.}

En primer lugar quiero agradecer al profesor Gregorio Corchero que confiase en mí para realizar los trabajos que culminan en esta tesis, y que me haya guiado por el mundo del análisis de las actuaciones de ciclos termodinámicos. También quiero agradecer las aportaciones y el apoyo del profesor José Luis Montañés, y que haya accedido a codirigir la tesis. Muchas personas del departamento, tanto docentes como no docentes, me han ayudado cuando lo he necesitado y también se lo agradezco. Por supuesto, debo agradecer a los miembros del tribunal que hayan accedido a evaluar este trabajo.

Aunque no hayan colaborado directamente en esta tesis, debo agradecer a mis padres que la hayan hecho posible gracias a su constante apoyo y su cariño. A Nuria tengo que agradecerle que me haya soportado durante todo este tiempo.

Parte de la investigación presentada en esta tesis ha sido financiada por el proyecto CENIT 2007-2010 OPENAER, "Nuevas configuraciones de avión y motor para el futuro sistema de transporte aéreo", liderado por Industria de Turbopropulsores S.A, en la actividad 1.3, "Ciclos de turbina industrial para cero emisiones de $\mathrm{CO}_{2}$ ", liderada por ENDESA Generación, S.A, y con la participación de la Universidad Politécnica de Madrid. 


\section{RESUMEN.}

Esta tesis se centra en el análisis preliminar de ciclos combinados semicerrados con captura de $\mathrm{CO}_{2}$ mediante oxicombustión de gas natural y un gas rico en $\mathrm{CO}_{2}$ como fluido de trabajo. Se pone especial énfasis en la turbina de gas, con el objetivo de identificar las diferencias con las de ciclos combinados convencionales, y las barreras que puedan existir para el desarrollo o adaptación de componentes.

En primer lugar se estudia el ciclo en su punto de diseño, usando un modelo de simulación basado en componentes, cuyos parámetros se fijan a partir de los datos disponibles en la literatura científica. El análisis paramétrico del ciclo proporciona un posible punto de diseño con una relación de presiones de $30 \mathrm{y}$ una temperatura máxima del ciclo de $1600 \mathrm{~K}$, con valores de eficiencia y potencia específica comparables a los de ciclos convencionales para un amplio rango de relaciones de presiones, aunque los sistemas de generación de oxidante y de captura y almacenamiento de $\mathrm{CO}_{2}$ imponen considerables penalizaciones.

En segundo lugar se analiza el comportamiento del ciclo a carga parcial, adaptando mapas de turbomaquinaria funcionando con aire. Leyes de control usadas en ciclos convencionales llevan a resultados similares, sin grandes distinciones entre configuración monoeje o bieje. También se estudia una ley basada en el control del balance másico del ciclo semicerrado, que proporciona un muy elevado rendimiento a carga parcial.

En tercer lugar, y como caso particular de adaptación de componentes, se estudia el comportamiento de la cámara de combustión con gas recirculado, usando una red de reactores químicos sencilla. Los resultados sugieren el uso de temperaturas de equilibrio en la zona primaria moderadas, cerca de $1850 \mathrm{~K}$, para obtener tamaños comparables a los de cámaras de combustión de turbinas de gas funcionando con aire. 


\section{ABSTRACT.}

This thesis is focused on the analysis at the preliminary level of semi-closed combined cycles with $\mathrm{CO}_{2}$ capture, using a high $\mathrm{CO}_{2}$ content working gas and oxy-fuel combustion of natural gas. Emphasis is put on the gas turbine, in order to identify the differences with those of conventional combined cycles, and to try to find the possible barriers in the development of its components or the conversion of standard ones.

Firstly, the design point of the cycle is studied, by means of component based modelling. The parameters of the model are taken from data available in the scientific literature. The parametric analysis of the cycle suggests a feasible design point with a maximum pressure ratio of 30 and a maximum cycle temperature of $1600 \mathrm{~K}$, with values of efficiency and specific power similar to those of conventional combined cycles. The oxidant generation system and the $\mathrm{CO}_{2}$ capture and sequestration system represent a significant part of the losses.

Secondly, the part-load behaviour of the cycle is studied, using turbomachinery standard maps adapted to the high $\mathrm{CO}_{2}$ content working fluid. Standard control laws give similar results, without great differences between single and two shaft configurations. An alternative control law changing the working gas inventory of the semi-closed cycle gives very high efficiencies at part load.

Thirdly, as a particular case of conversion of standard components, the oxy-fuel combustion chamber is studied using simplified chemical reactor networks. This study suggests the use of moderate primary zone equilibrium temperatures, around $1850 \mathrm{~K}$, to obtain sizes similar to those of combustion chambers of conventional gas turbines. 


\section{NOMENCLATURA.}

A Área de paso.

$c_{p} \quad$ Capacidad térmica específica a presión constante

D Diámetro de la turbomáquina, gas de dilución.

d Relación de dilución, gasto másico de gas de dilución entre gasto másico de oxidante.

$e_{p} \quad$ Rendimiento politrópico

F Combustible

f Relación combustible-oxidante

far Relación combustible-aire.

h $\quad$ Entalpía específica

$k \quad$ Conductividad térmica.

$K_{A S U} \quad$ Consumo de la ASU por unidad de gasto másico de oxidante.

$K_{C C S} \quad$ Consumo del CCS por unidad de gasto másico de gas capturado.

$k_{g s} \quad$ Constante de combustión estequiométrica para el gas seco, $\left(1+f_{\text {est }}\right)\left(1-w_{\text {est }}\right)$.

$K_{P Z} \quad$ Fracción másica de diluyente inyectada en la ZP.

$k_{w} \quad$ Constante de combustión estequiométrica para el agua, $\left(1+f_{\text {est }}\right) w_{\text {est }}$.

L Longitud

m Gasto másico, masa total dentro de un reactor.

M Número de Mach, velocidad del gas dividida por la velocidad del sonido. 


\begin{tabular}{|c|c|}
\hline$m_{C, W}$ & Gasto másico de agua condensada. \\
\hline$m_{i j}$ & Gasto másico entre las estaciones i y j \\
\hline$N$ & Velocidad de giro de la turbomáquina. \\
\hline$n$ & Estación del PFR. \\
\hline$n_{d}$ & Estación de inicio de la dilución. \\
\hline$O$ & Oxidante. \\
\hline$P$ & Productos de combustión, presión. \\
\hline$P_{i t}$ & Presión de remanso en la estación i. \\
\hline ppmv & Partes por millón en volumen. \\
\hline $\begin{array}{l}\text { ppmvd } \\
@ 15 \% \mathrm{O}_{2}\end{array}$ & Composición corregida al $15 \%$ de $\mathrm{O}_{2}$. \\
\hline $\operatorname{Pr}$ & Número de Prandtl, $c_{p} \mu / k$ \\
\hline$P_{S T D}$ & Presión estándar, 101325 kPa. \\
\hline$R$ & Gas recirculado, constante del gas. \\
\hline$r$ & Relación entre el gasto másico de gas recirculado y de la ASU. \\
\hline $\operatorname{Re}$ & Número de Reynolds, $\rho U L / \mu$ \\
\hline$T_{i t}$ & Temperatura de remanso en la estación i. \\
\hline$t_{\text {res }}$ & Tiempo de residencia en un reactor. \\
\hline$T_{S T D}$ & Temperatura estándar, $288.15 \mathrm{~K}$. \\
\hline$T_{v}$ & Temperatura a la entrada de la turbina de vapor. \\
\hline$T_{v, \max }$ & Temperatura máxima admisible a la entrada de la turbina de vapor \\
\hline$U$ & Velocidad del gas. \\
\hline$V$ & Volumen. \\
\hline
\end{tabular}



W Agua, potencia generada por el ciclo.
$w_{c} \quad$ Relación entre el gasto másico de agua condensada recirculado y de la ASU.
$W_{i j} \quad$ Potencia introducida o extraída entre las estaciones i y j
$w_{r} \quad$ Fracción másica de agua en el gas recirculado
$X \quad$ Fracción volumétrica.
$x_{\text {refr }} \quad$ Porcentaje de gasto másico del HPC usado para refrigerar la HPT

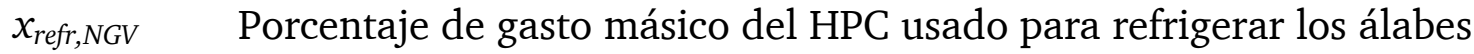 guía de la HPT

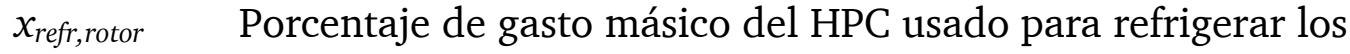 rotores de la HPT
Y $\quad$ Fracción másica.
z $\quad$ Relación entre el gasto másico capturado para almacenamiento y de la ASU.

\section{Acrónimos.}

AD Turbina aeroderivada (bieje).

ASU Unidad de separación de aire criogénica (Air Separation Unit).

BL Carga base (Base Load).

CC Cámara de combustión completa.

CCS Sistema de compresión para el almacenamiento de $\mathrm{CO}_{2}\left(\mathrm{CO}_{2}\right.$ Compression and Capture System).

CCSC Ciclo combinado semicerrado.

GS Gas seco obtenido al eliminar el agua de los productos de combustión.

HD Turbina monoeje (Heavy Duty).

HPC Compresor principal del ciclo de gas (High Pressure Compressor). 
HPT Turbina de alta presión del ciclo de gas (High Pressure Turbine).

HRSG Generador de vapor del ciclo combinado (Heat recovery Steam Generator).

LBO Apagado incipiente de la cámara de combustión (Lean Blowout)

LHV Poder calorífico inferior del combustible (Lower Heating Value)

LPC Compresor de baja presión del ciclo de gas (Low Pressure Compressor).

LPT Turbina de baja presión del ciclo de gas (Low Pressure Turbine).

NG Gas natural (Natural Gas).

NGV Álabes guía de la HPT (Nozzle Guide Vanes).

PFR Reactor tipo plug flow (Plug Flow Reactor).

PR Relación de presiones del ciclo de gas (Pressure Ratio).

PT Turbina de potencia del ciclo de gas (Power Turbine).

SFSA Sistema flexible de simulación de aerorreactores.

TET Temperatura a la entrada del rotor de la HPT (Turbine Entry Temperature).

UHC Hidrocarburos inquemados (Unburned Hydrocarbons).

VIGV Álabes guía de geometría variable del HPC.

VSV Estátores de geometría variable del HPC.

WSR Reactor de flujo continuo, bien agitado (Well Stirred Reactor).

ZD Zona de dilución de la cámara de combustión.

ZI Zona intermedia de la cámara de combustión.

ZP Zona primaria de la cámara de combustión.

ZS Zona secundaria de la cámara de combustión. 


\section{Símbolos griegos.}

$\gamma \quad$ Relación entre las capacidades térmicas específicas de un gas

$\delta_{i t} \quad$ Presión en la estación i dividida por la temperatura estándar.

$\Delta L_{\text {dilución }} \quad$ Longitud de la distribución de dilución adicional en la ZD.

$\Delta m \quad$ Gasto másico total de la distribución de dilución adicional en la ZD.

$\triangle T_{L B O} \quad$ Margen de estabilidad de la cámara de combustión.

$\Delta T_{\text {pinch }} \quad$ Mínima diferencia de temperatura entre el ciclo primario y el secundario en el HRSG.

$\triangle \eta_{A S U} \quad$ Disminución del rendimiento del ciclo debido a la ASU.

$\triangle \eta_{C C S} \quad$ Disminución del rendimiento del ciclo debido al CCS.

$\eta \quad$ Rendimiento isentrópico de turbomaquinaria, rendimiento del ciclo basado en LHV.

$\theta_{i j} \quad$ Relación de temperaturas totales entre las estaciones i y j

$\theta_{i t} \quad$ Temperatura en la estación i dividida por la temperatura estándar.

$\mu \quad$ Viscosidad dinámica del gas.

$\pi_{i j} \quad$ Relación de presiones totales entre las estaciones i y j

$\rho \quad$ Densidad del gas.

$\tau_{i j} \quad$ Potencia introducida o extraída entre las estaciones i y $\mathrm{j}$, adimensionalizada con $m_{C G} c_{p} T_{2 t}$

$\varphi \quad$ Relación de equivalencia del ciclo semicerrado

$\Phi \quad$ Relación de equivalencia real en la cámara de combustión.

$\omega \quad$ Potencia específica generada por el ciclo, relativa a $m_{C G}$.

$\omega_{i j} \quad$ Potencia específica introducida o retirada entre las estaciones i y $\mathrm{j}$, relativa a $m_{C G}$. 


\section{Estaciones del ciclo de gas.}

12 Oxidante a la salida de la ASU.

13 Salida del compresor del oxidante.

2 Entrada al HPC.

3 Salida del HPC.

3s Salida del HPC, asumiendo evolución isentrópica

31 Entrada a la ZP de la cámara de combustión.

4 Salida de la cámara de combustión

41 Entrada al estátor de la HPT, incluyendo el gasto de refrigeración.

43 Entrada al rotor de la HPT, incluyendo el gasto de refrigeración.

45 Salida de la HPT/entrada de la PT

$5 \quad$ Salida de la PT

5s Salida de la PT, asumiendo evolución isentrópica.

51 Salida del sobrecalentador del HRSG, entrada al evaporador.

52 Salida de evaporador del HRSG, entrada al economizador.

6 Salida del HRSG

\section{Estaciones del ciclo de vapor.}

a Salida del condensador/entrada a la bomba.

b Salida de la bomba.

c Salida del economizador del HRSG.

d Entrada al evaporador del HRSG

e Salida del evaporador del HRSG/entrada al sobrecalentador.

$f \quad$ Salida del sobrecalentador del HRSG/entrada a la turbina. 
$g \quad$ Salida de la turbina/entrada al condensador.

\section{Subíndices.}

CG Ciclo de gas.

CV Ciclo de vapor.

eq Condiciones de equilibrio.

est Relativo a la combustión estequiométrica.

ex Exceso respecto a la combustión estequiométrica.

out Salida del reactor

ref Condición de referencia para la ZP.

$t \quad$ Condiciones de remanso. 


\section{INTRODUCCIÓN.}

\subsection{Ciclos de potencia para almacenamiento de $\mathrm{CO}_{2}$.}

La creciente preocupación por el calentamiento global ha llevado al desarrollo de nuevos ciclos para la producción de potencia, diseñados para facilitar el proceso de captura y almacenamiento del dióxido de carbono generado.

Una de las técnicas bajo estudio se basa en la oxicombustión: una unidad de separación de aire criogénica, ASU (Air Separation Unit), extrae el nitrógeno del aire, obteniendo un flujo de oxígeno de alta pureza $(\geq 95 \%)$, que se usa para quemar el combustible. Los productos de combustión están compuestos en su mayor parte por $\mathrm{CO}_{2}$ y agua, siendo posible eliminar ésta mediante condensación y permitiendo que el flujo rico en $\mathrm{CO}_{2}$ resultante sea procesado para el almacenamiento. Dado que un proceso de oxicombustión pura llevaría a temperaturas muy elevadas, normalmente se recurre a un diluyente para reducirlas. Lo más sencillo es emplear los gases resultantes de la propia combustión, en distintas proporciones y con distintas configuraciones posibles para el ciclo.

La configuración más similar al ciclo combinado clásico utiliza un ciclo Brayton semicerrado, en el que se tiene un gas rico en $\mathrm{CO}_{2}$ como fluido de trabajo, en la cantidad necesaria para controlar el proceso de combustión y las condiciones de salida de la cámara. Tras la combustión se separa el $\mathrm{CO}_{2}$ del agua; la parte de $\mathrm{CO}_{2}$ generada durante el proceso se comprime para su almacenamiento y el resto se recircula. Dependiendo de la temperatura de salida del ciclo semicerrado se puede utilizar un ciclo secundario de recuperación como un ciclo Rankine con agua como fluido de trabajo, dando lugar a un ciclo combinado semicerrado, CCSC. 


\section{Introducción.}

Shao et al [1] fueron de los primeros en estudiar este tipo de ciclos. En su estudio utilizaron gas natural como combustible, y una posible opción para reducir el coste de generación de oxígeno en la ASU mediante su integración con el sistema de captura de $\mathrm{CO}_{2}$. Se presenta el rendimiento de un posible punto de diseño para un ciclo combinado, en el que la potencia de la turbina se ha estimado aplicando correcciones a la de una turbina convencional. El rendimiento del ciclo combinado pasa del 54\% del ciclo combinado convencional a un $45 \%$ al incluir el efecto de los sistemas de generación de oxígeno y compresión de $\mathrm{CO}_{2}$ para su captura. Aunque el concepto presentado resulta de interés, al no llevar a cabo un cálculo detallado de los ciclos no se puede tener en cuenta los efectos de variación de las variables de diseño del ciclo de gas, como la relación de presiones y la temperatura máxima, ni optimizar dicho ciclo.

Ulizar y Pilidis también estudiaron este tipo de ciclos hace casi dos décadas [2][4], empleando un gas sintético de bajo poder calorífico como combustible. En dicho estudio se realiza un análisis paramétrico de la respuesta de la eficiencia y rendimiento específico del ciclo combinado semicerrado a las variables de diseño de la turbina de gas. A partir de este estudio proponen como valores de diseño de la turbina de gas un valor de $1650 \mathrm{~K}$ para la temperatura a la entrada de la turbina de alta presión y un valor muy elevado, 60, para la relación de presiones de la turbina de gas. Este valor se escoge porque está cerca de dar el óptimo del rendimiento del ciclo combinado y porque la derivación de gas para la captura de $\mathrm{CO}_{2}$ se hace directamente de la salida del compresor, y se asume que este gas debe comprimirse hasta 60 bar, con lo que se elimina la necesidad de un sistema de compresión independiente. El rendimiento obtenido es relativamente bajo, 0.367, aunque estudiaron distintas alternativas para mejorar esta eficiencia. Aunque estos estudios tienen un indudable interés, no está claro que sus resultados se puedan aplicar a ciclos con un combustible convencional como el gas natural. 


\section{Introducción.}

Además de los ciclos basados en ciclos combinados convencionales, como los anteriores, se han investigado muchas otras alternativas, como el denominado MATIANT [5], que incluye un ciclo similar al Rankine con $\mathrm{CO}_{2}$ supercrítico como fluido de trabajo, sobre un ciclo Brayton regenerativo, también funcionando con $\mathrm{CO}_{2}$. Otro concepto consiste en el uso de agua como diluyente; la empresa Clean Energy Systems [6] tiene un prototipo de cámara de combustión de $20 \mathrm{MW}$, creada a partir de la de un motor cohete, en la que se quema el combustible con el oxígeno procedente de una ASU. La temperatura fin de combustión se controla mediante la inyección de agua en distintas estaciones a lo largo de dicha cámara. Con este generador de gas como base pueden crearse distintos ciclos como los de Martinez-Frias et al [7] y los del proyecto Zero Emissions Norwegian Plant (ZENG) [8]. Combinando en cierta forma los conceptos anteriormente presentados, los estudios de la Universidad de Tecnología de Graz [9]-[11] proponen un ciclo en el que se inyectan en la cámara de combustión tanto agua como dióxido de carbono recirculado, e incluyen estudios preliminares de los componentes. También son de interés otros sistemas que no utilizan una ASU criogénica para generar el oxígeno, como los métodos basados en membranas del AZEP [12], o el uso de un medio sólido para el transporte de oxígeno del método CLC [13].

Casi todos los estudios presentados anteriormente se centran en la obtención de la máxima eficiencia de un punto de funcionamiento determinado, con muy distintas consideraciones en cuanto a la composición de los gases y la eficiencia de los componentes. Kvamsdal et al [14] presentaron una interesante comparación desde el punto de vista de la eficiencia de 9 distintos conceptos de ciclos con combustión de gas natural y captura de $\mathrm{CO}_{2}$, incluyendo los ciclos con oxicombustión descritos, concluyendo que las considerables diferencias cualitativas entre ellos, como el distinto grado de madurez de las tecnologías implicadas, hacían muy difícil la selección. También es importante para la comparación considerar los costes asociados con las diversas tecnologías, como en el estudio de Davison y Thambimuthu [15]. 


\section{Introducción.}

Además de los estudios relacionados con la eficiencia de un punto del ciclo, normalmente el de plena carga, es necesario estudiar las actuaciones a carga parcial del ciclo, dado que el sistema deberá operar a carga parcial al menos durante las fases de arranque y parada, de modo que hay que asegurarse de que tanto el ciclo primario como el secundario pueden funcionar sin problemas. También hay que asegurarse del correcto funcionamiento en el caso de un cambio inesperado o una pérdida total de carga. Además, la estructura del sistema de producción de potencia puede requerir que el sistema opere a tiempo parcial durante períodos sustanciales de tiempo. Por lo tanto, las actuaciones del sistema en estas condiciones pueden tener un impacto considerable en el consumo total de combustible del ciclo.

La selección de la configuración de la turbina de gas del sistema está relacionada con estas consideraciones. Las turbinas de gas más grandes (del orden de 250 MW), usualmente denominadas "Heavy Duty", son máquinas monoeje, que operan a velocidad de rotación constante, determinada por la frecuencia de la red. Las máquinas multieje, de menor potencia, normalmente se desarrollan a partir del núcleo de una turbina de aviación, por lo que se las denomina aeroderivadas. Las actuaciones a carga parcial de las turbinas multieje suele ser mejor, gracias a que la velocidad del generador de gas puede variar para adaptarse a los cambios en las condiciones termodinámicas [16], [17]. Sin embargo, las turbinas monoeje pueden tener mejores actuaciones en diseño, gracias a las menores pérdidas en el colector de salida de la turbina [18]. Otra ventaja de las turbinas monoeje es que, al operar a velocidad de giro constante, no necesitan vencer la inercia asociada al cambiar la carga [17], [19], [20]. Además, el compresor puede actuar como freno en caso de una pérdida total de carga [19], [20].

La selección del patrón de control de potencia está ligada a la selección de la configuración de la turbina de gas. El método más sencillo consiste en la regulación del gasto másico de combustible. En turbinas monoeje, el uso de este 


\section{Introducción.}

control de forma exclusiva implicaría el cambio de la velocidad de giro con la carga, por lo que es necesario un control adicional si se quiere mantener la velocidad constante. Este método adicional suele consistir en el uso de álabes guía y estátores de geometría variable en el compresor [20]. En el caso de turbinas multieje, el grado de libertad adicional dado por el generador de gas permite el control mediante el gasto másico de combustible exclusivamente, pero también suelen estar equipadas con álabes guía y estatores de geometría variable en el compresor, para mejorar las actuaciones a carga parcial y el comportamiento del compresor ante las inestabilidades. Normalmente se usa un método mixto que proporciona una temperatura casi constante a la salida de la turbina, mejorando el rendimiento del ciclo combinado [21].

Algunos de estos aspectos en relación con sistemas CCSC han sido estudiados por Ulizar y Pilidis [2]-[4], para una turbina de dos ejes en el ciclo semicerrado, llegando a la conclusión de que la turbina no presenta retos más allá de lo usual en turbinas de gas convencionales, aunque hay que tener en cuenta que este ciclo tiene una muy alta relación de compresión y usa un combustible no convencional.

Un sistema similar al CCSC también ha sido estudiado por Imsland y Snarheim [22]-[25], usando una relación de presiones moderada (cerca de 20) y una temperatura de entrada a la turbina también moderada (1600 K), resultando en una temperatura de salida de la turbina de potencia muy alta (cerca de $1100 \mathrm{~K}$ ), mucho mayor que la utilizada en ciclos combinados convencionales. Sus estudios mostraron una influencia relativamente pequeña del método y la estrategia de control en las actuaciones a carga parcial, pero este resultado puede no ser de aplicación a temperaturas de salida de la turbina más bajas.

La cámara de combustión merece un apartado especial dentro de los componentes de los sistemas CCSC, dado que no se encuentran en la literatura métodos específicos para su diseño preliminar. En la oxicombustión diluida hay que tener en cuenta tres flujos distintos (el gas recirculado, el flujo de la ASU y 


\section{Introducción.}

el combustible). Por lo tanto, existe un parámetro adicional a considerar respecto al problema de la distribución del gasto másico en una turbina de gas estándar. A cambio aparece la restricción de que la combustión se produzca con un pequeño exceso de oxígeno, dado que este debe producirse en la ASU, con un consumo de potencia asociado. Tan et al [26] y Andersson y Johnsson [27] han estudiado la concentración necesaria de oxígeno para una combustión similar a la combustión con aire en quemadores semi-industriales, mientras que Ditaranto y Hals [28] han realizados estudios similares en una cámara de combustión con premezcla con una expansión súbita, y Kutne et al [29] y Nemitalla y Habib [30] han estudiado modelos de cámaras de combustión con flujo rotatorio. Sus resultados son similares, requiriendo aproximadamente un $30 \%$ de $\mathrm{O}_{2}$ para la combustión estable. Una complejidad añadida, no comentada por estos autores, es que cualquier exceso de oxígeno presente en los gases de combustión sería recirculado de nuevo hacia la cámara, de modo que la relación oxígenocombustible depende de la fracción de gas recirculado.

Además, el fluido de trabajo con una elevada concentración de $\mathrm{CO}_{2}$ es mucho más denso que el aire y que los gases de combustión con aire, lo que altera el gasto másico que puede pasar por un área determinada y el tiempo de residencia dentro de la cámara. La elevada concentración de $\mathrm{CO}_{2}$ también cambia los tiempos característicos asociados con la cinética química del monóxido de carbono y el nitrógeno. Como las propiedades termodinámicas y de transporte del $\mathrm{CO}_{2}$ también son diferentes de las del aire, las temperaturas, la distribución de gas adicional en la cámara, la estabilidad de combustión, y la longitud requerida para la combustión completa serán distintos de las de combustión con aire. Los resultados disponibles en la literatura son diversos, por ejemplo, Amato et al [31] han encontrado que la combustión diluida con $\mathrm{CO}_{2}$ requiere temperaturas mucho mayores que las de combustión con aire, para una cámara dada, mientras que Shroll et al [32] han encontrado que la transición entre los modos de estabilidad depende predominantemente de la temperatura de combustión adiabática de la mezcla, no de la composición. 
Un efecto adicional es que la mayor concentración de $\mathrm{CO}_{2}$ y $\mathrm{H}_{2} \mathrm{O}$ en los productos de combustión, cambia significativamente su emisividad respecto al caso de combustión con aire [27], [33]-[35]

\subsection{Objetivos.}

Esta tesis se va a centrar en el estudio a nivel preliminar de los ciclos combinados semicerrados, CCSC, con captura de $\mathrm{CO}_{2}$ mediante oxicombustión de gas natural y $\mathrm{CO}_{2}$ como diluyente principal, cuyo esquema se muestra en la Figura 1.

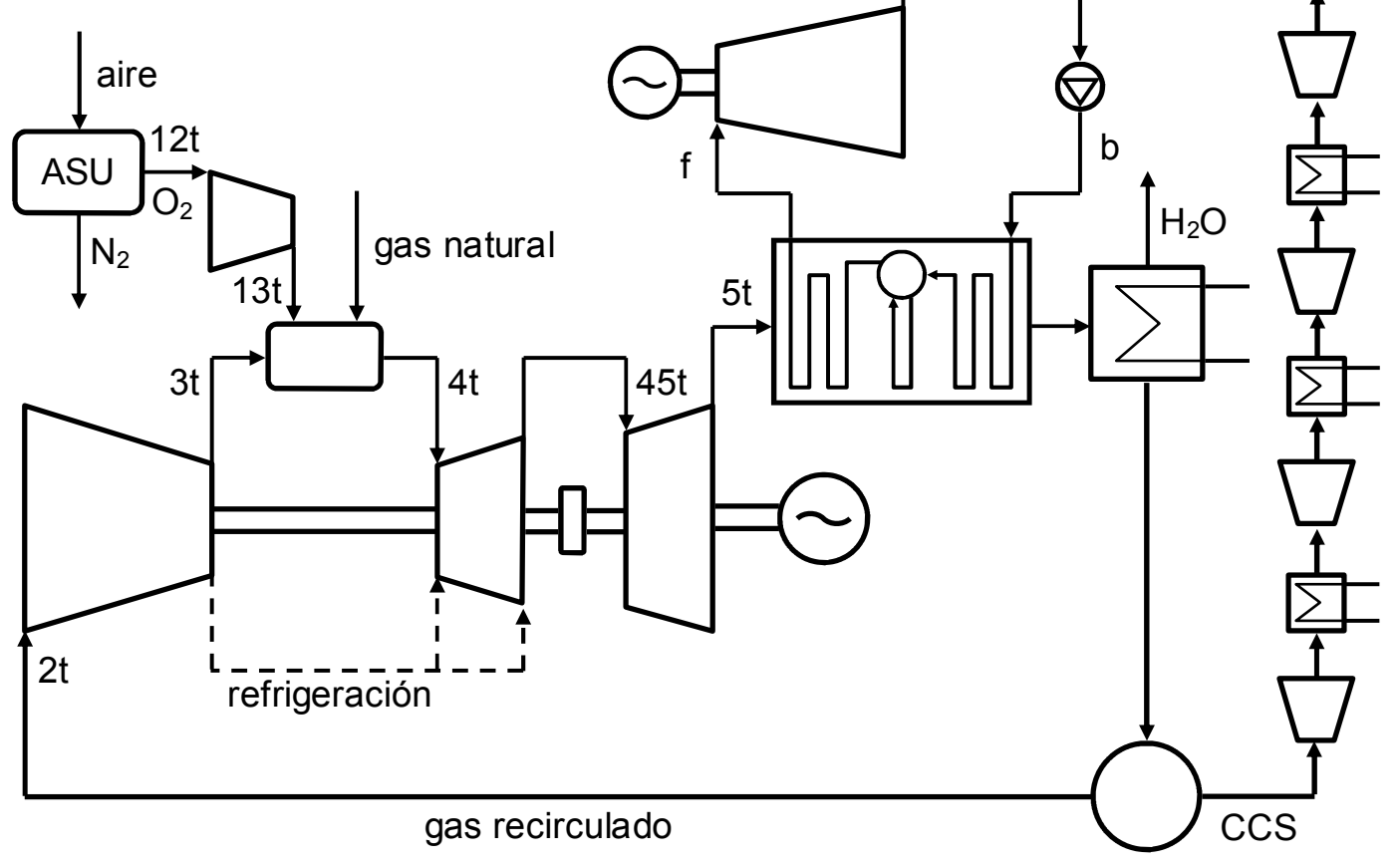

Figura 1. Esquema del ciclo combinado semicerrado objeto del estudio.

Esta configuración está basada en la de un ciclo combinado convencional, CC, en la que el ciclo primario es un ciclo Brayton semicerrado, cuyo fluido de trabajo es un gas rico en $\mathrm{CO}_{2}$, en la cantidad necesaria para controlar el proceso de combustión y las condiciones de salida de la cámara. El combustible es un gas natural, y el oxidante es un flujo de oxígeno de alta pureza $(\geq 95 \%)$, generado por la unidad de separación de aire criogénica, ASU (Air Separation Unit). Los 


\section{Introducción.}

productos de la combustión junto con el fluido de trabajo se pasan por una turbina de alta presión, HPT (High Pressure Turbine), que mueve el compresor del gas recirculado y los accesorios, incluyendo la ASU y el sistema de compresión para el almacenamiento de $\mathrm{CO}_{2}$, y una turbina de potencia, PT (Power Turbine), conectada a un generador eléctrico. Estos gases pasan después por un generador de vapor, HRSG (Heat Recovery Steam Generator), que transfiere parte de la energía disponible en el flujo al ciclo secundario, un ciclo Rankine con un solo nivel de presión, que utiliza agua como fluido de trabajo. Tras el paso de los gases por el HRSG, el agua generada por la combustión se separa mediante condensación. La parte de $\mathrm{CO}_{2}$ generada durante el proceso se deriva y se comprime para su almacenamiento en el sistema de compresión para el almacenamiento de $\mathrm{CO}_{2}$, $\mathrm{CCS}$, $\left(\mathrm{CO}_{2}\right.$ Compression and Capture System). Este subsistema consta de cuatro etapas, cada una de ellas formada por un compresor y un intercooler/condensador. El gas restante se recircula y se comprime de nuevo hasta las condiciones de entrada a la cámara de combustión.

A la vista de esta descripción, la parte principal del ciclo semicerrado puede consistir en una turbina de gas con uno o más ejes, similar a las turbinas de gas utilizadas para la generación de potencia salvo en que su fluido de trabajo es un gas rico en $\mathrm{CO}_{2}$. Por lo tanto, es natural pensar que una turbina de gas convencional o algunos de sus componentes podrían usarse para implementar este tipo de ciclos, llevando a cabo los cambios necesarios. Alternativamente, los métodos actuales de diseño de componentes se podrían adaptar para crear una turbina de gas adecuada para este tipo de ciclos.

El objetivo general de esta tesis es el análisis de las diferencias entre la turbina de gas del ciclo combinado semicerrado y las de ciclos combinados convencionales, para identificar las barreras que puedan existir a la hora del desarrollo/adaptación de componentes a nivel de diseño preliminar. Este es un paso previo necesario para el desarrollo detallado de la turbina de gas de este ciclo. Es importante considerar que el impacto de cualquier error en esta fase se 


\section{Introducción.}

vería amplificado en fases posteriores, por lo que hay que asegurarse de hacerlo correctamente, pese a su aparente simplicidad.

Este objetivo se va a dividir en tres objetivos relacionados:

- En primer lugar se pretende obtener la configuración del ciclo en su punto de diseño, mediante su modelado, optimización y posterior análisis, asumiendo tecnología actual en sus componentes y comparando su comportamiento con ciclos combinados convencionales.

- En segundo lugar se pretende obtener la configuración del ciclo fuera de su punto de diseño, incluyendo el número de ejes y las leyes de control. Para ello será necesario obtener un modelo apropiado de su comportamiento, incluyendo la estimación de los mapas de la turbomaquinaria.

- En tercer lugar, como caso particular de adaptación de componentes, se pretende evaluar el proceso de oxicombustión diluida y sus consecuencias en el diseño preliminar y el comportamiento de la cámara de combustión, en comparación con cámaras de combustión con aire.

\subsection{Contenido.}

En primer lugar se procederá a estudiar el ciclo en su punto de diseño. El sistema se modelará utilizando la metodología de simulación basada en componentes, usando la composición real de los gases con la suposición de composición de equilibrio en cada estación. Los parámetros necesarios para definir cada componente se obtendrán de los componentes de turbinas de gas existentes, obtenidos de la literatura científica. Sobre el modelo obtenido se realizará una optimización paramétrica de los parámetros fundamentales del ciclo de gas, la temperatura fin de combustión y la relación de presiones del ciclo, comparando su comportamiento con el de ciclos combinados convencionales. También se evaluarán otras alternativas para la mejora del ciclo, como el efecto del enfriamiento de los gases de entrada mediante un posible acoplamiento con la 


\section{Introducción.}

ASU. Una vez seleccionado un posible punto de diseño, se realizará un estudio de sensibilidad a la variación de los parámetros fundamentales y a algunos de los parámetros que definen los componentes.

El siguiente paso consistirá en la evaluación del comportamiento del sistema fuera de su punto de diseño. Para ello se construirá un modelo de simulación basado en componentes similar al usado para evaluar el punto de diseño. En este caso será necesario obtener mapas de comportamiento apropiados para la turbomaquinaria, mediante la adaptación de mapas de turbomaquinaria funcionando con aire. Mediante el estudio de este modelo se podrá seleccionar la arquitectura del ciclo de gas, que podrá constar de uno o de dos ejes, y una posible ley de control.

Como caso particular de adaptación de componentes, se va a estudiar el comportamiento de la cámara de combustión con gas recirculado. Para ello se va a construir un modelo simplificado de la cámara usando redes de reactores químicos, consistente en un reactor de flujo continuo, bien agitado, al que después se ha acoplado un reactor tipo Plug Flow. Después se analizará de forma general el comportamiento de este modelo, para seleccionar los parámetros más apropiados a usar para el diseño preliminar de la cámara del CCSC. De este modo se construirán modelos similares para el punto de diseño del CCSC y un ciclo convencional funcionando con aire, que podrán compararse para evaluar las diferencias y las posibles dificultades de la cámara de combustión del CCSC. También se realizará un análisis de sensibilidad del diseño de la cámara de combustión a la variación de los parámetros que definen el ciclo.

La tesis finalizará con una recapitulación de los resultados obtenidos y las dificultades previstas en el desarrollo del CCSC. 


\section{ESTUDIO DEL PUNTO DE DISEÑO DEL CICLO.}

En esta sección se pretende obtener la configuración del ciclo en su punto de diseño, mediante un modelo de simulación basada en componentes. En este tipo de modelos se divide el motor completo en componentes individuales, que se modelan por separado junto con un método de control que los relaciona. Aunque para cada componente pueden usarse modelos con distinto grado de fidelidad, para el nivel de diseño preliminar buscado en este estudio se usarán modelos cero dimensionales, en los que solo se calculan las propiedades del fluido de trabajo en las estaciones de entrada y salida de cada componente.

Aunque aparentemente muy simplificada, esta aproximación permite el análisis rápido de múltiples configuraciones de ciclos, requiriendo la especificación de una cantidad mínima de parámetros para definir cada componente. El análisis de este modelo permitirá la selección de los parámetros fundamentales que definen el ciclo combinado, con lo que se podrá realizar análisis más detallados para cada componente, hasta llegar al diseño final.

Los parámetros necesarios para definir cada componente se obtendrán mediante el estudio de los parámetros de componentes de turbinas de gas convencionales disponibles en la literatura científica, mediante simulaciones de turbinas convencionales para las que se tienen resultados de actuaciones y mediante el estudio de los parámetros usados en la literatura científica para ciclos semicerrados similares al objeto de estudio de esta tesis.

Sobre el modelo obtenido se realizará una optimización paramétrica de los parámetros fundamentales del ciclo de gas, la temperatura fin de combustión y la relación de presiones del ciclo, comparando su comportamiento con el de ciclos combinados convencionales. Mediante este proceso se seleccionará un posible punto de diseño que servirá como punto de partida para el análisis del 
comportamiento a carga parcial y el análisis más detallado de los componentes. Como el modelo utilizado y los parámetros son necesariamente aproximados, también se llevará a cabo un análisis de sensibilidad a los posibles cambios en estos parámetros.

\subsection{Modelo de simulación.}

Para la simulación del sistema se ha empleado el método de simulación basada en componentes, consistente en dividir el sistema en sus componentes. Estos son modelados por separado, siendo posible variar el grado de detalle dependiendo de las características que se consideren relevantes en cada caso. En este estudio se usarán modelos cero dimensionales, en los que solo se calculan las propiedades del fluido de trabajo en las estaciones de entrada y salida de cada componente.

Los cálculos se han realizado mediante un código propio del departamento de Mecánica de Fluidos y Propulsión Aeroespacial de la ETSIA, denominado Sistema Flexible de Simulación de Aerorreactores, SFSA. Este código ha sido verificado en diferentes configuraciones de sistemas de propulsión aérea y con otro código de cálculo de actuaciones del mismo departamento, Calact. Tanto el método de simulación, basado en componentes, como los de los componentes específicos usados en sistemas de propulsión aérea, son también aplicables a ciclos Rankine; la diferencia fundamental es el cambio de fluido de trabajo.

El ciclo de gas consta de:

- Unidad de separación de aire, ASU (Air Separation Unit). Es la encargada de suministrar el oxígeno necesario en el proceso de combustión del ciclo semicerrado. La ASU se modela como un componente externo al sistema en estudio; sus condiciones de alimentación, tanto de presión como de temperatura, no se ven afectadas por las condiciones de funcionamiento de la turbina de gas. En el ciclo con aire, este se obtiene del ambiente, representado por un componente específico. 
- Fase de compresión. En el ciclo semicerrado se utiliza un compresor encargado de comprimir el $\mathrm{O}_{2}$ producido por la ASU hasta las condiciones de suministro a la cámara de combustión, y un compresor principal, HPC (High Pressure Compressor), encargado de comprimir el gas recirculado hasta la presión deseada. La relación de compresión del HPC, PR (Pressure Ratio), es un parámetro fundamental por su influencia sobre las actuaciones del ciclo. Otro parámetro utilizado para caracterizar el HPC es su rendimiento isentrópico, $\eta_{H P C}$. El ciclo con aire se modela de forma similar salvo porque no requiere el compresor de $\mathrm{O}_{2}$.

- Fase de combustión en la cámara. Se supondrá combustión adiabática, y que el combustible, gas natural, es suministrado por la línea externa a las condiciones de presión necesarias para su inyección en la cámara. La temperatura a la salida de la cámara de combustión, $T_{4 t}$, es otro parámetro fundamental en las actuaciones del ciclo combinado,

- Fase de expansión. Constará de la turbina de alta, HPT (High Pressure Turbine), encargada de suministrar la potencia necesaria para la fase de compresión, y la turbina libre o turbina de potencia, PT (Power Turbine), encargada de suministrar potencia para uso externo, como puede ser un generador de energía eléctrica. El comportamiento de ambas turbinas se fijará mediante sus rendimientos isentrópicos, $\eta_{\text {HPT, }}$ у $\eta_{P T}$. Se supone que la HPT necesitará refrigeración para los álabes guía, NGV (Nozzle Guide Vanes), y el rotor, con un gas extraído de la salida del HPC. Los gastos másicos se fijarán mediante los respectivos porcentajes del gasto másico del compresor, $x_{\text {refr,NGV }}$ y $x_{\text {ref,rotor }}$. En el modelo de cálculo, se supone que el mezclado del gasto de refrigeración de los alabes guía con la corriente principal tiene lugar a presión constante, antes de la entrada al rotor, contribuyendo a la potencia de la turbina, mientras que el mezclado del gasto de refrigeración del rotor tiene lugar a presión constante, a la salida del rotor de la turbina. Esta es una práctica habitual en propulsión aérea cuando no se conocen los detalles del sistema de refrigeración. 
- Generador de vapor, HRSG (Heat recovery Steam Generator). Es responsable de la extracción del calor de los gases de escape para su uso por la turbina de vapor y, en consecuencia, de la adaptación parcial de los gases de escape a las condiciones de recirculado.

- Sistema de condensación de agua. Se encarga de enfriar los gases de escape del HRSG para la condensación del agua, la separación de dicha agua condensada, y la adecuación final a las condiciones de recirculado.

- Separación del gas recirculado. Una vez el agua ha sido extraída por condensación, los gases resultantes son separados en dos corrientes, una hacia el compresor principal de la turbina de gas para su recirculado y otra hacia el sistema de almacenamiento de $\mathrm{CO}_{2}$.

- Sistema de compresión para el almacenamiento de $\mathrm{CO}_{2}, \mathrm{CCS},\left(\mathrm{CO}_{2}\right.$ Compression and Capture System). Este subsistema consta de cuatro etapas, cada una de ellas formada por un compresor $y$ un intercooler/condensador, para reducir la potencia requerida para la compresión y eliminar el agua presente en los gases recirculados.

- Dependiendo de los casos puede existir un último cambiador de calor responsable de bajar la temperatura de los gases hasta la condiciones de recirculación.

El ciclo de vapor permite obtener un mayor rendimiento que el que se puede obtener del ciclo de gas por sí solo. Como se verá más adelante, el punto óptimo de funcionamiento de la turbina de gas como sistema independiente es completamente distinto al punto de funcionamiento como parte de un sistema de ciclo combinado. En cualquier caso, teniendo en cuenta que el objetivo principal es seleccionar el punto de diseño de la turbina de gas como componente del ciclo combinado, y que la utilización de varios niveles de presión en la turbina de vapor no afectará seriamente el punto de diseño de la turbina de gas, se ha implementado un ciclo Rankine con un solo nivel de presión. La implementación de tres niveles de presión originaría una ganancia en el rendimiento de la turbina de vapor del orden del 4-5\% [36]. 
El ciclo de vapor consta de:

- Fase de compresión. Formada por la bomba encargada de subir la presión del agua hasta las condiciones deseadas de entrada al HRSG. Su comportamiento vendrá dado por su rendimiento isentrópico.

- Generador de vapor. El HRSG es responsable de la extracción del calor de los gases de escape del ciclo semicerrado para su uso por la turbina de vapor. Para su simulación se ha modelado como un sistema constituido por un economizador, un evaporador, y un sobrecalentador. La temperatura de evaporación se fija mediante la presión de evaporación. El calor transferido entre ambos ciclos suele fijarse mediante el acercamiento o diferencia de temperatura entre ambos en dos puntos característicos. El primero es el acercamiento entre la temperatura a la salida de la turbina de gas y la temperatura de entrada a la turbina de vapor y el segundo es el acercamiento mínimo entre los ciclos, denominado usualmente "pinch point", que se suele producir en la estación a la entrada del evaporador desde el punto de vista del ciclo Rankine. Finalmente, también se especifica la aproximación a la saturación a la salida del economizador, variable que puede tener importancia a carga parcial.

- Fase de expansión. Formada por una turbina, encargada de suministrar la potencia necesaria para la fase de compresión, bomba, y proporcionar la potencia al exterior, cuyo comportamiento viene dado por su rendimiento isentrópico.

- Condensador. Es responsable del proceso de condensación para dejar el agua del ciclo Rankine en las condiciones de entrada a la bomba. Su comportamiento en diseño se suele modelar fijando la presión de condensación, lo que fija a su vez la temperatura de condensación.

El cálculo de las variables termodinámicas para el ciclo de gas se llevará a cabo suponiendo que en cada sección de interés la composición coincide con la que se 
obtendría en el caso de existir composición de equilibrio. Para su cálculo se ha utilizado un código basado en la minimización de la energía libre de Gibbs, y que se encarga de generar las tablas termodinámicas de interés para cada componente. Dicho código ha sido verificado con los códigos CET/CEA de la NASA, basados en la misma metodología y ampliamente aceptados en la comunidad técnica y científica [37].

El cálculo de las propiedades del agua para el ciclo Rankine se va a realizar utilizando a la formulación industrial de la International Association for the Properties of Water and Steam, versión 1997 (IAPWS-IF97) [38]. Este estándar ha sido adoptado por la American Society of Mechanical Engineers (ASME) y siendo un método cómodo y de gran velocidad de cálculo.

\subsection{Obtención de parámetros característicos.}

Las condiciones ambiente empleadas se muestran en la Tabla 1, considerándose como las condiciones ambiente de un emplazamiento típico. Se va a emplear una sola condición debido a que el propósito primario del estudio es la comparación con el funcionamiento con aire, pero podría ser interesante estudiar también la variación del comportamiento del diseño con distintas condiciones ambiente.

\begin{tabular}{cc}
\hline Temperatura $\left({ }^{\circ} \mathrm{C}\right)$ & 12 \\
Temperatura $(\mathrm{K})$ & 285 \\
Presión $(\mathrm{kPa})$ & 101.325 \\
\hline
\end{tabular}

Tabla 1. Condiciones ambiente.

Como ya se ha mencionado, la ASU no se modela de manera detallada, sino como un subsistema independiente del CCSC. Su consumo energético tiene una influencia muy importante en las actuaciones del sistema completo, y se modela como un consumo por unidad de gasto másico de oxidante generado. Según el informe especial sobre captura de $\mathrm{CO}_{2}$ del panel intergubernamental para el cambio climático [39], el consumo necesario para obtener $\mathrm{O}_{2}$ con una pureza del 95\% a $0.17 \mathrm{MPa}$ es de 200 a $240 \mathrm{kWh} / \mathrm{tO}_{2}$. En este estudio se va a utilizar un 
valor de $210 \mathrm{kWh} / \mathrm{tO}_{2}$, equivalente a $0.756 \mathrm{MJ} / \mathrm{kg}$, para un oxidante con un $95 \%$ de oxígeno y un 5\% de argón, en masa. Estos datos se resumen en la Tabla 2.

El ciclo semicerrado utiliza un gas de trabajo que se mezcla con el oxidante y el combustible. La composición de este gas recirculado no es obvia, estando ligada al balance másico de las especies en el ciclo. Por ejemplo, podría decidirse el uso de dióxido de carbono puro. A la salida se tendrían los productos de combustión completa para la reacción estequiométrica, junto con la cantidad de $\mathrm{CO}_{2}$ adecuada para controlar la temperatura. En este caso sería necesario eliminar toda el agua producida durante la combustión, reduciendo la temperatura hasta que condense, e introducir un proceso adicional que eliminase el resto de las especies producidas. El caso opuesto se obtendría empleando como gas recirculado los productos de combustión, de modo que para separar el gasto másico introducido en el ciclo semicerrado bastaría con dividir el flujo total a la salida.

\begin{tabular}{cc}
\hline Ar (\% masa) & 5 \\
$\mathrm{O}_{2}(\%$ masa $)$ & 95 \\
Masa molecular $(\mathrm{g} / \mathrm{mol})$ & 31.84 \\
Temperatura $\left({ }^{\circ} \mathrm{C}\right)$ & 18 \\
Temperatura $(\mathrm{K})$ & 291 \\
Presión $(\mathrm{kPa})$ & 125 \\
Consumo $\left(\mathrm{kWh} / \mathrm{tO}_{2}\right)$ & 210 \\
Consumo $(\mathrm{MJ} / \mathrm{kg})$ & 0.756 \\
\hline
\end{tabular}

Tabla 2. Propiedades de la ASU, incluyendo composición y propiedades del oxidante.

En este estudio se ha optado por un caso intermedio, en el que solo se separa parte del agua contenida en el gas producto de la combustión, mediante su condensación hasta una temperatura dada, dividiendo el flujo restante y recirculando la cantidad apropiada. Se ha tomado como temperatura de condensación la temperatura ambiente más $25^{\circ} \mathrm{C}$, y una presión del recirculado de $100 \mathrm{kPa}$. La manera de obtener la composición del recirculado para cada condición de funcionamiento se va a mostrar con más detalle en un apartado 
posterior, pero una composición representativa se muestra en la Tabla 3. La fracción másica de agua en este gas dependerá sobre todo de la presión y temperatura de condensación, cuando ambas se mantengan constantes se usará el valor de la tabla, un $2.8 \%$ en masa.

\begin{tabular}{cc}
\hline CO2 (\% masa) & 87.42 \\
Ar (\% masa) & 6.50 \\
O2 (\% masa) & 2.50 \\
N2 (\% masa) & 0.78 \\
H2O (\% masa) & 2.80 \\
Temperatura $\left({ }^{\circ} \mathrm{C}\right)$ & 37 \\
Temperatura (K) & 310 \\
Presión $(\mathrm{kPa})$ & 100 \\
\hline
\end{tabular}

Tabla 3. Composición representativa y propiedades del gas recirculado.

La composición y propiedades del combustible a emplear, un gas natural, NG (Natural Gas), se muestran en la Tabla 4.

\begin{tabular}{cc}
\hline N2 (\% volumen) & 1.540 \\
CO2 (\% volumen) & 0.980 \\
CH4 (\% volumen) & 87.000 \\
C2H6 (\% volumen) & 9.000 \\
C3H8 (\% volumen) & 1.340 \\
iC4H10 (\% volumen) & 0.116 \\
nC4H10 (\% volumen) & 0.014 \\
iC5H12 (\% volumen) & 0.015 \\
Masa molecular (g/mol) & 18.150 \\
Poder calorífico inferior, LHV (kJ/kg) & 47100 \\
\hline
\end{tabular}

Tabla 4. Composición y propiedades del combustible.

Para obtener los parámetros de los componentes del ciclo semicerrado se han seguido tres caminos: 
- Búsqueda de datos en la literatura abierta sobre componentes de turbinas de producción de potencia actualmente en funcionamiento y que proporcionó los datos de la Tabla 5.

- Simulación de turbinas existentes en el mercado de las que se tienen resultados de actuaciones globales, con el objetivo de encontrar datos adicionales, y cuyos resultados se muestran en la Tabla 6.

- Búsqueda de datos utilizados en ciclos similares al sistema objeto de esta tesis, que se han resumido en la Tabla 7.

\begin{tabular}{|c|c|c|c|c|c|c|c|c|c|c|c|c|c|c|}
\hline \multirow[b]{2}{*}{ Turbina de gas } & \multirow[b]{2}{*}{ PR } & \multirow[b]{2}{*}{$\begin{array}{l}\mathrm{T}_{4 \mathrm{t}} \\
(\mathrm{K})\end{array}$} & \multirow[b]{2}{*}{$\begin{array}{l}\text { TET } \\
(\mathrm{K})\end{array}$} & \multicolumn{2}{|c|}{ LPC } & \multicolumn{2}{|c|}{ HPC } & \multicolumn{2}{|c|}{ HPT } & \multicolumn{2}{|c|}{ LPT } & \multicolumn{2}{|c|}{$\mathrm{PT}$} & \multirow[b]{2}{*}{$\begin{array}{l}\mathrm{X}_{\mathrm{ref}} \\
(\%\end{array}$} \\
\hline & & & & $e_{p}$ & $\eta$ & $e_{p}$ & $\eta$ & $e_{p}$ & $\eta$ & $e_{p}$ & $\eta$ & $e_{p}$ & $\eta$ & \\
\hline Estado del arte 1997 [2] & - & - & 1473 & 90 & - & 89 & - & 87 & - & 87 & - & 87 & - & - \\
\hline LM2500 [40] & 18.8 & - & - & & & 88.9 & $83.7^{*}$ & & & & & & & - \\
\hline LM2500+ [40] & 23.3 & - & - & & & 91 & $86.4^{*}$ & & & & & & & - \\
\hline $\begin{array}{l}\text { "Modern Reference } \\
\text { Engine" [41] }\end{array}$ & 17 & - & 1550 & & & 89.5 & $84.8^{*}$ & 91 & $93.6^{*}$ & & & & & 6 \\
\hline SGT5-4000F [42] & 17 & 1700 & - & & & 91.5 & $87.7^{*}$ & 89 & $92.1 *$ & & & & & 22 \\
\hline Idem, simulación [42] & 17 & 1700 & - & & & $91.7^{*}$ & 87.95 & 85.77 & 89.65 & & & & & 22 \\
\hline
\end{tabular}

Tabla 5. Datos de eficiencia de componentes y fracción másica de refrigeración obtenidos de la literatura abierta. * estimado mediante fórmulas de gas ideal: $\gamma=1.4$ compresores, $\gamma=1.33$ turbinas.

En estas tablas aparecen dos temperaturas características, $T_{4 t}$, que es la temperatura a la salida de la cámara de combustión y TET (Turbine Entry Temperature), que suele usarse para representar la temperatura a la entrada del rotor de la turbina de alta, a la salida del mezclado de la refrigeración de los NGVs con la corriente principal. Las $T_{4 t}$ están comprendidas entre $1600 \mathrm{~K}$ y 1700 $\mathrm{K}$, y se tomará $T_{4 t}=1600 \mathrm{~K}$ como representativa en el estudio paramétrico.

La relación de presiones presenta ciertas diferencias entre ciclo abierto y semicerrado. Mientras que las turbinas funcionando con aire rondan el valor 20, las de ciclo semicerrado están entre 20 y 40 . El efecto de este parámetro se analizará en detalle en el estudio paramétrico. 
2. Estudio del punto de diseño del ciclo.

Las fracciones másicas para refrigeración están comprendidas entre el 16\% y 23\%, no apreciándose una diferencia clara entre los valores empleados en ciclo abierto, o ciclo semicerrado. Como base para el estudio paramétrico se va utilizar un total de $20 \%$, con $13.33 \%$ para el NGV y el resto para el rotor.

\begin{tabular}{|c|c|c|c|c|c|c|c|c|c|c|c|c|c|c|}
\hline \multirow[b]{2}{*}{ Turbina } & \multirow[b]{2}{*}{$\mathrm{PR}$} & \multirow[b]{2}{*}{$\eta$} & \multirow[b]{2}{*}{$\begin{array}{l}\mathrm{T}_{4 \mathrm{t}} \\
(\mathrm{K}) \\
\end{array}$} & \multirow[b]{2}{*}{$\begin{array}{l}\text { TET } \\
(\mathrm{K}) \\
\end{array}$} & \multirow[b]{2}{*}{$\begin{array}{l}\mathrm{T}_{5 \mathrm{t}} \\
(\mathrm{K}) \\
\end{array}$} & \multicolumn{2}{|c|}{ HPC } & \multicolumn{2}{|c|}{ HPT } & \multicolumn{2}{|c|}{ PT } & \multicolumn{3}{|c|}{$\mathrm{x}_{\text {refr }}(\%)$} \\
\hline & & & & & & $e_{p}$ & $\eta$ & $e_{p}$ & $\eta$ & $e_{p}$ & $\eta$ & NGV & Rotor & total \\
\hline $\begin{array}{l}\text { MS7001FA } \\
60 \mathrm{~Hz} \text { [43] }\end{array}$ & 14.8 & 36.2 & - & 1561 & 867 & & & & & & & & & \\
\hline Idem SFSA & 14.8 & 36.6 & 1678 & 1562 & 878 & 90.1 & 86.0 & 86.5 & 88.3 & 85.4 & 87.9 & 12 & 9 & 21 \\
\hline error $(\%)$ & 0.0 & 1.2 & - & 0.0 & 1.3 & & & & & & & & & \\
\hline $\begin{array}{l}\text { MS6001C } \\
60 \mathrm{~Hz}[44]\end{array}$ & 19.6 & 36.5 & - & - & 854 & & & & & & & & & \\
\hline Idem SFSA & 19.6 & 36.6 & 1640 & 1555 & 856 & 89.5 & 84.7 & 83.0 & 85.6 & 82.5 & 85.5 & 10 & 6 & 16 \\
\hline error $(\%)$ & 0.0 & 0.3 & - & - & 0.1 & & & & & & & & & \\
\hline $\begin{array}{c}\text { M701F } \\
60 \mathrm{~Hz}[45]\end{array}$ & 16 & 36.6 & 1623 & $1548 *$ & 822 & & & & & & & & & \\
\hline Idem SFSA & 16 & 36.8 & 1606 & 1497 & 835 & 90.6 & 86.5 & 85.4 & 87.5 & 85.0 & 87.5 & 12.3 & 8.7 & 21 \\
\hline error (\%) & 0.0 & 0.5 & -1.0 & -3.3 & 1.5 & & & & & & & & & \\
\hline
\end{tabular}

Tabla 6. Resultados de la simulación de turbinas existentes mediante SFSA, ajustando a los datos obtenidos de la literatura * estimado como $T_{4 t}-75 \mathrm{~K}$.

\begin{tabular}{|c|c|c|c|c|c|c|c|c|c|c|c|c|c|c|}
\hline \multirow[b]{2}{*}{ Turbina } & \multirow[b]{2}{*}{$\mathrm{PR}$} & \multirow[b]{2}{*}{$\eta$} & \multirow[b]{2}{*}{$\begin{array}{l}\mathrm{T}_{4 \mathrm{t}} \\
(\mathrm{K})\end{array}$} & \multirow[b]{2}{*}{$\begin{array}{r}\text { TET } \\
(\mathrm{K})\end{array}$} & \multirow[b]{2}{*}{$\begin{array}{l}\mathrm{T}_{5 \mathrm{t}} \\
(\mathrm{K})\end{array}$} & \multicolumn{2}{|c|}{ HPC } & \multicolumn{2}{|c|}{ HPT } & \multicolumn{2}{|c|}{$\mathrm{PT}$} & \multicolumn{3}{|c|}{$\mathrm{x}_{\text {refr }}(\%)$} \\
\hline & & & & & & $e_{p}$ & $\eta$ & $\mathrm{e}_{\mathrm{p}}$ & $\eta$ & $\mathrm{e}_{\mathrm{p}}$ & $\eta$ & NGV & Rotor & total \\
\hline SCOC-CC & & & & & & & & & & & & & & \\
\hline $\begin{array}{c}\text { ENCAP } \\
{[46]}\end{array}$ & 40.1 & $40.1 *$ & 1698 & 1504 & 902 & 91.5 & 88.4 & 86.4 & 89.7 & - & - & 23.3 & -- & 23.3 \\
\hline $\begin{array}{c}\text { SCOC-CC } \\
{[14]}\end{array}$ & 30 & - & 1601 & - & 943 & 91 & - & 91 & - & - & - & - & - & - \\
\hline $\begin{array}{l}\text { SC-CC, } \\
\text { SFSA } 20\end{array}$ & 20 & 32.0 & 1600 & 1489 & 955 & 90.2 & 87.0 & 0.878 & 89.0 & 87.2 & 89.0 & 13.3 & 6.7 & 20 \\
\hline $\begin{array}{l}\text { SC-CC, } \\
\text { SFSA } 30\end{array}$ & 30 & 34.7 & 1600 & 1494 & 905 & 90.5 & 87.0 & 0.875 & 89.0 & 87.0 & 89.0 & 13.3 & 6.7 & 20 \\
\hline $\begin{array}{l}\text { SC-CC, } \\
\text { SFSA } 40\end{array}$ & 40 & 36.3 & 1600 & 1497 & 872 & 90.7 & 87.0 & 0.873 & 89.0 & 86.9 & 89.0 & 13.3 & 6.7 & 20 \\
\hline
\end{tabular}

Tabla 7. Comparación de los rendimientos empleados en la simulación del ciclo semicerrado con otros ciclos similares mencionados en la literatura. * calculado a partir de los datos del artículo.

Existe cierta dispersión en los valores de las eficiencias de la turbomaquinaria, se escogerán como valores representativos 0.87 para los compresores y 0.89 para las turbinas. Se va a asumir que las turbomáquinas funcionando con gas recirculado pueden diseñarse para obtener eficiencias similares a las de turbomáquinas funcionando con aire. 
Los valores característicos de los parámetros utilizadas en la simulación se han resumido en la Tabla 8, donde aparecen también los valores de las pérdidas utilizados habitualmente para otros componentes como el eje o la pérdida de presión en la cámara de combustión.

\begin{tabular}{cc}
\hline \multicolumn{2}{c}{ Valores base } \\
\hline Relación de presiones & 30 \\
$\mathrm{~T}_{4 \mathrm{t}}(\mathrm{K})$ & 1600 \\
\hline Rendimientos y pérdidas & 0.87 \\
\hline Rendimiento isentrópico compresor & 0.999 \\
Rendimiento cámara combustión & 3 \\
Pérdida de presión cámara de combustión (\%) & 0.89 \\
Rendimiento isentrópico turbina de alta & 0.89 \\
Rendimiento isentrópico turbina de baja & 0.99 \\
Rendimiento eje & 4 \\
\hline Pérdida de presión generador de vapor (\%) & 13.33 \\
\hline Estación sangrado: salida compresor & 6.67 \\
\hline Sangrado NGV (\%) \\
Sangrado Rotor (\%)
\end{tabular}

Tabla 8. Eficiencias de componentes de la turbina de gas.

El subsistema de compresión de $\mathrm{CO}_{2}$ (CCS) consta de cuatro etapas. Cada una de ellas está formada por un compresor y un intercooler/condensador para reducir el trabajo requerido para la compresión y eliminar el agua presente en los gases recirculados. Cada etapa deja los gases a $37{ }^{\circ} \mathrm{C}, 25{ }^{\circ} \mathrm{C}$ por encima de la temperatura ambiente. Los valores correspondientes a cada etapa y el consumo del subsistema completo se muestran en la Tabla 9.

Los parámetros de diseño del ciclo de vapor se van a fijar teniendo en cuenta que este ciclo no se pretende modelar con mucho detalle, sino para facilitar la comparación entre los ciclos combinados convencionales y CCSC. La temperatura a la salida del generador de vapor es un parámetro fundamental en la eficiencia del ciclo de vapor, y vendrá dada por el acoplamiento con el ciclo de 
gas, pero no debería superar la temperatura admisible a la entrada de la turbina, $T_{v, \max }$, que se va a tomar igual a $900 \mathrm{~K}$. En diseño se ha fijado imponiendo el acercamiento con la temperatura del ciclo de gas en dicho punto, con un valor igual a $25 \mathrm{~K}$. Si el valor obtenido superase $T_{v, \max }$ se haría igual a este.

\begin{tabular}{cc}
\hline Por etapa & \\
\hline Relación de compresión & 3.24 \\
Rendimiento isentrópico compresor & 0.85 \\
Pérdida de presión en el intercooler $(\%)$ & 3 \\
Temperatura salida intercooler $\left({ }^{\circ} \mathrm{C}\right)$ & 37 \\
\hline Temperatura salida intercooler $(\mathrm{K})$ & 310 \\
\hline Para el subsistema de 4 etapas \\
\hline Consumo $(\mathrm{MJ} / \mathrm{kg})$ & 0.36 \\
\hline
\end{tabular}

Tabla 9. Parámetros cada etapa del subsistema de compresión de $\mathrm{CO}_{2}$, para un subsistema de 4 etapas, y consumo total de potencia por unidad de gasto másico.

Los otros parámetros de diseño del ciclo del ciclo de vapor son la presión máxima y la presión del condensador, que fijan la temperatura de evaporación y de condensación del ciclo. La turbina y la bomba se van a simular imponiendo el rendimiento isentrópico, de manera similar a los componentes del ciclo de gas. Los valores utilizados se han resumido en la Tabla 10.

\begin{tabular}{cc}
\hline Presión máxima (MPa) & 10 \\
Rendimiento isentrópico bomba & 0.80 \\
Aproximación a la saturación entrada evaporador (K) & 10 \\
Acercamiento entrada evaporador (Pinch point) (K) & 5 \\
Acercamiento salida sobrecalentador (K) & 25 \\
Pérdida de presión generador de vapor (\%) & 4 \\
Temperatura máxima admisible en la turbina (K) & 900 \\
Rendimiento isentrópico turbina & 0.89 \\
Rendimiento del eje & 0.99 \\
Presión del condensador (kPa) & 5 \\
\hline
\end{tabular}

Tabla 10. Parámetros del ciclo de vapor. 


\subsection{Balance de gastos másicos y composición del gas recirculado.}

Para obtener la composición del gas recirculado se va a partir de un proceso hipotético de oxicombustión pura, en el que se tiene un gasto másico de oxidante extraído de la ASU, $m_{A S U}$, y un gasto másico de combustible $m_{F}$. La composición del combustible se expresará mediante el símbolo $F$.

El gasto másico de combustible, $m_{F}$, se suele referir al gasto másico de oxidante, $m_{A S U}$, mediante la variable

$$
f=\frac{m_{F}}{m_{A S U}}
$$

También es habitual utilizar el valor de $f$ correspondiente a la combustión estequiométrica, $f_{\text {est }}$, para crear una variable similar a la relación de equivalencia

$$
\varphi=\frac{f}{f_{\text {est }}}
$$

Asumiendo combustión con exceso de oxígeno $(\varphi<1)$, la cantidad de oxidante que interviene en la combustión es

$$
m_{A S U, e s t}=\varphi m_{A S U} \text {. }
$$

Utilizando estas definiciones, la combustión de $m_{A S U}$ y $m_{F}$ generará los productos de combustión estequiométrica con un gasto másico

$$
m_{P, e s t}=m_{F}+m_{A S U, \text { est }}=\left(1+f_{e s t}\right) \varphi m_{A S U}
$$

y con un exceso de oxidante

$$
m_{A S U, e x}=(1-\varphi) m_{A S U} .
$$

La composición de los productos de combustión estequiométrica, $P_{\text {est }}$, es función únicamente de la composición del oxidante y del combustible empleados. Se 
denominará gas seco, GS, a los productos de combustión estequiométrica menos el agua, $W$. La fracción másica de agua, $w_{\text {est }}$, se va a utilizar para definir esta separación, en la forma

$$
P_{\text {est }}=\left(1-w_{\text {est }}\right) G S+w_{\text {est }} W \text {. }
$$

A los productos de combustión estequiométrica y el exceso de oxidante hay que añadir el gas recirculado, $R$. La cantidad que hay que recircular vendrá dada por la temperatura final deseada, que está limitada por los materiales de la turbina y es un parámetro fundamental en los estudios de optimización del ciclo. El gasto másico de gas recirculado, $m_{R}$, también se va a expresar en relación con el gasto másico de la ASU, mediante el parámetro

$$
r=\frac{m_{R}}{m_{A S U}}
$$

Se va a asumir que la composición de gas recirculado se puede expresar como una combinación de gas seco, agua y oxidante, dada por las fracciones másicas correspondientes, $Y_{G S, R}, Y_{W, R}$ e $Y_{A S U, R}$.

$$
m_{R} R=r m_{A S U}\left(Y_{G S, R} G S+Y_{A S U, R} A S U+Y_{W, R} W\right)
$$

La fracción másica de agua del recirculado se considera conocida, con un valor $w_{r}$.

$$
Y_{W, R}=w_{r} .
$$

Si se denomina $P$ a los productos de combustión junto con el recirculado en la estación inmediatamente anterior a la condensación del agua, su gasto másico y composición pueden expresarse como

$$
m_{P} P=m_{P, e s t} P_{e s t}+m_{A S U, e x} A S U+m_{R} R,
$$

con un gasto másico total 


$$
m_{P}=(1+f+r) m_{A S U}
$$

y unas fracciones másicas, $Y_{G S, P}, Y_{W, P}$ e $Y_{A S U, R}$, que se pueden obtener de las correspondientes de los productos de combustión estequiométrica, exceso de oxidante y gas recirculado,

$$
(1+f+r)\left\{\begin{array}{c}
Y_{G S, P} \\
Y_{A S U, P} \\
Y_{W, P}
\end{array}\right\}=\left\{\begin{array}{c}
\varphi\left(1+f_{e s t}\right)\left(1-w_{e s t}\right)+r Y_{G S, R} \\
(1-\varphi)+r Y_{A S U, R} \\
\varphi\left(1+f_{e s t}\right) w_{e s t}+r w_{r}
\end{array}\right\}
$$

Estas expresiones pueden expresarse de forma abreviada, mediante la definición de los parámetros:

$$
\begin{gathered}
k_{g s}=\left(1+f_{\text {est }}\right)\left(1-w_{\text {est }}\right) \\
k_{w}=\left(1+f_{\text {est }}\right) w_{\text {est }}
\end{gathered}
$$

Como la composición tras la condensación de agua es igual a la del gas recirculado, se puede afirmar que la relación entre las fracciones másicas de GS y ASU no puede verse alterada por este proceso, obteniendo la relación

$$
\frac{Y_{A S U, R}}{Y_{G S, \mathrm{R}}}=\frac{Y_{A S U, P}}{Y_{G S, P}}=\frac{(1-\varphi)+r Y_{A S U, R}}{r Y_{G S, R}+\varphi k_{g s}},
$$

que se puede simplificar, obteniendo

$$
\frac{Y_{A S U, R}}{Y_{G S, R}}=\frac{1-\varphi}{\varphi k_{g S}}
$$

Y, teniendo en cuenta que las fracciones másicas deben sumar la unidad,

$$
Y_{A S U, R}=\frac{(1-\varphi)\left(1-w_{r}\right)}{1-\varphi+\varphi k_{g s}}
$$


Para completar el análisis del balance másico en el ciclo hay que obtener los gastos másicos de agua condensada y el gasto másico de gas que hay que almacenar.

Si se define el gasto másico de agua condensada como una fracción de $m_{A S U}$,

$$
m_{C, W}=w_{c} m_{A S U}
$$

Y se hace lo mismo con gasto másico de gas a extraer para su almacenamiento

$$
m_{C C S}=z m_{A S U} \text {. }
$$

Como el gasto másico total inyectado en el ciclo es

$$
m_{F}+m_{A S U}=\left(1+\varphi f_{e s t}\right) m_{A S U}
$$

la variable z puede expresarse en función de $w_{c}$,

$$
z=1+\varphi f_{\text {est }}-w_{c}
$$

Como este gas tiene la misma composición que el recirculado, la cantidad de agua extraída es

$$
m_{C C S, W}=z w_{r} m_{A S U}
$$

Como se ha visto con anterioridad, el gasto másico de agua antes de la condensación es

$$
\left(\varphi k_{w}+r w_{r}\right) m_{A S U}
$$

Con lo que el balance de gasto másico de agua en el ciclo puede expresarse en la forma

$$
\left(\varphi k_{w}+r w_{r}-w_{c}-z w_{r}\right) m_{A S U}=r w_{r} m_{A S U}
$$

De donde se obtiene 


$$
w_{c}=\frac{\varphi k_{w}-\left(1+\varphi f_{e s t}\right) w_{r}}{1-w_{r}}
$$

$\mathrm{y}$

$$
z=\frac{1+\varphi f_{e s t}-\varphi k_{w}}{1-w_{r}}
$$

Para la composición dada anteriormente para la ASU y el combustible se obtienen los valores de los parámetros de la Tabla 11.

$$
\begin{array}{cc}
\mathrm{f}_{\text {est }} & 0.253815 \\
\mathrm{w}_{\text {est }} & 0.41476 \\
\mathrm{k}_{\mathrm{gs}} & 0.73378 \\
\mathrm{k}_{\mathrm{w}} & 0.52003 \\
\hline
\end{array}
$$

Tabla 11. Parámetros del balance de masas del ciclo semicerrado.

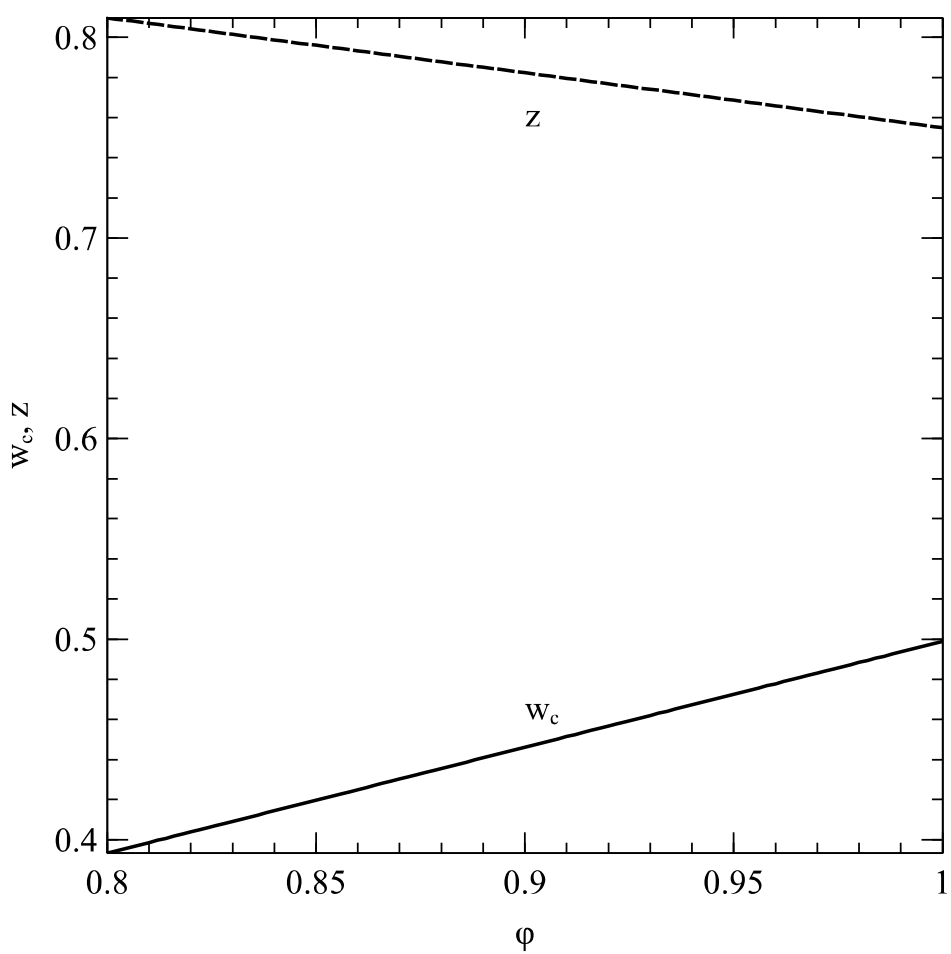

Figura 2. Variación con $\varphi$ de la fracción másica de agua condensada, $w_{c}$ y de gas a extraer, z, para la composición de la ASU y el combustible del CCSC. 
La forma en que los valores de $w_{c}$ y $z$ varían $\operatorname{con} \varphi$ se muestra en la Figura 2, mientras que los valores para $\varphi=1$ y $\varphi=0.98$, se muestran en la Tabla 12 .

\begin{tabular}{ccc}
\hline$\varphi$ & $\mathrm{w}_{\mathrm{c}}$ & $\mathrm{z}$ \\
\hline 0.98 & 0.4883 & 0.7604 \\
1.0 & 0.4989 & 0.7549 \\
\hline
\end{tabular}

Tabla 12. Valores de $w_{c} y z$ para $\varphi=1$ y $\varphi=0.98$.

Para mostrar los efectos esperados del uso de la composición correcta del gas recirculado se va a mostrar la variación con la temperatura de las propiedades de los gases de trabajo de una turbina convencional, es el aire a la entrada y los resultados de una combustión con $\mathrm{CH}_{4}$ y relación aire combustible igual a 0.02, que emulan los gases a la salida de la turbina de potencia, denominado aire $5 \mathrm{t}, \mathrm{y}$ los gases de trabajo de un CCSC con $P R=30$ y $T_{4 t}=1600 \mathrm{~K}$, tanto el gas recirculado a la entrada del ciclo semicerrado, denominado recirculado, como el de salida de la turbina de potencia, denominado recirculado 5t. También se ha va incluir el $\mathrm{CO}_{2}$ puro, para comparar con la composición más simplificada posible. La composición de estos gases se muestra en la Tabla 13.

\begin{tabular}{cccccc}
\hline gas & $\begin{array}{c}\mathrm{CO}_{2} \\
\% \text { masa }\end{array}$ & $\begin{array}{c}\mathrm{O}_{2} \\
\% \text { masa }\end{array}$ & $\begin{array}{c}\mathrm{H}_{2} \mathrm{O} \\
\% \text { masa }\end{array}$ & $\begin{array}{c}\mathrm{Ar} \\
\% \text { masa }\end{array}$ & $\begin{array}{c}\mathrm{N}_{2} \\
\% \text { masa }\end{array}$ \\
\hline aire & 0.05 & 23.14 & 0.00 & 1.29 & 75.52 \\
aire 5t & 6.12 & 13.93 & 5.27 & 1.26 & 73.43 \\
recirculado & 87.42 & 2.50 & 2.80 & 6.50 & 0.78 \\
recirculado 5t & 83.75 & 2.39 & 6.88 & 6.23 & 0.75 \\
$\mathrm{CO}_{2}$ & 100.00 & 0.00 & 0.00 & 0.00 & 0.00 \\
\hline
\end{tabular}

Tabla 13. Composición del gas recirculado para CCSC con $P R=30, T_{4 t}=1600$, gas recirculado $5 t$ composición a la salida de la turbina de gas, aire 5t posible composición a la salida de una turbina de gas convencional para combustión con $\mathrm{CH}_{4}$, relación combustible aire 0.02 .

La capacidad térmica específica a presión constante, $c_{p}$, para la composición de equilibrio de cada gas a la temperatura correspondiente, se muestra en la Figura 3. Un rasgo muy importante de esta propiedad es la considerable variación con la temperatura de los gases ricos en $\mathrm{CO}_{2}$, que comienzan en un valor muy inferior al del aire y terminan en un valor muy superior, incluso al de los productos de combustión con aire, aire 5 t. Como se verá más adelante, la 
considerable variación de esta propiedad con la temperatura para el gas recirculado 5t hace que cambie el comportamiento cualitativo del generador de vapor.

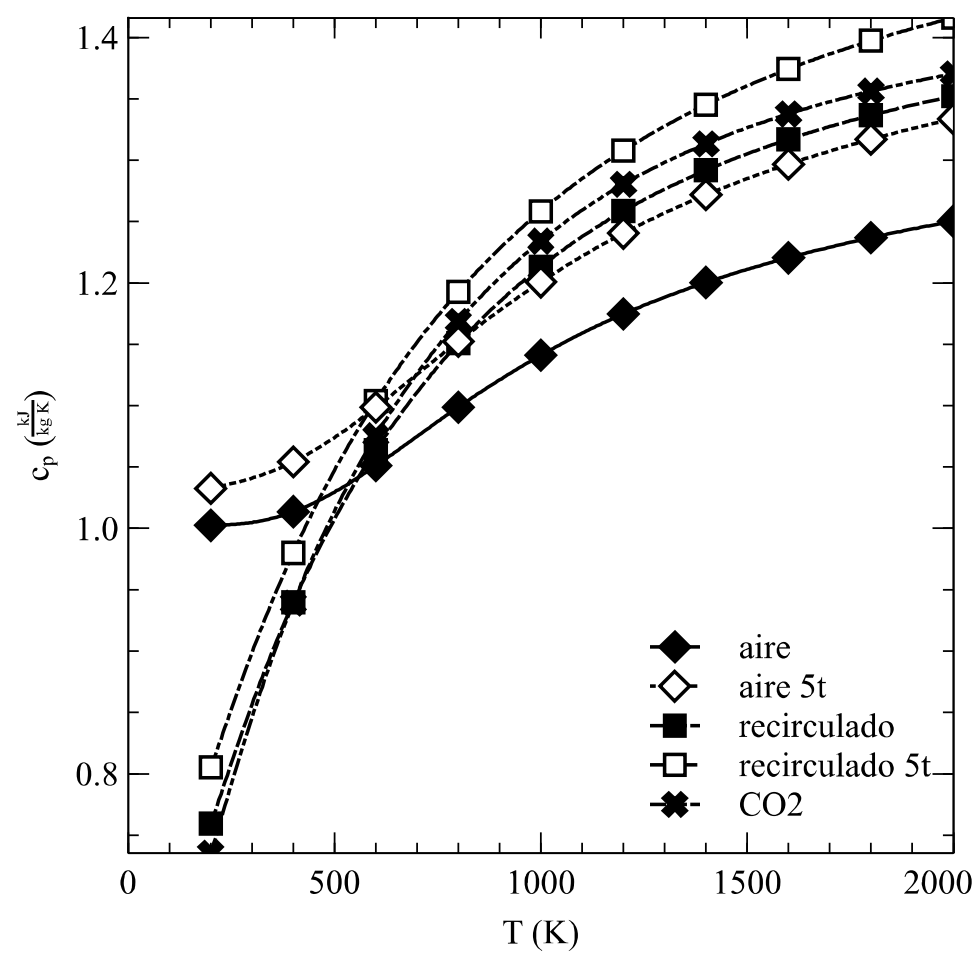

Figura 3. Variación con la temperatura de la capacidad térmica específica a presión a presión constante de los gases de la Tabla 13.

La relación de calores específicos, $\gamma$, para la composición de equilibrio de cada gas a la temperatura correspondiente, se muestra en la Figura 4. Como se verá a continuación, esta propiedad tiene un efecto considerable sobre las actuaciones de la turbina de gas, especialmente sobre la relación de presiones de rendimiento máximo. En este caso la diferencia entre los casos con aire y los casos ricos en $\mathrm{CO}_{2}$ es considerable y se incrementa aún más con la temperatura. Para esta variable hay poca diferencia entre el gas recirculado y el gas recirculado $5 t$, pero sí hay diferencia entre estos casos y el $\mathrm{CO}_{2}$ puro. 


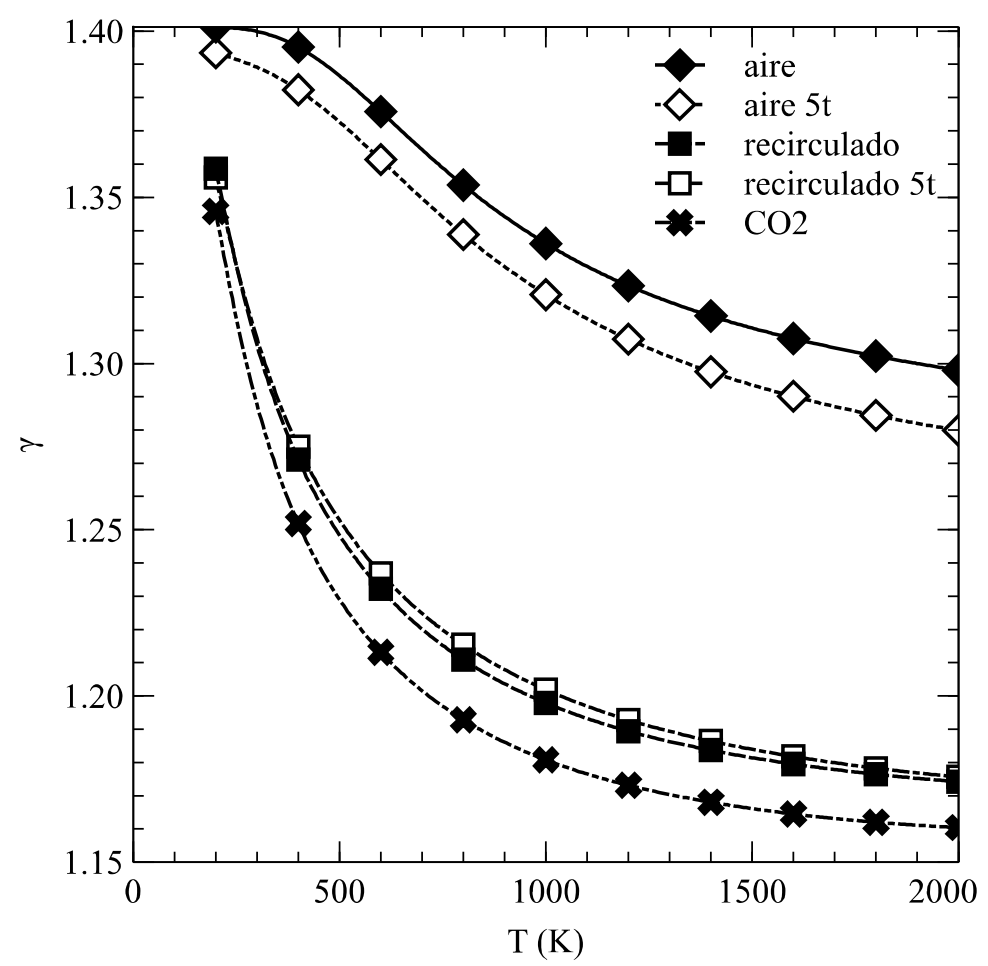

Figura 4. Variación con la temperatura de la relación de calores específicos de los gases de la Tabla 13.

\subsection{Análisis simplificado del sistema.}

En la Figura 5 se ha representado de manera simplificada un ciclo combinado de gas y vapor. Las actuaciones de la turbina de gas pueden obtenerse de manera simplificada suponiendo que el fluido de trabajo es un gas ideal, con una capacidad térmica específica a presión constante, $c_{p}$ y una relación de capacidades térmicas $\gamma$, constantes. Para simplificar la notación, la relación de temperaturas totales entre la estaciones $i$ y $j$ se escribirá como

$$
\theta_{i j}=\frac{T_{j t}}{T_{i t}},
$$

y la relación de presiones totales entre las mismas estaciones como

$$
\pi_{i j}=\frac{P_{j t}}{P_{i t}} .
$$




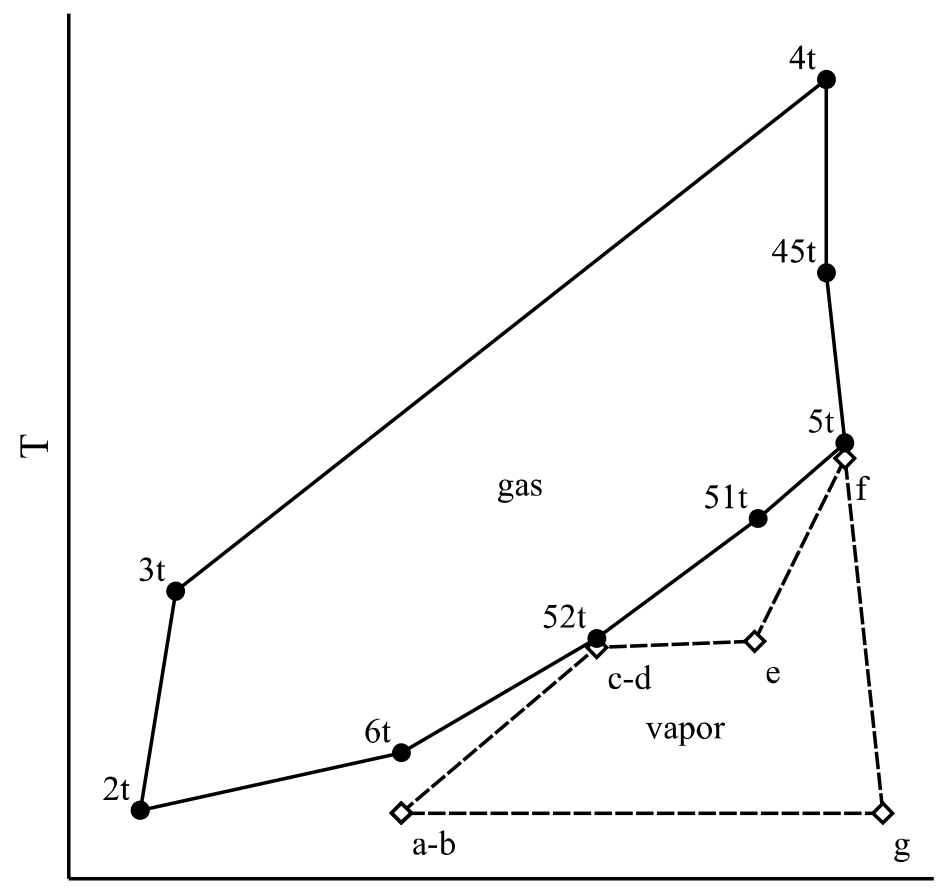

S

Figura 5. Representación aproximada de un ciclo combinado.

El compresor, encargado de aumentar la presión de remanso entre las estaciones 2 t y $3 t$, se caracterizará por la relación de presiones

$$
\pi_{23}=\frac{P_{3 t}}{P_{2 t}}
$$

Si se considera como referencia una compresión adiabática e isentrópica hasta la estación 3st, su relación de temperaturas totales vendrá dada por

$$
\theta_{23 s}=\frac{T_{3 s t}}{T_{2 t}}=\pi_{23}^{\frac{\gamma-1}{\gamma}}
$$

La relación de temperaturas para una compresión no isentrópica puede definirse mediante el rendimiento isentrópico

$$
\eta_{23}=\frac{\theta_{23 s}-1}{\theta_{23}-1}
$$


Aunque el uso del rendimiento isentrópico hace algo más sencillas las expresiones, y es el usado en este tipo de análisis en la literatura, su valor depende de la relación de presiones para un nivel tecnológico determinado. En este sentido sería mejor emplear el rendimiento politrópico, que se considera que depende únicamente del estado del arte en el diseño de la turbomaquinaria (el nivel tecnológico), pero los resultados son cualitativamente similares en ambos casos, por lo que se ha decidido seguir la práctica más común. La potencia necesaria para la compresión se puede escribir como

$$
W_{23}=m_{23} c_{p}\left(T_{3 t}-T_{2 t}\right)=m_{23} c_{p} T_{2 t}\left(\theta_{23}-1\right)
$$

Para independizar el análisis del ciclo del tamaño del sistema, caracterizado por el gasto másico $m_{23}$, se puede definir una potencia adimensional, $\tau$, que para el compresor tiene la forma

$$
\tau_{23}=\frac{W_{23}}{m_{23} c_{p} T_{2 t}}=\theta_{23}-1
$$

La cámara de combustión se caracteriza mediante la temperatura final de combustión $T_{4 t}$. Se hacen las suposiciones adicionales de combustión perfecta (toda la energía del combustible se emplea en aumentar la temperatura del gas), combustión diluida (el gasto de aire es mucho mayor que el de combustible, por lo que este se desprecia), y ausencia de sangrado del aire del compresor. Bajo estas condiciones, el gasto másico de gas se mantiene constante a lo largo del ciclo, siendo igual al del compresor.

$$
m_{45}=m_{34}=m_{23}=m_{C G}
$$

La potencia añadida al gas en la cámara de combustión se puede expresar en la forma

$$
W_{34}=m_{C G} c_{p}\left(T_{4 t}-T_{3 t}\right)
$$

y en forma adimensional 


$$
\tau_{34}=\frac{W_{34}}{m_{23} c_{p} T_{2 t}}=\theta_{24}-\theta_{23}=\theta_{24}-1+\frac{1-\theta_{23 s}}{\eta_{23}}
$$

La turbina se caracteriza fijando una presión de salida igual a la de entrada al compresor, con lo que su relación de presiones será la inversa de la del compresor, si se asume que no hay pérdidas en la cámara de combustión.

$$
\pi_{45}=\frac{1}{\pi_{23}}
$$

Si se define una expansión isentrópica hasta el estado 5st mediante

$$
\theta_{45 s}=\pi_{45}^{\frac{\gamma-1}{\gamma}}=\left(\frac{1}{\pi_{23}}\right)^{\frac{\gamma-1}{\gamma}}=\frac{1}{\theta_{23 s}}
$$

El estado final en una expansión no isentrópica también puede definirse mediante el rendimiento isentrópico

$$
\eta_{45}=\frac{\theta_{45}-1}{\theta_{45 s}-1}
$$

La potencia extraída de la turbina será, entonces

$$
W_{45}=m_{C G} c_{p}\left(T_{4 t}-T_{5 t}\right)
$$

En forma adimensional

$$
\tau_{45}=\frac{W_{45}}{m_{C G} c_{p} T_{2 t}}=\theta_{24}\left(1-\theta_{45}\right)=\theta_{24} \eta_{45 s}\left(1-\theta_{45 s}\right)=\theta_{24} \eta_{45 s}\left(1-\frac{1}{\theta_{23 s}}\right)
$$

La potencia neta extraída del ciclo de gas será

$$
W_{C G}=W_{45}-W_{23}
$$

En forma adimensional 


$$
\tau_{C G}=\frac{W_{C G}}{m_{C G} c_{p} T_{2 t}}=\tau_{45}-\tau_{23}=\theta_{24} \eta_{45 s}\left(1-\frac{1}{\theta_{23 s}}\right)-\frac{\theta_{23 s}-1}{\eta_{23}}
$$

Dado que el área frontal y, por lo tanto, el tamaño característico de la turbina de gas, y su coste, están directamente relacionados con el gasto másico que pasa por ella, la relación entre la potencia extraída y un gasto másico característico es un resultado fundamental de sus actuaciones, denominado potencia específica. La potencia específica se deduce directamente de la potencia adimensional obtenida anteriormente.

$$
\omega_{C G}=\frac{W_{C G}}{m_{C G}}=c_{p} T_{2 t} \tau_{C G}
$$

Resulta de interés el análisis del comportamiento de la potencia específica con los parámetros característicos del ciclo, $\pi_{23}$ y $T_{4 t}$, y con la composición del gas de trabajo, reflejada en los parámetros $\gamma$ y $c_{p}$. En concreto, se suele buscar el máximo de la potencia específica con la relación de presiones, que se puede obtener a partir de la expresión de la potencia adimensional. Puede verse que la potencia adimensional depende de la relación de presiones a través de la variable $\theta_{23 s}$, y solo depende de las propiedades del fluido a través de esta variable. El máximo puede obtenerse mediante:

$$
\begin{aligned}
& \frac{\partial \tau_{C G}}{\partial \theta_{23 s}}=\frac{\theta_{24} \eta_{45}}{\theta_{23 s}^{2}}-\frac{1}{\eta_{23}}=0 \\
& \theta_{23 s}\left(\tau_{C G, \max }\right)=\sqrt{\eta_{23} \eta_{45} \theta_{24}}
\end{aligned}
$$

Con lo que se tiene

$$
\pi_{23 s}\left(\tau_{C G, \max }\right)=\left(\eta_{23} \eta_{45} \theta_{24}\right)^{\frac{\gamma}{2(\gamma-1)}}
$$




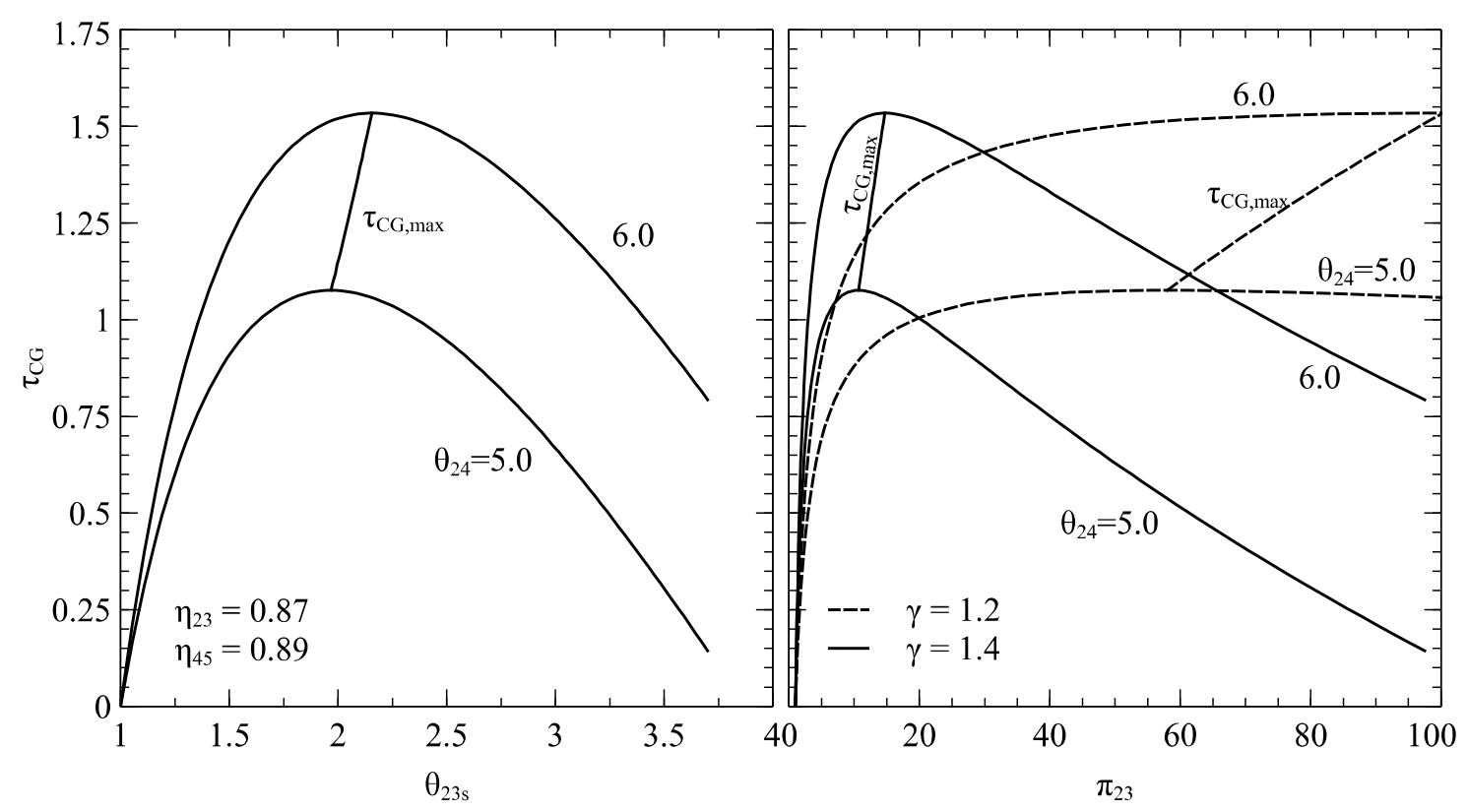

Figura 6. Variación del trabajo específico de un ciclo de gas con $\theta_{23 s}$ (izquierda) y $\pi_{23}$ (derecha) para $\eta_{23}=0.87, \eta_{45}=0.89$ y distintos valores de $\theta_{24}$ y $\gamma$.

Las expresiones de la potencia específica del ciclo de gas en función de $\theta_{23 \mathrm{~s}}$ y $\pi_{23}$ se han representado en la Figura 6, para $\eta_{23}=0.87, \eta_{45}=0.89$ y distintos valores de $\theta_{24} \mathrm{y} \gamma$. El efecto de $\gamma$ es muy importante, mientras que para $\gamma=1.4$ la potencia específica máxima se alcanza para valores bajos de la relación de presiones, variando poco con $\theta_{24}$, para $\gamma=1.2$ la relación de presiones del máximo se desplaza hacia valores muy altos, y tiene una fuerte dependencia de $\theta_{24}$. Es interesante apreciar que las curvas para $\gamma=1.2$ son muy planas en un rango considerable de $\pi_{23}$.

Otro resultado fundamental es el rendimiento del ciclo de gas, expresado como la relación entre el trabajo del ciclo de gas y la potencia añadida al sistema mediante el combustible, expresada en la forma simplificada $W_{34}$. La expresión del rendimiento puede obtenerse mediante las potencias adimensionales correspondientes, en la forma:

$$
\eta_{C G}=\frac{\tau_{C G}}{\tau_{34}},
$$

obteniendo 


$$
\eta_{C G}=\frac{\theta_{24} \eta_{45 s}\left(1-\frac{1}{\theta_{23 s}}\right)-\frac{\theta_{23 s}-1}{\eta_{23}}}{\theta_{24}-1+\frac{1-\theta_{23 s}}{\eta_{23}}} .
$$

A partir de la condición de máximo

$$
\frac{\partial \eta_{C G}}{\partial \theta_{23 s}}=0
$$

se obtiene la expresión

$$
\theta_{23 s}^{2}\left(\eta_{45} \theta_{24}-\theta_{24}+1\right)-2 \eta_{45} \theta_{24} \theta_{23 s}+\eta_{45} \theta_{24}\left(\eta_{23} \theta_{24}+1-\eta_{23}\right)=0
$$

Y el máximo buscado es la menor de las soluciones

$$
\begin{gathered}
\theta_{23 s}\left(\eta_{C G, \max }\right)=\frac{\eta_{45} \theta_{24}-\sqrt{\eta_{45} \theta_{24}} \sqrt{\theta_{24}^{2}\left(-\eta_{23} \eta_{45}+\eta_{23}\right)+\theta_{24}\left(\eta_{23} \eta_{45}-2 \eta_{23}+1\right)+\eta_{23}-1}}{1-\theta_{24}+\eta_{45} \theta_{24}} . \\
\pi_{23 s}\left(\eta_{C G, \max }\right)=\left(\theta_{23 s}\left(\eta_{C G, \max }\right)\right)^{\frac{\gamma}{\gamma-1}}
\end{gathered}
$$

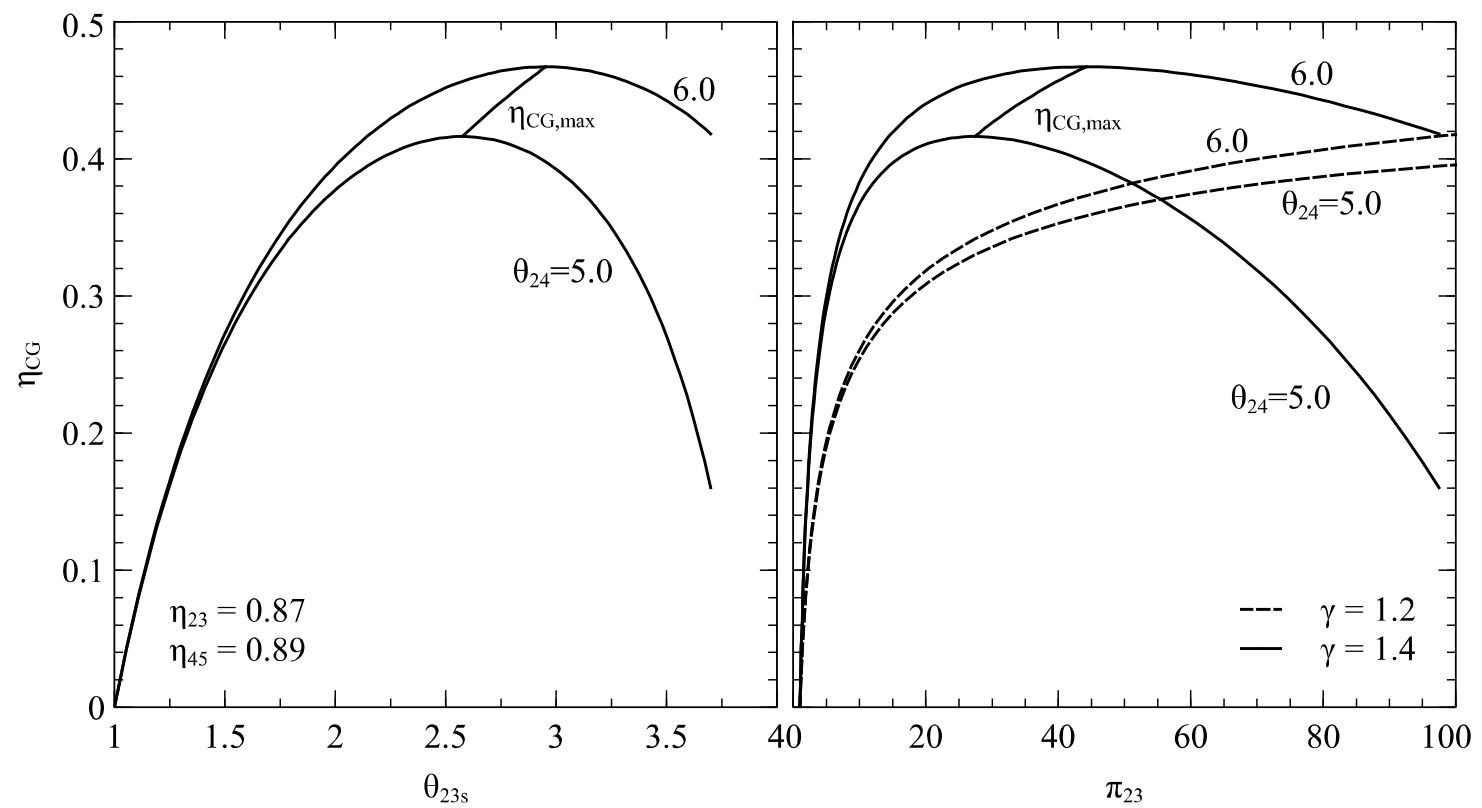

Figura 7. Variación del rendimiento de un ciclo de gas con $\theta_{23 s}$ (izquierda) y $\pi_{23}$ (derecha) para $\eta_{23}$ $=0.87, \eta_{45}=0.89$ y distintos valores de $\theta_{24}$ y $\gamma$. 
La Figura 7 muestra como varía el rendimiento del ciclo de gas con $\theta_{23 \mathrm{~s}}$ y $\pi_{23}$, para $\eta_{23}=0.87, \eta_{45}=0.89$ y distintos valores de $\theta_{24} \mathrm{y} \gamma$. El efecto de $\gamma$ es todavía más significativo, mientras que para $\gamma=1.4$ la potencia específica máxima se alcanza para valores relativamente bajos de $\pi_{23}$, con ciertas variaciones $\operatorname{con} \theta_{24}$, para $\gamma=1.2$ la relación de presiones del máximo no se muestra en el rango representado, además, las curvas cambian poco con $\theta_{24}$.

A la potencia obtenida del ciclo de gas hay que sumar la potencia obtenida del ciclo de vapor, que puede expresarse en la forma:

$$
W_{C V}=m_{C V}\left(h_{f}-h_{g}\right)
$$

Donde $m_{C V}$ es el gasto másico de agua del ciclo de vapor, $h_{f}$ y $h_{g}$ son las entalpías por unidad de gasto másico a la entrada y a la salida de la turbina de vapor, respectivamente, y se ha despreciado el consumo de potencia de la bomba. La entalpia $h_{f}$ vendrá fijada por $T_{f}$, que viene dada por la temperatura máxima admisible o por $T_{5 t} \mathrm{y}$ el salto de temperatura fijado en dicho punto. La entalpía $h_{g}$ depende de las condiciones a la salida de la turbina, como el título de vapor.

La potencia del ciclo de vapor se puede expresar en forma adimensional como

$$
\tau_{C V}=\frac{W_{C V}}{m_{C G} c_{p} T_{2 t}}=\frac{m_{C V}}{m_{C G}}\left(\frac{h_{f}-h_{g}}{c_{p} T_{2 t}}\right) .
$$

Mientras que la relación entre los gastos másicos se puede obtener del balance de potencia transferida del ciclo de gas al ciclo de vapor entre las estaciones $52 \mathrm{t}$ $5 t$, y c-f, que puede expresarse en la forma:

$$
m_{C G} c_{p}\left(T_{5 t}-T_{52 t}\right)=m_{C V}\left(h_{f}-h_{c}\right)
$$

de donde se obtiene: 


$$
\frac{m_{C V}}{m_{C G}}=\frac{c_{p}\left(T_{5 t}-T_{52 t}\right)}{h_{f}-h_{c}}=\frac{\theta_{25}-\theta_{252}}{\frac{h_{f}-h_{c}}{c_{p} T_{2 t}}} .
$$

Si el punto de mínima diferencia de temperatura entre los ciclos, $\Delta T_{\text {pinch }}$, se produce entre las estaciones $52 \mathrm{t}$ y d, $T_{52 t}$ será función de la temperatura de evaporación del ciclo de vapor $T_{d}$, y de $\Delta T_{\text {pinch }}$ luego:

$$
\theta_{252}=\frac{T_{d}+\Delta T_{\text {pinch }}}{T_{2 t}}
$$

De donde se obtiene:

$$
\tau_{C V}=\left(\theta_{25}-\frac{T_{d}+\Delta T_{\text {pinch }}}{T_{2 t}}\right)\left(\frac{h_{f}-h_{g}}{h_{f}-h_{c}}\right)
$$

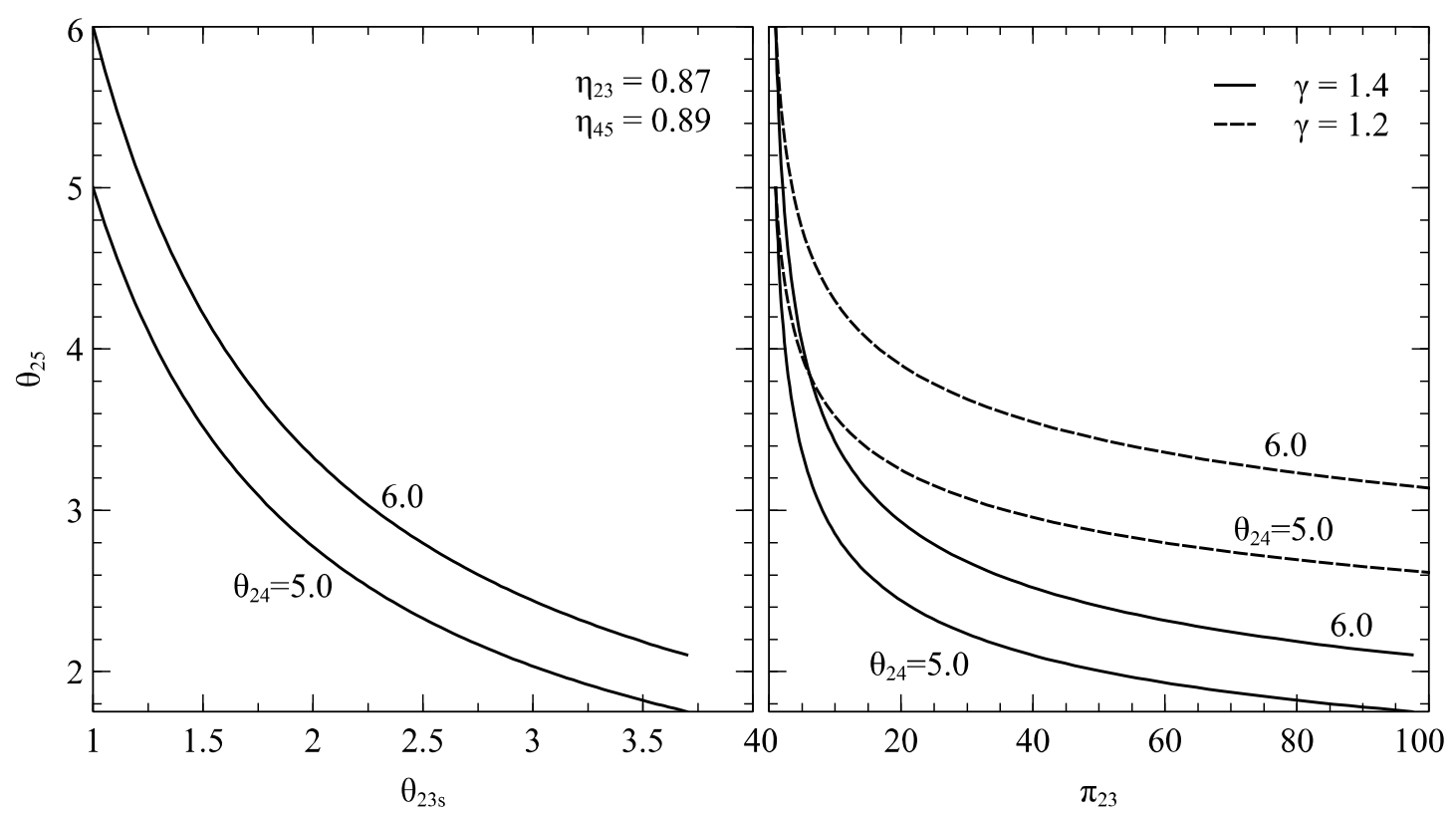

Figura 8. Variación de la temperatura adimensional a la salida de la turbina de gas con $\theta_{23 s}$ (izquierda) y $\pi_{23}$ (derecha) para $\eta_{23}=0.87, \eta_{45}=0.89$ y distintos valores de $\theta_{24} y \gamma$.

La expresión de la potencia específica del ciclo de vapor no se va a analizar con más detalle, aunque cualitativamente se aprecia que depende fundamentalmente de la variable $\theta_{25}$, que tiene la forma: 


$$
\theta_{25}=\theta_{24} \theta_{45}=\theta_{24}\left(1-\eta_{45 s}\left(1-\frac{1}{\theta_{23 s}}\right)\right)
$$

La temperatura a la salida de la turbina de gas cae con $\theta_{23 s}$ y $\pi_{23}$, como se muestra en la Figura 8. La manera en que cae con $\pi_{23}$ depende considerablemente del valor de $\gamma$.

También puede obtenerse el rendimiento del ciclo de vapor, en la forma:

$$
\eta_{C V}=\frac{\tau_{C V}}{\tau_{34}}
$$

De nuevo, el análisis de cómo varía este rendimiento se llevará a cabo en el modelo detallado.

La potencia neta obtenida del CCSC será la potencia total obtenida del ciclo combinado, menos el consumo de la ASU y del CCS:

$$
W_{\text {neto }}=W_{C C}-W_{A S U}-W_{C C S} .
$$

Se puede suponer que el consumo de la ASU es proporcional al gasto másico generado por ella, con una constante de proporcionalidad $K_{A S U}$, mientras que el consumo del sistema CCS también se supondrá proporcional al gasto másico que pasa por él, con una constante $K_{C C S}$. La eficiencia neta basada en el poder calorífico inferior del combustible puede escribirse en la forma

$$
\begin{gathered}
\eta_{\text {neta }}=\frac{W_{C C}}{m_{F} L H V}-\frac{m_{A S U}}{m_{F}} \frac{K_{A S U}}{L H V}-\frac{m_{C C S}}{m_{F}} \frac{K_{C C S}}{L H V}=\eta_{C C}-\Delta \eta_{A S U}-\Delta \eta_{C C S} \\
m_{P, \text { est }}=m_{F}+m_{A S U, \text { est }}=\left(1+f_{\text {est }}\right) \varphi m_{A S U}
\end{gathered}
$$

Usando la relación entre el gasto másico del CCS y del ASU, y la relación

$$
m_{F}=\varphi f_{e s t} m_{A S U},
$$


la eficiencia neta se puede obtener como

$$
\eta_{\text {neta }}=\eta_{C C}-\frac{K_{A S U}+z K_{C C S}}{\varphi f_{\text {est }} L H V}
$$

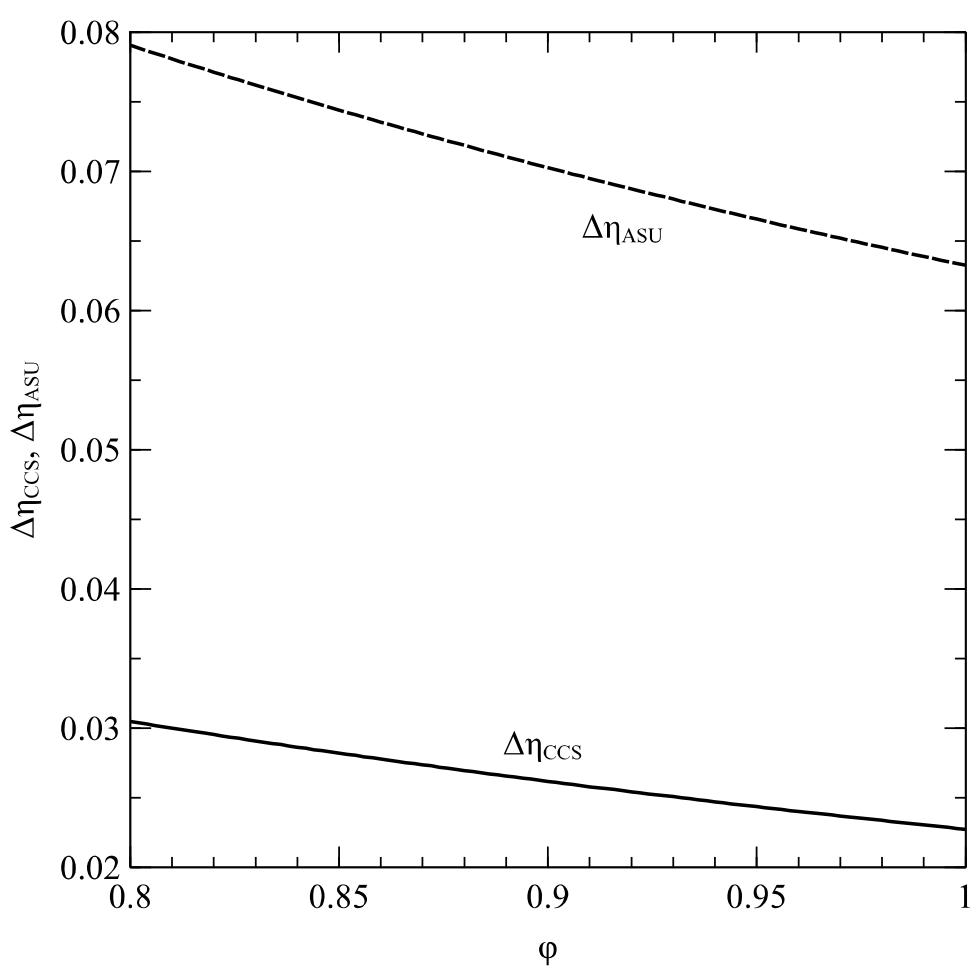

Figura 9. Variación de $\Delta \eta_{A S U} y \Delta \eta_{C C S} \operatorname{con} \varphi$.

Como se muestra en la Figura 9, tanto $\Delta \eta_{A S U}, \Delta \eta_{C C S}$ disminuyen cuando $\varphi$ tiende 1. Sin embargo el proceso de combustión requiere cierto exceso de oxidante, como se mostrará en la sección correspondiente a la cámara de combustión, por lo que se ha usado $\varphi=0.98$. En la Tabla 14 se comparan los valores obtenidos para para $\varphi=1 \mathrm{y} \varphi=0.98$, donde se ve que la diferencia en las pérdidas por usar $\varphi=0.98$ es relativamente pequeña. En cualquier caso, suponen una penalización muy importante para el CCSC.

\begin{tabular}{ccc}
\hline$\varphi$ & $\Delta \eta_{\text {ASU }}$ & $\Delta \eta_{\mathrm{CCS}}$ \\
\hline 1.0 & 0.0632 & 0.0227 \\
0.98 & 0.0645 & 0.0234 \\
$\Delta(\%)$ & 2.0 & 2.8 \\
\hline
\end{tabular}

Tabla 14.Valores de $\Delta \eta_{\text {ASU }}$ y $\Delta \eta_{C C S}$ para $\varphi=1$ y $\varphi=0.98$. 


\subsection{Análisis paramétrico.}

En la Figura 10 se puede ver la influencia de la relación de presiones del ciclo de gas en el rendimiento del ciclo de gas, del ciclo de vapor y del sistema completo, tanto para el ciclo combinado semicerrado como para el ciclo combinado estándar, que se usa como base de comparación, para una temperatura fin de combustión $T_{4 t}=1600 \mathrm{~K}$ y un gasto másico de refrigeración del $20 \%$ del gasto a la salida del compresor, en adelante caso 1600K $20 \%$.

Para el ciclo combinado estándar, funcionando con aire, el rendimiento máximo se obtiene para una relación de presiones en torno a 13, aunque el ciclo de gas alcanza un máximo en torno a la relación de presiones 35. La variación del rendimiento del ciclo combinado es pequeña en el entorno de relación de presiones entre 10 y 20, cayendo algo más para valores superiores. Como puede verse en la Tabla 5, la relación de presiones empleada en las turbinas actuales está en este rango, siendo mayor para las turbinas aeroderivadas.

El ciclo combinado semicerrado presenta varias diferencias con el ciclo combinado estándar:

- La relación de presiones óptima del ciclo de ciclo combinado es mayor (en torno a 36), aunque presenta variaciones muy pequeñas en el rango 2550.

- El rendimiento máximo es mayor en el rango considerado, con un 0.563 frente a un 0.540 del ciclo estándar (cerca de un 4\% mayor).

- Si se incluyen las pérdidas debidas a la ASU y al sistema de captura de $\mathrm{CO}_{2}$ se obtiene una eficiencia máxima de 0.476 , un $15 \%$ inferior a las obtenidas sin estas pérdidas. Más de tres cuartas partes de dichas pérdidas son debidas al consumo de la ASU.

La tendencia del rendimiento del ciclo de gas es similar a la que predice el modelo simplificado basado en el cambio de propiedades del gas. 


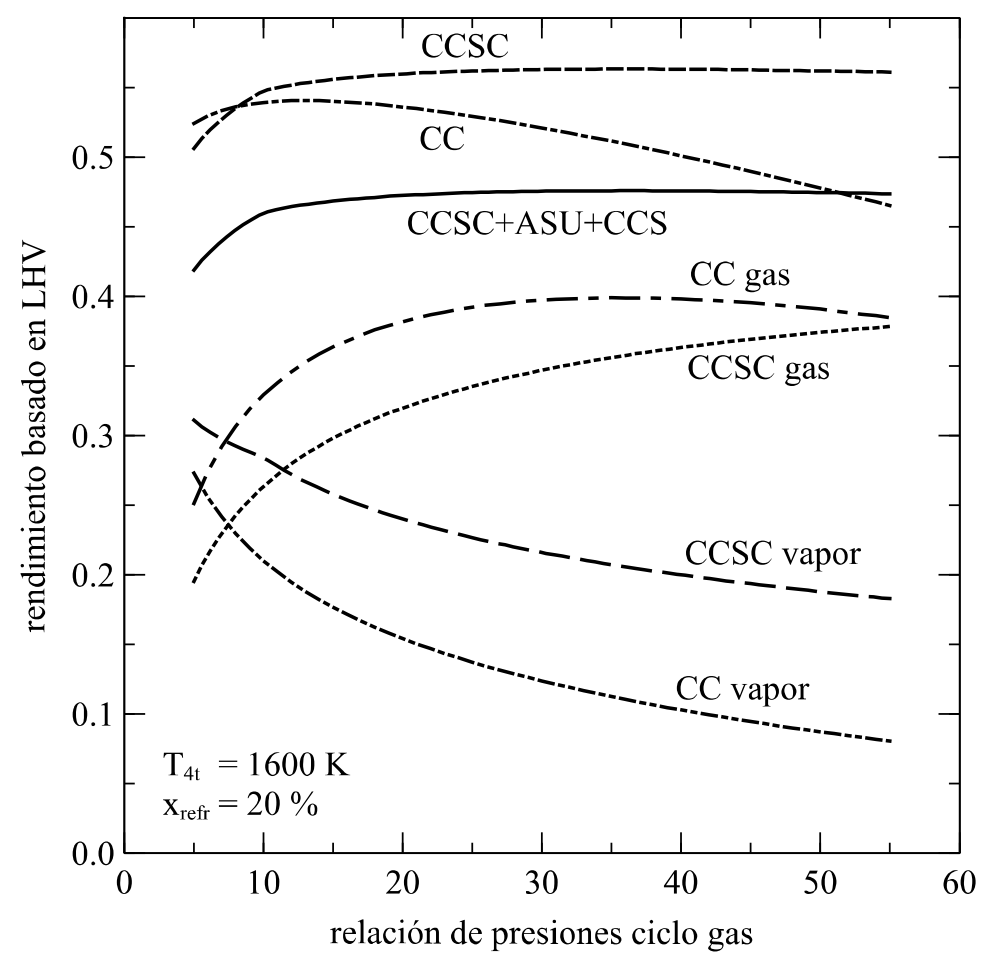

Figura 10. Variación de la eficiencia del ciclo combinado semicerrado y un ciclo combinado estándar con la relación de presiones del ciclo de gas, para $T_{4 t}=1600 \mathrm{~K}, x_{\text {refr }}=20 \%$.

También se aprecia un brusco descenso en el rendimiento del ciclo combinado semicerrado por debajo de relación de presiones 10. Este efecto tiene su origen en el generador de vapor, como puede verse en la Figura 11. En esta figura se ha representado la evolución de la temperatura y la entalpía adimensionalizada con la entalpía total transferida en el generador de vapor para tres relaciones de presión distintas del lado gas (5, 10 y 15 bar), y se han comparado con la evolución del ciclo de vapor. Puede verse que el punto de mínima diferencia de temperatura entre los ciclos, que usualmente se asume que está situado en la estación de entrada del evaporador del ciclo de vapor, se desplaza a la entrada del economizador del ciclo de vapor, es decir, la salida del economizador del ciclo de gas. Este desplazamiento está relacionado con la alta temperatura que se alcanza a la salida de la turbina de gas para relaciones de compresión bajas y a la variación con la temperatura de la capacidad térmica a presión constante del gas. 


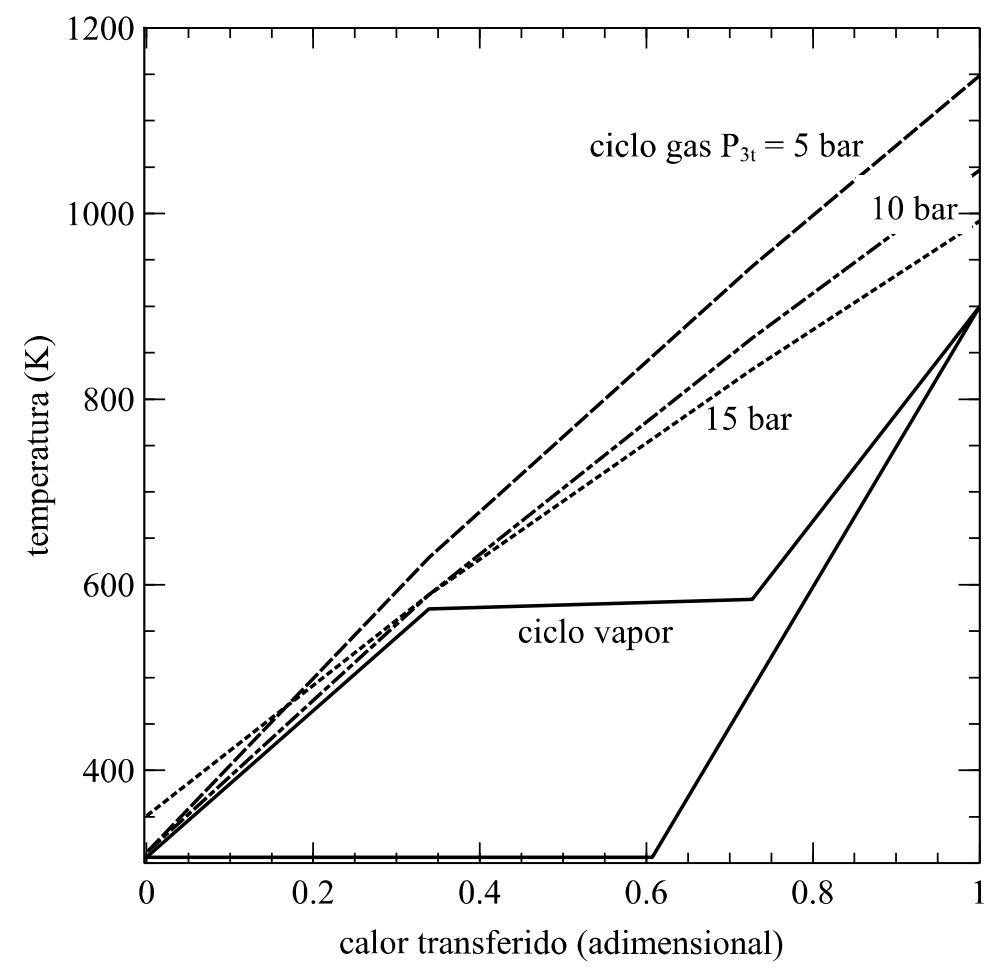

Figura 11. Evolución del calor transferido entre el ciclo de vapor y el ciclo combinado semicerrado, con $T_{4 t}=1600 \mathrm{~K}, x_{\text {refr }}=20 \%$ y distintos valores de relación de presiones.

El comportamiento del ciclo de vapor viene determinado por la temperatura máxima de dicho ciclo, $T_{v}$, que está ligada a la temperatura a la salida de la turbina del ciclo de gas, $T_{5 t}$, cuya variación con la relación de presiones se muestra en la Figura 12. En esta figura se ve que $T_{5 t}$ es mucho mayor en el ciclo semicerrado que en el ciclo con aire para una misma relación de presiones. De este modo, aunque la eficiencia del ciclo semicerrado es inferior a la del ciclo de aire, la energía disponible para el ciclo de vapor asociado es mucho mayor, de modo que el rendimiento del ciclo combinado acaba siendo del mismo orden. Sin embargo, la turbina del ciclo de vapor no admite las altas temperaturas a la salida de la turbina del ciclo de gas, habiéndose considerado un límite de $900 \mathrm{~K}$ correspondiente a $T_{5 t}=925 \mathrm{~K}$, por lo que en la zona con relaciones de presión menores de 26 es necesario reducir la eficiencia del generador de vapor.

La potencia específica, definida como potencia neta dividida entre el gasto másico a la salida de la turbina de gas, es otro resultado fundamental para su diseño, dado que una potencia específica mayor se traduce en un sistema más 
pequeño para una potencia dada, lo que está ligado con menores costes de la instalación.

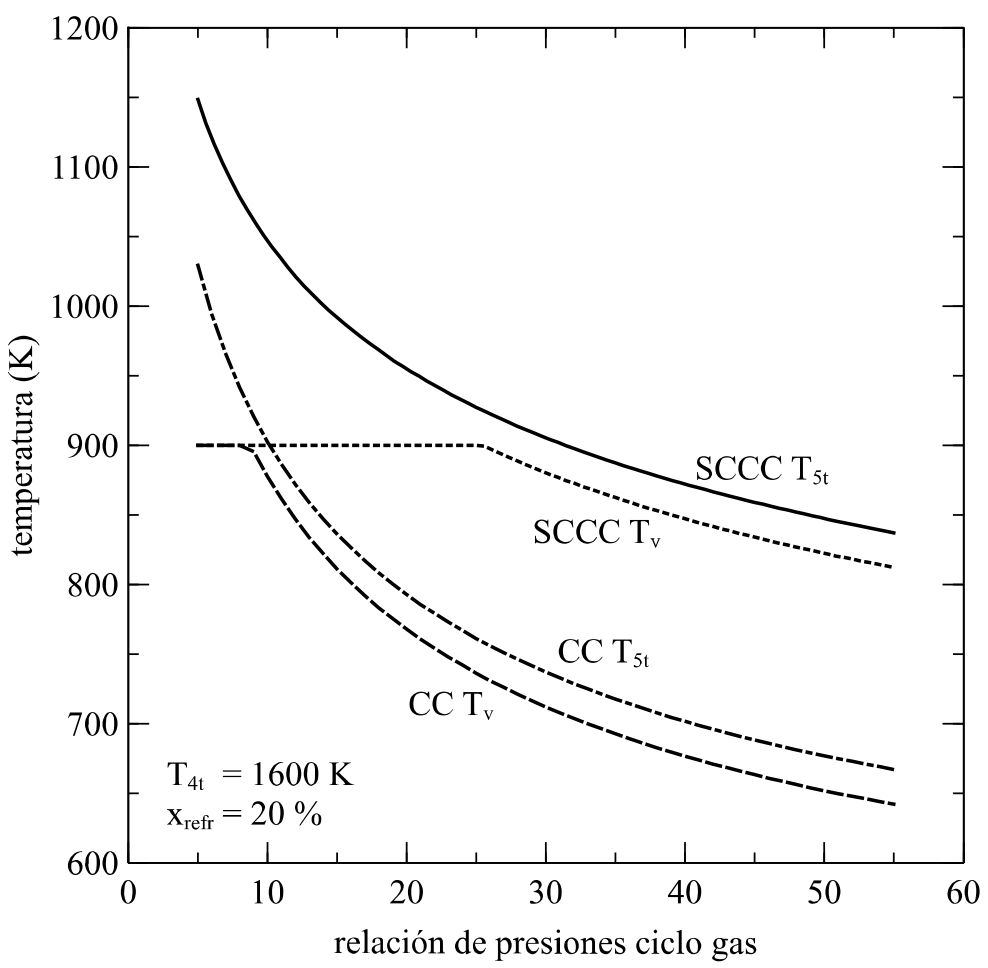

Figura 12. Variación de las temperaturas a la salida de la turbina de gas y entrada de la turbina de vapor del ciclo combinado semicerrado y el ciclo combinado estándar con la relación de presiones del ciclo de gas, para $T_{4 t}=1600 \mathrm{~K}, x_{\text {refr }}=20 \%$.

La variación de la potencia específica con la relación de presiones se muestra en la Figura 13, donde se ve que la potencia específica del ciclo combinado semicerrado es mayor que la del ciclo combinado estándar, incluyendo las pérdidas debidas a la ASU y al sistema de captura de $\mathrm{CO}_{2}$, para relaciones superiores a 14.

Para el ciclo semicerrado, una relación de presiones tan baja como 20 tiene un rendimiento solo un $0.6 \%$ inferior al máximo, con una ganancia de casi un $6.9 \%$ en potencia específica; para relación de presiones 30 se tiene menos de un $0.1 \%$ de pérdida en rendimiento con una ganancia del $2.4 \%$ de potencia específica. En este caso puede estar justificado fijar una relación de presiones de diseño inferior a la de máximo rendimiento, con una pequeña pérdida de rendimiento y un considerable aumento de la potencia específica. 


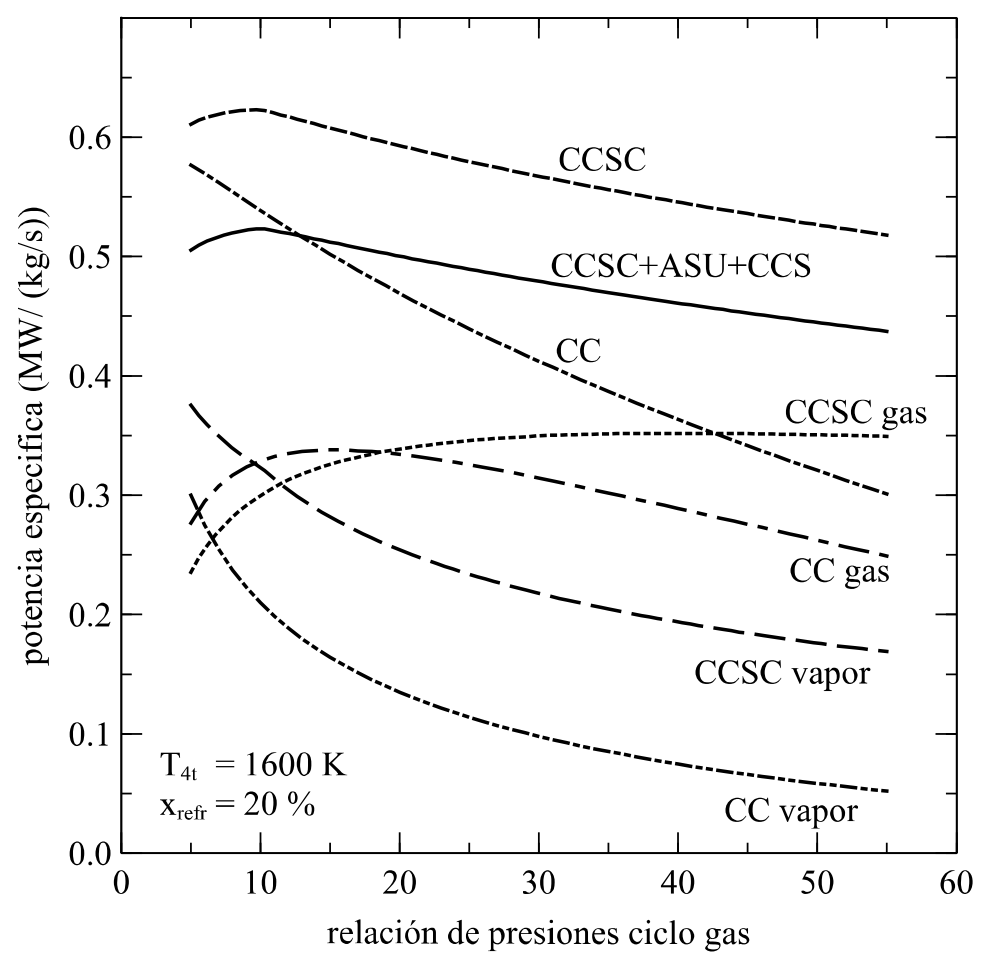

Figura 13. Variación de la potencia específica del ciclo combinado semicerrado y el ciclo combinado estándar con la relación de presiones del ciclo de gas, para $T_{4 t}=1600 \mathrm{~K}, x_{\text {refr }}=20 \%$.

La diferencia entre el ciclo combinado estándar y el semicerrado se puede descomponer en las correspondientes diferencias entre los ciclos de gas y vapor:

- Por una parte, la relación de presiones de máxima potencia específica del ciclo de gas del ciclo semicerrado está en torno a 38, mientras que la del ciclo de gas del ciclo combinado estándar está en torno a 15, dado que la potencia específica combinada del ciclo semicerrado cae más lentamente. Este es el efecto esperado del modelo simplificado basado en el cambio de propiedades del gas.

- Por otra, la potencia específica suministrada por el ciclo de vapor del ciclo semicerrado es muy superior a la del ciclo estándar. Además del efecto de la mayor temperatura de salida de la turbina que se ha mostrado en la Figura 12, la relación de gasto másico de vapor generado entre la de gas es mayor en el ciclo semicerrado, como se puede ver en la Figura 14. 


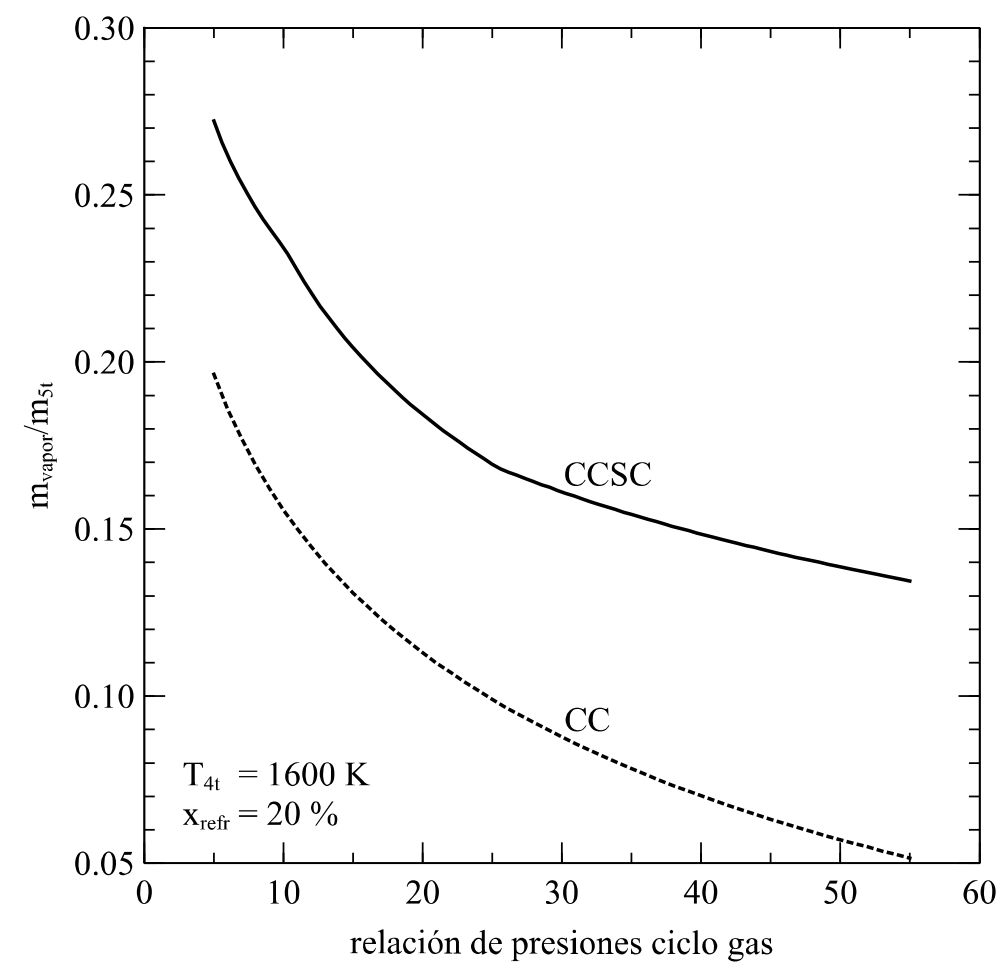

Figura 14. Variación de la relación entre el gasto másico de vapor y el gasto másico de gas a la salida de la turbina del ciclo combinado semicerrado y el ciclo combinado estándar con la relación de presiones del ciclo de gas, para $T_{4 t}=1600 \mathrm{~K}, x_{\text {refr }}=20 \%$.

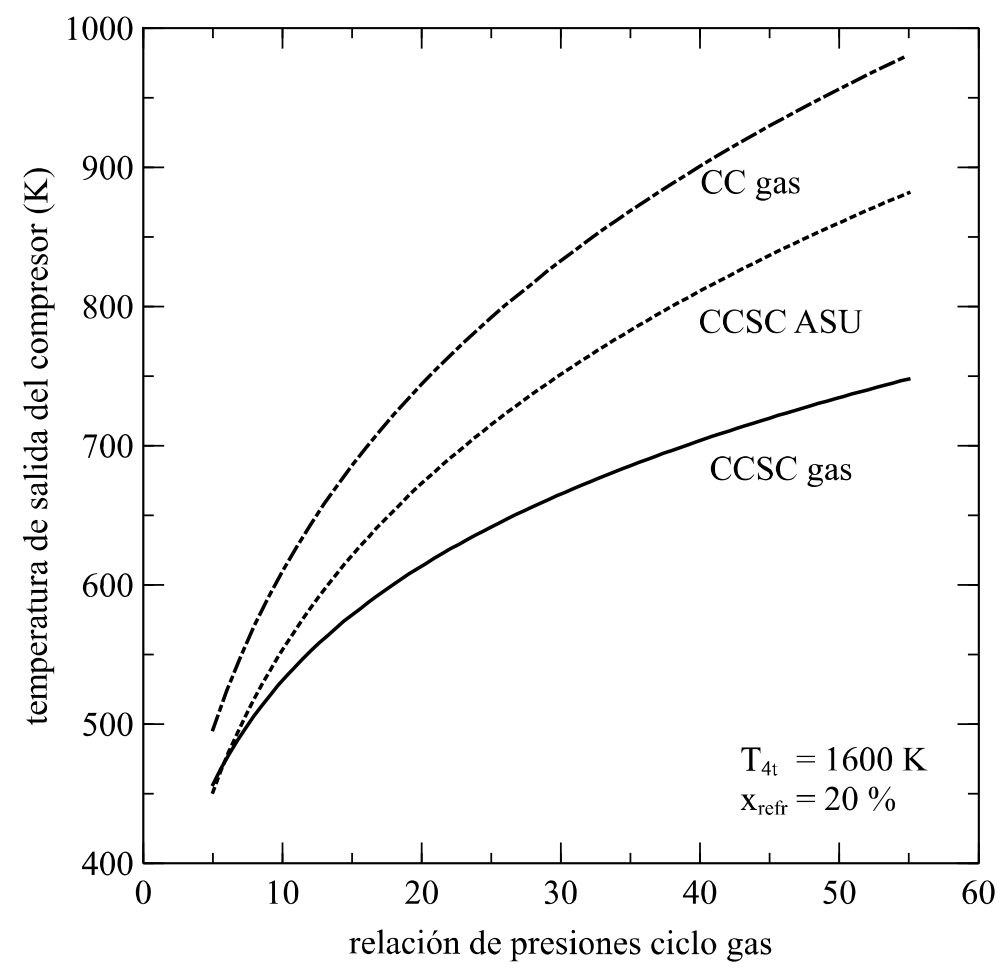

Figura 15. Variación de la temperatura a la salida del compresor del ciclo de aire, del compresor a la salida de la ASU, y del compresor de gas recirculado con la relación de presiones del ciclo de gas, para $T_{4 t}=1600 \mathrm{~K}, x_{\text {refr }}=20 \%$. 
Además de los resultados que caracterizan las actuaciones del ciclo, es necesario comprobar otros resultados que puedan limitar su relación de presiones. Un posible factor limitante del ciclo de gas es la temperatura a la salida de los compresores. En la Figura 15 se presenta la variación con la relación de presiones de la temperatura a la salida de los compresores de gas recirculado y de oxidante, junto con la temperatura a la salida del compresor del ciclo de gas de un ciclo combinado funcionando con aire. Puede apreciarse que las temperaturas obtenidas para el gas recirculado son relativamente bajas en todo el rango de relación de presiones investigado, mientras que las del oxidante son mucho mayores, aunque por debajo de las obtenidas para el aire, de modo que no se prevén problemas con los materiales del compresor debidos a altas temperaturas, al menos para relaciones de presión inferiores a 40.

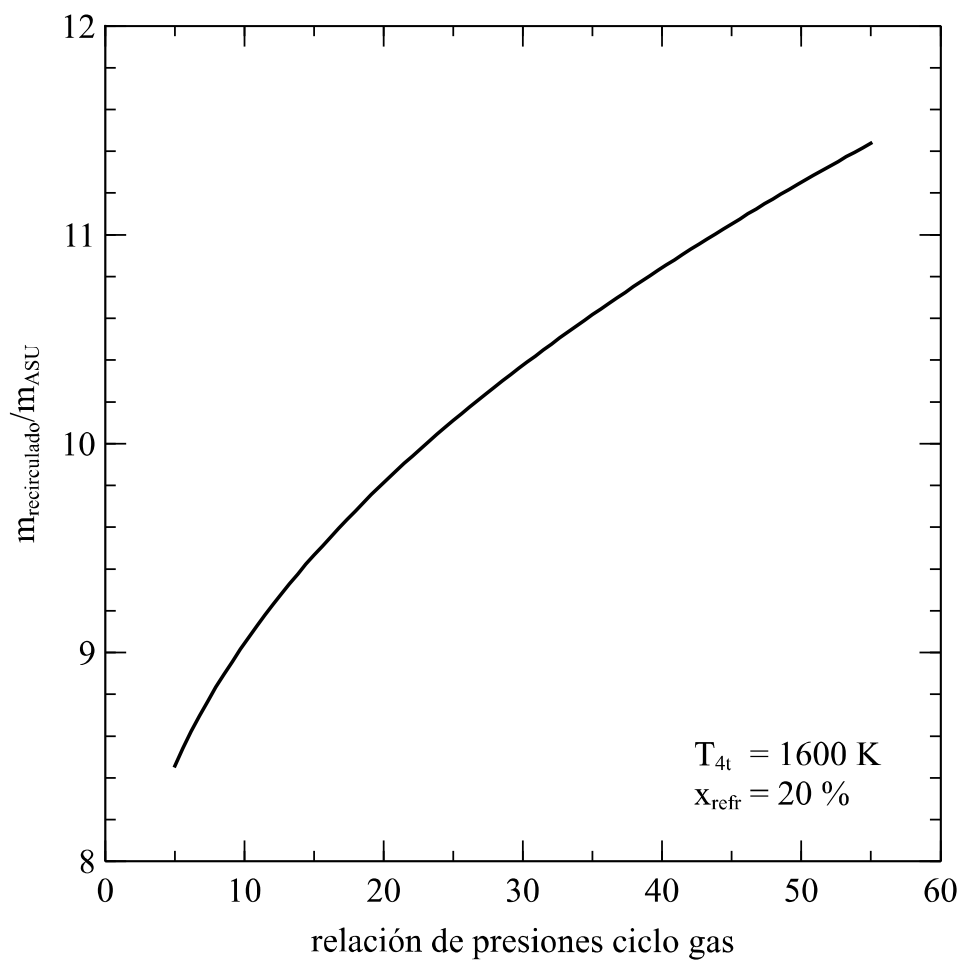

Figura 16. Variación de la relación entre el gasto másico de la ASU y del gasto másico recirculado con la relación de presiones del ciclo de gas, para $T_{4 t}=1600 \mathrm{~K}, x_{\text {refr }}=20 \%$.

Aunque las temperaturas obtenidas para el oxidante no sean excesivamente altas, el uso de oxígeno casi puro podría presentar problemas de seguridad. Dado que la cámara de combustión puede requerir la premezcla del oxidante, 
combustible y una parte del gas recirculado, como se mostrará más adelante, una solución a este problema podría consistir en la mezcla del oxidante con parte del gas recirculado antes de la compresión. La cantidad de gas recirculado disponible varía entre aproximadamente 8.5 y 11.5 veces la de oxidante, como puede verse en la Figura 16.

Una vez mostrado el comportamiento del ciclo ante cambios de la relación de presiones del ciclo de gas, se va a mostrar su comportamiento ante cambios de la temperatura de salida de la cámara de combustión, $T_{4 t}$. Sin embargo, también hay tener en cuenta que el gasto másico necesario para la refrigeración está ligado al valor de $T_{4 t}$, por lo que hay que tener en cuenta ambos efectos de manera simultánea.

En la Figura 17 se compara el rendimiento del ciclo de gas, vapor y sistema completo de un caso con una $T_{4 t}$ de $1600 \mathrm{~K}$ con un $20 \%$ de gasto másico de refrigeración (caso 1600K 20\%) y dos casos a $1700 \mathrm{~K}$, asumiendo dos condiciones distintas para el gasto másico de refrigeración. En uno de los casos se ha supuesto que se mantiene en el $20 \%$, caso $1700 \mathrm{~K} 20 \%$, mientras que en el otro se ha supuesto que es necesario elevarlo al 25\%, manteniendo el mismo reparto entre la refrigeración del rotor y del estátor, caso $1700 \mathrm{~K} 25 \%$. Cada uno de estos casos se ha llevado a cabo para el ciclo combinado semicerrado y para un ciclo combinado estándar, que se usa como base de comparación. En el ciclo combinado semicerrado no se muestra el efecto de la ASU ni del sistema de captura de $\mathrm{CO}_{2}$, por simplicidad. Con este mismo fin, se ha separado la representación de cada uno de los ciclos, utilizando distinto rango y escala en cada eje de ordenadas.

En la parte superior de la Figura 17 se muestra el rendimiento del sistema completo. Para el ciclo con aire se aprecia que el rendimiento máximo del caso $1700 \mathrm{~K} 20 \%$ es un $4.2 \%$ superior al del caso $1600 \mathrm{~K} 20 \%$, pero este aumento es de solo un $1.5 \%$ para el caso $1700 \mathrm{~K} 25 \%$. La relación de presiones de rendimiento máximo cambia muy poco pasando de 13 a 16 en el caso $1700 \mathrm{~K} 20 \%$, y a 14 en 
el caso $1700 \mathrm{~K} 25 \%$. Los cambios del rendimiento del ciclo combinado semicerrado son muy similares, de un $3.9 \%$ para el $1700 \mathrm{~K} 20 \%$ a un $1.8 \%$ para el $1700 \mathrm{~K} 25 \%$. La relación de presiones a la que se produce este valor cambia bastante más, pasando del 36 del $160020 \%$ al 50 para el $1700 \mathrm{~K} 25 \%$ y al 44 para el caso 1700K 20\%. En todos los casos del ciclo semicerrado se aprecia el brusco descenso asociado al desplazamiento del punto de mínima diferencia del generador de vapor a la salida del economizador del ciclo de gas.
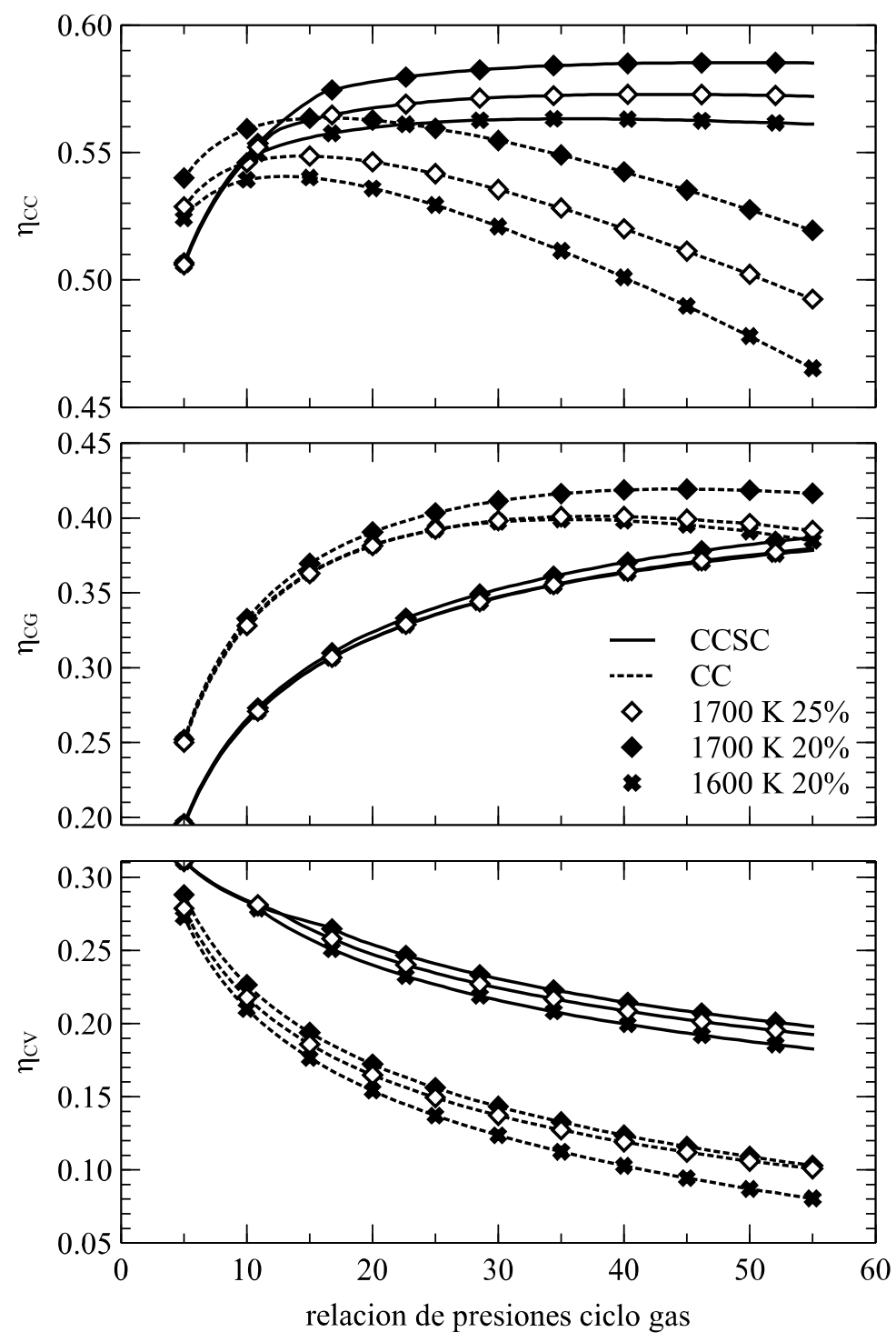

Figura 17. Variación de la eficiencia del ciclo de vapor (inferior), ciclo de gas (medio) y ciclo combinado (superior) con la relación de presiones del ciclo de gas, para distintos valores de $T_{4 t} y x_{\text {refro }}$ para el combinado semicerrado y un ciclo combinado estándar. 
Para explicar las razones de esta diferencia es necesario analizar la descomposición de este rendimiento en el de los ciclos individuales:

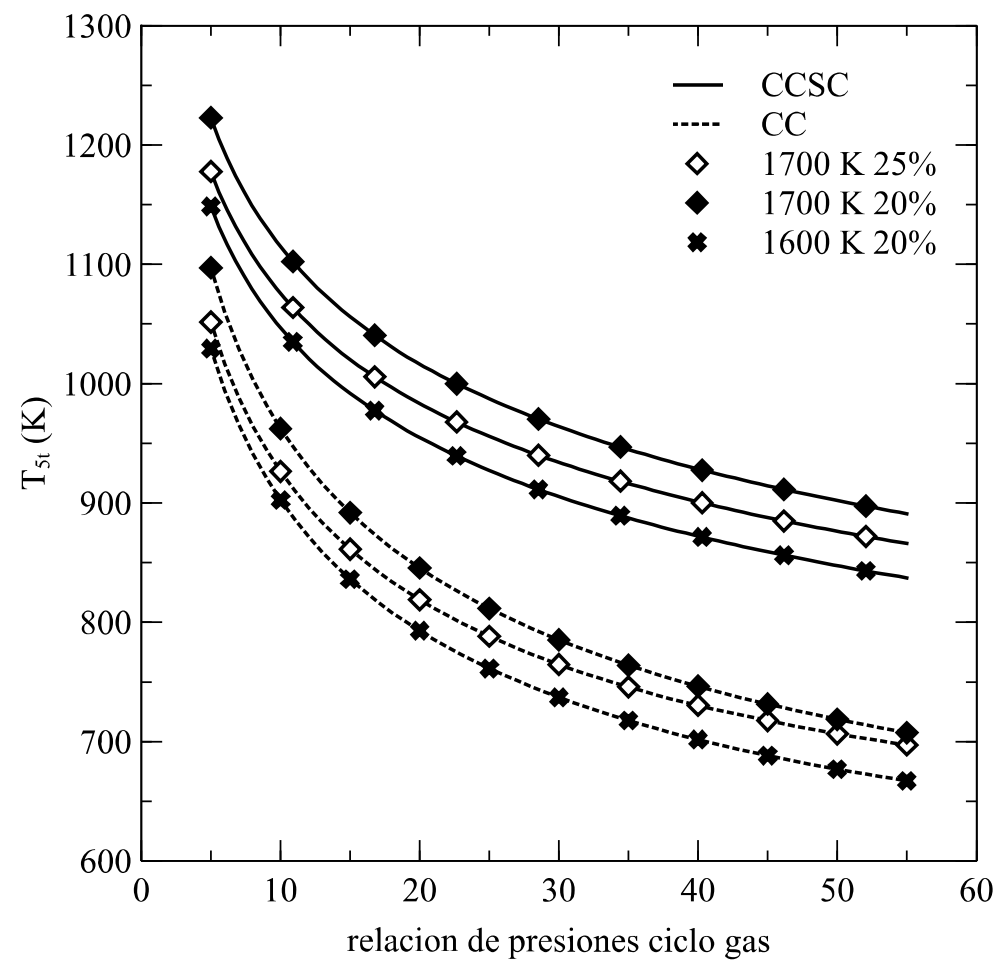

Figura 18. Variación de la temperatura a la salida de la turbina de gas del ciclo combinado semicerrado y el ciclo combinado estándar con la relación de presiones del ciclo de gas, para distintos valores de $T_{4 t} y x_{\text {refr. }}$.

El rendimiento del ciclo de gas se muestra en la parte central de la Figura 17. Tanto para el ciclo con aire como para el ciclo semicerrado, el aumento de $T_{4 t} \sin$ modificar el gasto de refrigeración, caso $1700 \mathrm{~K} 20 \%$, es consistente con el análisis cualitativo del ciclo, aumentando levemente el rendimiento, un 5\% para el ciclo con aire y un 2.4\% para el ciclo semicerrado. En el caso con aire también se desplaza la relación de presiones de rendimiento máximo, desde 35 hasta 44, mientras que en el ciclo semicerrado este punto no varía. Sin embargo, al aumentar el gasto másico de refrigeración la temperatura máxima efectiva del ciclo se reduce, haciendo que el rendimiento del caso $1700 \mathrm{~K} 25 \%$ sea muy similar al del $1600 \mathrm{~K} 20 \%$ tanto para el ciclo con aire como para el ciclo semicerrado. La relación de presiones de máximo rendimiento del caso $1700 \mathrm{~K}$ 
$25 \%$ es ligeramente superior a la del $1600 \mathrm{~K} 25 \%$ para el ciclo con aire, pero casi no cambia para el ciclo semicerrado.

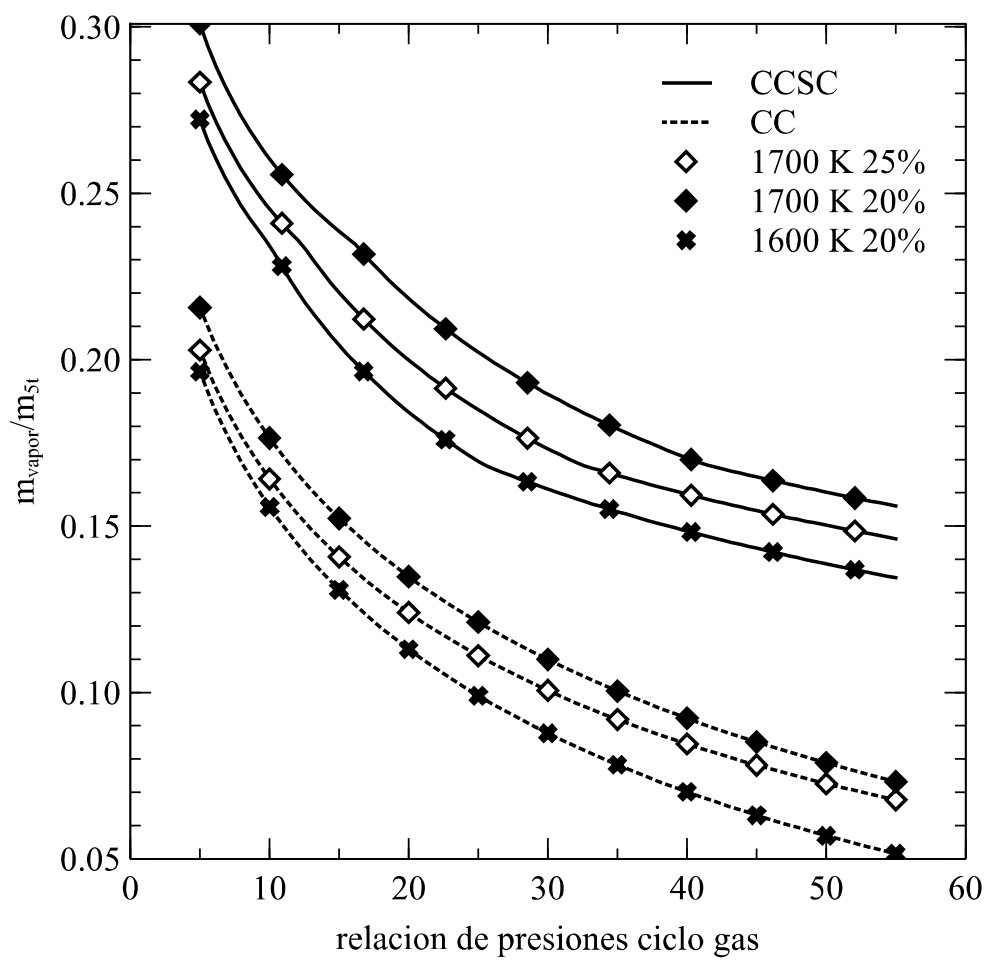

Figura 19. Variación de la relación entre el gasto másico de vapor y el gasto másico de gas a la salida de la turbina del ciclo combinado semicerrado y el ciclo combinado estándar con la relación de presiones del ciclo de gas, para distintos valores de $T_{4 \mathrm{t}} y x_{\text {refr. }}$.

La tendencia de los rendimientos del ciclo de vapor es bastante diferente, como se muestra en la parte inferior de la Figura 17. Para una relación de presiones dada, el aumento de $T_{4 t}$ incrementa el rendimiento del ciclo de vapor, incluso al aumentar el gasto másico de refrigeración. Este aumento está relacionado con el aumento de la temperatura a la salida de la turbina de gas y con el aumento de la relación entre el gasto másico de vapor y el gasto másico del ciclo de gas, que se muestran en la Figura 18 y la Figura 19. En el caso del ciclo semicerrado, para relaciones de presiones bajas se aprecia con claridad el efecto del desplazamiento del punto de mínima diferencia en el generador de vapor, ya comentado al estudiar el efecto de la relación de presiones del ciclo de gas. 

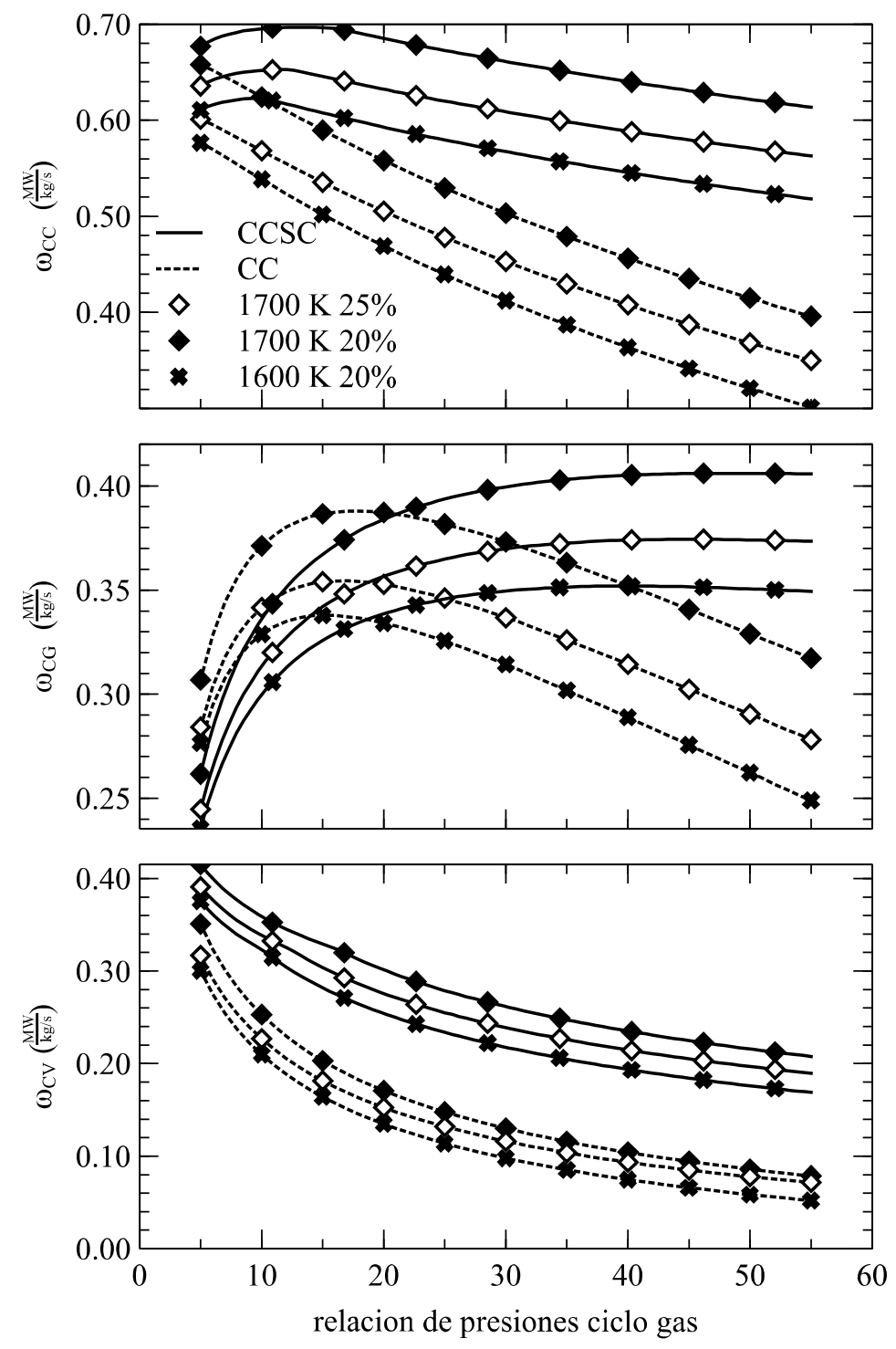

Figura 20. Variación de la potencia específica del ciclo de vapor (inferior), ciclo de gas (medio) y ciclo combinado (superior) con la relación de presiones del ciclo de gas, para distintos valores de $T_{4 t}$ y $x_{\text {refri, }}$ para el ciclo combinado semicerrado y el ciclo combinado estándar.

En la parte central de la Figura 20 se muestra la potencia específica del ciclo de gas, que responde a lo esperado del análisis cualitativo. Tanto en el caso con aire como en el ciclo semicerrado los cambios porcentuales del valor máximo son aproximadamente los mismos que los del ciclo combinado. La relación de presiones del máximo pasa de 15 para el caso $1600 \mathrm{~K} 20 \%$, a 18 para el $1700 \mathrm{~K}$ $20 \%$ y 17 para el 1700K 25\%, en el ciclo con aire, y de 38 para el caso $1600 \mathrm{~K}$ $20 \%$, a 48 para el 1700K 25\% y 45 para el 1700K 20\%. Para los ciclos de gas la distancia entre el caso $1700 \mathrm{~K} 25 \%$ y los otros dos sí se mantiene aproximadamente constante. 
La potencia específica suministrada por el ciclo de vapor se muestra en la parte inferior de la Figura 20. La potencia específica decae con la relación de presiones en todos los casos, siendo mayor para el caso $1700 \mathrm{~K} 20 \%$. El caso $1700 \mathrm{~K} 25 \%$ se mantiene entre el anterior y el caso $1600 \mathrm{~K} 20 \%$, aunque en el ciclo con aire se va acercando al 1700K 20\% para relación de presiones creciente, explicando la tendencia del ciclo combinado.
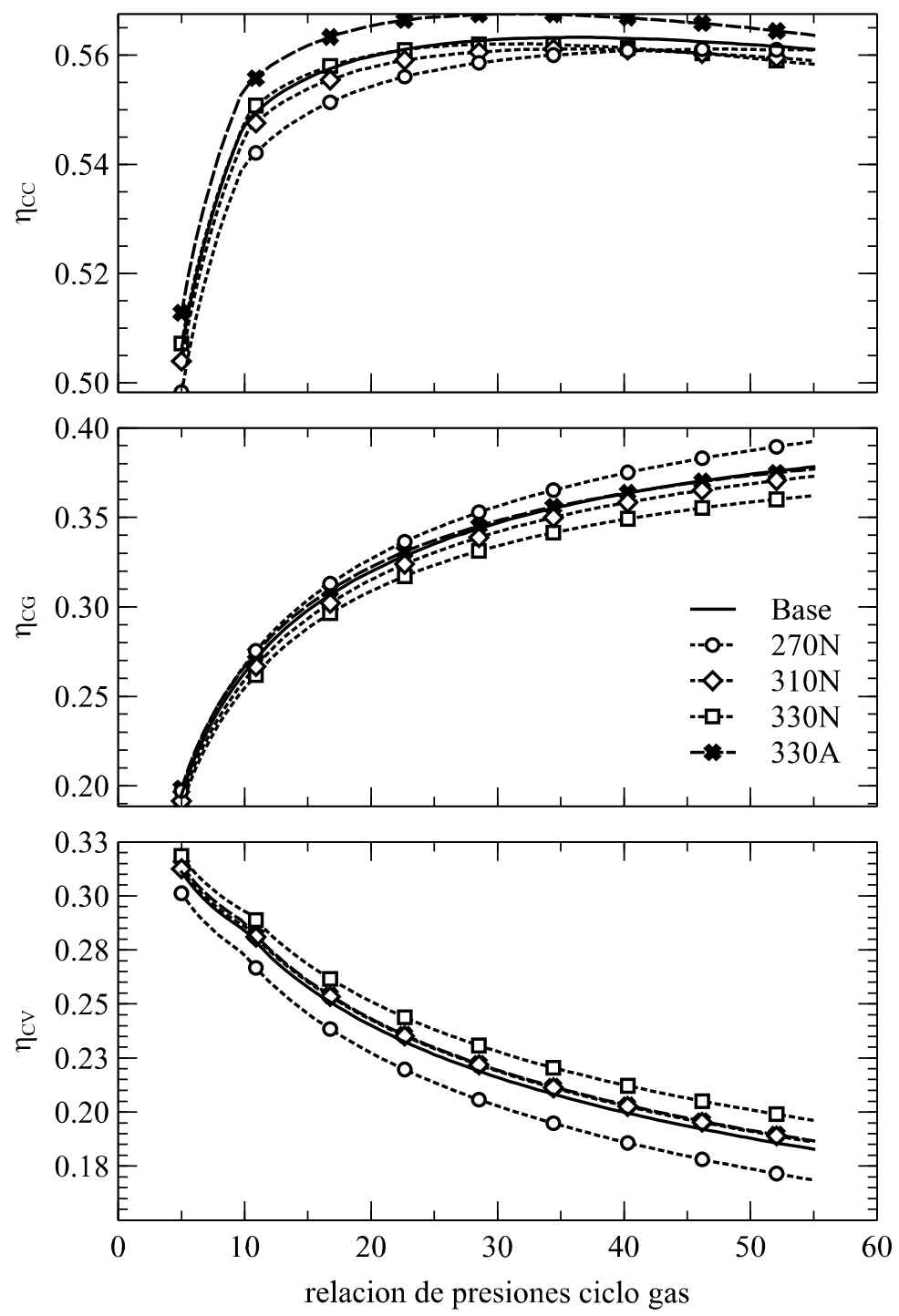

Figura 21. Variación de la eficiencia del ciclo de vapor (inferior), ciclo de gas (medio) y ciclo combinado (superior) con la relación de presiones del ciclo de gas, para distintas temperaturas y composiciones del gas recirculado.

A la vista de los resultados del análisis simplificado, otra forma de intentar mejorar las actuaciones del sistema consiste en enfriar el gas recirculado antes 
de la entrada al compresor. Una forma de conseguirlo sería emplear el gasto másico de $\mathrm{N}_{2}$ que sale de la ASU a baja temperatura. En este sentido, se asume que se puede conseguir una temperatura de $270 \mathrm{~K}$. El efecto de esta disminución de temperatura no se puede separar de los cambios en la composición del gas recirculado. $\mathrm{Al}$ caso con $270 \mathrm{~K}$ se va a asociar un gas recirculado sin presencia de agua, para que sea más sencilla la evaluación del efecto de $T_{2 t}$ también se va a estudiar cómo varían las actuaciones del sistema para este gas sin presencia de agua si se recircula a $310 \mathrm{~K}$ y $330 \mathrm{~K}$. Los casos correspondientes se denominarán 270 N, 310N y 330N. Para completar este estudio de influencia combinada del cambio de temperatura y composición del recirculado se va a incluir un caso a $330 \mathrm{~K}$, elevando el porcentaje de agua hasta el 7.9\%, caso 330A. Todos los resultados se van a comparar con el caso base, con la temperatura y composición de recirculado nominales.

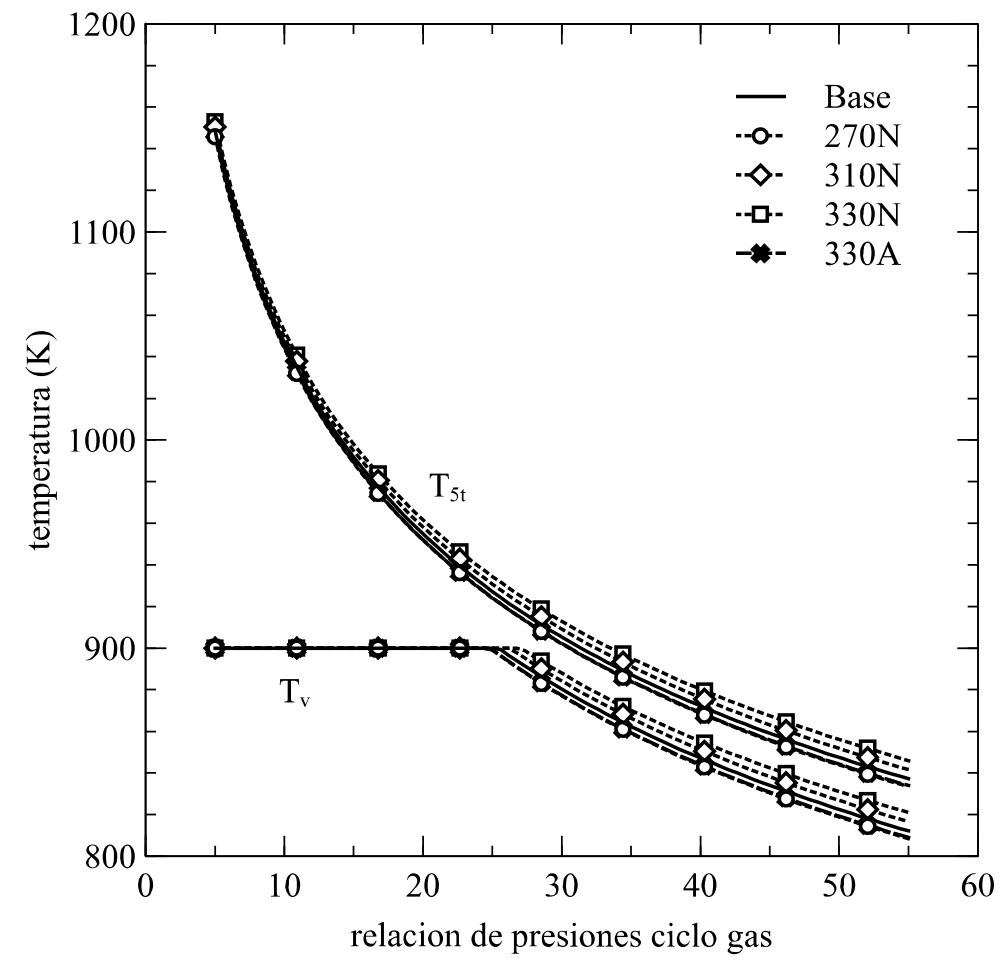

Figura 22. Variación de la temperatura a la salida de la turbina de gas y a la entrada de la turbina de vapor con la relación de presiones del ciclo de gas, para distintas temperaturas y composiciones del gas recirculado.

Los resultados de la turbina de gas, Figura 21 (centro), muestran el incremento esperado de la temperatura para los casos con composición constante $(270 \mathrm{~N}$, 
$310 \mathrm{~N}, 330 \mathrm{~N})$, como se esperaba, mientras que los casos base y 330A proporcionan resultados muy similares.

La eficiencia de la turbina de vapor disminuye, Figura 21 (inferior), debido a la disminución de la entalpía disponible a la salida de la turbina de gas. Esto se debe a que tanto la temperatura de salida de la turbina de gas, Figura 22, como la relación entre el gasto másico de vapor y el de salida de la turbina de gas, Figura 23, disminuyen al disminuir $T_{2 t}$.

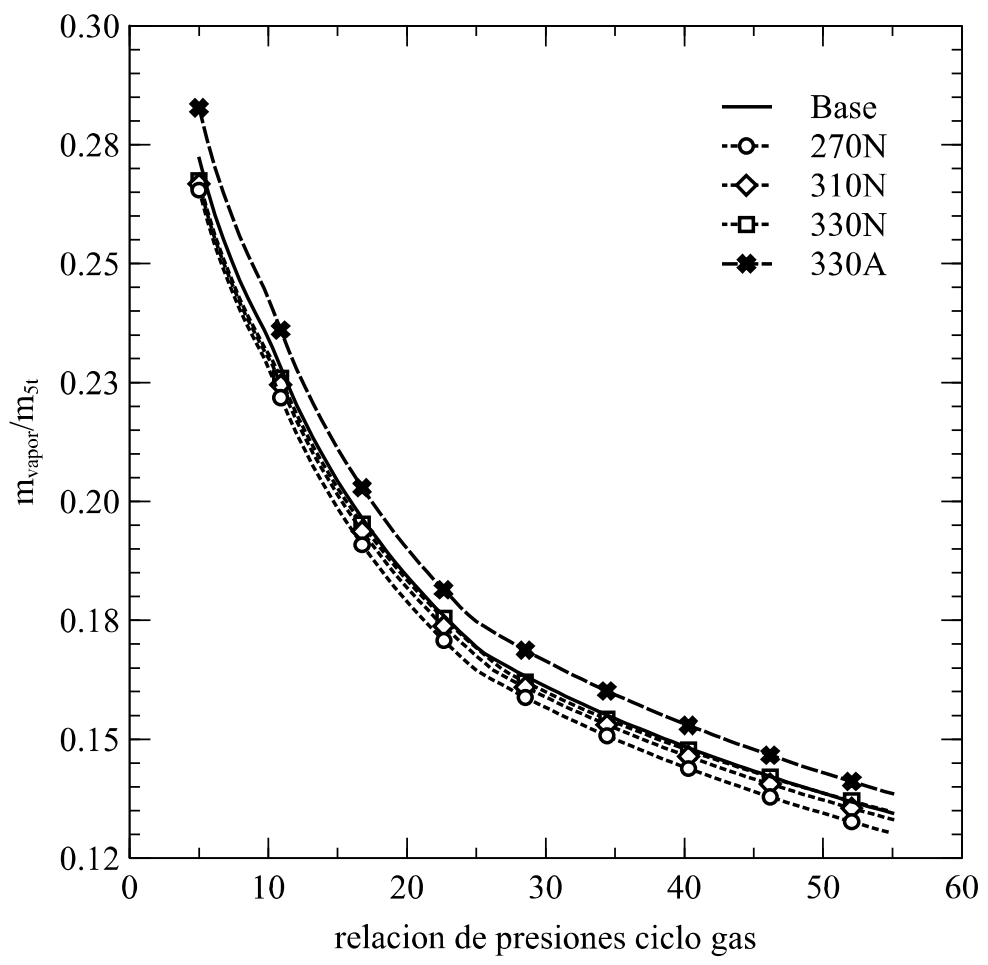

Figura 23. Variación de la relación entre el gasto másico de vapor y el gasto másico de gas a la salida de la turbina del ciclo combinado semicerrado con la relación de presiones del ciclo de gas, para distintas temperaturas y composiciones del gas recirculado.

El efecto del cambio de la temperatura y de la composición del recirculado en la potencia específica es algo más complejo, como puede verse en la Figura 24. Tanto la potencia específica de la turbina de gas como la del ciclo combinado aumentan al disminuir $T_{2 t}$ para composición constante, mientras que el cambio en el ciclo de vapor es relativamente pequeño. Sin embargo, el caso 330A genera una potencia específica del ciclo de gas similar al $270 \mathrm{~N}$ para relaciones de presión bajas, y una potencia específica del ciclo de vapor superior en todo el 
rango, lo que de nuevo puede relacionarse con la mayor relación de gato másico de vapor a gasto másico de gas.
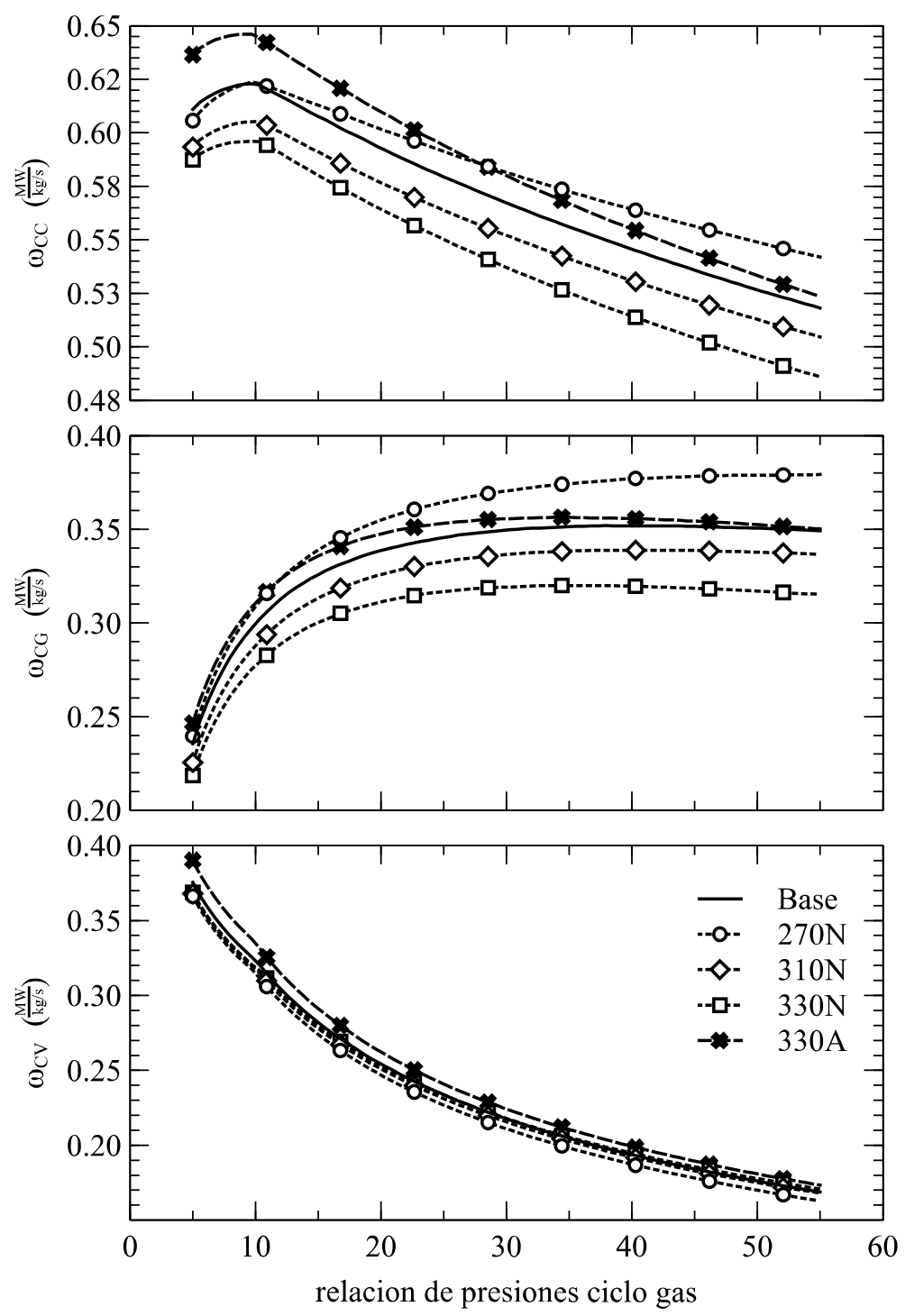

Figura 24. Variación de la potencia específica del ciclo de vapor (inferior), ciclo de gas (medio) y ciclo combinado (superior) con la relación de presiones del ciclo de gas, para distintas temperaturas y composiciones del gas recirculado.

\subsection{Análisis de sensibilidad.}

A partir de los estudios anteriores se ha fijado como punto base del ciclo semicerrado el punto definido por una relación de compresión de 30 y una $T_{4 t}$ de $1600 \mathrm{~K}$, y cuyos resultados globales se presentan en las Tabla 15 a Tabla 18. 
2. Estudio del punto de diseño del ciclo.

\begin{tabular}{cccccccccc}
\hline & gasto & $\mathrm{T}_{\mathrm{t}}$ & $\mathrm{T}_{\mathrm{t}}$ & $\mathrm{P}_{\mathrm{t}}$ & $\mathrm{CO}_{2}$ & $\mathrm{O}_{2}$ & $\mathrm{H}_{2} \mathrm{O}$ & $\mathrm{Ar}$ & $\mathrm{N}_{2}$ \\
Estación & $\mathrm{kg} / \mathrm{s}$ & $\mathrm{K}$ & ${ }^{\circ} \mathrm{C}$ & $\mathrm{kPa}$ & \% masa & \% masa & \% masa & \% masa & \% masa \\
\hline $12 \mathrm{t}\left(\mathrm{O}_{2}\right)$ & 61.5 & 291 & 18 & 125 & 0.00 & 95.00 & 0.00 & 5.00 & 0.00 \\
$13 \mathrm{t}\left(\mathrm{O}_{2}\right)$ & 61.5 & 751 & 478 & 3000 & 0.00 & 95.00 & 0.00 & 5.00 & 0.00 \\
$2 \mathrm{t}\left(\mathrm{CO}_{2}\right)$ & 638.3 & 310 & 37 & 100 & 87.42 & 2.50 & 2.80 & 6.50 & 0.78 \\
$3 \mathrm{t}\left(\mathrm{CO}_{2}\right)$ & 510.6 & 665 & 392 & 3000 & 87.42 & 2.50 & 2.80 & 6.50 & 0.78 \\
$\mathrm{~m}_{\mathrm{NGV}}$ & 85.1 & 665 & 392 & 3000 & 87.42 & 2.50 & 2.80 & 6.50 & 0.78 \\
$\mathrm{M}_{\text {rotor }}$ & 42.6 & 665 & 392 & 3000 & 87.42 & 2.50 & 2.80 & 6.50 & 0.78 \\
$31 \mathrm{t}$ & 572.1 & 674 & 401 & 3000 & 78.02 & 12.44 & 2.50 & 6.34 & 0.70 \\
$4 \mathrm{t}$ & 587.4 & 1600 & 1327 & 2910 & 82.95 & 2.37 & 7.77 & 6.17 & 0.74 \\
$41 \mathrm{t}$ & 672.5 & 1494 & 1221 & 2910 & 83.51 & 2.39 & 7.14 & 6.21 & 0.75 \\
$43 \mathrm{t}$ & 672.5 & 1210 & 937 & 643 & 83.51 & 2.39 & 7.14 & 6.21 & 0.75 \\
$45 \mathrm{t}$ & 715.1 & 1181 & 908 & 643 & 83.75 & 2.39 & 6.88 & 6.23 & 0.75 \\
$5 \mathrm{t}$ & 715.1 & 905 & 632 & 104 & 83.75 & 2.39 & 6.88 & 6.23 & 0.75 \\
combustible & 15.3 & 298 & 25 & & & & & & \\
\hline
\end{tabular}

Tabla 15. Resultados del cálculo del ciclo base ( $\left.P R=30, T_{4 t}=1600 \mathrm{~K}, x_{\text {refr }}=20 \%\right)$, por estaciones.

\begin{tabular}{cccc}
\hline & $\begin{array}{c}\text { Potencia } \\
\mathrm{kW}\end{array}$ & $\begin{array}{c}\mathrm{e}_{\mathrm{p}} \\
\%\end{array}$ & $\begin{array}{c}\eta \\
\%\end{array}$ \\
\hline Compresor $\mathrm{CO}_{2}$ & 224987 & 90.53 & 87.00 \\
Compresor $\mathrm{O}_{2}$ & 27052 & 91.17 & 87.00 \\
Turbina & 254585 & 87.50 & 89.00 \\
Turbina de potencia & 250000 & 86.98 & 89.00 \\
\hline
\end{tabular}

Tabla 16. Resultados del cálculo del ciclo base ( $\left.P R=30, T_{4 t}=1600 \mathrm{~K}, x_{\text {refr }}=20 \%\right)$, para los distintos componentes de la turbomaquinaria.

\begin{tabular}{cccc}
\hline & $\begin{array}{c}\text { gasto } \\
\mathrm{kg} / \mathrm{s}\end{array}$ & $\begin{array}{c}\text { Potencia especifica } \\
\mathrm{kW} /(\mathrm{kg} / \mathrm{s})\end{array}$ & $\begin{array}{c}\text { Potencia } \\
\mathrm{kW}\end{array}$ \\
\hline ASU & 61.5 & 756.0 & 46500 \\
\hline
\end{tabular}

Tabla 17. Consumo de la ASU en el ciclo base $\left(P R=30, T_{4 t}=1600 \mathrm{~K}, x_{\text {refr }}=20 \%\right)$.

\begin{tabular}{cccc}
\hline & $\begin{array}{c}\text { rendimiento } \\
\%\end{array}$ & $\begin{array}{c}\text { potencia especifica } \\
\mathrm{kW} /(\mathrm{kg} / \mathrm{s})\end{array}$ & $\begin{array}{c}\text { potencia } \\
\mathrm{kW}\end{array}$ \\
\hline ciclo gas & 34.69 & 349.6 & 250000 \\
ciclo vapor & 21.60 & 217.7 & 155660 \\
CCSC & 56.29 & 567.3 & 405660 \\
CCSC + ASU + CCS & 47.55 & 479.2 & 342675 \\
\hline
\end{tabular}

Tabla 18. Resultados globales del ciclo base $\left(P R=30, T_{4 t}=1600 \mathrm{~K}, x_{\text {refr }}=20 \%\right)$.

Sobre este punto base se va a considerar el efecto de los parámetros de diseño: la temperatura máxima del ciclo de la turbina de gas, los rendimientos de la fase 
2. Estudio del punto de diseño del ciclo.

de compresión y expansión, y la fracción másica de refrigeración total, del NGV y del rotor. Este estudio es necesario para estimar los cambios que se producirían en el sistema debido a posibles mejoras en la tecnología o a errores cometidos en su selección.

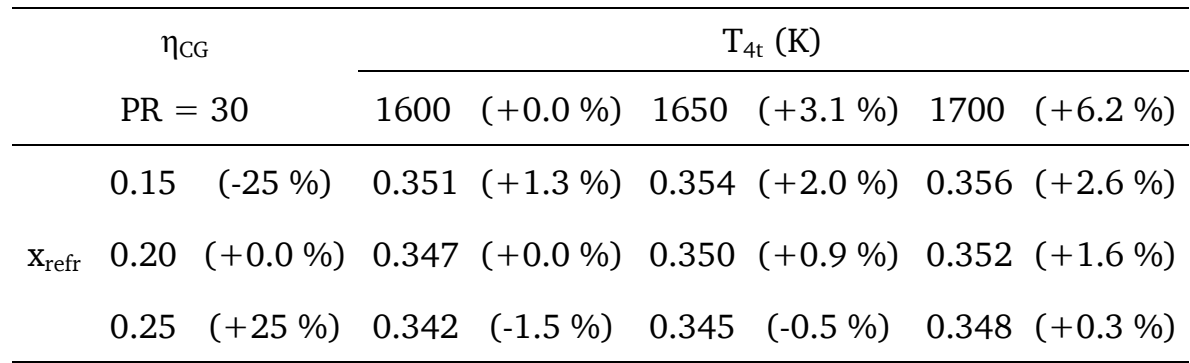

a) Variación del rendimiento de la turbina de gas con $T_{4 t}$ y la fracción másica de gas empleado en la refrigeración, $x_{\text {refr. }}$.

\begin{tabular}{cccccccccc}
\hline \multicolumn{3}{c}{$\eta_{\mathrm{CG}}$} & \multicolumn{6}{c}{$\mathrm{x}_{\mathrm{NGV}}$} \\
\cline { 4 - 9 } $\mathrm{PR}=$ & $30, \mathrm{~T}_{4 \mathrm{t}}=1600 \mathrm{~K}$ & 0.1000 & $(-25 \%)$ & 0.1333 & $(+0.0 \%)$ & 0.1667 & $(+25 \%)$ \\
\hline 0.0500 & $(-25 \%)$ & 0.351 & $(+1.3 \%)$ & 0.350 & $(+0.8 \%)$ & 0.348 & $(+0.3 \%)$ \\
$\mathrm{x}_{\text {Rotor }}$ & 0.0667 & $(+0.0 \%)$ & 0.349 & $(+0.5 \%)$ & 0.347 & $(+0.0 \%)$ & 0.345 & $(-0.6 \%)$ \\
& 0.0833 & $(+25 \%)$ & 0.346 & $(-0.3 \%)$ & 0.344 & $(-0.9 \%)$ & 0.342 & $(-1.5 \%)$ \\
\hline
\end{tabular}

b) Variación del rendimiento de la turbina de gas con la distribución de gasto empleado en la refrigeración.

\begin{tabular}{ccccccccc}
\hline \multicolumn{2}{c}{$\eta_{\mathrm{CG}}$} & \multicolumn{6}{c}{$\eta_{\mathrm{HPC}}$} \\
\cline { 3 - 8 } $\mathrm{PR}=30, \mathrm{~T}_{4 \mathrm{t}}=1600 \mathrm{~K}$ & 0.850 & $(-2.3 \%)$ & 0.870 & $(+0.0 \%)$ & 0.890 & $(+2.3 \%)$ \\
\hline 0.870 & $(-2.2 \%)$ & 0.327 & $(-5.6 \%)$ & 0.333 & $(-4.1 \%)$ & 0.338 & $(-2.6 \%)$ \\
$\eta_{\mathrm{HPT}, \mathrm{PT}}$ & 0.890 & $(+0.0 \%)$ & 0.342 & $(-1.5 \%)$ & 0.347 & $(+0.0 \%)$ & 0.352 & $(+1.4 \%)$ \\
& 0.910 & $(+2.2 \%)$ & 0.356 & $(+2.6 \%)$ & 0.361 & $(+4.0 \%)$ & 0.366 & $(+5.4 \%)$ \\
\hline
\end{tabular}

c) Variación del rendimiento de la turbina de gas con el rendimiento isentrópico de la turbomaquinaria.

Tabla 19. Variación del rendimiento de la turbina de gas con los rendimientos de compresión y expansión, $T_{4 t}$ y fracciones másicas de refrigeración.

En la Tabla 19 se muestra la influencia de las distintas variables en la eficiencia del ciclo de gas:

- La Tabla 19.a muestra el efecto de $T_{4 t}$ junto con la fracción másica de refrigeración global, un efecto ya mostrado con anterioridad combinado 
con el efecto de la relación de presiones. Una subida de 50 K (3.1\%) produce una ganancia del rendimiento próxima al 1\%.

- El efecto del gasto másico de refrigeración y su reparto entre NGV y rotor se muestra en Tabla 19.b. La subida de un 25\% de la fracción másica de refrigeración se convierte en una caída del rendimiento de la turbina de gas de un $1.5 \%$, siendo más sensible a la fracción másica de refrigeración del rotor. Esta mayor sensibilidad es debida a las hipótesis de simulación; se supone que el 50\% se mezcla con la corriente principal a la salida de la turbina y ello es equivalente a una caída de presión importante en esta corriente de refrigeración.

- Los efectos de los rendimientos tanto de la fase de compresión y de la de expansión, tienen una gran influencia en el rendimiento de la turbina de gas, que se muestra en la Tabla 19.c. Una subida del rendimiento de la fase de compresión del $2 \%$ se traduce en una subida del rendimiento de la turbina de gas superior al $1 \%$, mientras que una subida del $2 \%$ en la fase de expansión se traduce prácticamente en el $4 \%$ de subida en el rendimiento de la turbina de gas. La mejora en los rendimientos de compresión y expansión originaría ganancias importantes.

Estos resultados se pueden comparar con los obtenidos para la potencia específica de la turbina de gas, en la Tabla 20:

- En cuanto a la temperatura máxima del ciclo su sensibilidad es apreciablemente superior, como se ve en la Tabla 20.a. Una subida de 50 K (3.1\%) se traduce en una subida de la potencia específica del $7 \%$ aproximadamente, frente al $1 \%$ en el caso del rendimiento de la turbina de gas. Este efecto supone una gran influencia en el tamaño de la turbina de gas.

- La influencia de la fracción másica para refrigeración es claramente mayor; una subida de la fracción másica de refrigeración de un $25 \%$ se traduce en una caída de la potencia específica de un 7\%, en este caso es 
2. Estudio del punto de diseño del ciclo.

mayor el efecto de la fracción másica del NGV, como se ve en la Tabla 20.b.

- Los efectos de los rendimientos de las fases de compresión y expansión también son considerables, con una subida de la influencia del rendimiento de la fase de compresión, como se ve en la Tabla 20.c.

\begin{tabular}{ccccccccc}
\hline \multirow{2}{*}{$\omega_{\mathrm{CG}}(\mathrm{kW} /(\mathrm{kg} / \mathrm{s}))$} & \multicolumn{6}{c}{$\mathrm{T}_{4 \mathrm{t}}(\mathrm{K})$} \\
\cline { 3 - 8 } & $\mathrm{PR}=30$ & 1600 & $(+0.0 \%)$ & 1650 & $(+3.1 \%)$ & 1700 & $(+6.2 \%)$ \\
\hline & 0.15 & $(-25 \%)$ & 374 & $(+6.9 \%)$ & 399 & $(+14.3 \%)$ & 426 & $(+21.8 \%)$ \\
$\mathrm{x}_{\text {refr }}$ & 0.20 & $(+0.0 \%)$ & 350 & $(+0.0 \%)$ & 374 & $(+7.1 \%)$ & 399 & $(+14.3 \%)$ \\
& 0.25 & $(+25 \%)$ & 325 & $(-7.1 \%)$ & 349 & $(-0.2 \%)$ & 373 & $(+6.7 \%)$ \\
\hline
\end{tabular}

a) Variación de la potencia específica de la turbina de gas con $T_{4 t}$ y la fracción másica de gas empleado en la refrigeración, $x_{\text {refr. }}$.

\begin{tabular}{ccccccccc}
\hline \multirow{2}{*}{$\omega_{\mathrm{CG}}(\mathrm{kW} /(\mathrm{kg} / \mathrm{s}))$} & \multicolumn{6}{c}{$\mathrm{x}_{\mathrm{NGV}}$} \\
\cline { 3 - 9 } $\mathrm{PR}=$ & $30, \mathrm{~T}_{4 \mathrm{t}}=1600 \mathrm{~K}$ & 0.1000 & $(-25 \%)$ & 0.1333 & $(+0.0 \%)$ & 0.1667 & $(+25 \%)$ \\
\hline & 0.0500 & $(-25 \%)$ & 374 & $(+6.9 \%)$ & 359 & $(+2.7 \%)$ & 344 & $(-1.6 \%)$ \\
$\mathrm{x}_{\text {Rotor }}$ & 0.0667 & $(+0.0 \%)$ & 364 & $(+4.3 \%)$ & 350 & $(+0.0 \%)$ & 334 & $(-4.3 \%)$ \\
& 0.0833 & $(+25 \%)$ & 355 & $(+1.6 \%)$ & 340 & $(-2.7 \%)$ & 325 & $(-7.1 \%)$ \\
\hline
\end{tabular}

b) Variación de la potencia específica de la turbina de gas con la distribución de gasto empleado en la refrigeración.

\begin{tabular}{rrrrrrrrr}
\hline \multirow{2}{*}{$\omega_{\mathrm{CG}}(\mathrm{kW} /(\mathrm{kg} / \mathrm{s}))$} & \multicolumn{6}{c}{$\eta_{\mathrm{HPC}}$} \\
\cline { 3 - 9 } $\mathrm{PR}=30, \mathrm{~T}_{4 \mathrm{t}}=1600 \mathrm{~K}$ & 0.850 & $(-2.3 \%)$ & 0.870 & $(+0.0 \%)$ & 0.890 & $(+2.3 \%)$ \\
\hline 0.870 & $(-2.2 \%)$ & 328 & $(-6.2 \%)$ & 335 & $(-4.1 \%)$ & 342 & $(-2.0 \%)$ \\
$\eta_{\mathrm{HPT}, \mathrm{PT}}$ & 0.890 & $(+0.0 \%)$ & 342 & $(-2.1 \%)$ & 350 & $(+0.0 \%)$ & 357 & $(+2.0 \%)$ \\
& 0.910 & $(+2.2 \%)$ & 356 & $(+1.9 \%)$ & 364 & $(+4.0 \%)$ & 371 & $(+6.0 \%)$ \\
\hline
\end{tabular}

c) Variación de la potencia específica de la turbina de gas con el rendimiento isentrópico de la turbomaquinaria.

Tabla 20. Variación de la potencia específica de la turbina de gas con los rendimientos de compresión y expansión, $T_{4 t}$ y fracciones másicas de refrigeración.

Por su relevancia para el ciclo de vapor, también se muestra la variación de la temperatura de salida de la turbina, en la Tabla 21. 
2. Estudio del punto de diseño del ciclo.

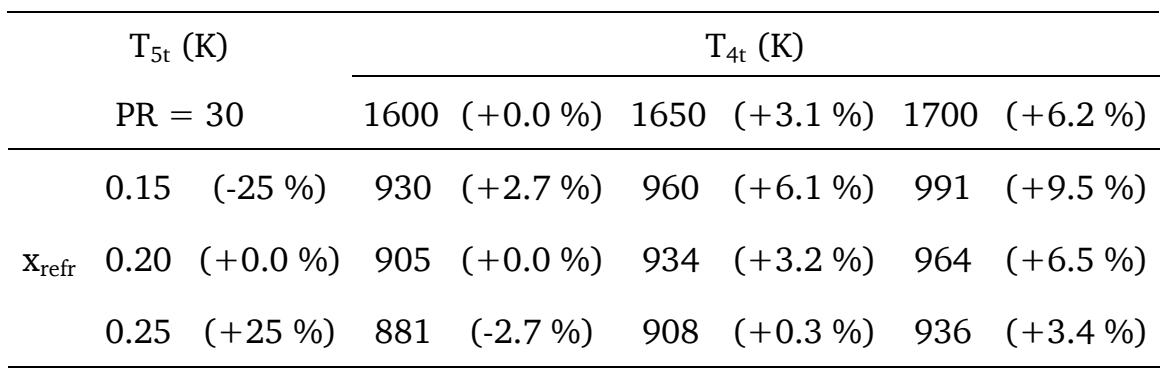

a) Variación de $T_{5 t}$ con $T_{4 t} y$ la fracción másica de gas empleado en la refrigeración, $x_{\text {refr. }}$.

\begin{tabular}{cccccccccc}
\hline $\mathrm{T}_{5 \mathrm{t}}(\mathrm{K})$ & \multicolumn{6}{c}{$\mathrm{x}_{\mathrm{NGV}}$} \\
\cline { 3 - 8 } $\mathrm{PR}=$ & $30, \mathrm{~T}_{4 \mathrm{t}}=1600 \mathrm{~K}$ & 0.1000 & $(-25 \%)$ & 0.1333 & $(+0.0 \%)$ & 0.1667 & $(+25 \%)$ \\
\hline & 0.0500 & $(-25 \%)$ & 930 & $(+2.7 \%)$ & 913 & $(+0.8 \%)$ & 895 & $(-1.1 \%)$ \\
$\mathrm{x}_{\text {Rotor }}$ & 0.0667 & $(+0.0 \%)$ & 923 & $(+1.9 \%)$ & 905 & $(+0.0 \%)$ & 888 & $(-1.9 \%)$ \\
& 0.0833 & $(+25 \%)$ & 916 & $(+1.1 \%)$ & 898 & $(-0.8 \%)$ & 881 & $(-2.7 \%)$ \\
\hline
\end{tabular}

b) Variación de $T_{5 t}$ con la distribución de gasto empleado en la refrigeración.

\begin{tabular}{|c|c|c|c|c|c|c|c|c|}
\hline \multirow{2}{*}{\multicolumn{3}{|c|}{$\begin{array}{c}\mathrm{T}_{5 \mathrm{t}}(\mathrm{K}) \\
\mathrm{PR}=30, \mathrm{~T}_{4 \mathrm{t}}=1600 \mathrm{~K}\end{array}$}} & \multicolumn{6}{|c|}{$\eta_{\mathrm{HPC}}$} \\
\hline & & & 0.850 & $(-2.3 \%)$ & 0.870 & $(+0.0 \%)$ & 0.890 & $(+2.3 \%)$ \\
\hline \multirow{3}{*}{$\eta_{\mathrm{HPT}, \mathrm{PT}}$} & 0.870 & $(-2.2 \%)$ & 918 & $(+1.4 \%)$ & 917 & $(+1.3 \%)$ & 916 & $(+1.2 \%)$ \\
\hline & 0.890 & $(+0.0 \%)$ & 906 & $(+0.1 \%)$ & 905 & $(+0.0 \%)$ & 905 & $(-0.1 \%)$ \\
\hline & 0.910 & $(+2.2 \%)$ & 895 & $(-1.2 \%)$ & 894 & $(-1.3 \%)$ & 893 & $(-1.3 \%)$ \\
\hline
\end{tabular}

c) Variación de $T_{5 t}$ con el rendimiento isentrópico de la turbomaquinaria, respecto al caso base.

Tabla 21. Variación de la temperatura de salida de la turbina de gas, $T_{5 t}$, con los rendimientos de compresión y expansión, $T_{4 t}$ y fracciones másicas de refrigeración.

- Como se ve en la Tabla 21.a, la temperatura de salida es muy sensible a variaciones en $T_{4 t}$. Una subida del 3\% (3.1\%) aproximadamente se traduce en aproximadamente el mismo porcentaje de subida de temperatura de salida de la turbina de potencia (3.2\%).

- En cuanto a la fracción másica para refrigeración su sensibilidad es inferior a la existente en el caso de la potencia específica; un $25 \%$ se transforma en un 3\% aproximadamente, siendo de nuevo mayor el efecto de la fracción másica del NGV, como se ve en la Tabla 21.b.

- Finalmente la influencia de los rendimientos de expansión y compresión, Tabla 21.c, es inferior a la obtenida en los casos del rendimiento de la turbina de gas y de la potencia específica. La influencia del rendimiento 
de compresión es muy pequeña, mientras que un $2 \%$ de subida en el rendimiento de expansión se traduce en una subida de la temperatura de salida de aproximadamente un $1 \%$.

\begin{tabular}{|c|c|c|c|c|c|c|c|}
\hline & & & & & $4 \mathrm{t}(\mathrm{K})$ & & \\
\hline & $\mathrm{PR}=30$ & 1600 & $(+0.0 \%)$ & 1650 & $(+3.1 \%)$ & 1700 & $(+6.2 \%)$ \\
\hline & $(-25 \%)$ & 0.573 & $(+1.8 \%)$ & 0.583 & $(+3.6 \%)$ & 0.592 & $(+5.1 \%)$ \\
\hline $\mathrm{x}_{\text {refr }}$ & $0.20(+0.0 \%)$ & 0.563 & $(+0.0 \%)$ & 0.574 & $(+1.9 \%)$ & 0.583 & $(+3.6 \%)$ \\
\hline & $(+25 \%)$ & 0.551 & $(-2.1 \%)$ & 0.563 & $(+0.0 \%)$ & 0.573 & $(+1.8 \%)$ \\
\hline
\end{tabular}

a) Variación del rendimiento del ciclo combinado semicerrado con $T_{4 t}$ y la fracción másica de gas empleado en la refrigeración, $x_{\text {refr. }}$.

\begin{tabular}{cccccccccc}
\hline \multicolumn{3}{c}{$\eta_{\mathrm{CG}}$} & \multicolumn{6}{c}{$\mathrm{x}_{\mathrm{NGV}}$} \\
\cline { 3 - 9 } $\mathrm{PR}=$ & $30, \mathrm{~T}_{4 \mathrm{t}}=1600 \mathrm{~K}$ & 0.1000 & $(-25 \%)$ & 0.1333 & $(+0.0 \%)$ & 0.1667 & $(+25 \%)$ \\
\hline 0.0500 & $(-25 \%)$ & 0.573 & $(+1.8 \%)$ & 0.567 & $(+0.7 \%)$ & 0.560 & $(-0.5 \%)$ \\
$\mathrm{x}_{\text {Rotor }}$ & 0.0667 & $(+0.0 \%)$ & 0.570 & $(+1.2 \%)$ & 0.563 & $(+0.0 \%)$ & 0.556 & $(-1.3 \%)$ \\
& 0.0833 & $(+25 \%)$ & 0.566 & $(+0.5 \%)$ & 0.559 & $(-0.8 \%)$ & 0.551 & $(-2.1 \%)$ \\
\hline
\end{tabular}

b) Variación del rendimiento del ciclo combinado semicerrado con la distribución de gasto empleado en la refrigeración.

\begin{tabular}{rrrrrrrrr}
\hline & & \multicolumn{6}{c}{$\eta_{\mathrm{HPC}}$} \\
\cline { 3 - 8 } $\mathrm{PR}=30, \mathrm{~T}_{4 \mathrm{t}}=1600 \mathrm{~K}$ & 0.850 & $(-2.3 \%)$ & 0.870 & $(+0.0 \%)$ & 0.890 & $(+2.3 \%)$ \\
\hline 0.870 & $(-2.2 \%)$ & 0.554 & $(-1.6 \%)$ & 0.557 & $(-1.0 \%)$ & 0.560 & $(-0.5 \%)$ \\
$\eta_{\mathrm{HPT}, \mathrm{PT}}$ & 0.890 & $(+0.0 \%)$ & 0.560 & $(-0.6 \%)$ & 0.563 & $(+0.0 \%)$ & 0.566 & $(-0.5 \%)$ \\
& 0.910 & $(+2.2 \%)$ & 0.566 & $(+0.5 \%)$ & 0.569 & $(+1.0 \%)$ & 0.572 & $(-1.5 \%)$ \\
\hline
\end{tabular}

c) Variación del rendimiento del ciclo combinado semicerrado con el rendimiento isentrópico de la turbomaquinaria, respecto al caso base.

Tabla 22. Variación del rendimiento del ciclo combinado semicerrado con los rendimientos de compresión y expansión, $T_{4 t}$ y fracciones másicas de refrigeración.

Aunque se ha presentado la influencia de la variación de algunos parámetros en algunas variables de interés de la turbina de gas, resulta de mayor interés el efecto sobre el ciclo combinado. La Tabla 22 muestra el rendimiento del sistema completo, sin tener en cuenta la ASU ni el CCS. Se puede ver que es muy sensible tanto a la temperatura máxima del ciclo (Tabla 22.a) como a los 
rendimientos de las fases de expansión y compresión (Tabla 22.c). Un 3\% de subida de la temperatura máxima del ciclo se traduce en una subida similar del rendimiento del sistema ( $2 \%$ ), mientras una subida del $2 \%$ en el rendimiento de expansión se traduce aproximadamente en una subida del 1\%; la influencia del rendimiento de compresión es inferior, un $2 \%$ de subida en dicho rendimiento se traduce en un $0.5 \%$ en el rendimiento global del sistema.

El tercer parámetro por su influencia en el rendimiento global, está representado por la fracción másica de refrigeración. Su subida en un $25 \%$ se traduce en una bajada del rendimiento global inferior al 1\%. En el rendimiento global de nuevo es mayor el efecto de la fracción másica de refrigeración del NGV (Tabla 22.b). 
2. Estudio del punto de diseño del ciclo. 


\section{ESTUDIO DEL CICLO A CARGA PARCIAL.}

En esta sección se pretende obtener la configuración del ciclo fuera de su punto de diseño. Para ello se va a utilizar de nuevo un modelo de simulación basada en componentes, aunque los modelos de los componentes y del sistema son diferentes. Estos modelos presentan las mismas ventajas de gran velocidad de cálculo y de no requerir la especificación de un elevado número de parámetros. La flexibilidad asociada a estos modelos también permite la simulación y comparación de distintas configuraciones de ejes y leyes de control, entre las que se pretende seleccionar la más apropiada. Un problema asociado al uso de estos modelos es que será necesario obtener mapas de comportamiento apropiados para la turbomaquinaria, mediante la adaptación de mapas de turbomaquinaria funcionando con aire.

Se van a comparar distintas leyes de control similares a las usadas en las turbinas de gas de ciclos combinados convencionales junto a una ley de control que se usa en ciclos cerrados. Para simplificar la comparación, en esta sección solo se van a presentar resultados del ciclo combinado semicerrado, sin compararlo con ciclos combinados convencionales.

En primer lugar se presentará el comportamiento del ciclo para cada ley de control, procediendo después a su comparación, centrada en el rendimiento del sistema completo. De nuevo, se espera que este análisis aproximado sirva como base para un estudio posterior más detallado de los componentes.

\subsection{Modelo de simulación.}

Para la simulación del sistema se va a emplear el método de simulación basada en componentes, aunque con ciertas diferencias debido al cambio de modo de funcionamiento. Los cálculos se llevarán a cabo con el programa SFSA y el modelo de composición de equilibrio de los gases en cada estación. 


\section{Estudio del ciclo a carga parcial.}

Se asume que el comportamiento de la ASU no cambia respecto al de diseño, pudiendo modelarse como un consumo proporcional al gasto másico de oxidante generado. El compresor del oxidante generado por la ASU se comporta de manera similar al modo de diseño, especificando su rendimiento isentrópico. Aunque es de esperar que ambos componentes vean su comportamiento modificado a carga parcial, se espera que dichos cambios sean despreciables frente a los cambios en el resto del ciclo.

Las condiciones del gas recirculado podrán cambiar respecto a las del punto de diseño, dependiendo del método de control utilizado. Este aspecto se comentará en detalle más adelante.

El compresor de gas recirculado se ha modelado utilizando mapas, como es usual en condiciones fuera de su punto de diseño, aunque hay que tener en cuenta dos consideraciones adicionales:

- Los compresores para turbinas de gas de generación de potencia disponen de álabes guía de geometría variable a la entrada, VIGV (Variable Inlet Guide Vanes), así como de varias etapas de estatores de geometría variable, VSV, (Variable Stator Vanes). Esto permite controlar el gasto que pasa por el compresor y, por lo tanto, su uso como variable de control en la turbina. Su efecto se va a modelar de una forma muy simplificada, como una pérdida de presión de remanso antes del compresor. Aunque este método produce una eficiencia menor que otros métodos más detallados [47], tiene la ventaja de no necesitar mapas de actuaciones para cada posición del VIGV, VSV [48], curvas características, velocidades y ángulos representativos de cada etapa [49], o coeficientes empíricos adicionales para las correcciones [19].

- Los mapas empleados para simular la turbomaquinaria tienen una gran influencia en el comportamiento del sistema a carga parcial [50]. Sin embargo, no hay disponible en la literatura abierta ningún mapa de un compresor axial de $\mathrm{CO}_{2}$ a la escala requerida. Aunque ya se han empleado 
modelos muy simplificados con anterioridad, como líneas de vueltas corregidas con gasto corregido constante [22]-[25] o diseños simplificados de compresores [2]-[4], en este caso se ha optado por adaptar el mapa de un compresor de una turbina de producción de potencia convencional al funcionamiento con el gas recirculado, escalándolo mediante un método que se describirá posteriormente.

El gasto másico de combustible inyectado en la cámara se va a utilizar como variable de control de la turbina, permitiendo regular la potencia producida en conjunción con las demás variables de control disponibles. La relación entre los gastos másicos de combustible y de la ASU se mantendrá igual al valor especificado para el punto de diseño.

Como en el estudio del punto de diseño del ciclo, se va a suponer la existencia de una turbina de alta, que suministra potencia al compresor y al resto de los subsistemas, y una turbina de potencia que genera la potencia neta del ciclo de gas. El comportamiento de ambas se va a modelar mediante mapas, teniendo en cuenta dos posibles configuraciones:

- Las turbinas aeroderivadas, $\mathrm{AD}$, cuentan con dos ejes. El compresor y la turbina de alta se encuentran en el mismo eje, mientras que la turbina de potencia funciona como turbina libre acoplada al generador eléctrico, y su velocidad de giro suele suponerse constante, con independencia del resto del ciclo. Este tipo de turbinas se modelará suponiendo que las vueltas de la HPT y las de la PT pueden variar de manera independiente.

- Las turbinas monoeje o tipo Heavy Duty, HD, tienen un solo eje en el que están acoplados el compresor y la turbina, que también estará acoplada al generador. Este tipo de turbinas se modelará imponiendo que la HPT y la PT giren a la misma velocidad.

Los gastos másicos de refrigeración se mantendrán iguales a los especificados en el punto de diseño. 
El comportamiento a carga parcial del ciclo de vapor se va a simular permitiendo que cambie la presión máxima del ciclo, con temperatura constante del condensador, para adaptarse a las condiciones del ciclo de gas, modelo habitual en la literatura [51].

El calor transferido entre el ciclo de gas y el ciclo de vapor en el generador de vapor se calculará mediante el método NTU- $\varepsilon$ [52] para las áreas calculadas en el punto de diseño.

\subsection{Obtención de los mapas de compresor y turbinas.}

El análisis dimensional del flujo a través de una turbomáquina permite reducir el número de variables independientes mediante la formación de grupos adimensionales. Por ejemplo, para un compresor, el estado del flujo en la estación de salida 3t, que se suele representar mediante la temperatura de remanso, $T_{3 t}$ y la presión de remanso, $P_{3 t}$, se asume que es función de:

- Las condiciones del flujo a la entrada, representadas por su temperatura de remanso, $T_{2 t}$, y su presión de remanso, $P_{2 t}$,

- Las condiciones de operación del compresor, representadas por el flujo másico a la entrada, $m$, y la velocidad de giro, $N$,

- La geometría de la máquina, representada por una longitud característica cono su diámetro, $D$.

- Y las propiedades del fluido, en este caso la constante del gas, $R$, la capacidad térmica específica a presión constante, $c_{p}$, la conductividad térmica, $k$, y la viscosidad, $\mu$. Por simplicidad se asume que estas propiedades no varían en el proceso.

Con estas condiciones, el cambio en el estado del gas a través del compresor se puede expresar en la forma: 


$$
\begin{aligned}
& P_{3 t}=f_{1}\left(T_{2 t}, P_{2 t}, m, N, D, R, c_{p}, \mu, k\right) \\
& T_{3 t}=f_{2}\left(T_{2 t}, P_{2 t}, m, N, D, R, c_{p}, \mu, k\right)
\end{aligned}
$$

Como solo hay cuatro dimensiones básicas diferentes (masa, longitud, tiempo y temperatura), estos nueve parámetros dimensionales se pueden reducir a cinco parámetros adimensionales. Es habitual usar $T_{2 t}, P_{2 t}, R$ y $D$ como parámetros para el escalado, proporcionando la relación de capacidades específicas, $\gamma$, el número de Reynolds, Re, y el número de Prandtl, Pr, para las propiedades del gas.

La selección de los otros grupos presenta más variaciones. El gasto másico y la velocidad de giro pueden adimensionalizarse utilizando los grupos $\sqrt{\gamma R T_{2 t}} / D^{2} P_{2 t} \mathrm{y} D / \sqrt{\gamma R T_{2 t}}$, respectivamente, obteniendo las dependencias:

$$
\begin{aligned}
& \frac{P_{3 t}}{P_{2 t}}=F_{1}\left(\frac{m \sqrt{\gamma R T_{2 t}}}{D^{2} P_{2 t}}, \frac{D N}{\sqrt{\gamma R T_{2 t}}}, \gamma, R_{e}, P_{r}\right) \\
& \frac{T_{3 t}}{T_{2 t}}=F_{2}\left(\frac{m \sqrt{\gamma R T_{2 t}}}{D^{2} P_{2 t}}, \frac{D N}{\sqrt{\gamma R T_{2 t}}}, \gamma, R_{e}, P_{r}\right)
\end{aligned}
$$

Como $\gamma$ es un parámetro adimensional, cuya dependencia sigue apareciendo de forma explícita en las expresiones, no es imprescindible incluirlo en los grupos utilizados para adimensionalizar $m$ y $N$, mientras se conserve su efecto.

Sin embargo, es una práctica habitual despreciar el efecto de los cambios de las propiedades del fluido en el comportamiento del compresor, y asumir que el número de Reynolds es lo suficientemente grande como para despreciar también su efecto y el del número de Prandtl. Como el mapa se genera para una máquina de un tamaño dado, este también se suele eliminar de las expresiones, dando lugar a las funciones de grupos con dimensiones: 


$$
\begin{aligned}
& \frac{P_{3 t}}{P_{2 t}} \simeq F_{1}\left(\frac{m \sqrt{T_{2 t}}}{P_{2 t}}, \frac{N}{\sqrt{T_{2 t}}}\right) \\
& \frac{T_{3 t}}{T_{2 t}} \simeq F_{2}\left(\frac{m \sqrt{T_{2 t}}}{P_{2 t}}, \frac{N}{\sqrt{T_{2 t}}}\right)
\end{aligned}
$$

Además, la temperatura y la presión suelen adimensionalizarse con los valores estándar, $T_{S T D}=288.15 \mathrm{~K}, P_{S T D}=101325 \mathrm{~Pa}$, en la forma:

$$
\begin{gathered}
\delta_{2 t}=\frac{P_{2 t}}{P_{S T D}}, \\
\theta_{2 t}=\frac{T_{2 t}}{T_{S T D}},
\end{gathered}
$$

de modo que, en lugar de tener parámetros adimensionales para el gasto y las vueltas, se tienen un gasto corregido y unas vueltas corregidas con las mismas dimensiones que las originales. Para finalizar la transformación de estas expresiones, la relación $T_{3 t} / T_{2 t}$ se suele fijar mediante el rendimiento isentrópico, $\eta_{23}$, dando lugar a las expresiones:

$$
\begin{aligned}
& \frac{P_{3 t}}{P_{2 t}} \simeq F_{1}\left(\frac{m \sqrt{\theta_{2 t}}}{\delta_{2 t}}, \frac{N}{\sqrt{\theta_{2 t}}}\right) \\
& \eta_{23} \simeq F_{2}\left(\frac{m \sqrt{\theta_{2 t}}}{\delta_{2 t}}, \frac{N}{\sqrt{\theta_{2 t}}}\right)
\end{aligned}
$$

Sin embargo, distintos autores emplean diferentes variantes de este método, llegando a otras expresiones y mapas.

Para estudiar las actuaciones a carga parcial del ciclo semicerrado funcionando con un gas rico en $\mathrm{CO}_{2}$ se va a obtener un mapa representativo de las actuaciones de la turbomaquinaria a partir de mapas que se presentan en la forma simplificada anterior. Como la dependencia con las propiedades del fluido ha sido eliminada, es necesario recuperarla, aunque sea de una manera aproximada. 
Para ello se van a aplicar técnicas de análisis dimensional desarrolladas para estimar el efecto de la humedad del aire en las actuaciones de la turbomaquinaria [53]. Estas técnicas se basan en la elección de ciertos parámetros de similaridad que conservan un sentido físico determinante del comportamiento del fluido a través de la turbomáquina. En este estudio solo se van a presentar los parámetros utilizados, remitiendo al lector a la referencia original para los orígenes de cada transformación. Los parámetros de similaridad escogidos son:

1.- El gasto másico a la entrada dividido entre el gasto másico crítico a la entrada. La igualdad de este parámetro llevaría a números de Mach axiales muy parecidos. Usando este parámetro, la relación entre los gastos másicos corregidos puede expresarse en la forma:

$$
\left(\frac{m \sqrt{\theta_{2 t}}}{\delta_{2 t}}\right)_{R}=\sqrt{\frac{R_{\text {aire }}}{R_{R}}} \sqrt{\frac{\gamma_{R}}{\gamma_{\text {aire }}}} \frac{\left(\frac{\gamma_{\text {aire }}+1}{2}\right)^{\frac{\gamma_{\text {aire }}+1}{2\left(\gamma_{\text {are }}-1\right)}}}{\left(\frac{\gamma_{R}+1}{2}\right)^{\frac{\gamma_{R}+1}{\left.2 \gamma_{R}-1\right)}}}\left(\frac{m \sqrt{\theta_{2 t}}}{\delta_{2 t}}\right)_{\text {aire }},
$$

donde el subíndice $R$ representa al gas recirculado.

2.- El parámetro de vueltas $N / \sqrt{\gamma R T_{2 t}}$. La igualdad de este parámetro lleva a números de Mach circunferenciales muy parecidos. Esta condición puede escribirse en la forma:

$$
\left(\frac{N}{\sqrt{\theta_{2 t}}}\right)_{R}=\frac{\sqrt{\gamma_{R} R_{R}}}{\sqrt{\gamma_{\text {aire }} R_{\text {aire }}}}\left(\frac{N}{\sqrt{\theta_{2 t}}}\right)_{\text {aire }}
$$

La conservación de los números de Mach axial y circunferencial lleva a la igualdad del coeficiente de carga $\Delta h_{23} / \gamma R T_{2 t}$. Esta condición puede escribirse en la forma: 


$$
\left(\frac{T_{3 t}}{T_{2 t}}\right)_{R}=1+\frac{\gamma_{R}-1}{\gamma_{\text {aire }}-1}\left(\left(\frac{T_{3 t}}{T_{2 t}}\right)_{\text {aire }}-1\right)
$$

Los cambios en la eficiencia se van a estimar asumiendo que se mantiene el rendimiento politrópico. Este método se ha comparado con otras correlaciones [54], proporcionando resultados parecidos sin la necesidad de fijar parámetros adicionales. La condición referida puede expresarse en la forma:

$$
\left(\frac{P_{3 t}}{P_{2 t}}\right)_{R}=\left[1+\frac{\gamma_{R}-1}{\gamma_{\text {aire }}-1}\left(\left(\frac{P_{3 t}}{P_{2 t}}\right)_{\text {aire }}^{\frac{\gamma_{\text {aire }}-1}{e_{\text {aire }}}}-1\right)\right]^{\frac{e_{R} \gamma_{R}}{\gamma_{R}-1}},
$$

donde $e_{\text {aire }} \mathrm{y} e_{R}$ representan el rendimiento politrópico del proceso con aire y con gas recirculado, respectivamente.

\begin{tabular}{ccccc}
\hline & $\begin{array}{c}\mathrm{m} \sqrt{ } \theta / \delta \\
\mathrm{kg} / \mathrm{s}\end{array}$ & $\mathrm{P}_{3 \mathrm{t}} / \mathrm{P}_{2 \mathrm{t}}$ & $\begin{array}{c}\eta \\
\%\end{array}$ & $\begin{array}{c}\mathrm{N} / \sqrt{ } \theta \\
\%\end{array}$ \\
\hline LM 2500 & 65.03 & 18 & 86.2 & 100 \\
$\mathrm{LM} \mathrm{2500+}$ & 84.46 & 23.6 & 86.6 & 100 \\
$\mathrm{LM} \mathrm{2500+} \mathrm{R}$ & 98.14 & 25.9 & 87.6 & 80.1 \\
\hline
\end{tabular}

Tabla 23. Transformación del punto de diseño del mapa del compresor de la turbina LM 2500.

El mapa que se va a emplear en este estudio es el mapa del compresor del motor CF6, usado en la turbina aeroderivada de producción de potencia LM2500 [55]. Este se puede a escalar usando técnicas estándar [48], para obtener un mapa representativo del compresor de la turbina de gas LM2500+ [40]. A partir de este mapa, se ha aplicado la transformación anteriormente explicada a las condiciones del gas recirculado, obteniendo la transformación del punto de diseño representada en la Tabla 23.

El efecto predominante, tanto en el gasto corregido como en las vueltas corregidas, se debe a la relación entre las constantes del gas, mientras que el cambio en el rendimiento isentrópico es pequeño. La transformación predice una 
relación de presiones algo mayor para la operación con gas recirculado. Estas tendencias se mantienen en todo el mapa, como puede verse en la Figura 25.
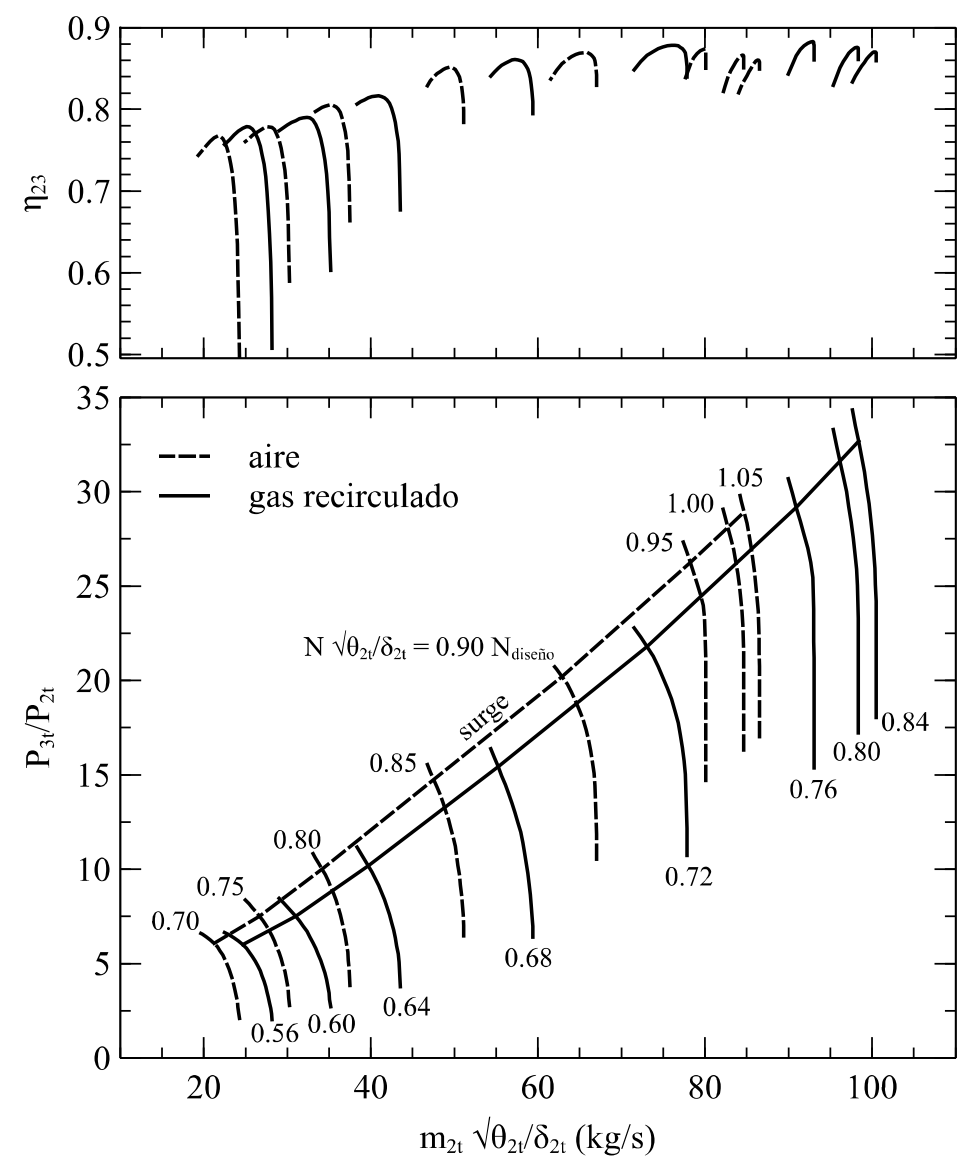

Figura 25. Comparación entre el mapa de la relación de presiones (inferior) y eficiencia (superior) obtenidos para el compresor de la turbina LM2500+ y los obtenidos para el funcionamiento con gas recirculado.

Como el mapa transformado no corresponde a las condiciones de diseño del CCSC, es necesario escalarlo a su vez para obtener el mapa a usar en las simulaciones. El mapa final obtenido se ha representado en la Figura 26.

También es de interés la aplicación de las condiciones de similaridad inversas al punto de diseño del CCSC, para obtener un punto equivalente de funcionamiento con aire. De este modo puede estimarse cómo sería el compresor funcionando con aire más cercano al requerido para el ciclo semicerrado. Los dos puntos de diseño se muestran en la Tabla 24, donde puede verse que las 
condiciones del compresor similar funcionando con aire están dentro del rango de la tecnología actual.

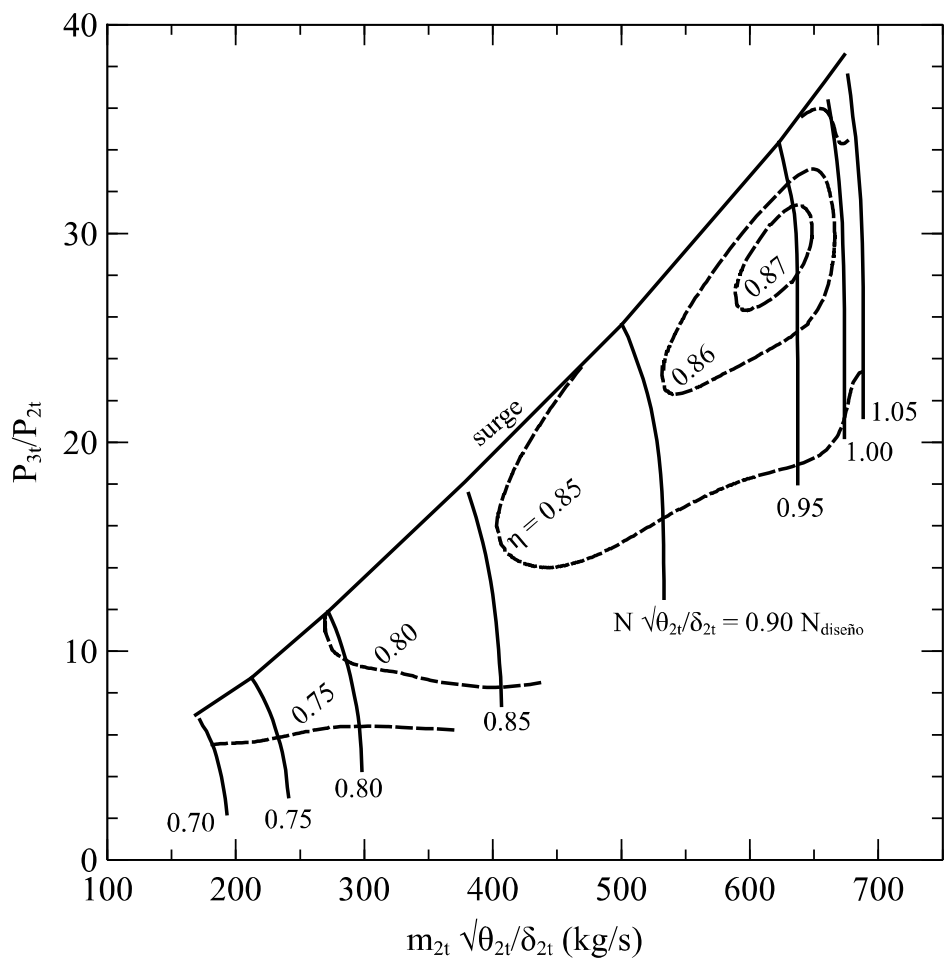

Figura 26. Mapa obtenido para el compresor del ciclo semicerrado.

\begin{tabular}{ccccccc}
\hline & $\begin{array}{c}\mathrm{m} \sqrt{ } \theta / \delta \\
\mathrm{kg} / \mathrm{s}\end{array}$ & $\mathrm{P}_{3 \mathrm{t}} / \mathrm{P}_{2 \mathrm{t}}$ & $\begin{array}{c}\eta \\
\%\end{array}$ & $\begin{array}{c}\mathrm{N} / \\
\%\end{array}$ & $\begin{array}{c}\mathrm{N} \\
\mathrm{rpm}\end{array}$ & $\begin{array}{c}\mathrm{T}_{2 \mathrm{t}} \\
\mathrm{K}\end{array}$ \\
\hline $\mathrm{R}$ & 671.53 & 30 & 87 & 100 & 3000 & 310 \\
aire & 577.93 & 27 & 86 & 125 & 3607 & 288 \\
\hline
\end{tabular}

Tabla 24. Punto de diseño del compresor del CCSC y punto equivalente funcionando con aire aplicando las condiciones de similaridad.

En el caso de la turbina se ha considerado que su comportamiento está fijado por el parámetro de gasto máximo que admite, como es usual en los estudios fuera del punto de diseño. Por ello se ha escalado directamente un mapa genérico, obteniendo el mapa de la Figura 27. Un procedimiento similar permite obtener el mapa de la turbina de potencia. 


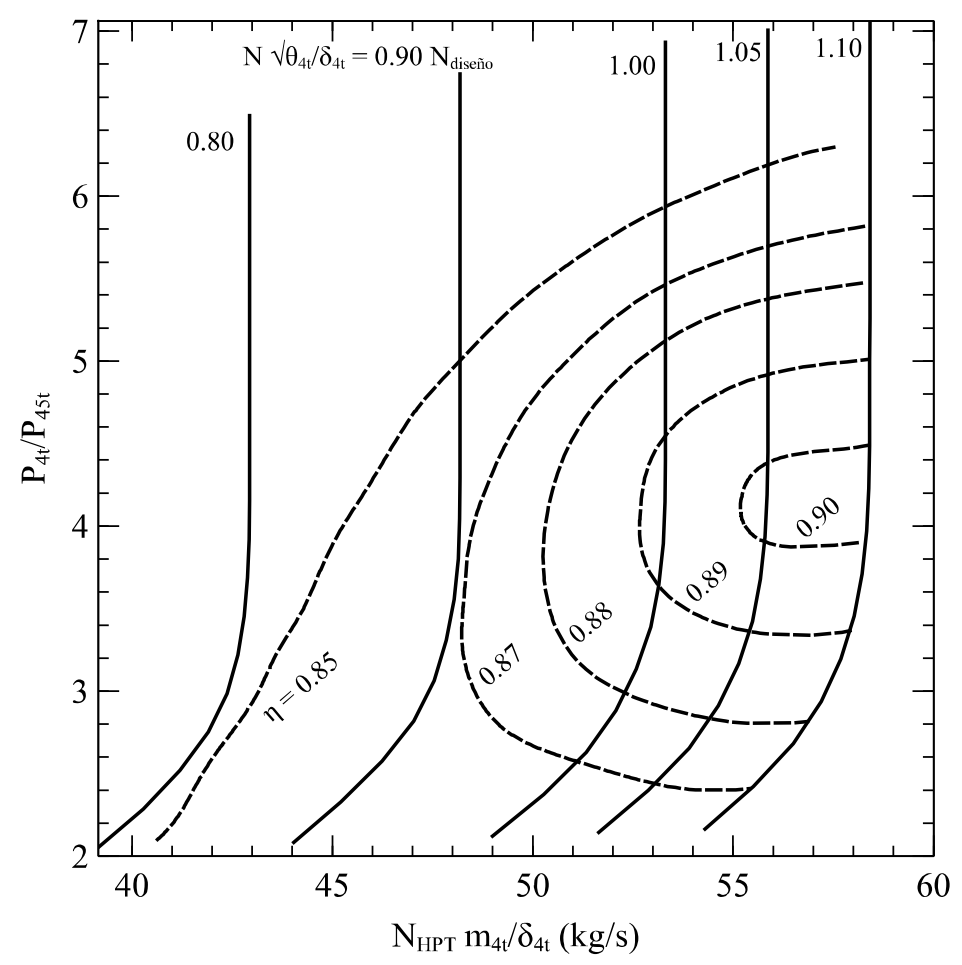

Figura 27. Mapa obtenido para la turbina de alta del ciclo semicerrado.

\subsection{Leyes de control para turbinas aeroderivadas.}

En esta configuración se va a suponer que las condiciones de presión y temperatura del gas recirculado se mantienen iguales a las de diseño. Por lo tanto, se puede contar con dos variables de control, la cantidad de combustible inyectado y el control de gasto del compresor (VIGV/VSV). Al variar estos dos parámetros se puede ajustar la potencia de salida del ciclo de gas y un parámetro adicional. Sin embargo, hay que tener en cuenta que el control VIGV/VSV puede ser válido únicamente hasta una reducción del gasto másico del 30-40\% [16], [19], lo que puede requerir el uso de leyes de control mixtas. Se supondrá que la turbina de potencia permanece a vueltas constantes. 


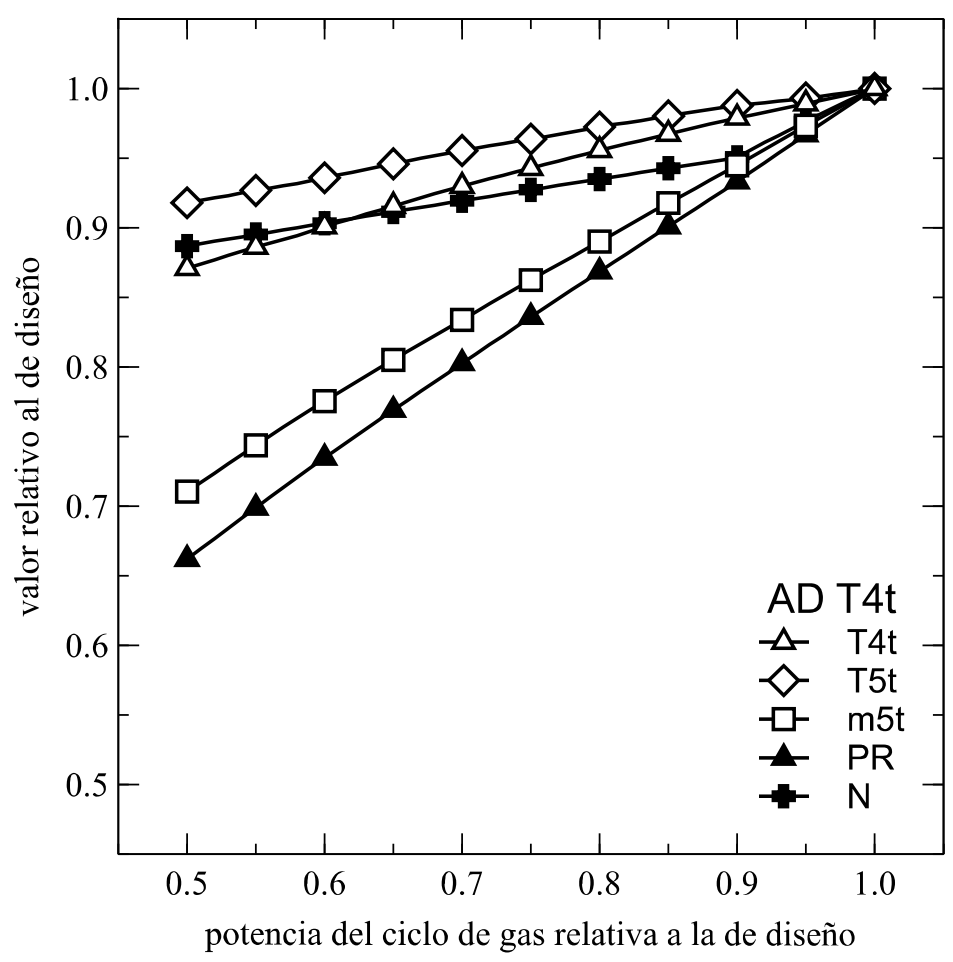

Figura 28. Comportamiento a carga parcial de las variables fundamentales de comportamiento del ciclo semicerrado para turbina aeroderivada con control en $T_{4 t}$.

Teniendo en cuenta que el control de gasto implica la introducción de pérdidas, una ley de control sencilla consiste en asumir que dicho control siempre está abierto, con lo que el gasto de combustible se modifica para variar $T_{4 t} \mathrm{y}$, en consecuencia, la potencia suministrada por la turbina, a esta ley de control se la va a denominar $\mathrm{AD}$ T4t. El comportamiento a carga parcial de las variables fundamentales de comportamiento del ciclo semicerrado, $P R, T_{4 t}, T_{5 t}, m_{5 t} \mathrm{y} N$, para esta ley de control se muestran en la Figura 28. Puede apreciarse que tanto $T_{5 t}$ como $m_{5 t}$ disminuyen al disminuir la potencia, lo que puede influir en la potencia generada en el ciclo de vapor. 


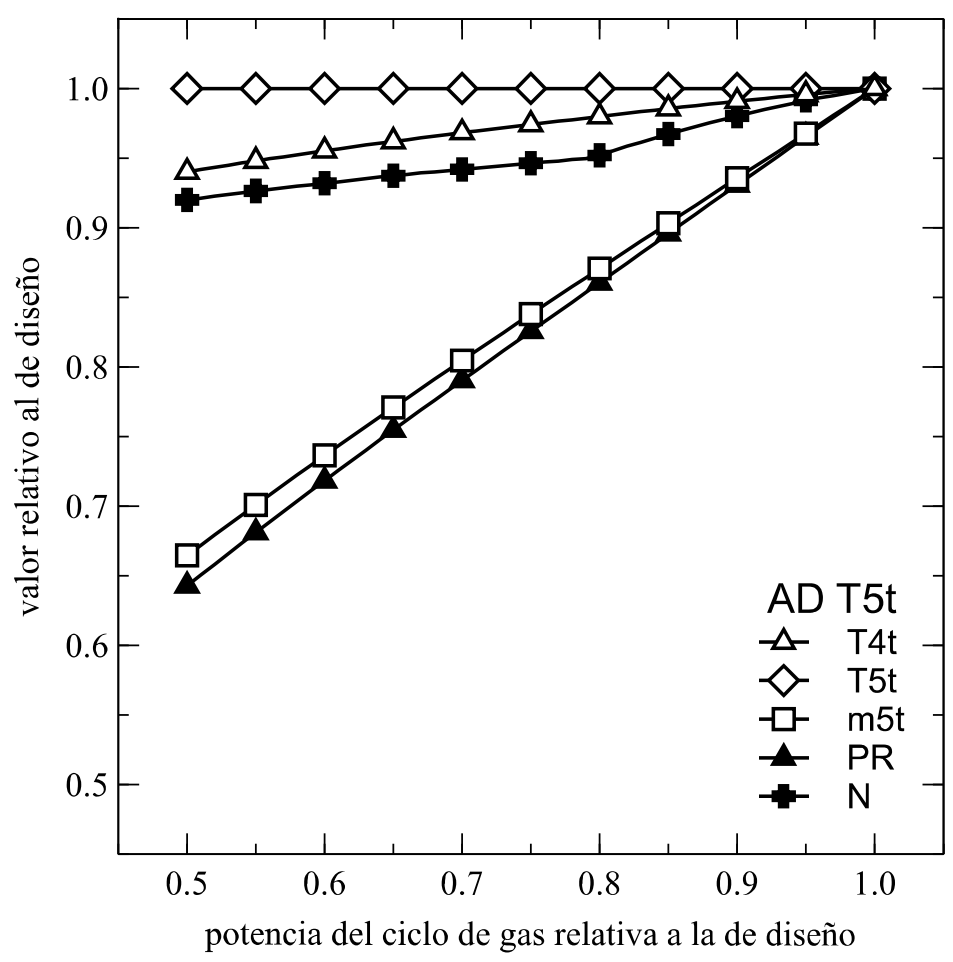

Figura 29. Comportamiento a carga parcial de las variables fundamentales de comportamiento del ciclo semicerrado para turbina aeroderivada con $T_{5 t}$ constante.

Una ley de control alternativa consiste en ajustar las dos variables de control para reducir la potencia, a la vez que se mantiene constante la temperatura de salida de la turbina de potencia, intentando no cambiar las condiciones de trabajo del ciclo de vapor. Esta es una condición de trabajo común en la literatura [16], [19], [21]. El comportamiento del ciclo semicerrado con esta ley, denominada AD T5t, se muestra en la Figura 29, donde puede verse que, además de mantenerse $T_{5 t}$ constante, se consigue una variación lineal con la potencia de $m_{5 t}$.

Teniendo en cuenta que el control VIGV/VSV puede estar limitado, puede ser necesario utilizar leyes de control mixtas. La Figura 30 muestra los resultados a carga parcial del ciclo semicerrado para una ley denominada AD MIX, que consiste en el uso de la ley de control con $T_{5 t}$ constante para potencias entre el $80 \%$ y el $100 \%$, mientras que para potencias inferiores al $80 \%$ se deja constante la posición del control VIGV/VSV y se controla únicamente $T_{4 t}$, mediante el gasto másico de combustible. 


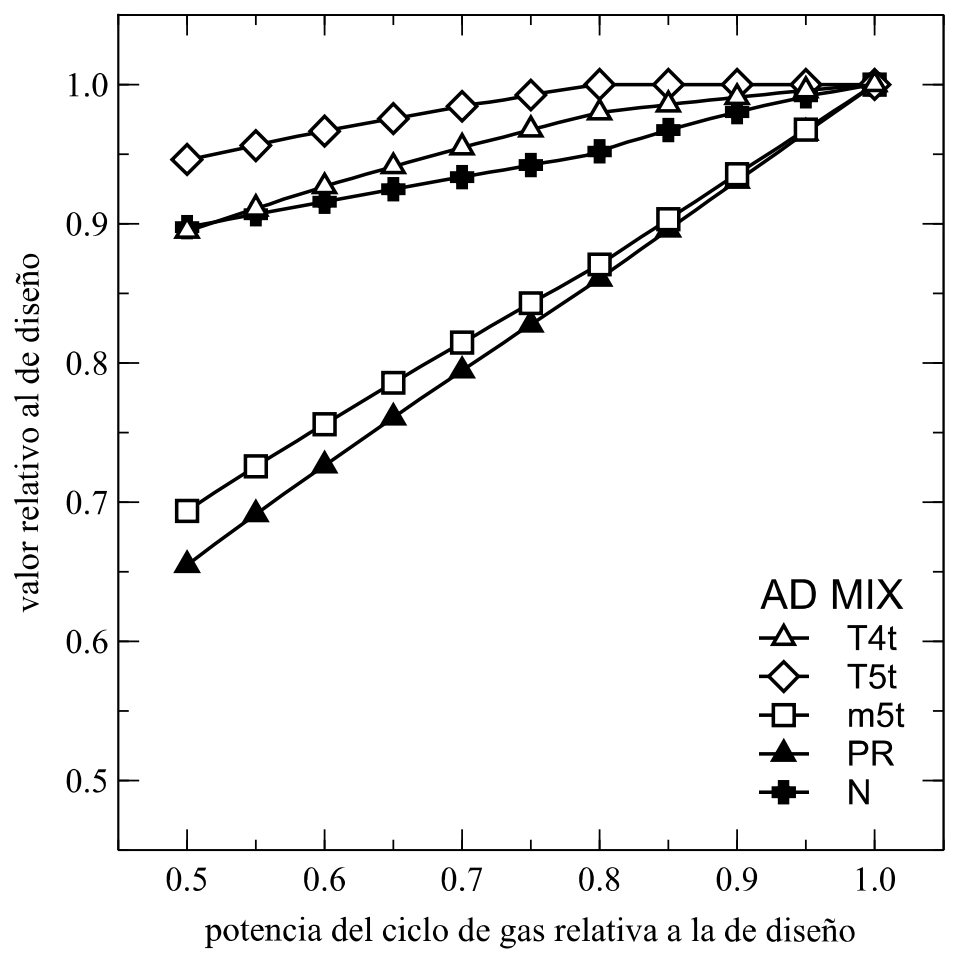

Figura 30. Comportamiento a carga parcial de las variables fundamentales de comportamiento del ciclo semicerrado para turbina aeroderivada con ley de control mixta, $T_{5 t}$ constante entre $100 \%$ y $80 \%$ de carga, seguida de control en $T_{4 t}$.

\subsection{Leyes de control para turbinas monoeje.}

En este caso se va a asumir de nuevo que las condiciones de presión y temperatura del gas recirculado permanecen constantes, dejando el caso en que cambian para más adelante.

Las turbinas monoeje suelen estar acopladas a un generador eléctrico síncrono, con lo que es deseable que funcionen a velocidad constante. Para ello es necesario utilizar los dos grados de libertad disponibles para ajustar la potencia y las vueltas del eje. Los resultados del ciclo semicerrado para esta ley de control, denominada HD N, se representan en la Figura 31. Puede verse que la aplicación de esta ley conduce a una disminución lineal de $m_{5 t}$, junto a un aumento de $T_{5}$, a medida que disminuye la potencia. Este aumento de $T_{5 t}$ podría conducir a problemas en la turbina del ciclo de vapor. También hay que recordar que el control en VIGV/VSV puede no permitir una reducción suficiente del gasto másico en todo el rango de carga parcial. 


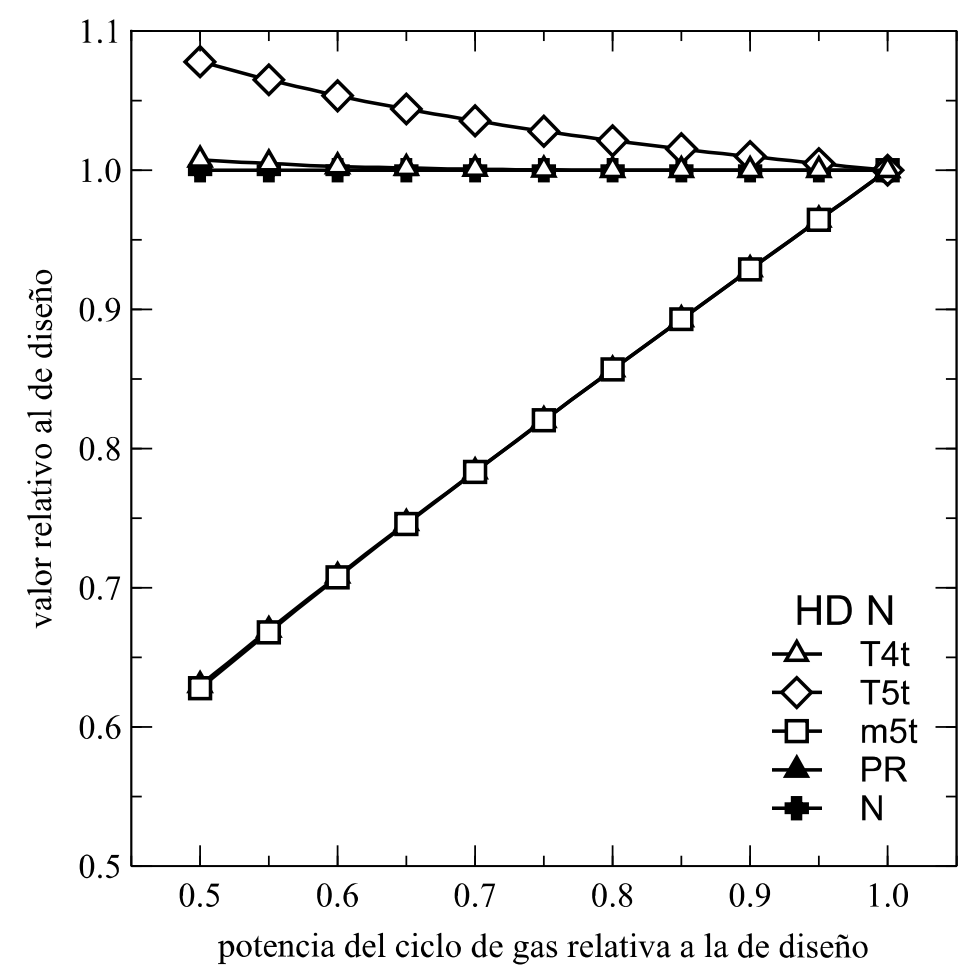

Figura 31. Comportamiento a carga parcial de las variables fundamentales de comportamiento del ciclo semicerrado para turbina monoeje con vueltas constantes.

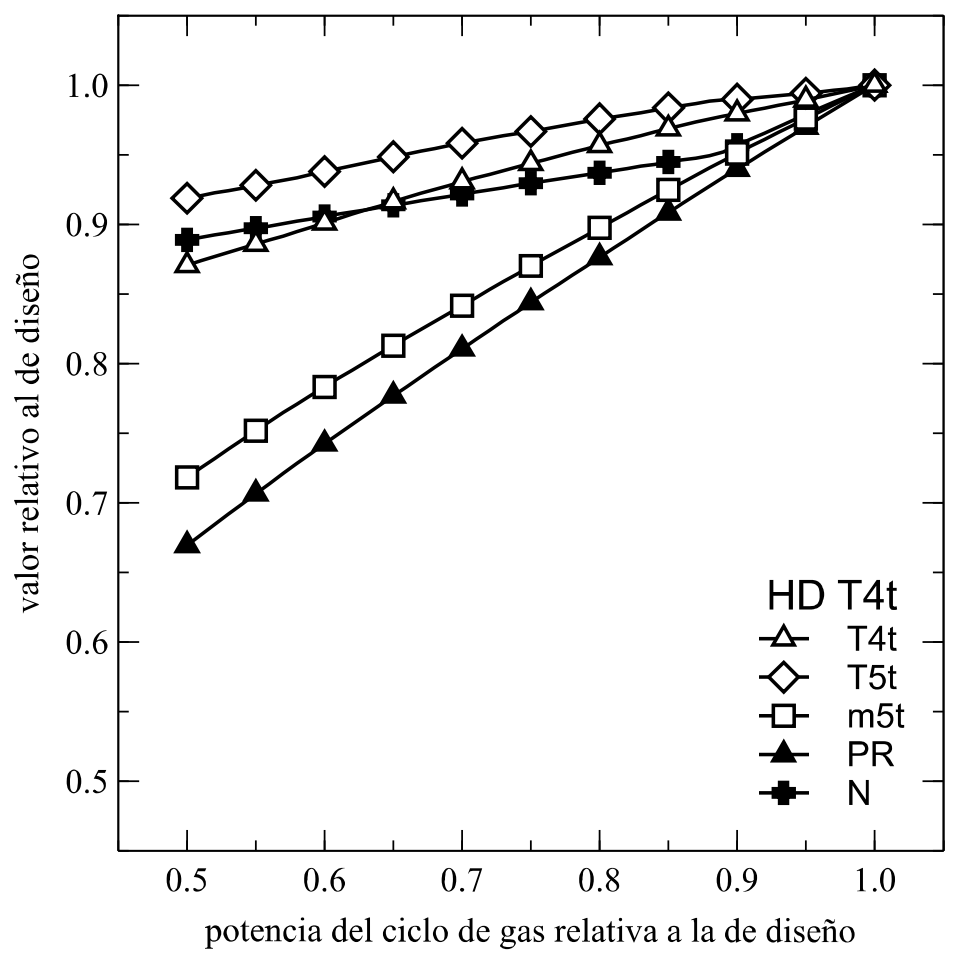

Figura 32. Comportamiento a carga parcial de las variables fundamentales de comportamiento del ciclo semicerrado para turbina monoeje con control en $T_{4 t}$. 
La ley alternativa, ignorando el comportamiento de las vueltas del eje, consiste en variar únicamente $T_{4 t}$, ley denominada HD T4t. El comportamiento del ciclo semicerrado para esta ley se muestra en la Figura 32, done se ve que $N, T_{5 t}$ y $m_{5 t}$ decaen al disminuir la potencia.

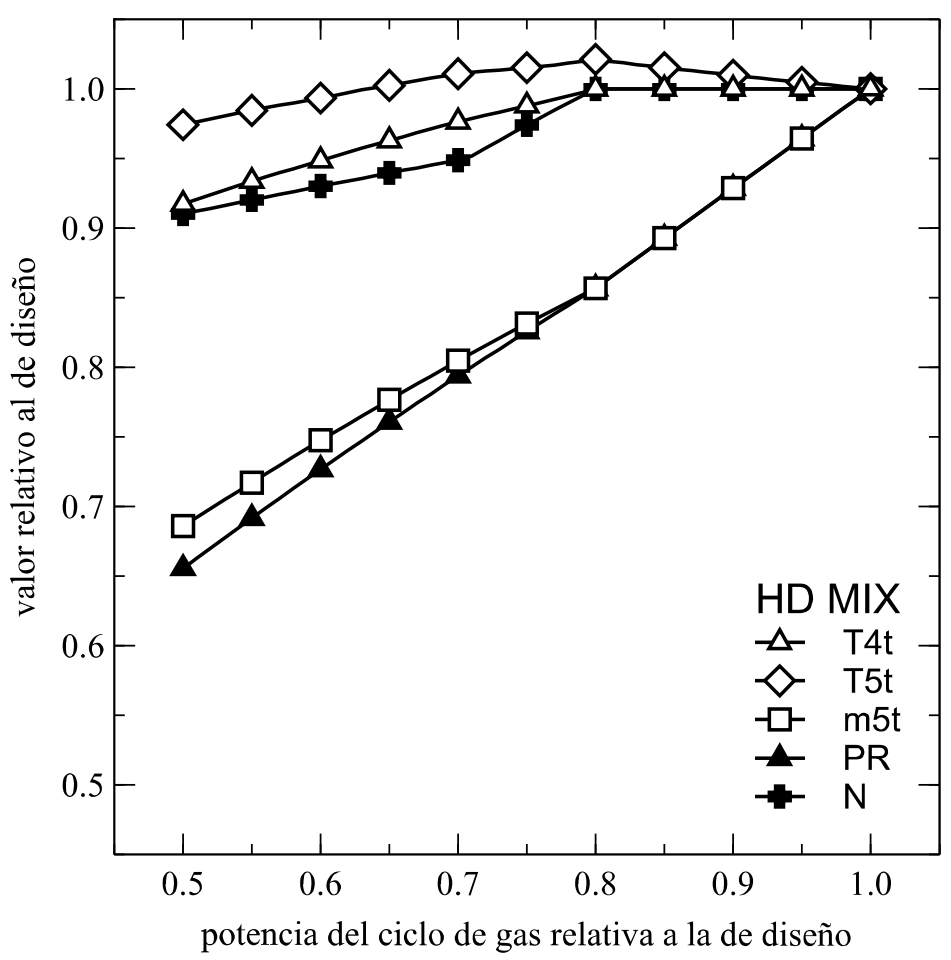

Figura 33. Comportamiento a carga parcial de las variables fundamentales de comportamiento del ciclo semicerrado para turbina monoeje con ley de control mixta, N constante entre 100\% y $80 \%$ de la carga, seguida de control en $T_{4 t}$

Como esta ley alternativa puede no ser factible debido al requisito de operación a la velocidad de sincronismo, se va a definir una ley mixta, denominada HD MIX, en la entre el $80 \%$ y el $100 \%$ de las vueltas se opera a velocidad de giro constante, mientras que a potencias inferiores se controla utilizando $T_{4 t}$. El comportamiento del ciclo semicerrado para esta ley se muestra en la Figura 33. Puede verse que $T_{5 t}$ sube ligeramente al disminuir la potencia, pero vuelve a bajar al entrar en funcionamiento el control de $T_{4 t}$. 


\subsection{Ley de control mediante presión base.}

Existe un método de control adicional que se usa en turbinas de gas de ciclo cerrado. En este tipo de ciclos se puede crear un sistema de almacenamiento de gas que permite regular el gasto másico disponible, variando la presión mínima del ciclo [56], [57]. De esta forma se puede alterar el comportamiento de la turbomaquinaria, permitiendo que esta opere en las mismas condiciones adimensionales pese a la variación en la potencia producida. Junto con el control en $T_{4 t}$, podría permitir la operación a vueltas constantes en todo el rango de funcionamiento.

Dado que el CCSC objeto de este estudio ya tiene un subsistema encargado de separar parte del gasto másico del ciclo, es razonable suponer que este subsistema se podría modificar para regular la presión mínima del ciclo, posiblemente introduciendo un sistema de depósitos intermedio previo al almacenamiento propiamente dicho. El CCSC tiene, no obstante, características únicas que hay que tener en cuenta en este caso:

- La composición del gas recirculado está ligada a las condiciones de presión y temperatura de este, al asumir que se retira el agua mediante condensación. En concreto, la fracción másica de agua se duplica si la presión de condensación se reduce de 1 bar a 0.5 bar, con el consiguiente cambio en las propiedades del gas. Para tratar este efecto adecuadamente habría que calcular la composición y las propiedades del gas para cada presión de operación y realizar las transformaciones correspondientes en los mapas de la turbomaquinaria, de modo que en lo sucesivo se va a asumir que el contenido en agua se puede controlar de forma independiente de la presión de recirculación.

- La relación de presiones de la ASU no coincide con la del compresor del gas recirculado, de modo que, a medida que se reduzca la presión base, se reducirá la presión máxima del ciclo y la relación de presiones de la ASU. 
Esto podría requerir un análisis y un modelo más detallados de este compresor y de su operación a baja carga.

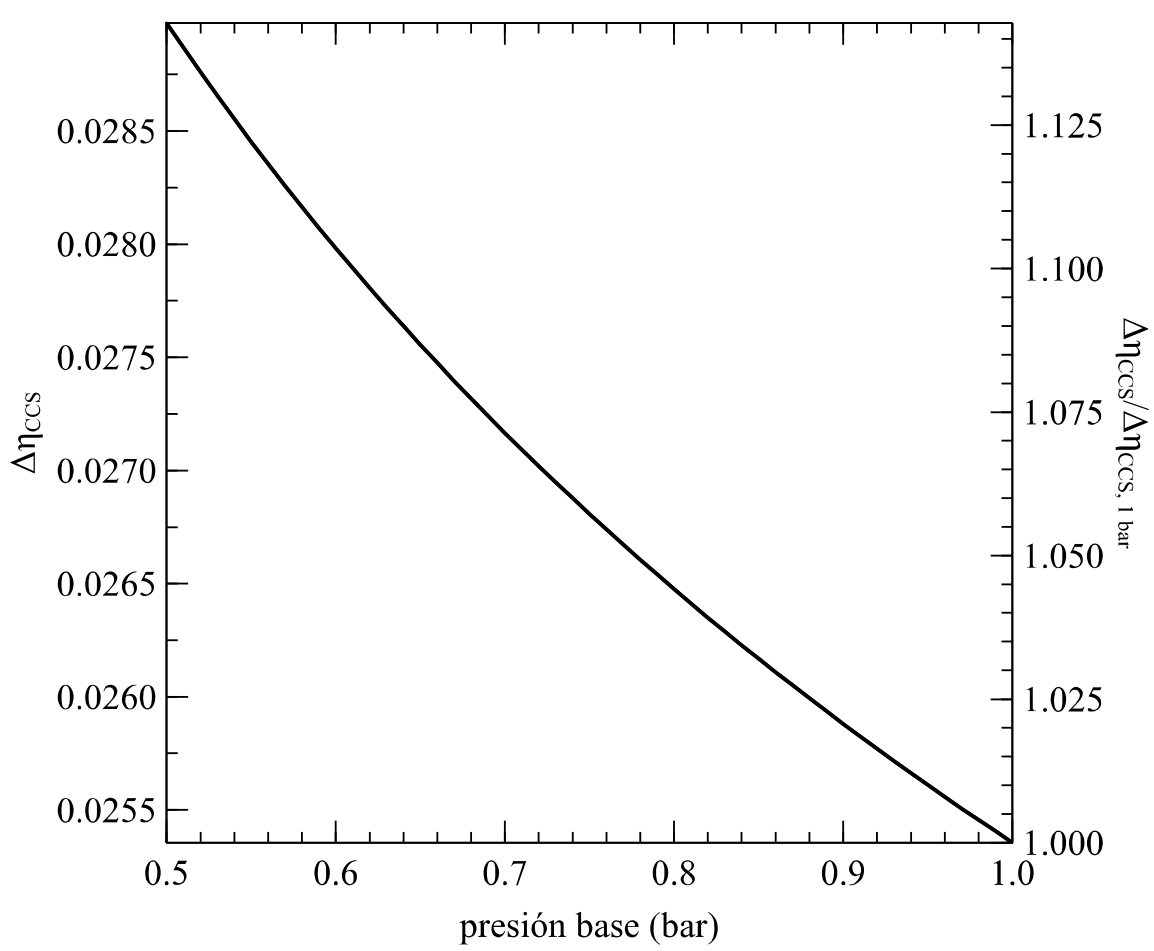

Figura 34. Efecto del compresor adicional para el control de la presión base en la eficiencia del CCS.

- La operación del subsistema de regulación de presión tendrá un cierto coste energético, e impondrá una penalización al ciclo. Este efecto puede simularse de forma aproximada asumiendo la existencia de un compresor adicional en el sistema CCS, que restaura la reducción de presión del gas recirculado para volver a alcanzar los 110 bar de la presión de almacenamiento. La forma en que varía la eficiencia del CCS por incluir este compresor adicional se muestra en la Figura 34, donde puede apreciarse que al reducir la presión del recirculado a 0.5 bar, la penalización en el rendimiento debida al CCS aumenta un 14\% aproximadamente. Como el efecto del CCS en el sistema global es relativamente pequeño, este efecto no se tendrá en cuenta en los análisis posteriores. 


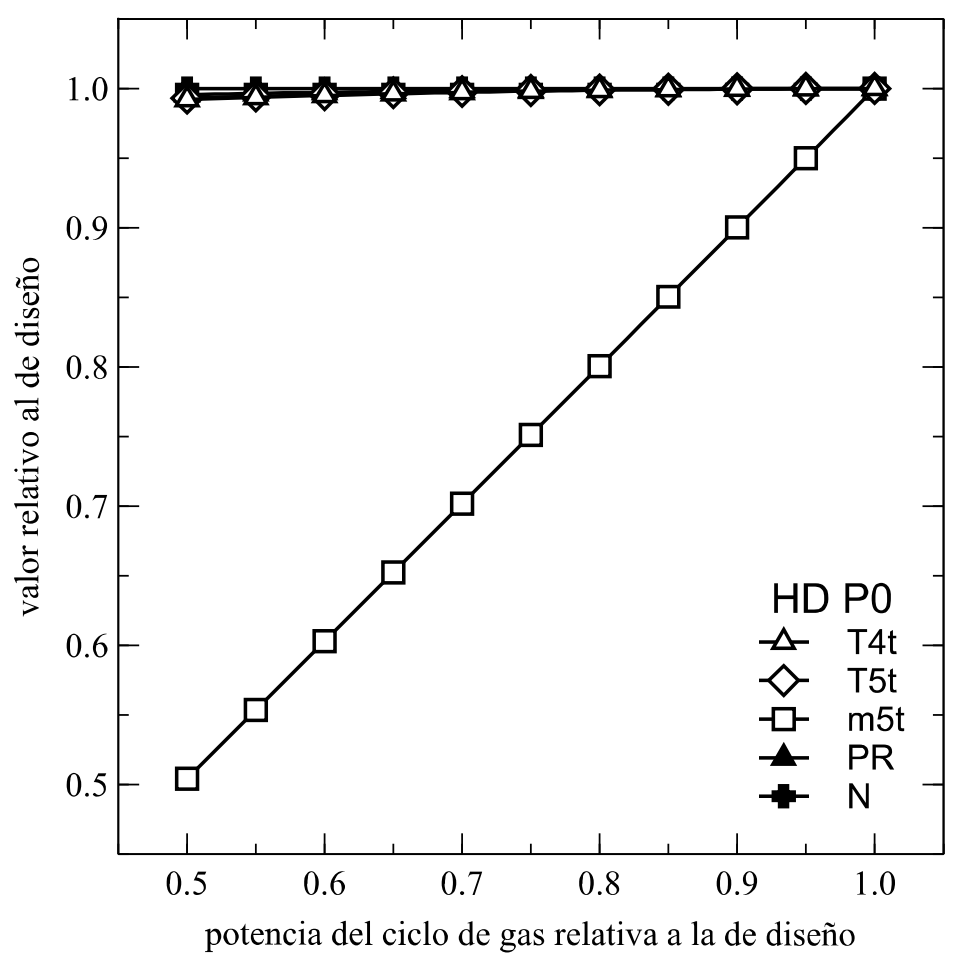

Figura 35. Comportamiento a carga parcial de las variables fundamentales de comportamiento del ciclo semicerrado para turbina monoeje con control en presión base.

De acuerdo con lo ya expuesto, se va a usar una ley de control de una turbina monoeje con regulación de la presión base y control en $T_{4 t}$ para conseguir que la velocidad de giro sea constante para todo el rango de cargas parciales, que se va a denominar HD P0. El comportamiento del ciclo semicerrado se muestra en la Figura 35, donde puede verse que el punto de operación permanece casi constante, salvo por $m_{5 t}$, que sigue la tendencia de la presión del recirculado, por lo tanto, del gasto másico de recirculado, no mostrados en la figura.

\subsection{Comparativa de leyes de control.}

Hasta ahora se ha mostrado el comportamiento de cada ley de control de forma aislada, sin embargo, la selección de una configuración de ejes y una ley de control dependerá de las restricciones impuestas por el generador (la imposición de vueltas constantes en un rango), de la eficiencia obtenida para cada configuración, aunque este factor puede no ser importante si no se espera que el sistema opere de forma regular a carga parcial, y de las condiciones de 
funcionamiento del ciclo de vapor, que requiere que la temperatura de entrada a la turbina, el punto de mínima separación entre los ciclos y la aproximación a la saturación a la salida del economizador se mantengan en valores aceptables.

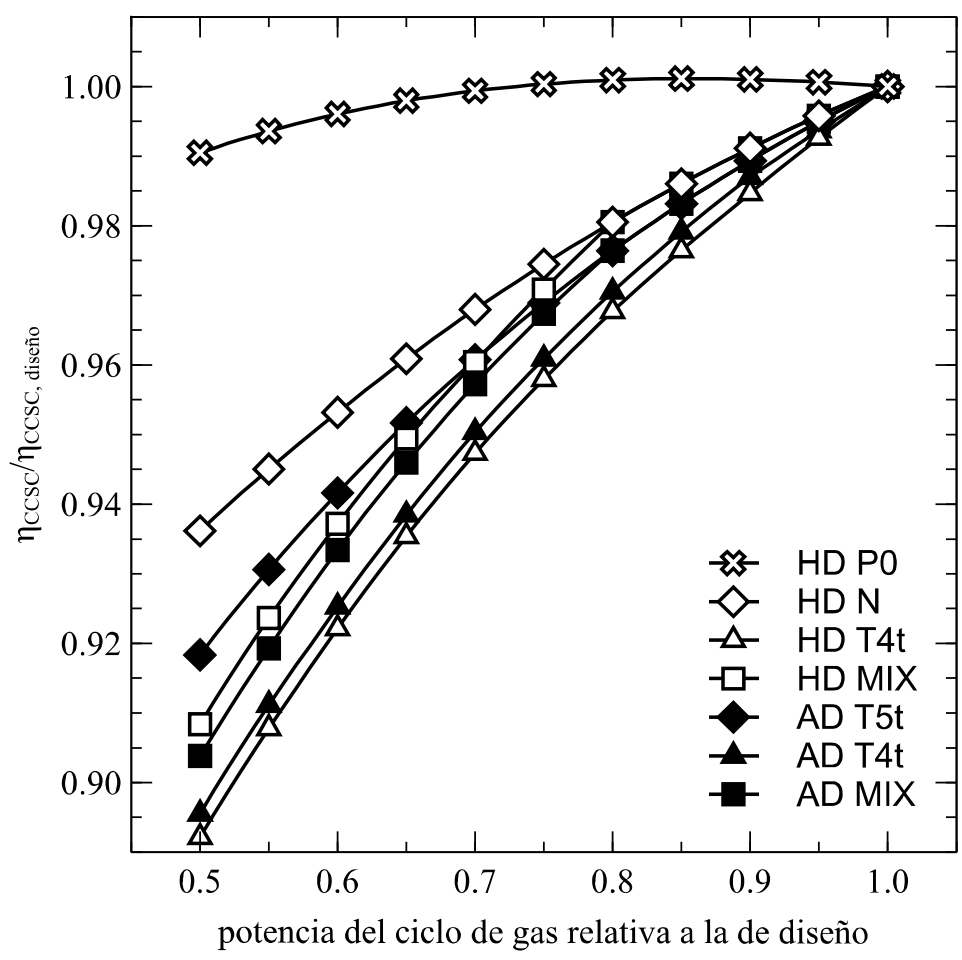

Figura 36. Variación en la eficiencia del CCSC, incluyendo ASU y CCS, con la potencia relativa del ciclo de gas.

En la Figura 36 se muestran los resultados de eficiencia del ciclo completo incluyendo los efectos de la ASU y el CCS, adimensionalizados con la eficiencia del punto de diseño, para cada una de las leyes de control mencionadas anteriormente. La eficiencia de todas las leyes sin control de la presión base baja apreciablemente al reducir la potencia, mientras que la de HD P0 se mantiene muy alta en todo el rango. Es interesante observar que, pese a las pérdidas inducidas por el VIGV/VSV, la eficiencia del caso HD N se mantiene por encima de los casos sin control de presión base, mientras que el HD T4t es el peor de todos, y el HD MIX está entre ambos. Para los casos AD sucede lo contrario, como se pretendía, con el caso AD T5t por encima del AD T4t, con el caso $\mathrm{AD}$ MIX entre los dos. Es interesante señalar que, incluso en el peor de los casos, la eficiencia se mantiene por encima del $89 \%$ del valor original. 

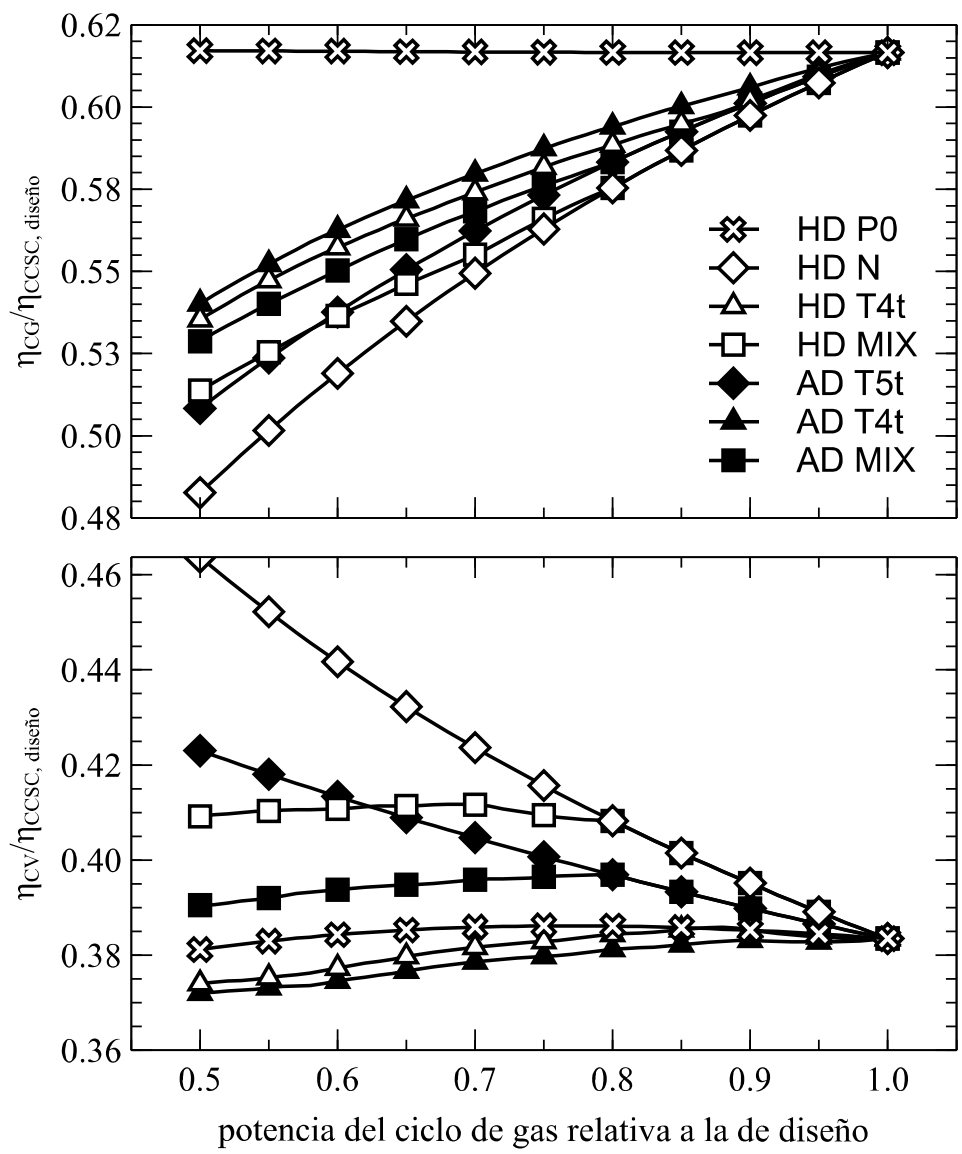

Figura 37. Variación de la eficiencia del ciclo de gas (superior) y vapor (inferior), adimensionalizados con el rendimiento del CCSC, con la potencia relativa del ciclo de gas.

Estos resultados pueden explicarse parcialmente mediante su descomposición en las aportaciones del ciclo de gas y del ciclo de vapor, que se muestran en la Figura 37, las eficiencias de cada ciclo se han adimensionalizado con la eficiencia del punto de diseño del CCSC, sin incluir el efecto de la ASU ni el CCS. En la parte superior de la figura se muestra la eficiencia del ciclo de gas, que sigue el comportamiento esperado. El caso HD T4t se mantiene por encima del HD N, mientras que el AD T4t se mantiene por encima del AD T5t, y la eficiencia del caso HD P0 prácticamente no cambia. La eficiencia del ciclo de vapor, mostrada en la parte inferior de la figura muestra una tendencia muy distinta, la eficiencia del ciclo de vapor de los casos HD N y AD T5t crece considerablemente con la disminución de la potencia de la turbina de gas, más que compensando las pérdidas de eficiencia del ciclo de gas. 


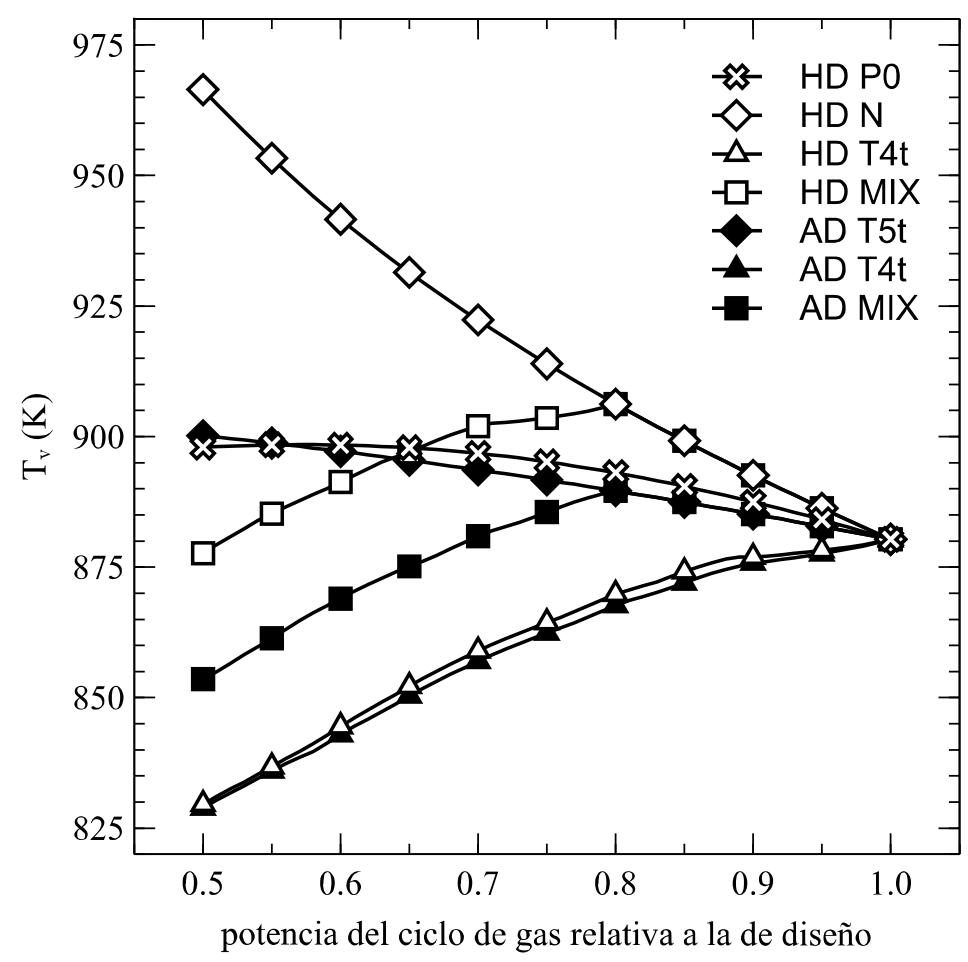

Figura 38. Variación de la temperatura de entrada a la turbina de vapor con la potencia relativa del ciclo de gas.

Los resultados del ciclo de gas están relacionados con la temperatura de entrada a la turbina de vapor, $T_{v}$ que se muestra en la Figura 38. Como puede verse, la $T_{v}$ del caso HD N crece considerablemente a medida que disminuye la potencia, superando con mucho el valor admisible. El caso HD MIX revierte esta tendencia cuando no se ha alcanzado aún un valor muy grande, aunque está por encima de $900 \mathrm{~K}$. En los casos AD T5t y HD P0 también crece la $T_{v}$, aunque más lentamente, quedando muy cerca de $900 \mathrm{~K}$ para carga parcial 0.5. Como se esperaba por la tendencia de $T_{5 t}$ y $m_{5 t}$ de los casos AD T4t y HD T4t, $T_{v}$, decrece al disminuir la potencia, y lo mismo sucede en los casos mixtos relacionados.

Aunque el ciclo de vapor no se ha modelado con mucho detalle, es de interés comprobar el comportamiento de otras variables de relevancia para su comportamiento a carga parcial. La aproximación a la saturación a la salida del economizador es un parámetro a seguir, indicando si se está produciendo la evaporación dentro del economizador y produciendo una posible transición del punto de mínimo acercamiento entre los ciclos [51]. La variación de este 
parámetro se muestra en la Figura 39, donde puede verse que no se produce evaporación para ninguno de los casos estudiados, aunque los casos AD T4t y HD T4t están bastante cerca.

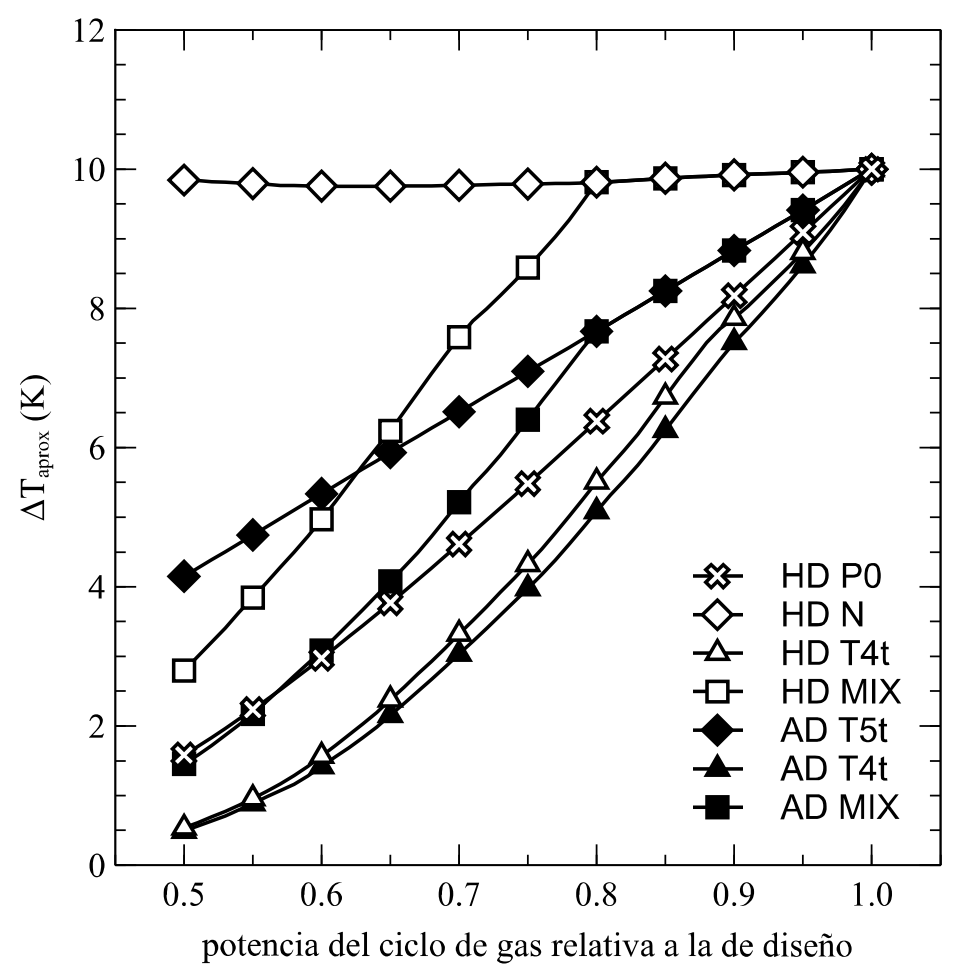

Figura 39. Variación de la aproximación a la saturación a la salida del economizador con la potencia relativa del ciclo de gas.

Otra variable de interés es el mínimo acercamiento entre los ciclos, pinch point, cuya evolución se muestra en la Figura 40. Puede verse que en todos los casos este acercamiento se reduce considerablemente, especialmente en el caso HD PO. Aunque esto se suele relacionar con una menor eficiencia del intercambio de calor [51], en estos casos la eficiencia global obtenida es muy alta. 


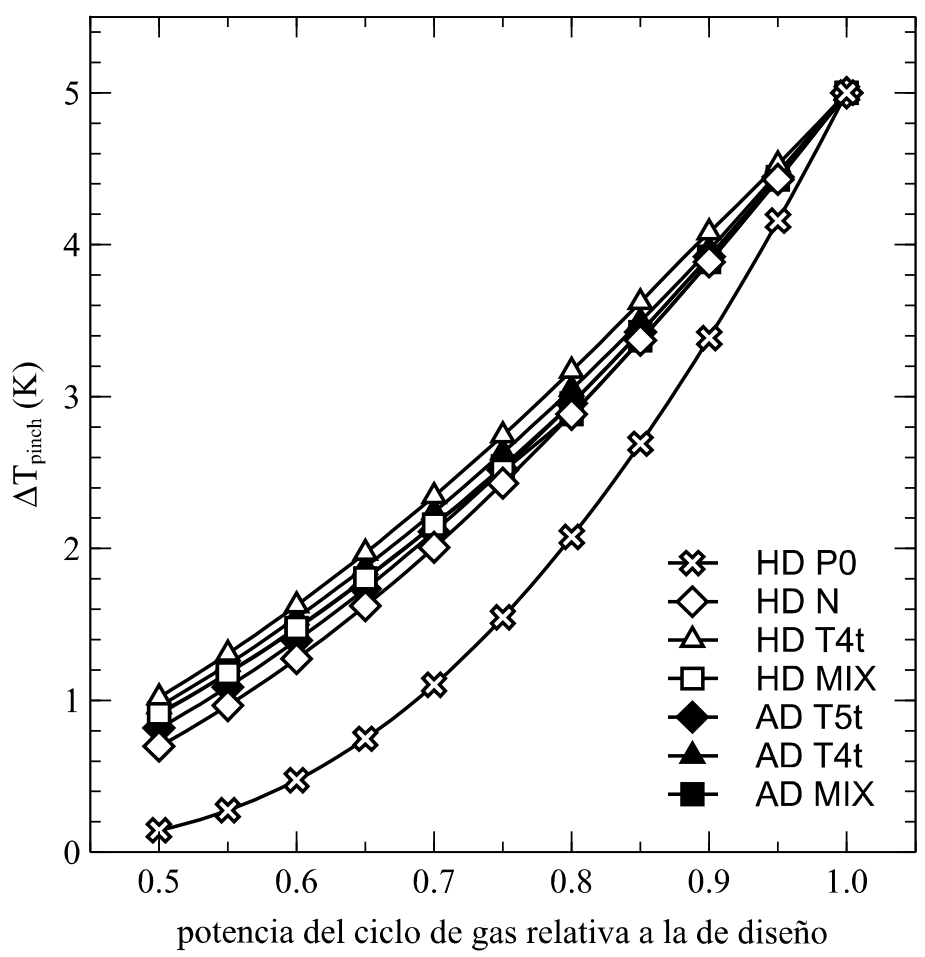

Figura 40. Variación del acercamiento mínimo entre los ciclos con la potencia relativa del ciclo de gas. 


\section{ESTUDIO DE LA CÁMARA DE COMBUSTIÓN.}

En esta sección, como caso particular de adaptación de componentes, se pretende evaluar el proceso de oxicombustión diluida y sus consecuencias en el diseño preliminar y el comportamiento de la cámara de combustión, en comparación con cámaras de combustión con aire. Para ello se va a construir un modelo simplificado de la cámara usando redes de reactores químicos, consistente en un reactor de flujo continuo, bien agitado, al que después se va a acoplar un reactor tipo Plug Flow. Esta aproximación implica un leve aumento del nivel de detalle respecto al de los modelos mostrados con anterioridad, pero conserva parte de sus ventajas: el número de parámetros necesarios para definirla son pocos y no requiere mucha potencia de cálculo. Esto permite realizar análisis paramétricos normalmente imposibles con modelos más detallados.

Al tratarse de un proceso de oxicombustión diluida, la construcción de este modelo simplificado presenta problemas adicionales, porque será necesario seleccionar cuáles son los parámetros de diseño apropiados, al no poder utilizar los de cámaras de combustión convencionales. Para ello será necesario analizar de forma general el comportamiento de este modelo, y la posible influencia de los parámetros del ciclo semicerrado.

Una vez seleccionados los parámetros de diseño del modelo se construirán y compararán modelos para la cámara de combustión del CCSC y para cámaras de combustión con aire. Esta comparación debería mostrar las diferencias entre ambos procesos y si se pueden adaptar cámaras de combustión con aire para su funcionamiento en oxicombustión o si es necesario diseñarlas desde cero. Como en el caso del análisis de actuaciones, este tipo de análisis simplificado es un paso necesario antes de llevar a cabo cualquier estudio más detallado. 


\subsection{Modelo de simulación.}

El comportamiento de la cámara de combustión se va a simular mediante una red de reactores químicos acoplados. Esta aproximación permite el uso de esquemas de cinética química relativamente complejos con un bajo coste computacional, siendo muy apropiado para el diseño preliminar de cámaras de combustión, mientras que los modelos CFD requieren esquemas simplificados y el diseño detallado de la cámara; un modelo estándar para la combustión de gas natural es el GriMech 3.0 [58], con 325 ecuaciones. Los componentes del modelo se presentan a continuación, el lector puede encontrar más detalles en la literatura especializada, por ejemplo, [59].

Un reactor de flujo continuo, bien agitado, WSR (Well Stirred Reactor) es un reactor cero-dimensional de volumen constante, $V_{W S R}$, en el que la composición, la temperatura y la presión son uniformes. En este estudio se van a asumir: macro y micro-mezclado perfectos, presión constante, proceso adiabático y estado estacionario.

Puede obtenerse un tiempo de residencia medio de paso por un reactor suponiendo que este tiene un área de paso constante $A$, una longitud $L$ (de modo que el volumen del reactor es $V=L A$ ) y que el fluido se mueve a través de él con una velocidad media $U$, en la forma

$$
t_{\text {res }}=\frac{L}{U}=\frac{L}{U} \frac{A}{A} \frac{\rho}{\rho}=\frac{\rho V}{m_{\text {out }}}=\frac{m_{\text {WSR }}}{m_{\text {out }}} .
$$

Donde $\rho$ es la densidad dentro del reactor, $m_{\text {WSR }}$ es la masa que hay dentro del reactor y $m_{\text {out }}$ el gasto másico que sale de él. También puede escribirse directamente en función de las condiciones de presión, $P$, y temperatura, $T$, en su interior:

$$
t_{\text {res,WSR }}=\frac{m_{\text {WSR }}}{m_{\text {out }}}=\frac{V_{\text {WSR }}}{m_{\text {out }}} \frac{P}{R T} .
$$


Las reacciones de combustión también tienen un tiempo químico característico, dependiente de las concentraciones de los reactantes y de la temperatura, siendo la dependencia con esta última típicamente de forma exponencial. Para que la combustión sea posible, el tiempo de residencia tiene que ser al menos del mismo orden que el tiempo químico; para tiempos de residencia muy grandes se alcanzarán las condiciones de equilibrio químico. Si se disminuye el tiempo de residencia (reduciendo el volumen o aumentando el gasto másico), el progreso de la reacción será cada vez menor, hasta un cierto tiempo en el que la combustión se vuelva inestable, produciéndose el apagado. Por ejemplo, en cámaras de combustión de turbinas de gas este tiempo es del orden de $0.5 \mathrm{~ms}$ [60]. Aunque puede usarse un solo WSR para modelar la cámara de combustión completa [61], [62], es más frecuente usarlo para fijar sus características de estabilidad [60].

Un reactor tipo Plug Flow, PFR (Plug Flow Reactor), es un reactor unidimensional con propiedades constantes en la dirección perpendicular al flujo. Si se desprecian los cambios en la cantidad de movimiento axial y energía cinética, se pueden aplicar las ecuaciones de conservación de la energía y de las especies a una rebanada infinitesimal. Junto con las suposiciones de presión constante, proceso adiabático y estado estacionario, el PFR puede representarse mediante una serie de WSR de tamaño infinitesimal [63]; esto permite la inyección de gas adicional de dilución en cualquier estación. Para definir el tamaño del PFR puede darse su volumen total, que será la suma de los de los WSR que lo componen. El tamaño del PFR también podría fijarse mediante el tiempo total de residencia en su interior, que es la suma de los tiempos de residencia de los WSR que lo componen.

En la literatura científica pueden encontrarse distintas redes de reactores químicos y distintos métodos de ajustar sus parámetros para estudiar las cámaras de combustión de las turbinas de gas. Por ejemplo, el tiempo de residencia en cada reactor se puede fijar usando información empírica como las emisiones de 
$\mathrm{CO}, \mathrm{NO}_{\mathrm{x}}, \mathrm{o}$ hidrocarburos inquemados, UHC, [64], usando visualización experimental del flujo [63] o mediante la comparación con resultados de mecánica de fluidos computacional [65].

En este estudio se han considerado dos posibles configuraciones, teniendo en cuenta la forma en que se puede distribuir el aire o el gas recirculado dentro de la cámara:

- Tipo 1. Los métodos estándar de diseño de cámaras de combustión para turbinas de gas de aerorreactores [66] dividen la cámara en una zona primaria (ZP), donde la llama está anclada mediante recirculación, una zona intermedia (ZI), donde los gases de la ZP continúan su combustión mientras se mezclan con más aire, y una zona de dilución (ZD) donde el aire restante se mezcla con el gas de la ZI. Este aire se mezcla de forma que se mantenga una temperatura uniforme, igual a la de salida de la ZP. La temperatura de la carcasa de la cámara se controla mediante refrigeración pelicular, requiriendo la inyección de aire adicional.

- Tipo 2. En las turbinas con mezcla pobre y premezclado, aproximadamente el $80 \%$ del aire disponible se inyecta en la ZP, reduciendo o eliminando las necesidades de refrigeración pelicular o los agujeros de la ZI. La refrigeración de la carcasa de la cámara se consigue mediante convección interna, refrigeración mediante impacto $\mathrm{y}$ revestimientos de barrera térmica; las turbinas de gas de ciclos combinados pueden incluir refrigeración en ciclo cerrado usando vapor para mejorar la eficiencia.

Se va a utilizar un modelo genérico capaz de simular ambas configuraciones, asumiendo premezcla perfecta. La combustión se va a simular usando una red muy simple consistente en un WSR seguido de un PFR. Esta red está basada en el estudio clásico de Bragg de 1953 [67], y se sigue aplicando a la combustión en turbinas de gas [68], [69]. También se ha usado para simular oxicombustión en unas condiciones ligeramente diferentes a las de este estudio [70], pero no se ha 
encontrado un análisis sistemático de su comportamiento en la literatura. El uso de una red con más reactores químicos añadiría más parámetros al modelo, sin aumentar su precisión necesariamente [71].

Los gastos másicos disponibles para la combustión se van a fijar usando los mismos parámetros que en el estudio del ciclo:

- Para la oxicombustión se usarán $m_{\mathrm{ASU}}, \varphi, f_{\text {est }} \mathrm{y} r$. Una fracción $K_{Z P}$ del gas recirculado se tomará como perfectamente premezclada con el combustible y el oxidante antes de la entrada de esta mezcla en la ZP.

- Para la combustión con aire, se usarán $m_{\text {aire, }}$ y una variable similar a $f_{\text {est }}$, la relación aire-combustible estequiométrica, far ${ }_{\text {est }}$. Para conseguir un modelo similar al de oxicombustión, el gasto de aire total se dividirá en la parte de oxidante necesario para la combustión estequiométrica y la parte de aire en exceso de dicha combustión. Una fracción $K_{Z P}$ del aire en exceso se premezclará con la parte estequiométrica de oxidante y de combustible antes de la inyección en la ZP.

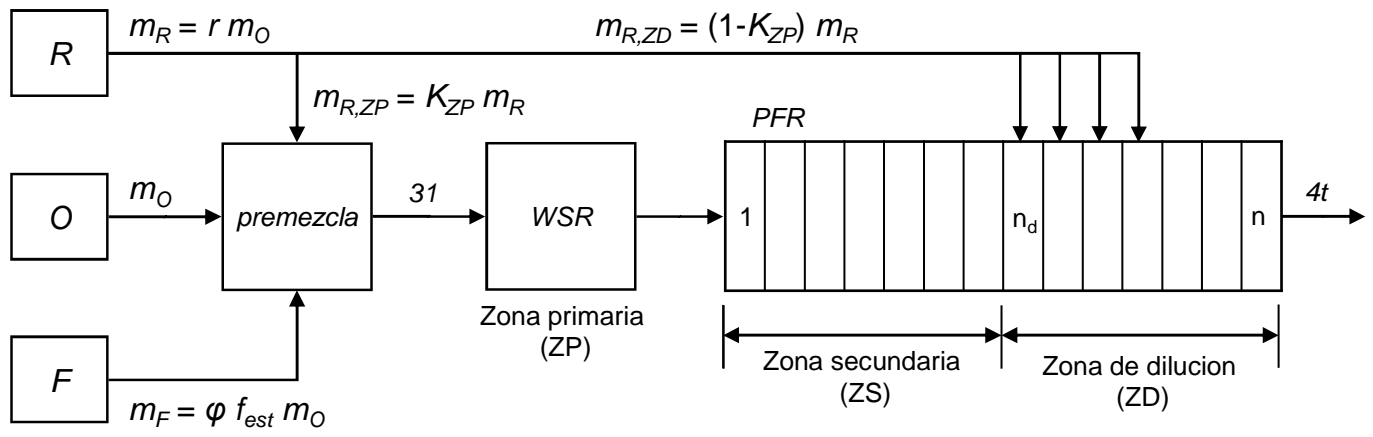

Figura 41. Esquema de una cámara de combustión diluida. $O, R$ representan al aire de combustión estequiométrica y al exceso de aire, respectivamente, o al gasto másico de la ASU y al gas recirculado que llega a la cámara. F representa al combustible, un gas natural.

La ZP se modelará mediante un WSR, con un volumen asociado $V_{Z P}$, que fijará las características de combustión de la cámara. Los productos de combustión de la ZP continuarán el proceso en un PFR con un volumen asociado $V_{P F R}$. La inyección del gas (aire o recirculado) restante comenzará en una cierta estación del PFR, $n_{d}$, y puede seguir cualquier distribución másica deseada. A la parte del 
PFR entre la ZP y el inicio de la inyección de gas restante se la denominará zona secundaria, ZS, y al resto zona de dilución, ZD. Un esquema del modelo se muestra en la Figura 25.

Se va a definir un área de paso de referencia, $A_{\text {ref, }}$, mediante el número de Mach

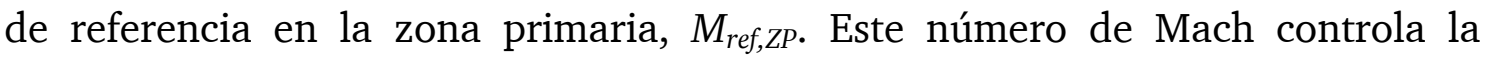
aerodinámica de la combustión y la pérdida de presión en la cámara; un Mach de referencia igual a 0.05 es representativo de este tipo de cámaras [72]. El gasto másico que pasa por la zona primaria puede escribirse en la forma

$$
m_{Z P}=\rho_{31} U_{r e f} A_{r e f},
$$

donde $U_{\text {ref }}$ es la velocidad de referencia y $\rho_{31}$ es la densidad en la sección de entrada a la zona primaria. El área de referencia puede obtenerse a partir de las condiciones de entrada y el número de Mach de referencia, aproximando las condiciones estáticas mediante las de remanso, como

$$
A_{\text {ref }}=\frac{m_{Z P}}{M_{r e f}} \frac{\sqrt{R_{31} T_{31}}}{P_{31} \sqrt{\gamma_{31}}} .
$$

Como el volumen de cada reactor es conocido, puede obtenerse una estimación de la longitud del WSR a partir del área de referencia

$$
L_{W S R}=\frac{V_{W S R}}{A_{\text {ref }}} .
$$

Se puede hace lo mismo para el PFR

$$
L_{P F R}=\frac{V_{P F R}}{A_{\text {ref }}},
$$

obteniendo una estimación del tamaño de la cámara.

Al final de la cámara, la temperatura debería ser la definida por el punto de diseño del ciclo e, idealmente, los productos de combustión deberían tener la composición de equilibrio. En las configuraciones tipo 1, las reacciones se 
consideran congeladas después de la inyección del aire de dilución. Este efecto no se debe a la baja velocidad de la cinética química asociada con la reducción de temperatura debida a la dilución, dado que el consumo de CO es rápido en combustión homogénea por encima de 1300K [73], o cerca de la capa límite de la pared [74]. La detención del consumo de CO se ha relacionado con la presencia de aire de refrigeración pelicular o con no uniformidades en la distribución de aire y combustible [72], [74]. En las configuraciones tipo 2 se suele asumir que las reacciones proceden hacia el equilibrio a lo largo de la cámara.

Como este estudio solo tiene en cuenta los efectos de la cinética química, no puede predecir la concentración de $\mathrm{CO}$ a la salida, que debería de estar entre la de equilibrio a la temperatura a la salida de la ZP, para una configuración tipo 1, y la de equilibrio a la salida de la cámara, para una configuración tipo 2. La comparación entre oxicombustión y combustión con aire debería servir para disminuir la incertidumbre, asumiendo que los efectos no derivados de la cinética química son similares.

Para realizar el estudio, se va a utilizar al paquete de cinética química Cantera [75], desarrollado por D G. Goodwin, del California Institute of Technology. Cantera es un paquete de software de código abierto escrito en $\mathrm{C}++$ con técnicas de orientación a objetos, con interfaces escritas para Python y Matlab, que permiten el modelado rápido de problemas de cinética química. El programa también permite enlazar las entradas y salidas de distintos reactores para formar redes complejas. Se puede emplear cualquier esquema estándar de cinética química, o crear modelos simplificados. Se va a usar el modelo GriMech 3.0 [58], usado habitualmente para la combustión de gas natural.

\subsection{Modelo auxiliar.}

Como ya se ha visto al analizar el balance másico del ciclo, el gas recirculado puede contener una cierta cantidad de oxidante, dada por 


$$
Y_{A S U, R}=\frac{(1-\varphi)\left(1-w_{r}\right)}{1-\varphi+\varphi k_{g s}}
$$

de modo que, si la combustión se realiza con exceso de oxidante $(\varphi<1)$, el gas recirculado inyectará de nuevo la fracción másica correspondiente, amplificada por la relación entre los gastos másicos de gas recirculado y de la ASU, $r$, con lo que la relación de equivalencia real en la cámara, $\Phi$, que es la que controla el proceso de combustión, no coincidirá con el parámetro utilizado en el ciclo $\varphi$.

La masa total de oxidante presente será

$$
m_{O}=\left(1+r Y_{A S U, R}\right) m_{A S U} .
$$

Y la relación de equivalencia real

$$
\Phi=\frac{1}{f_{\text {est }}} \frac{m_{F}}{m_{O}}=\frac{\varphi}{1+r Y_{A S U, R}}
$$

Si se elimina la parte correspondiente al oxidante del gas recirculado, se obtiene un gas de dilución, $D$, cuyo gasto másico se puede relacionar con el gasto másico de oxidante mediante una relación de dilución, $d$, en la forma

$$
d=\frac{m_{D}}{m_{O}}=\frac{1+r}{1+r Y_{A S U, R}}-1
$$

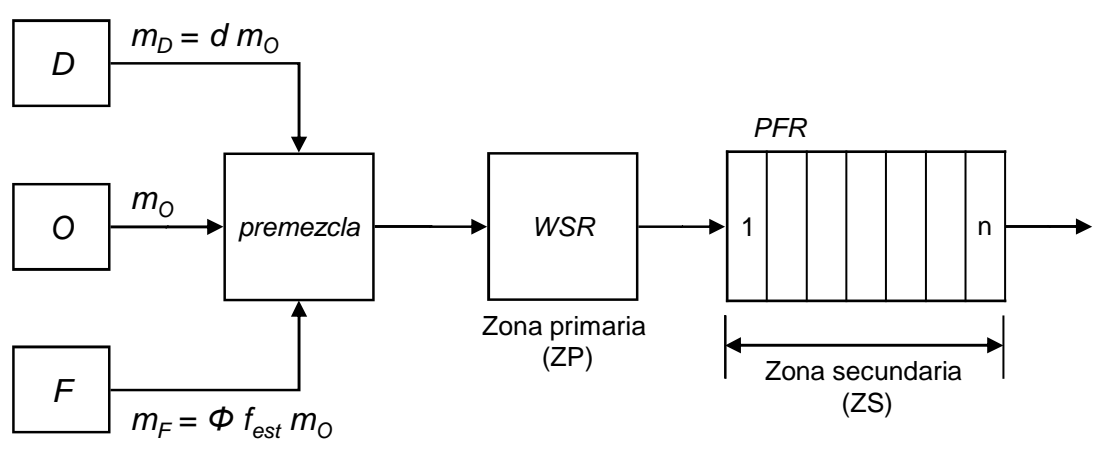

Figura 42. Esquema de una cámara de combustión utilizando la representación $\Phi, d$.

El modelo del proceso de combustión usando la representación $\Phi, d$, sin dilución adicional, cuyo esquema se muestra en la Figura 42, se utilizará como modelo 
auxiliar para analizar ciertos aspectos del proceso. Esta representación puede simplificar la comparación con la combustión con aire, que se puede interpretar como una combustión diluida, utilizando oxígeno como oxidante y nitrógeno como diluyente, con una relación de dilución constante, $d_{\text {aire }}$. La composición simplificada de $79 \% \mathrm{~N}_{2}$ y $21 \% \mathrm{O}_{2}$ resulta en $d_{\text {aire }}=3.29$. Si se usa $d_{\text {aire }}$ para la combustión con $\mathrm{CO}_{2}$ como diluyente, la fracción volumétrica de $\mathrm{O}_{2}$ resultante, $X_{O 2}=30 \%$, resulta ser el valor mínimo requerido para la combustión estable por Ditaranto y Hals [28]; también está muy cerca de los valores usados por Tan et al (28\%) [26] y por Andersson y Johnsson (27\%) [27].

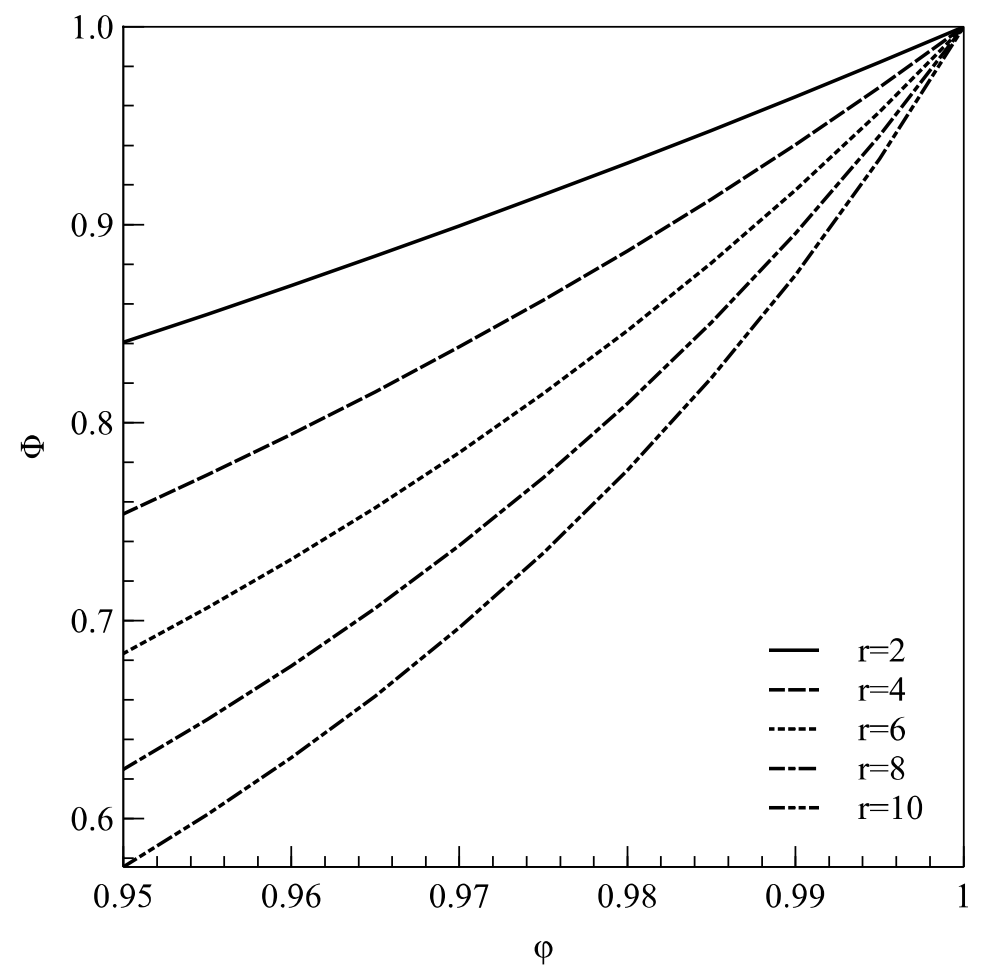

Figura 43. Relación de equivalencia real, $\Phi$, frente a relación de equivalencia del ciclo, $\varphi$, y relación de recirculación, $r$, para $w_{\text {est }}=0.41476, f_{\text {est }}=0.253815, w_{r}=0.028$.

Como ya se ha visto en el modelo de la cámara, la combustión con aire se puede expresar en una forma similar a la representación $\varphi, r$, mediante la división del gasto másico total de aire en su parte de oxidante, $m_{\text {aire, } o}$ y la parte de exceso de aire, $m_{\text {aire,ex. }}$ Al usar el gasto de oxidante para adimensionalizar los demás, el gasto másico de combustible puede darse mediante una relación de equivalencia del ciclo, $\varphi$, que puede tomarse igual a uno para combustión pobre, y una 
relación entre el gasto másico de aire en exceso y el gasto másico de aire oxidante, dada por la variable $r$.

La relación entre la relación de equivalencia real y de ciclo para los valores de las constantes obtenidas en el apartado de balance másico del ciclo se muestra en la Figura 43, donde está claro que los valores altos de $r$ amplifican considerablemente el contenido en oxidante y el exceso de oxígeno, dando valores cercanos a los reportados por otros autores [26], [27], [28] como necesarios para tener buenas características de combustión.

\subsection{Comportamiento de la zona primaria.}

Como ya se ha mencionado, se puede asociar un tiempo químico con la combustión dentro del WSR; cuando el tiempo de residencia en el reactor es menor que este tiempo químico la combustión estable no es posible y se produce el apagado (blow-out). El mínimo tiempo de residencia para el que es posible la combustión estable se denominará tiempo de residencia de apagado incipiente, y se representará mediante $t_{\text {res, } L B O}$, para enfatizar que solo la combustión pobre, (lean), es de interés práctico en este caso.

Para la combustión con aire, esta condición se suele representar como una sola curva en una gráfica de relación de equivalencia frente a la inversa del tiempo de residencia. La región de combustión estable está localizada a la izquierda de esta curva. Como la oxicombustión tiene la cantidad de diluyente como parámetro adicional, se tendrá una familia de curvas características de estabilidad.

La Figura 44 muestra las curvas características de estabilidad de la ZP para una presión fija de 30 bar y una temperatura de entrada de $700 \mathrm{~K}$. Para facilitar la comparación, se ha usado oxígeno puro como oxidante, metano como combustible y nitrógeno o dióxido de carbono puros como diluyentes, la composición se ha fijado usando la representación $\Phi$, $d$. La línea discontinua corresponde a la combustión con aire, con $\mathrm{N}_{2}$ como diluyente y $d=d_{\text {air }}=3.29$; 
el tiempo de residencia típico de turbinas de gas estándar está entre 0.1 y $1 \mathrm{~ms}$ [60]. La zona estable es la correspondiente a tiempos de residencia altos, situada a la izquierda de cada curva. Las líneas sólidas corresponden al diluyente $\mathrm{CO}_{2}$, precisando mayor tiempo de residencia para los mismos valores de $\Phi, d$, que el caso con $\mathrm{N}_{2}$. También puede verse que el incremento de $d$ aumenta considerablemente el tiempo de residencia de apagado incipiente para un mismo diluyente y el mismo valor de $\Phi$. Sin embargo, la comparación de los tiempos de residencia en estas condiciones no es adecuada para el CCSC. Mientras que en una cámara de combustión con aire $\Phi_{Z P}$ se encuentra entre 0.5 y 0.6 [66], [72], en el ciclo objeto de este estudio varía con $r$.

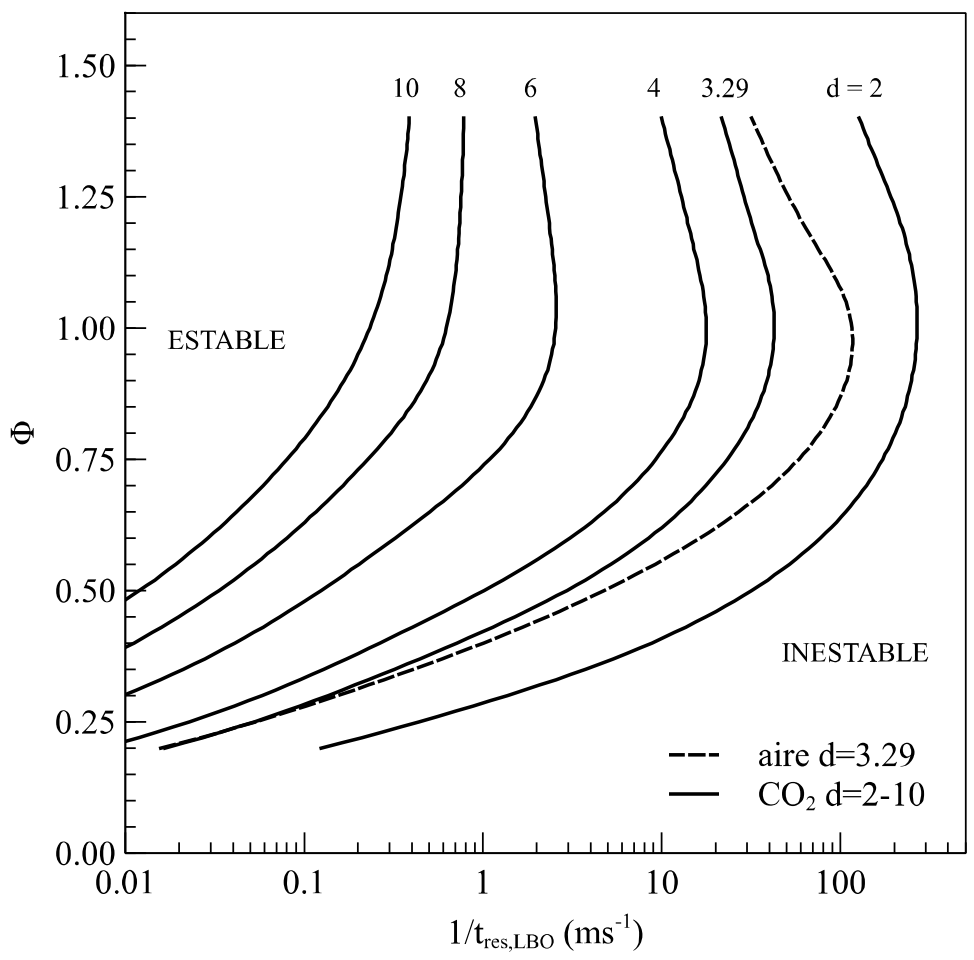

Figura 44. Curvas características de estabilidad de la ZP como función de $\Phi$ y d, para combustión con aire y combustión diluida con $\mathrm{CO}_{2}$.

Se pueden obtener curvas características de estabilidad en una forma alternativa si se sustituye $\Phi$ por la temperatura de equilibrio correspondiente a la composición fijada por cada valor de $\Phi$ y $d, T_{e q}$. Esta temperatura es representativa de la temperatura real en la ZP, controlando el proceso de combustión a través de los factores de cada reacción química. Estas curvas 
características alternativas se muestran en la Figura 45, donde el efecto de $d$ se ve muy reducido. La combustión estable con $\mathrm{CO}_{2}$ como diluyente, con $t_{\text {res }}$ en el rango 0.1-1 ms, requiere $d \leq 6$ y una $T_{e q}$ ligeramente inferior a la de combustión con aire.

A la vista de estos resultados parece adecuado el uso de la temperatura de equilibrio en la zona primaria, $T_{e q, Z P}$, como una variable de diseño, fijando la fracción de aire/gas recirculado que hay que mezclar con el diluyente y el oxidante, dada por $K_{Z P}$. De este modo, la estabilidad de la cámara vendrá fijada por su volumen y por la temperatura de apagado incipiente $T_{\text {eq,LBO }}$. La $T_{\text {eq,ZP }}$ de diseño tendrá que ser mayor que $T_{e q, L B O}$, de modo que la diferencia entre ambas será el margen de estabilidad $\triangle T_{L B O}$. Este margen de estabilidad se podrá usar en lugar del volumen de la ZP para fijar el comportamiento de la ZP.

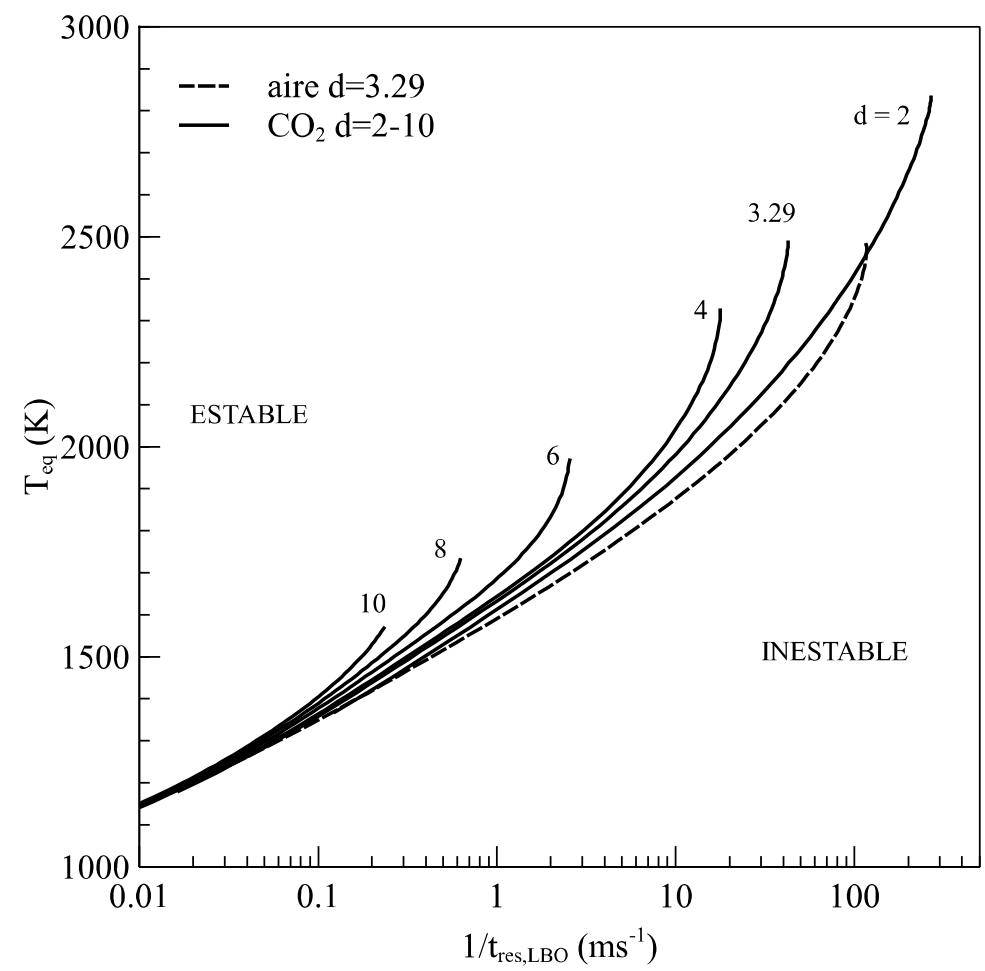

Figura 45. Curvas características de estabilidad de la ZP y como función de $T_{\text {eq }}$ y d, para combustión con aire y combustión diluida con $\mathrm{CO}_{2}$. 


\subsection{Comportamiento de la zona secundaria.}

La cámara de combustión con aire, combustible gaseosos y premezcla, sin dilución adicional, descrita por Snyder et al [76] se va a usar como base para comparar la evolución de combustión diluida con aire y $\mathrm{CO}_{2}$ en la zona secundaria.

Las condiciones de carga base, o BL (base load), dadas son: presión a la entrada 19 bar, temperatura a la entrada $720 \mathrm{~K}$, relación de equivalencia de la ZP, $\Phi_{B L}=$ $0.51\left(T_{e q, B L}=1820 \mathrm{~K}\right)$, tiempo total de residencia $6 \mathrm{~ms}$. También se tiene la relación de equivalencia de apagado incipiente $\Phi_{L B O}=0.45\left(T_{\text {eq, LBO }}=1700 \mathrm{~K}\right)$. Como no hay inyección de diluyente adicional, esta cámara de combustión puede representarse usando el modelo auxiliar, con oxígeno como oxidante, una composición en la ZP dada por $\Phi$ y $d=d_{\text {aire }}=3.29$ y nitrógeno como diluyente. Dado que en [76] no se especifica el combustible utilizado, se asumirá que se trata de metano, lo que es compatible con los resultados de la referencia. El tamaño de la ZP se fijará usando $\Phi_{L B O}$ y el gasto másico para la carga base, 2.6 $\mathrm{kg} / \mathrm{s}$.

En primer lugar, se van a comparar las emisiones de monóxido de carbono obtenidas mediante el modelo con las obtenidas de [76], representadas en la Figura 46. Las emisiones obtenidas mediante el modelo son aproximadamente las correspondientes al equilibrio químico a la salida, presentando la misma tendencia que las experimentales para temperaturas superiores a $1800 \mathrm{~K}$. La combustión incompleta mostrada por los resultados experimentales por debajo de $1800 \mathrm{~K}$ es típica de las turbinas de gas, y se puede relacionar con diversas causas no incluidas en el modelo WSR/PFR [72], [74].

En turbinas de bajas emisiones, la existencia de $\mathrm{CO}$ fuera del equilibrio se suele atribuir a defectos en el premezclado de aire y combustible [74], [76], que dan lugar a zonas localmente ricas en combustible, que pueden interaccionar con la turbulencia y/o las paredes, congelando las reacciones de consumo de CO. 
Algunos autores han propuesto medir la calidad del premezclado mediante la cantidad de CO por encima del equilibrio [73]. Otra posible causa es la mezcla del flujo primario con el flujo de refrigeración pelicular o el de dilución. La tendencia en turbinas de bajas emisiones lleva a reemplazar la refrigeración pelicular de la carcasa mediante refrigeración convectiva y por impacto [72]. Esto es posible gracias al uso de temperaturas moderadas en la zona primaria (inferiores a $1850 \mathrm{~K}$ ), una condición necesaria también para obtener bajas emisiones de $\mathrm{NO}_{\mathrm{x}}$.

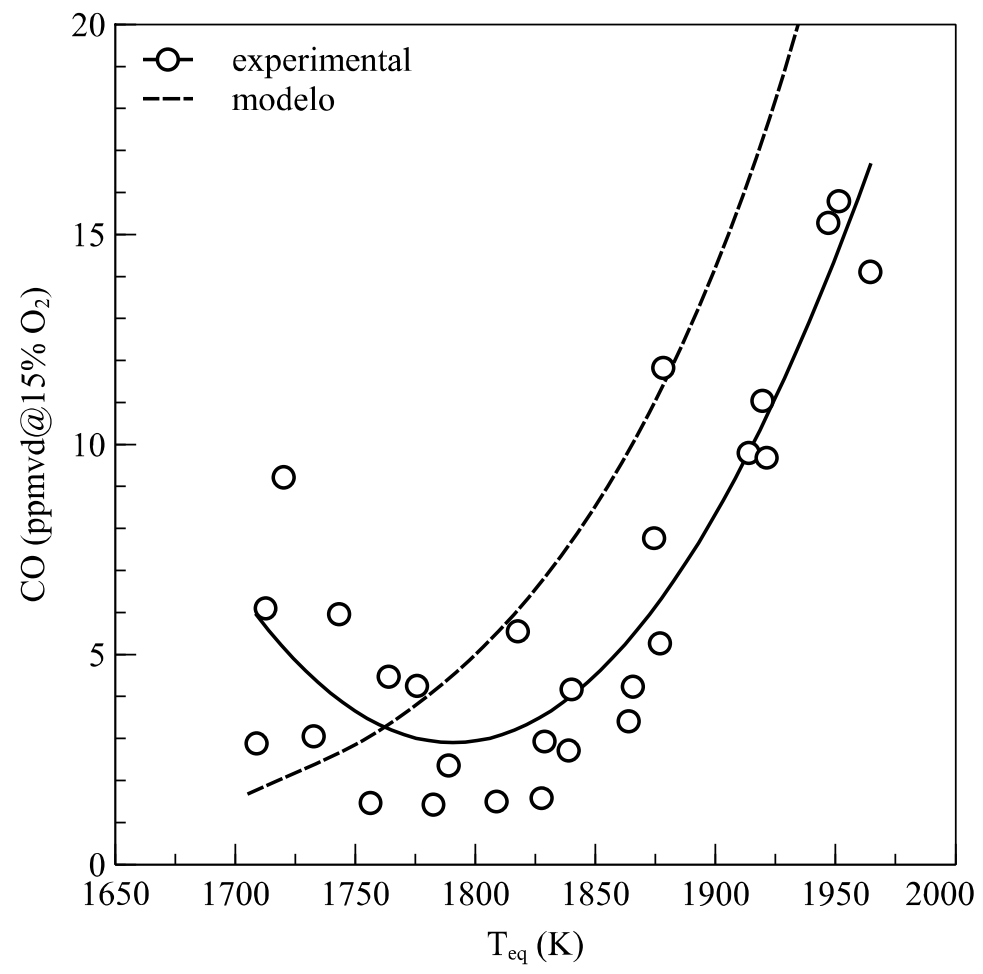

Figura 46. Comparación entre las emisiones de CO experimentales [76], y las obtenidas mediante el modelo.

En la Figura 47 se han representado las emisiones de óxidos de nitrógeno $\mathrm{NO}_{\mathrm{x}}$ del modelo para un tiempo de residencia de $6 \mathrm{~ms}$, junto con las obtenidas experimentalmente para dos tipos de premezcla, denominados "high penetration" y "low penetration" [76]. La tendencia es muy similar, siendo las emisiones obtenidas muy cercanas a las experimentales con premezcla perfecta [77], mientras que las demás emisiones experimentales crecen al disminuir el nivel de premezclado. De hecho, la diferencia en emisiones respecto al caso de 
premezcla perfecta se podría usar para evaluar el nivel de premezcla, como sugieren Barnes y Mellor [78].

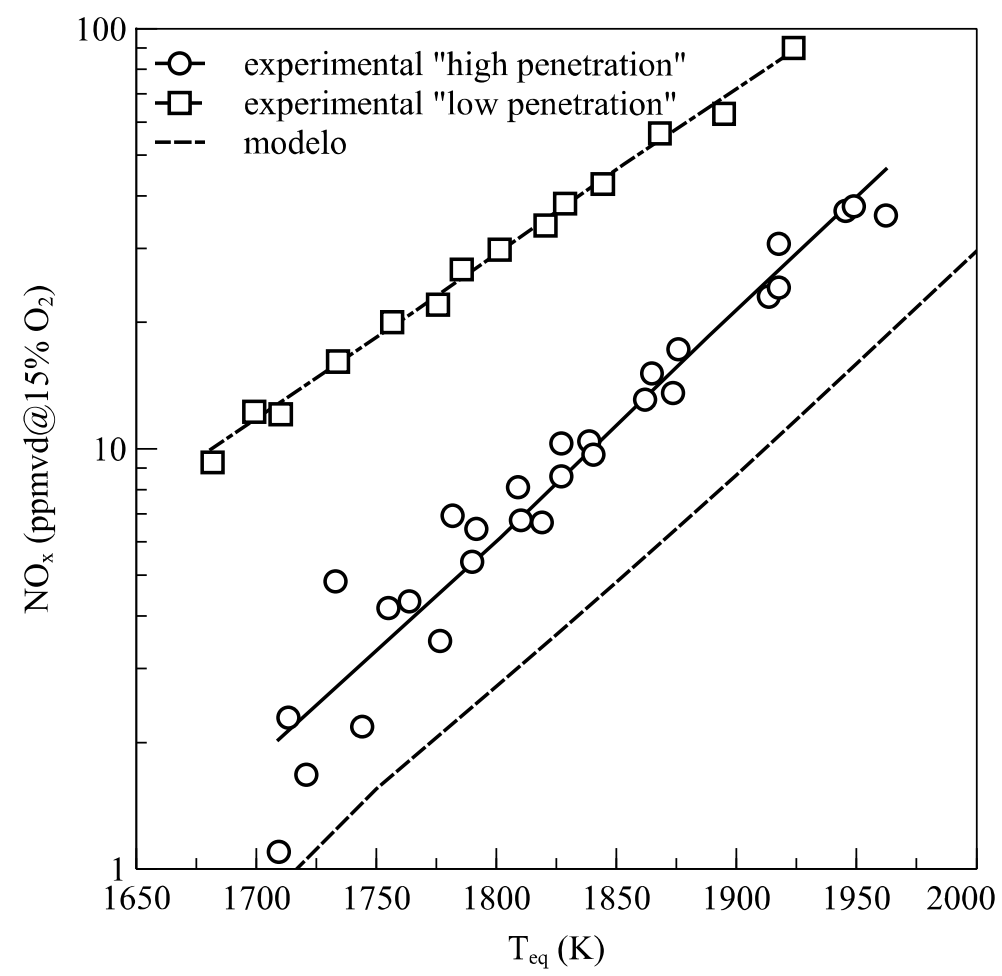

Figura 47. Comparación entre las emisiones de $\mathrm{NO}_{x}$ experimentales [76], y las obtenidas mediante el modelo.

Teniendo en cuenta la escasa información empleada para construir el modelo, este reproduce cualitativamente bien el comportamiento de la cámara de combustión elegida, con lo que se considera adecuado para establecer una comparación con un modelo funcionando con gas recirculado.

El comportamiento de este modelo usando $\mathrm{N}_{2}$ como diluyente se puede comparar con el de un modelo similar usando $\mathrm{CO}_{2}$, manteniendo constantes el resto de sus parámetros. El tamaño de la ZP se ajustará para dar el mismo gasto másico y la misma temperatura de equilibrio de apagado incipiente, y la carga base se definirá como la que proporciona la misma temperatura de equilibrio. Tanto el tamaño de la ZP como las relaciones de equivalencia variarán al cambiar de diluyente. 


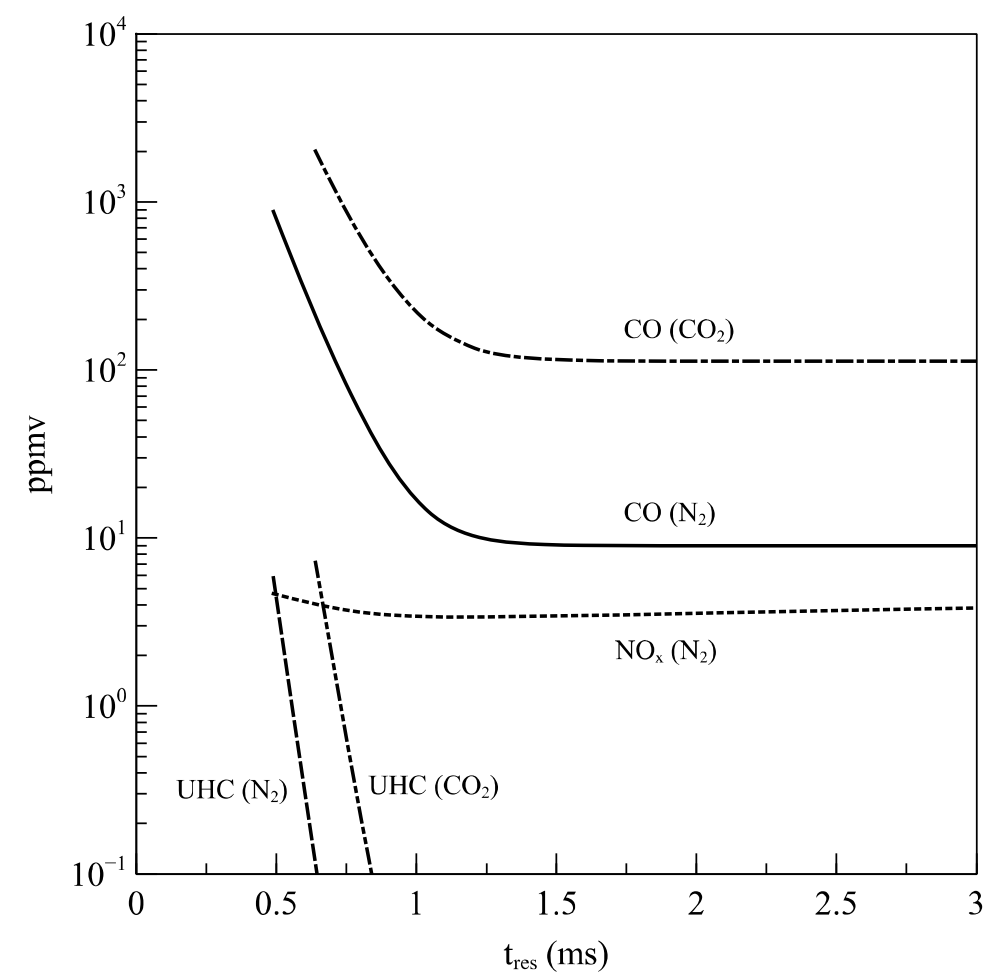

Figura 48. Evolución de los productos de combustión incompleta en la cámara para los diluyentes $\mathrm{CO}_{2}$ y $\mathrm{N}_{2}, d=d_{\text {aire }}=3.29$ y $T_{\text {eq,ZP }}=T_{\text {eq,BL }}=1820 \mathrm{~K}$.

La evolución de los productos de combustión incompleta monóxido de carbono $\mathrm{CO}$, hidrocarburos inquemados, UHC y óxidos de nitrógeno, $\mathrm{NO}_{\mathrm{x}}$, para la temperatura correspondiente a la de diseño de la cámara, $1820 \mathrm{~K}$, se presenta en la Figura 48. Los hidrocarburos presentes se consumen muy rápidamente, dando lugar al monóxido de carbono, que se reduce lentamente en la zona secundaria. Debido a la naturaleza asintótica de este proceso, el consumo de CO se considerará completado cuando se alcance un valor igual a 1.1 veces el correspondiente a la composición de equilibrio [70]. El tiempo necesario para alcanzar esta condición es similar para los dos diluyentes, aunque la concentración correspondiente es muy superior para el $\mathrm{CO}_{2}$, como se puede esperar de los resultados de equilibrio químico, que se van a mostrar a continuación. En posteriores casos también se empleará la longitud necesaria para lograr el consumo de $\mathrm{CO}$ en la cámara de combustión para comparar resultados, $L_{e q, C c}$. La concentración de $\mathrm{NO}_{\mathrm{x}}$ crece con el tiempo de residencia cuando hay nitrógeno presente, lo que concuerda con el comportamiento en cámaras de combustión reales. 


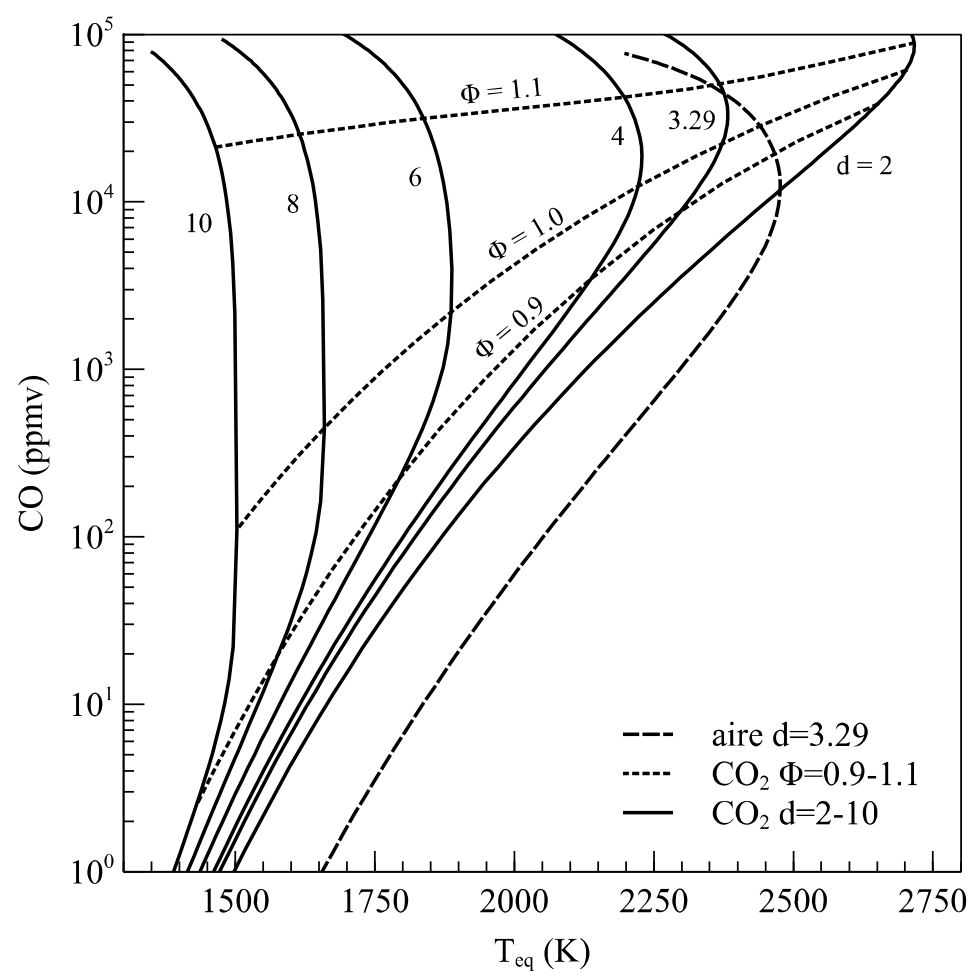

Figura 49. Monóxido de carbono en equilibrio como función de la temperatura, $\Phi$, y d para diluyente $\mathrm{CO}_{2}$ con $\mathrm{P}=30$ bar, la línea discontinua corresponde al aire $\left(N_{2}, d=3.29\right)$.

Como límite del proceso de consumo del CO, la Figura 49 muestra un mapa con la temperatura y la concentración de $\mathrm{CO}$ de equilibrio para $\mathrm{P}=30$ bar en función de $\Phi$ y $d$, para diluyente $\mathrm{CO}_{2}$ y usando $\mathrm{CH}_{4}$ como combustible; también se muestra la curva correspondiente al aire $\left(\mathrm{N}_{2}, d=3.29\right)$. Para conseguir bajos niveles de $\mathrm{CO}$ usando $\mathrm{CO}_{2}$ como diluyente hay que conseguir $\Phi<0.9$ y $T_{4 t}$ menor de $1700 \mathrm{~K}$. En cualquier caso, el CO de equilibrio será mucho mayor que el de combustión con aire. Los valores escogidos para el punto de diseño del ciclo dan aproximadamente $\mathrm{CO}_{e q}=16$ ppmv.

\subsection{Comportamiento de la zona de dilución.}

La distribución de dilución apropiada debería resultar de un estudio detallado de la forma de la carcasa y de los requisitos de refrigeración, algo que no se pretende en este estudio. En su lugar se pretende usar una distribución muy simple, en la que el número de parámetros requeridos para su definición sea el mínimo posible. Esto facilitará la comparación entre la oxicombustión y la combustión con aire. 

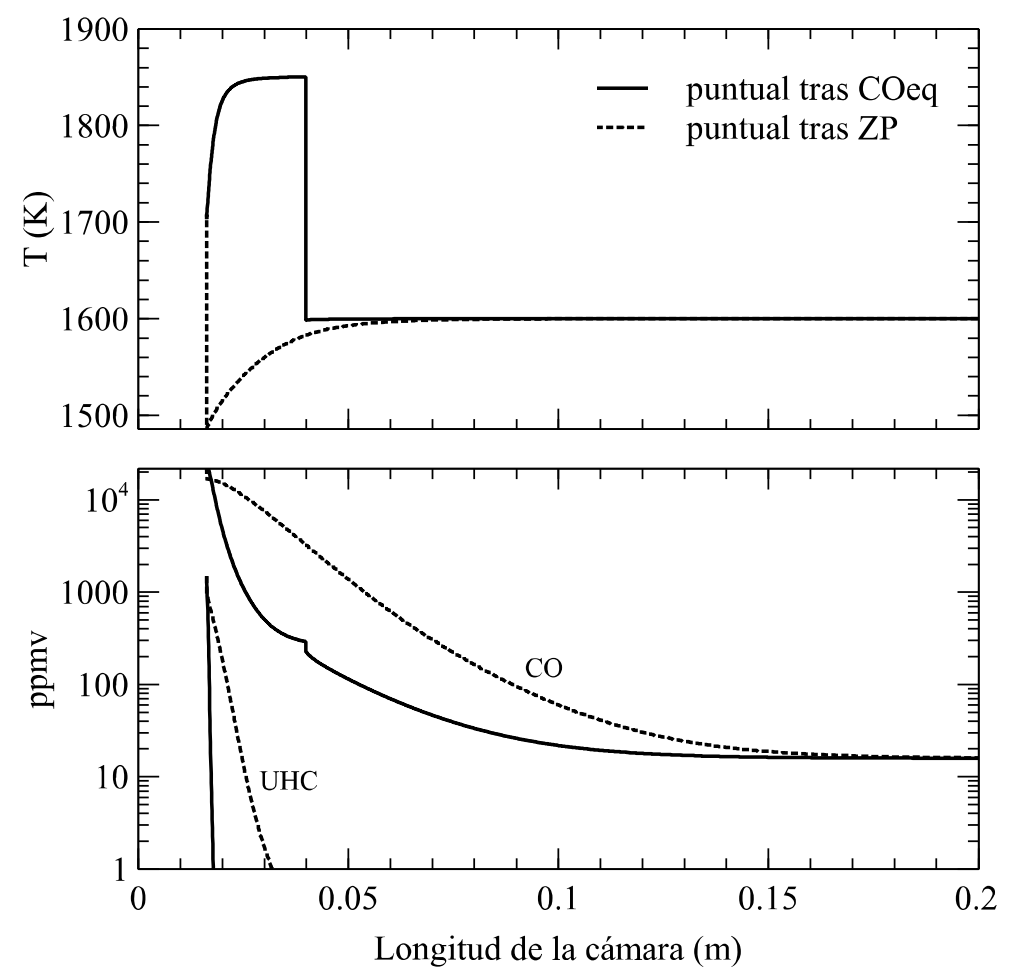

Figura 50. Evolución de la temperatura (superior) y la fracción volumétrica de CO y UHC (inferior) en la cámara del CCSC para inyección puntual del diluyente justo tras la ZP y tras alcanzar $\mathrm{CO}_{e q}$ en la $S Z, T_{e q, P Z}=1850 \mathrm{~K}, \Delta T_{L B O}=150 \mathrm{~K}$ y $M_{\text {ref }}=0.05$.

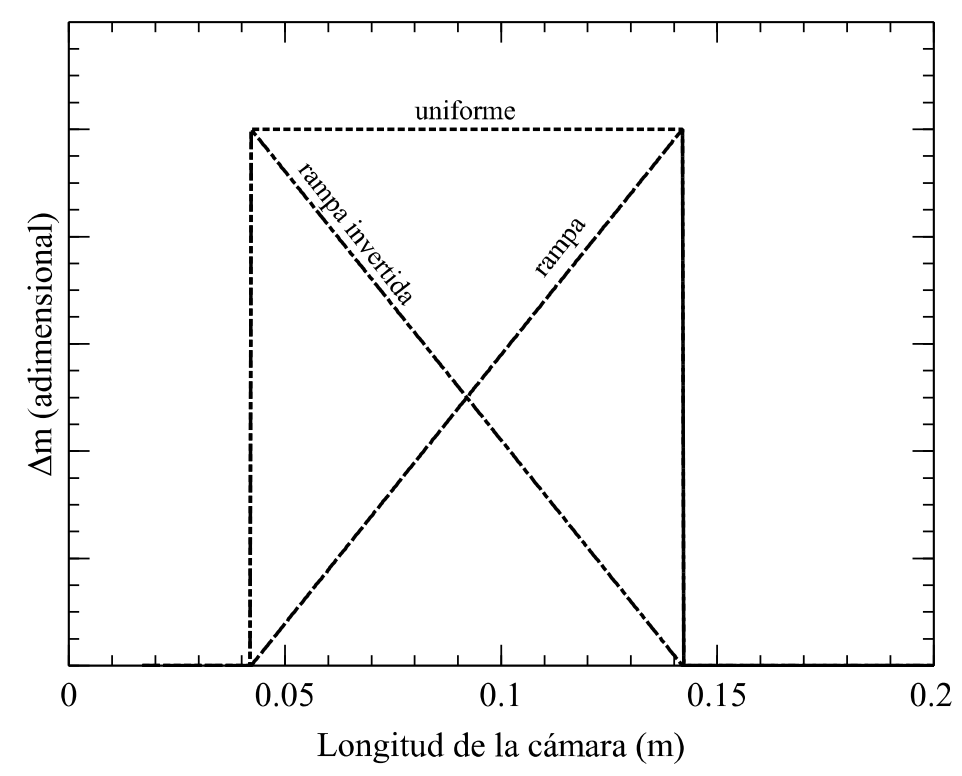

Figura 51. Gasto másico inyectado adimensionalizado con el valor máximo en cada caso, para las distribuciones uniforme, en forma de rampa y de rampa invertida, para el caso de la cámara del $C C S C$ con $T_{e q, P Z}=1850 \mathrm{~K}, \Delta T_{L B O}=50 \mathrm{~K}$ y $M_{\text {ref }}=0.05, \Delta L_{\text {dilución }}=0.1 \mathrm{~m}$.

La evolución de la combustión en la SZ sugiere dos puntos característicos para comenzar la dilución: la salida de la ZP, típica de configuraciones tipo 1, y la 
estación de la SZ donde se ha logrado el consumo de CO (1.1 veces el valor de equilibrio). La evolución de la temperatura y los contaminantes para inyección de todo el gasto másico disponible para dilución en estos dos puntos se presentan en la Figura 50. Resulta evidente que, al mezclar todo el gas restante con la corriente principal, la temperatura cae considerablemente, lo que perjudica especialmente el consumo de CO del caso con inyección tras la ZP, con lo que en los casos sucesivos siempre se comenzará la inyección tras el punto de $\mathrm{CO}_{e q}$ de la SZ.
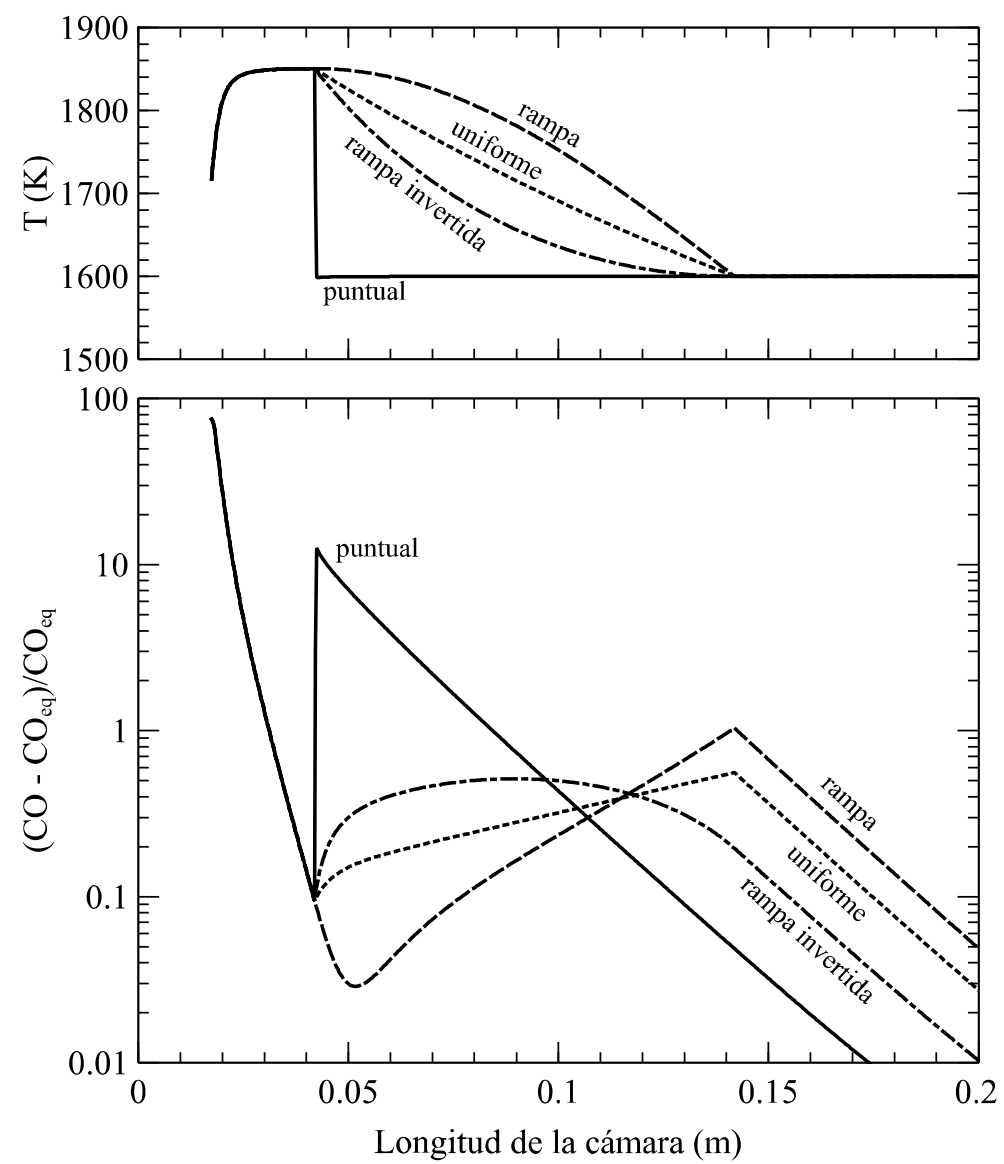

Figura 52. Evolución de la temperatura (superior) y la fracción de CO respecto a la de equilibrio (inferior) en la cámara del CCSC para distribución uniforme, en forma de rampa y de rampa invertida con, $\Delta L_{\text {dilución }}=0.1 \mathrm{~m}, T_{\text {eq, } P Z}=1850 \mathrm{~K}, \Delta T_{L B O}=50 \mathrm{~K}$ y $M_{\text {ref }}=0.05$.

Además del punto de inicio de la dilución, hay que seleccionar la forma y la longitud total de la distribución. Para ello se van a comparar tres distribuciones distintas, una distribución uniforme, una distribución en forma de rampa, creciente con la longitud, y una distribución en forma de rampa invertida, 
decreciente con la longitud. Para clarificar la nomenclatura, la Figura 51 muestra el gasto inyectado para el caso de la cámara del CCSC con $T_{\text {eq,PZ }}=1850$ $\mathrm{K}, \Delta T_{L B O}=50 \mathrm{~K}$ y $M_{\text {ref }}=0.05, \Delta L_{\text {dilución }}=0.1 \mathrm{~m}$. La evolución de la temperatura y el CO respecto al del equilibrio se muestran en Figura 52. Puede verse que la distribución que necesita una menor distancia para el consumo de $\mathrm{CO}$ es la de inyección puntual, seguida por la que más se le parece, la de forma de rampa invertida.
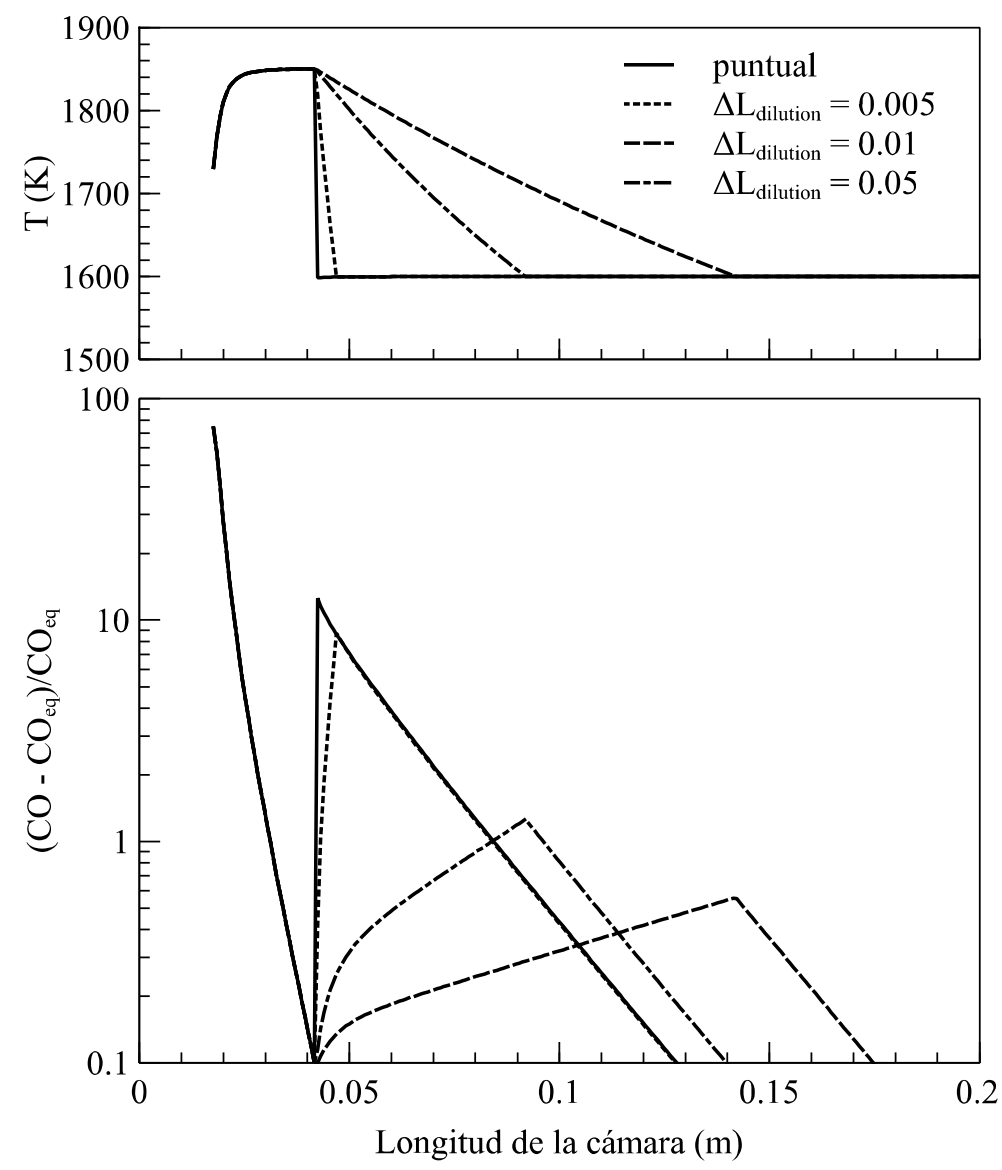

Figura 53. Evolución de la temperatura (superior) y la fracción de CO respecto a la de equilibrio (inferior) en la cámara del CCSC para distribuciones uniformes de diferente longitud, $\Delta L_{\text {dilución }} T_{\text {eq,PZ }}$ $=1850 \mathrm{~K}, \Delta T_{L B O}=50 \mathrm{~K}$ y $M_{\text {ref }}=0.05$.

El efecto de la longitud de la distribución se muestra en la Figura 53, donde se tienen la evolución de la temperatura y del $\mathrm{CO}$ respecto al del equilibrio en la cámara del CCSC para distribuciones uniformes de diferente longitud, $\Delta L_{\text {dilución, }}$ $\mathrm{T}_{\mathrm{eq}, \mathrm{PZ}}=1850 \mathrm{~K}, \Delta \mathrm{T}_{\mathrm{LBO}}=50 \mathrm{~K}$ y $\mathrm{M}_{\mathrm{ref}}=0.05$. De nuevo puede observarse que la evolución es más rápida cuanto más se parece a una inyección puntual. Sin 
embargo, la diferencia entre las longitudes obtenidas no es excesivamente grande, incluso para $\Delta L_{\text {dilución }}=0.1 \mathrm{~m}$, de modo que se va a utilizar como referencia en el resto del estudio la de inyección puntual en la estación inmediatamente posterior al consumo de CO en la SZ, teniendo en cuenta que lo que se pretende es comparar las longitudes mínimas de la cámara necesarias para combustión completa.

\subsection{Cámaras de combustión convencionales y del CCSC.}

Los resultados obtenidos anteriormente van a ser usados para estimar el comportamiento de cámaras de combustión diseñadas para las condiciones de entrada de turbinas de gas convencionales, $P=30$ bar, $T=720 \mathrm{~K}$, y cámaras de combustión diseñadas para las condiciones del CCSC. Los modelos de las cámaras de ambos casos se construirán para proporcionar el mismo gasto másico a la salida, $587.4 \mathrm{~kg} / \mathrm{s}$, y temperatura, $1600 \mathrm{~K}$, del punto de diseño del CCSC. En todos los casos se va a utilizar el esquema de dilución consistente en inyección puntual tras el consumo de CO en la SZ. Se va a asumir un número de Mach de referencia igual a 0.05 para estimar el tamaño. Restan $T_{\text {eq,ZP }}$ y $\Delta T_{L B O}$ como parámetros del modelo; una vez fijados ambos se pueden calcular el área de referencia, el volumen y la longitud de la ZP, y la longitud total necesaria para el consumo de CO como estimación de la longitud necesaria para la cámara.

\begin{tabular}{|c|c|c|c|c|c|c|c|c|c|c|c|c|}
\hline $\begin{array}{c}\mathrm{T}_{\text {eq,ZP }} \\
(\mathrm{K})\end{array}$ & $\begin{array}{c}\mathrm{V}_{\mathrm{ZP}} \\
\text { (m3) }\end{array}$ & $\begin{array}{c}\mathrm{m}_{\mathrm{ZP}} \\
(\mathrm{kg} / \mathrm{s})\end{array}$ & $\begin{array}{c}\mathrm{A}_{\mathrm{ref}} \\
(\mathrm{m} 2) \\
\end{array}$ & $\begin{array}{c}\begin{array}{c}\mathrm{U}_{\mathrm{ref}} \\
(\mathrm{m} / \mathrm{s})\end{array} \\
\end{array}$ & $\begin{array}{r}\mathrm{CO}_{\text {eq,ZP }} \\
(\mathrm{ppmv})\end{array}$ & $\begin{array}{l}\mathrm{t}_{\text {res,ZP }} \\
(\mathrm{ms}) \\
\end{array}$ & $\begin{array}{l}\mathrm{L}_{\mathrm{ZP}} \\
(\mathrm{m})\end{array}$ & $\begin{array}{r}\mathrm{CO}_{\text {eq, } \mathrm{CC}} \\
(\mathrm{ppmv})\end{array}$ & $\begin{array}{c}\mathrm{t}_{\mathrm{res}, \mathrm{ZP}+\mathrm{Zs}} \\
(\mathrm{ms})\end{array}$ & $\begin{array}{c}\mathrm{L}_{\mathrm{ZP}+\mathrm{ZS}} \\
(\mathrm{m})\end{array}$ & $\begin{array}{c}\mathrm{t}_{\text {res, }, \mathrm{CC}} \\
(\mathrm{ms})\end{array}$ & $\begin{array}{l}\mathrm{L}_{\mathrm{CC}} \\
(\mathrm{m}) \\
\end{array}$ \\
\hline 1700 & 0.068 & 523 & 1.17 & 20.7 & 54.0 & 1.30 & 0.06 & 15.9 & 2.06 & 0.11 & 2.94 & 0.16 \\
\hline 1800 & 0.031 & 471 & 1.06 & 20.8 & 161.0 & 0.62 & 0.03 & 15.9 & 1.04 & 0.06 & 2.21 & 0.13 \\
\hline 1900 & 0.016 & 426 & 0.96 & 20 & & 0.32 & 0.02 & & & 0.03 & 1.95 & 0.13 \\
\hline 2000 & 0.008 & 388 & 0.88 & 20.9 & 1015.0 & 0.18 & 0.01 & 15.9 & 0.33 & 0.02 & 1.87 & 0.14 \\
\hline
\end{tabular}

Tabla 25. Resultados para cámaras de combustión del CCSC con $\triangle T_{L B O}=100 \mathrm{~K}$. CC se refiere a las longitudes y tiempos de residencia de la cámara de combustión completa

En primer lugar se va a estudiar el efecto de $T_{e q, P Z}$ para $\Delta T_{L B O}=100 \mathrm{~K}$. Los resultados numéricos de la cámara del CCSC se muestran en la Tabla 25, mientras que los resultados para combustión con aire se muestran en la Tabla 26. 
4. Estudio de la cámara de combustión.

\begin{tabular}{|c|c|c|c|c|c|c|c|c|c|c|c|c|}
\hline $\begin{array}{c}\mathrm{T}_{\text {eq,ZP }} \\
(\mathrm{K})\end{array}$ & $\begin{array}{c}\mathrm{V}_{\mathrm{ZP}} \\
\text { (m3) }\end{array}$ & $\begin{array}{c}\mathrm{m}_{\mathrm{ZP}} \\
(\mathrm{kg} / \mathrm{s})\end{array}$ & $\begin{array}{c}\mathrm{A}_{\text {ref }} \\
(\mathrm{m} 2)\end{array}$ & $\begin{array}{c}\mathrm{U}_{\mathrm{ref}} \\
(\mathrm{m} / \mathrm{s})\end{array}$ & $\begin{array}{l}\mathrm{CO}_{\text {eq,ZP }} \\
(\mathrm{ppmv})\end{array}$ & $\begin{array}{l}\mathrm{t}_{\text {res,ZP }} \\
(\mathrm{ms}) \\
\end{array}$ & $\begin{array}{l}\mathrm{L}_{\mathrm{ZP}} \\
(\mathrm{m}) \\
\end{array}$ & $\begin{array}{l}\mathrm{CO}_{\mathrm{eq}, \mathrm{CC}} \\
(\mathrm{ppmv})\end{array}$ & $\begin{array}{c}\mathrm{t}_{\text {res, }, \mathrm{ZP}+\mathrm{ZS}} \\
(\mathrm{ms}) \\
\end{array}$ & $\begin{array}{c}\mathrm{L}_{\mathrm{ZP}+\mathrm{ZS}} \\
(\mathrm{m})\end{array}$ & $\begin{array}{c}t_{\text {res,CC }} \\
(\mathrm{ms}) \\
\end{array}$ & $\begin{array}{l}\mathrm{L}_{\mathrm{CC}} \\
(\mathrm{m}) \\
\end{array}$ \\
\hline 1700 & 0.057 & 519 & & & & & & & & & & \\
\hline 800 & 0024 & 464 & 1.20 & 26.0 & & 0.34 & 0.0 & & & 5 & 1.68 & 0.12 \\
\hline 900 & 4 & & & 26. & & & & & & & 1.49 & 0.12 \\
\hline 000 & 0.005 & 379 & 0.98 & 25.9 & 63.0 & 0.08 & 0.01 & 0.5 & 0.18 & 0.01 & 1.49 & 0.14 \\
\hline
\end{tabular}

Tabla 26. Resultados para cámaras de combustión con aire con $\triangle T_{L B O}=100 \mathrm{~K}$. CC se refiere a las longitudes y tiempos de residencia de la cámara de combustión completa

Los valores obtenidos para las longitudes son comparables a los que se pueden encontrar en la literatura. Incluso cerca de la pared (a unos $1100 \mathrm{~K}$ ), el CO puede alcanzar su valor de equilibrio en menos de $0.3 \mathrm{~m}$ [76]. Esta puede considerarse una longitud típica de cámaras de combustión de aerorreactores. La familia de turbinas de gas de producción de potencia de General Electric MS7000-9000 usa cámaras de combustión de tamaños entre 0.76 m (familia F) y 0.97 m (familia E)[79]. Sin embargo, este tipo de cámaras de combustión se diseñan para ser capaces de quemar muchos tipos distintos de combustible, lo que puede explicar que sean mucho mayores que los tamaños obtenidos, dado que el proceso puede estar dominado por los procesos de vaporización o ignición [78].

Los valores finales de CO obtenidos para la combustión con aire también son típicos. Para $T_{e q, P Z}<1800 \mathrm{~K}, \mathrm{CO}_{e q}$ es menor de 10 ppm, valor típico suministrado por los fabricantes de turbinas de gas [74]. Sin embargo, los valores dados para turbinas recientes son inferiores a $6 \mathrm{ppm}$, mientras que los resultados en ensayos son del orden de $2 \mathrm{ppm}$ [74]. Este último valor es aproximadamente el correspondiente a $\mathrm{CO}_{e q}$ para $1700 \mathrm{~K}$. Como era de esperar, los valores obtenidos para las condiciones del CCSC son muy superiores, debido al elevado contenido en $\mathrm{CO}_{2}$. En cualquier caso, el valor real de $\mathrm{CO}$ se espera que esté entre $\mathrm{CO}_{e q, P Z} \mathrm{y}$ $\mathrm{CO}_{e q, C C}$.

En la Figura 54 se muestra una comparación de los tiempos de residencia en cada zona de las cámaras de combustión con aire y oxicombustion, con $T_{\text {eq,PZ }}$ entre 1700 y $2000 \mathrm{~K}$ y $\Delta T_{L B O}=100 \mathrm{~K}$. Los resultados de la ZP y la ZS muestran 
una dependencia aproximadamente exponencial, decreciente con $T_{\text {eq,PZ, }}$ resultado que se puede atribuir a la dependencia exponencial con la temperatura de las reacciones químicas. Sin embargo, el tiempo de residencia en la ZD, sigue una exponencial creciente con $T_{\text {eq,PZ, }}$ lo que puede deberse a que el proceso de consumo de $\mathrm{CO}$ comienza en $\mathrm{CO}_{e q, P Z}$, que aumenta con $T_{e q, P Z}$, hasta alcanzar el mismo valor en todos los casos de $\mathrm{CO}_{e q}$ para $T_{4 t}$. Los tiempos de residencia necesarios para los casos con oxicombustión son mayores que los de combustión con aire en todas las zonas, especialmente en la ZP, siendo aproximadamente un $30 \%$ mayores para la cámara de combustión completa. También es de interés observar que los tiempos de residencia necesarios para el consumo de CO de las cámaras completas funcionando con aire presentan un mínimo cerca de 1950K, mientras que los de oxicombustión presentan un mínimo en $2000 \mathrm{~K}$.

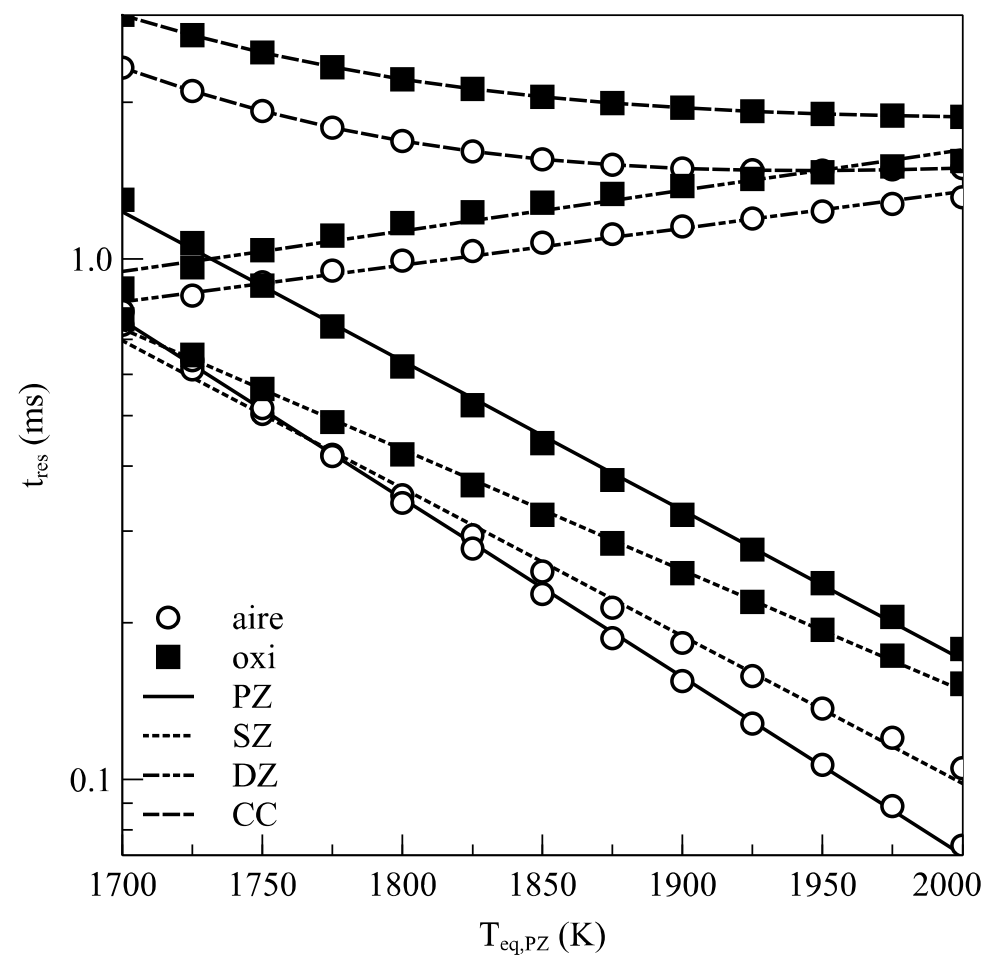

Figura 54. Variación con $T_{\text {eq,PZ }}$ del tiempo de residencia en cada zona para cámaras de combustión con aire y oxicombustión, con $\triangle T_{L B O}=100 \mathrm{~K}$. Los resultados de la $Z P$, ZS, y ZD han sido ajustados mediante una función exponencial.

En la Figura 55 se muestra una comparación similar para las longitudes de cada zona. Los resultados obtenidos para cada zona son cualitativamente similares a 
los de los tiempos de residencia, pero, mientras que los tamaños de las ZP de los casos con oxicombustión son mucho mayores que las de los casos con aire, los tamaños de las ZD de los casos con oxicombustión son ligeramente menores que los de los casos con aire, dando longitudes mucho más similares para la cámara completa. También puede verse que la $T_{\text {eq, } P Z}$ correspondiente a la longitud mínima de la cámara está cerca de $1850 \mathrm{~K}$ para ambos casos, siendo mucho menor que el valor correspondiente al mínimo de los tiempos de residencia.

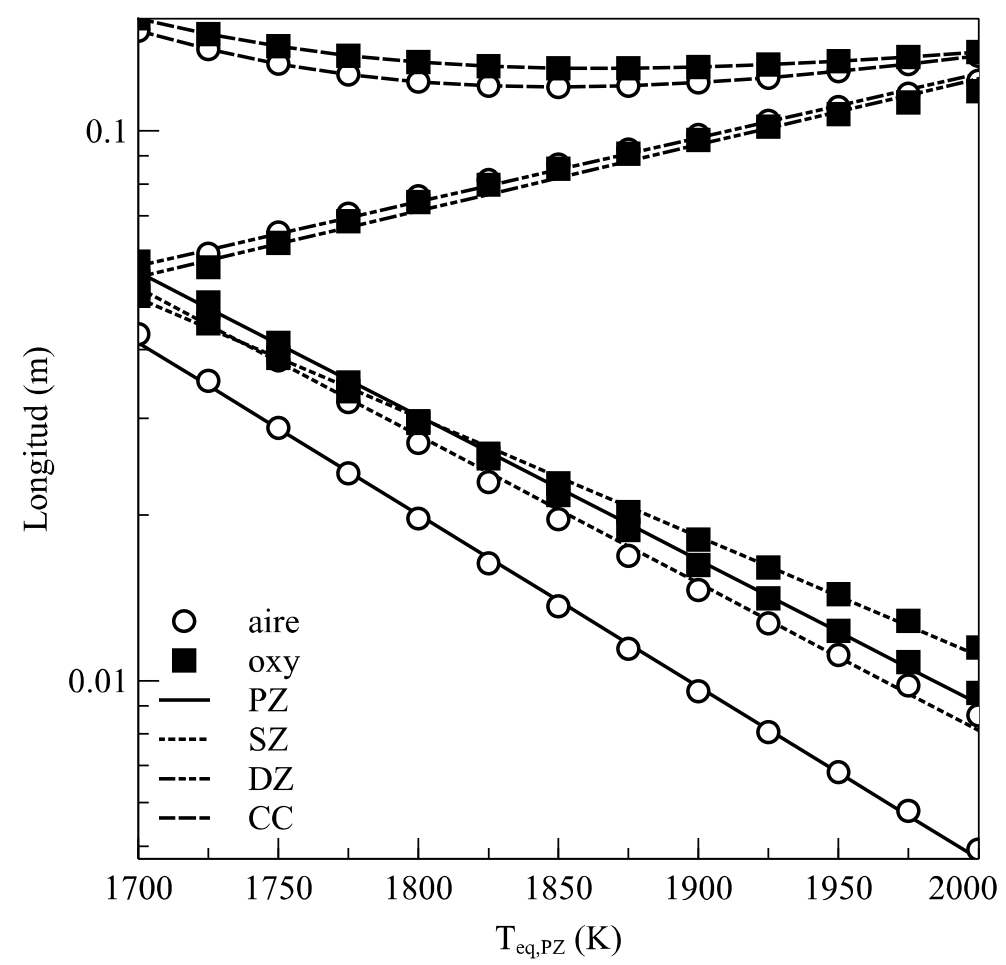

Figura 55. Variación con $T_{e q, P Z}$ de la longitud de cada zona para cámaras de combustión con aire y oxicombustión, con $\triangle T_{L B O}=100 \mathrm{~K}$. Los resultados de la $Z P$, ZS, y ZD han sido ajustados mediante una función exponencial.

\begin{tabular}{ccccccccccccc}
\hline $\begin{array}{c}\mathrm{T}_{\text {eq,ZP }} \\
(\mathrm{K})\end{array}$ & $\begin{array}{c}\mathrm{V}_{\mathrm{ZP}} \\
(\%)\end{array}$ & $\begin{array}{c}\mathrm{m}_{\mathrm{ZP}} \\
(\%)\end{array}$ & $\begin{array}{c}\mathrm{A}_{\text {ref }} \\
(\%)\end{array}$ & $\begin{array}{c}\mathrm{U}_{\text {ref }} \\
(\%)\end{array}$ & $\begin{array}{c}\mathrm{CO}_{\text {eq,ZP }} \\
(\%)\end{array}$ & $\begin{array}{c}\mathrm{t}_{\text {res,ZP }} \\
(\%)\end{array}$ & $\begin{array}{c}\mathrm{L}_{\mathrm{ZP}} \\
(\%)\end{array}$ & $\begin{array}{c}\mathrm{CO}_{\text {eq,CC }} \\
(\%)\end{array}$ & $\begin{array}{c}\mathrm{t}_{\text {res,ZP ZZ }} \\
(\%)\end{array}$ & $\begin{array}{c}\mathrm{L}_{\mathrm{ZP}+\mathrm{ZS}} \\
(\%)\end{array}$ & $\begin{array}{c}\mathrm{t}_{\text {res,CC }} \\
(\%)\end{array}$ & $\begin{array}{c}\mathrm{L}_{\mathrm{CC}} \\
(\%)\end{array}$ \\
\hline 1700 & 19 & 0.8 & -13 & -21 & 2600 & 65 & 50 & 3080 & 34 & 10 & 26 & 6.7 \\
\hline 1800 & 29 & 1.5 & -12 & -20 & 2200 & 82 & 50 & 3080 & 51 & 20 & 32 & 8.3 \\
\hline 1900 & 60 & 1.9 & -11 & -20 & 1836 & 100 & 100 & 3080 & 68 & 50 & 31 & 8.3 \\
\hline 2000 & 60 & 2.4 & -10 & -19 & 1511 & 125 & 0 & 3080 & 83 & 100 & 26 & 0.0 \\
\hline
\end{tabular}

Tabla 27. Variación de los resultados para cámaras de combustión del CCSC respecto a los resultados de cámaras de combustión con aire, con $\triangle \mathrm{T}_{L B O}=100 \mathrm{~K}$. CC se refiere a las longitudes y tiempos de residencia de la cámara de combustión completa 
La comparación del resto de resultados se presenta en la Tabla 27, donde se representa la diferencia entre el valor obtenido con gas recirculado y el valor correspondiente con aire. La menor velocidad de referencia se debe a la menor velocidad del sonido de este gas, y la menor área de referencia necesaria a su mayor densidad.

Los resultados anteriores han sido obtenidos para un valor fijo de $\Delta T_{L B O}=100 \mathrm{~K}$. Este parámetro tiene una gran influencia en el tamaño de la ZP, pero su influencia en el tamaño de la cámara completa no es tan grande, como se aprecia en la Figura 56. Estos resultados sugieren que el valor de $\Delta T_{L B O}$ escogido para el CCSC podría ser ligeramente mayor que el de cámaras de combustión de turbinas similares funcionando con aire sin tener un impacto excesivo en el tamaño de la cámara, y que $\Delta T_{L B O}=100 \mathrm{~K}$ puede usarse como un valor representativo.
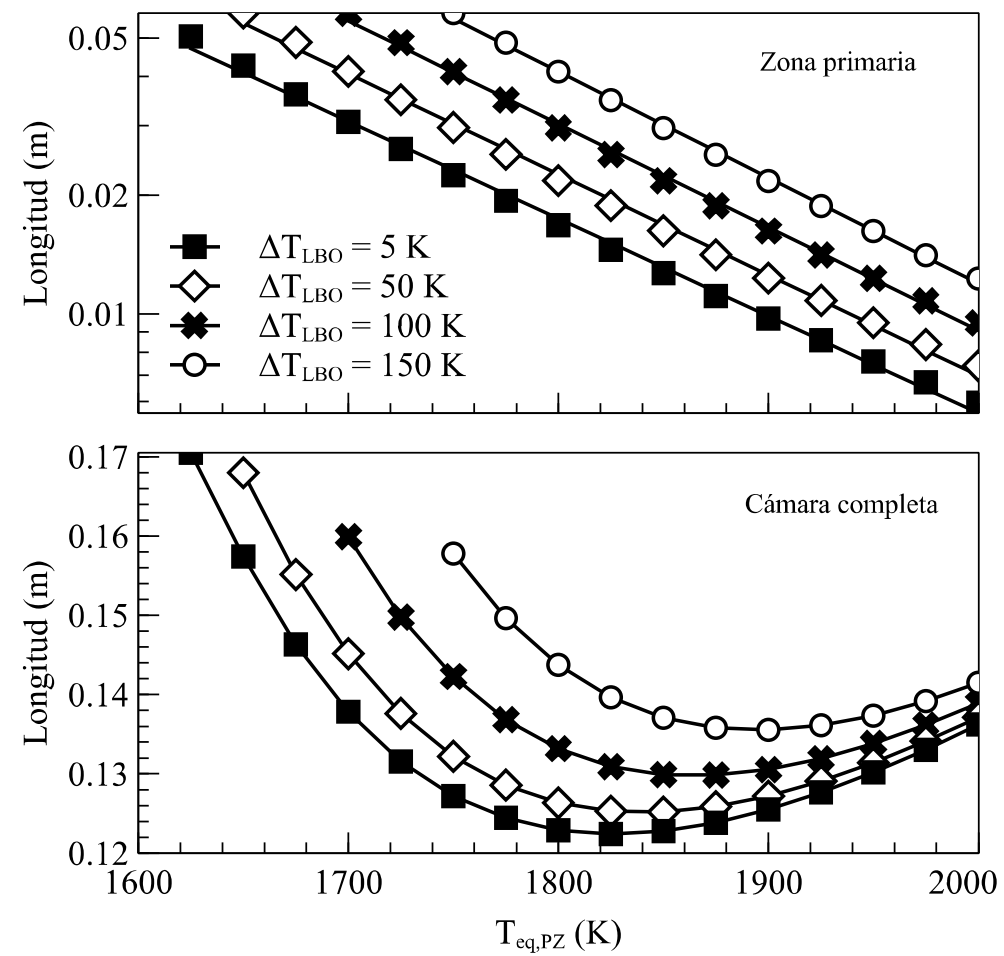

Figura 56. Variación con $T_{\text {eq,PZ }}$ y $\triangle T_{L B O}$ de la longitud de la $Z P$ (superior) y de la cámara de combustión completa (inferior) para oxicombustión. Los resultados de la ZP se han ajustado mediante una función exponencial. 


\subsection{Sensibilidad ante cambios en el punto de diseño del ciclo.}

En la línea del análisis de paramétrico llevado a cabo en el estudio del punto de diseño del ciclo, en esta sección se van a analizar de forma breve los efectos que puedan tener en el diseño de la cámara de combustión los cambios en los valores de diseño de la presión de combustión, $P_{3 t}$ y la temperatura de salida de la cámara, $T_{4 t}$.

Los efectos de cambios en la $P_{3 t}$ se muestran en la Figura 57. Como era de esperar, el incremento en la presión mejora el proceso de consumo de $\mathrm{CO}$, requiriendo valores menores de longitudes y tiempos de referencia, sucediendo lo contrario si $P_{3 t}$ disminuye. La posición de $T_{e q, Z P}$ para los puntos de mínima longitud y tiempo de referencia varía poco con la presión. Un efecto adicional no mostrado aquí es que el incremento de presión generará valores inferiores de $\mathrm{CO}_{e q}$ a la salida.
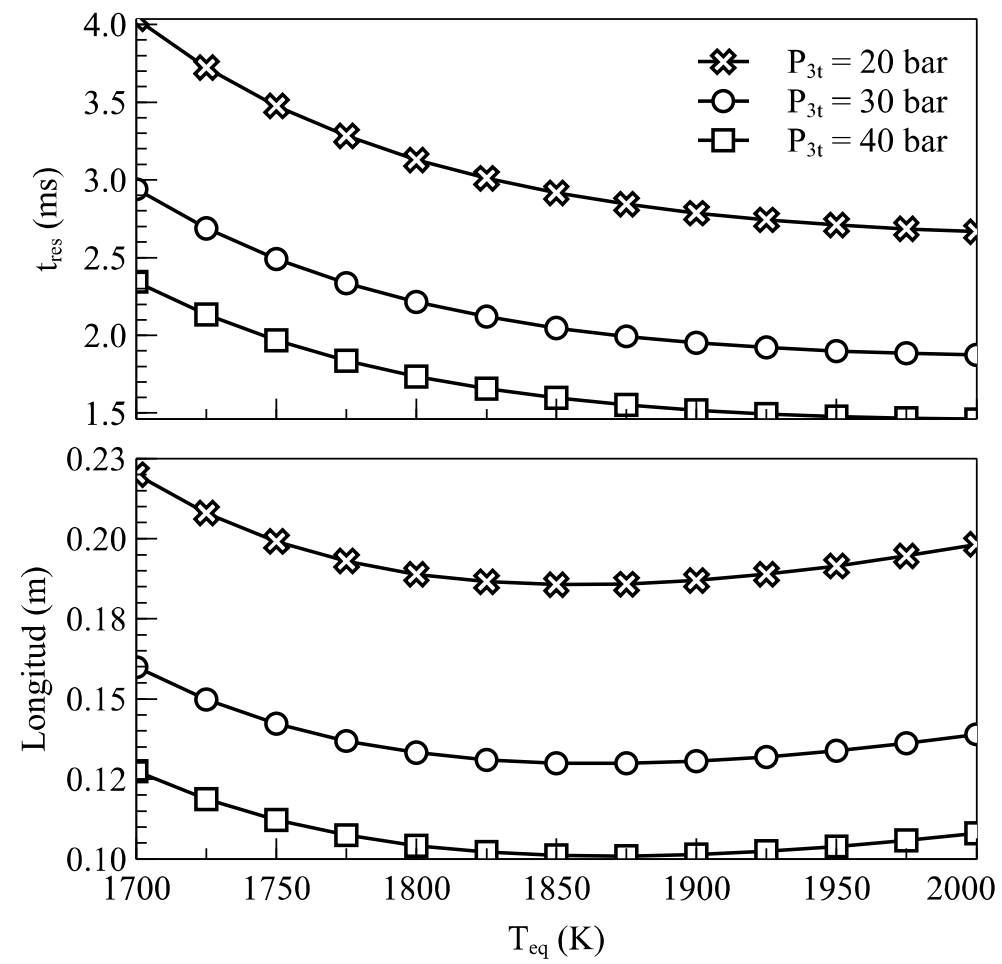

Figura 57. Variación con $T_{e q, Z P}$ y $P_{3 t}$ de los tiempos de residencia (superior) y longitudes (inferior) necesarios para el consumo de CO en la cámara de combustión completa del CCSC para $\triangle T_{L B O}=$ $100 \mathrm{~K}$ 


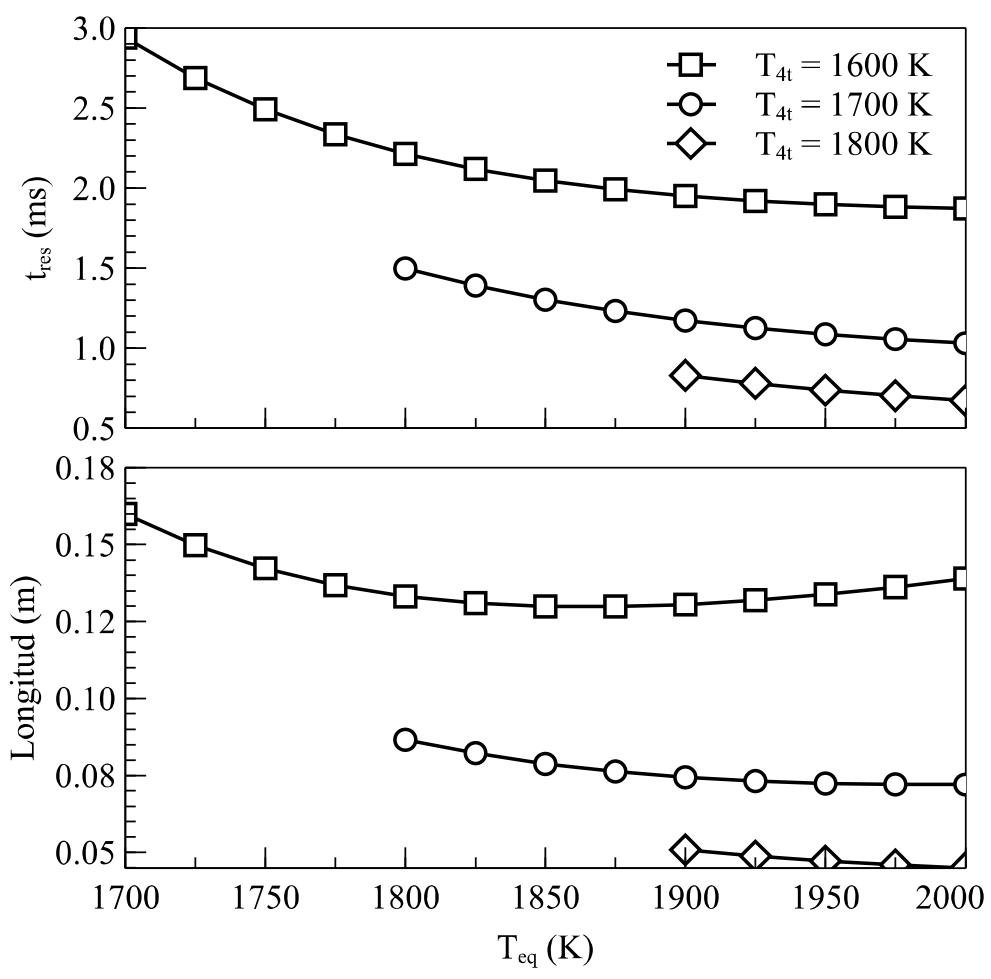

Figura 58. Variación con $T_{\text {eq,ZP }}$ y $T_{4 t}$ de los tiempos de residencia ( superior) y longitudes (inferior) necesarios para el consumo de CO en la cámara de combustión completa del CCSC para $\triangle T_{L B O}=$ $100 \mathrm{~K}$.

La Figura 58 muestra el efecto de incrementos en $T_{4 t}$. Los valores necesarios para el consumo de CO disminuyen considerablemente al aumentar $T_{4 t}$. La posición de $T_{e q, Z P}$ para alcanzar los mínimos de la longitud y del tiempo de residencia se desplazan considerablemente hacia valores superiores. 


\section{CONCLUSIONES.}

La presente tesis se ha centrado en el estudio preliminar de ciclos combinados semicerrados con captura de $\mathrm{CO}_{2}$ mediante oxicombustión de gas natural y $\mathrm{CO}_{2}$ como diluyente principal. Estos ciclos utilizan una configuración similar al ciclo combinado convencional, con un ciclo Brayton semicerrado, en el que se tiene un gas rico en $\mathrm{CO}_{2}$ como fluido de trabajo, en la cantidad necesaria para controlar el proceso de combustión y las condiciones de salida de la cámara. El oxidante se obtiene mediante una unidad de separación de aire criogénica, ASU (Air Separation Unit), que extrae el nitrógeno del aire, obteniendo un flujo de oxígeno de alta pureza ( $\geq 95 \%$ ), que se usa para quemar el combustible, un gas natural en este caso. Tras la combustión se separa el agua generada mediante condensación, la parte correspondiente de los gases restantes se comprime para su almacenamiento y el resto se recircula. Para mejorar la eficiencia global se utiliza un ciclo secundario de recuperación tipo Rankine, con agua como fluido de trabajo, dando lugar a un ciclo combinado semicerrado, CCSC.

Se ha perseguido el objetivo general de identificar las diferencias entre estos ciclos combinados y los que emplean turbinas de gas convencionales y las barreras que puedan existir a la hora del desarrollo/adaptación de componentes. Para ello se ha estudiado el ciclo en su punto de diseño, su comportamiento a carga parcial y su cámara de combustión como caso particular de adaptación de componentes, mediante técnicas características de estudio a nivel preliminar. Dado que es la parte que diferencia el CCSC de los ciclos combinados convencionales, los estudios se han centrado en el comportamiento del ciclo de gas semicerrado y su comparación con el comportamiento de ciclos abiertos funcionando con aire.

En primer lugar se ha estudiado la configuración del ciclo en su punto de diseño, mediante un modelo de simulación basado en componentes. Los cálculos se han 
realizado mediante un código propio del departamento de Mecánica de Fluidos y Propulsión Aeroespacial de la ETSIA, denominado Sistema Flexible de Simulación de Aerorreactores, SFSA, usando la composición real de los gases con la suposición de composición de equilibrio en cada estación para el ciclo de gas, y un modelo estándar en la industria para las propiedades del ciclo de vapor.

Para obtener los parámetros necesarios para definir cada componente se han estudiado los parámetros de componentes de turbinas de gas convencionales disponibles en la literatura científica, se han realizado simulaciones de turbinas convencionales para las que se tienen resultados de actuaciones y se han comparado con los parámetros usados en la literatura científica para ciclos semicerrados similares al objeto de estudio de esta tesis. Se ha asumido que se va a conseguir el mismo nivel de eficiencia en los componentes diseñados para los ciclos semicerrados que el de los componentes de ciclos de aire convencionales, con un rendimiento iséntropico igual a 0.87 en los compresores y un rendimiento isentrópico igual a 0.89 en las turbinas. Aunque los valores utilizados pueden no ser completamente representativos del estado del arte de las turbinas actuales, cumplen el propósito de permitir la comparación entre los ciclos combinados semicerrados y los convencionales.

Como parte del proceso de modelado se ha realizado un estudio simplificado del balance de gasto másico en el ciclo, necesario para obtener la composición del gas recirculado y para el análisis cualitativo de los consumos del sistema de producción de oxidante, ASU, y de captura de $\mathrm{CO}_{2}$ para su almacenamiento, CCS $\left(\mathrm{CO}_{2}\right.$ Capture System). El autor de esta tesis no tiene conocimiento de estudios similares para este ciclo en la literatura.

A continuación, y como paso previo al análisis paramétrico del ciclo, se han utilizado métodos simplificados para analizar el efecto del cambio de composición en el rendimiento y la potencia específica de un ciclo de gas. Aunque este tipo de análisis aplicado a ciclos de gas se puede encontrar en otros autores, en este caso se ha incluido la influencia de la ASU y del CCS. El análisis 
simplificado ha mostrado que el rendimiento máximo del ciclo de gas no depende del gas en el modelo utilizado, aunque la relación de presiones a la que se produce dicho máximo sí puede variar considerablemente. También ha mostrado que los consumos de la ASU y del CCS tienen un efecto muy significativo en el rendimiento del ciclo.

El análisis paramétrico del ciclo ha mostrado el impacto de la relación de compresión, la temperatura máxima del ciclo y la temperatura de recirculación en el modelo completo del CCSC, comparándolo con ciclos combinados convencionales, con los siguientes resultados:

La relación de compresión que maximiza el rendimiento del CCSC es mayor que la correspondiente al ciclo combinado convencional, aunque su variación es muy pequeña en un amplio rango de relaciones de presiones. Para una temperatura máxima del ciclo de $1600 \mathrm{~K}$, el máximo del rendimiento del sistema corresponde una relación de compresión de aproximadamente 40 con una ligera caída en el rango de presiones entre 30 y 50; estos valores están claramente dentro del rango de las turbinas actuales, permitiendo una amplia gama de diseño sin caídas importantes en el rendimiento. Las temperaturas de salida de los compresores del ciclo semicerrado y los valores de la temperatura a la entrada de la turbina de vapor permanecen en valores aceptables en este rango. Un aspecto revelado por este análisis que el autor no ha visto en la literatura es que el punto de acercamiento mínimo entre el ciclo semicerrado y el ciclo de vapor en el generador de vapor se desplaza hacia la entrada al evaporador del lado vapor para relaciones de presión bajas, penalizando el rendimiento global.

El valor de la eficiencia máxima del CCSC es ligeramente superior a la del ciclo combinado convencional si no se incluyen los consumos de la ASU y del CCS, pasando a ser ligeramente inferior cuando se incluyen.

La potencia específica del CCSC es superior a la del ciclo convencional, y su disminución con la relación de presiones es mucho menor, por lo que se espera 
un sistema más pequeño para una potencia dada, posiblemente con un coste menor.

$\mathrm{El}$ aumento de la temperatura máxima del ciclo acompañado por un incremento del gasto másico de refrigeración aumenta tanto el rendimiento máximo como la potencia específica para los casos estudiados.

Un aspecto poco estudiado por otros autores, y que se muestra en esta tesis, es que es necesario tener en cuenta la composición del gas recirculado para un análisis correcto de las actuaciones del ciclo semicerrado. Esto se aprecia en el efecto en el rendimiento de la disminución de la temperatura mínima del ciclo, que depende de las hipótesis que se hagan sobre su efecto en dicha composición.

Se ha seleccionado un posible punto de diseño con una relación de presiones de 30 y una temperatura máxima del ciclo de $1600 \mathrm{~K}$ para el análisis a carga parcial y el de la cámara de combustión. Los resultados de este punto se han acompañado con un estudio de sensibilidad que puede ser útil en estudios posteriores.

Como conclusión final del análisis de ciclo en su punto de diseño se puede concluir que el ciclo semicerrado no conlleva dificultades especiales respecto a los ciclos convencionales con aire, salvo por la penalización impuesta por la ASU y el CCS. También se puede concluir que cualquier análisis de este tipo de ciclos requiere la inclusión del ciclo secundario, aunque sea de una forma simplificada.

A continuación se ha estudiado el comportamiento del CCSC a carga parcial. Para ello se ha utilizado la misma técnica de modelado basado en componentes y el mismo código SFSA, aunque los modelos de los componentes y del sistema fuera de su punto de diseño son diferentes. En concreto, el compresor de gas recirculado y las turbinas requieren de mapas de actuaciones, que no se han podido encontrar en la literatura. Este problema se ha resuelto utilizando mapas de compresores y turbinas de ciclos convencionales, adaptándolos a su funcionamiento con gas recirculado mediante técnicas basadas en análisis 
dimensional. Como estas técnicas se basan en intentar que los números de Mach axial y circunferencial sean similares en el funcionamiento con aire y con gas recirculado, predicen gastos másicos superiores y velocidades de giro inferiores cuando funcionan con gas recirculado. La mayor incertidumbre está en la estimación de la eficiencia, para la que se ha asumido que se mantiene la eficiencia politrópica. Pese a que existe un estudio que apoya esta suposición para compresores centrífugos de pequeño gasto, su aplicabilidad a las turbomáquinas de este ciclo solo se podrá evaluar mediante estudios experimentales a mayor escala.

En el modelo de simulación del comportamiento a carga parcial de turbinas convencionales se suele contar con dos parámetros de control, una regulación del gasto másico de gas mediante álabes guía de geometría variable a la entrada, y varias etapas de estatores de geometría variable en el compresor VIGV/VSV (Variable Inlet Guide Vanes/Variable Stator Vanes), y una regulación de la temperatura a la salida de la cámara de combustión mediante el control del gasto de combustible. Ambos parámetros se pueden regular para conseguir variar la potencia del ciclo de gas manteniendo iguales las vueltas de la turbina de baja y la turbina de potencia, dando lugar a configuraciones de turbina monoeje, HD (Heavy Duty), o variando las vueltas de la turbina de alta de forma separada de las de baja, que se suelen mantener constantes, dando lugar a configuraciones bieje o aeroderivadas, AD.

Los parámetros de control se suelen combinar para dar lugar a distintas leyes de control. Es importante tener en cuenta que el control mediante VIGV/VSV puede no permitir más que un cierto rango de control del gasto, por algunas leyes teóricamente posibles no lo serán en la práctica. Para configuraciones $\mathrm{AD}$ se ha considerado control de $T_{4 t}$ únicamente, configuración denominada $\mathrm{AD} T 4 \mathrm{t}$, control manteniendo constante la temperatura a la salida de la turbina de baja AD T5t, y una ley mixta que mantiene T5t constante para potencias altas y luego regula en $T_{4 t}$, denominada AD MIX. Para configuraciones HD se han definido 
una ley manteniendo las vueltas del eje constantes, HD N, una ley controlando únicamente $T_{4 t}, \mathrm{HD} \mathrm{T} 4 \mathrm{t}$, y una ley mixta que mantiene vueltas constantes para potencias altas y luego pasa a controlar $T_{4 t}$.

Esta tesis ha introducido como novedad para este tipo de ciclos, dentro de los conocimientos del autor, el uso de un parámetro de control adicional, la presión base del ciclo. Este tipo de control se puede aplicar a turbinas de gas de ciclo cerrado, mediante un sistema de almacenamiento de gas que permite regular el gasto másico disponible, variando la presión mínima del ciclo. De esta forma se puede alterar el comportamiento de la turbomaquinaria, permitiendo que esta opere en las mismas condiciones adimensionales pese a la variación en la potencia producida. Este grado de control adicional se puede utilizar junto al de gasto másico de combustible para una configuración monoeje con vueltas constantes, dando lugar a la ley denominada HD P0.

Teniendo en cuenta su considerable influencia en el comportamiento del ciclo en su punto de diseño, también se ha modelado el comportamiento a carga parcial del ciclo de vapor, permitiendo que varíe la presión máxima para adaptarse a las condiciones del ciclo de gas.

El comportamiento de la turbina de gas del CCSC se ha estudiado de manera detallada para cada ley de control, procediendo después a su comparación, centrada en el rendimiento del sistema completo. Como se esperaba por su operación a condiciones adimensionales aproximadamente constantes, la ley HD $\mathrm{N}$ mantiene un rendimiento muy alto en todo el rango analizado. A continuación se tienen las leyes HD N y AD T5t, aunque los límites en el control VIGV/VSV podrían requerir el uso de las correspondientes HD MIX y AD MIX. El rendimiento de estas últimas es muy similar, cayendo hasta un valor mayor al 90\% del de diseño para un 50\% de la potencia original del ciclo de gas, mientras que la HD P0 solo baja hasta el 99\%. Los parámetros del ciclo de vapor en los tres casos permanecen en valores aceptables, aunque el acercamiento entre los ciclos cae más para la ley HD P0 
Hay otros factores a tener en cuenta en la ley HD P0, que se dejan para estudios posteriores. El más importante es que requiere la instalación y análisis detallado del sistema de almacenamiento de gas, que debería ir acompañado del análisis del impacto de los posibles cambios en la composición del gas recirculado y su impacto en la turbomaquinaria. También habría que modelar con más detalle el ciclo de vapor para descartar posibles problemas derivados de la reducción del acercamiento de ambos ciclos en el generador de vapor.

La conclusión final del estudio del comportamiento del ciclo a carga parcial es que el sistema de control de presión base propuesto en esta tesis podría proporcionar una ventaja respecto a las leyes de control convencionales, con las salvedades anteriormente mencionadas. Además, las leyes de control convencionales AD MIX y HD MIX proporcionan rendimientos similares, por lo que la selección de la configuración y/o ley de control dependerá de otras consideraciones como el tiempo esperado de operación a carga parcial, el tamaño del sistema (las turbinas de gas de gran potencia como las de este estudio son monoeje), el comportamiento ante pérdidas de carga, y el análisis detallado de los componentes.

Como caso particular de adaptación de componentes, se ha estudiado el comportamiento de la cámara de combustión del ciclo semicerrado. Para ello se ha construido un modelo simplificado de la cámara usando redes de reactores químicos, consistente en un reactor de flujo continuo, bien agitado, WSR (Well Stirred Reactor), al que después se ha acoplado un reactor tipo Plug Flow, PFR (Plug Flow Reactor). Para realizar el estudio, se ha utilizado al paquete de cinética química Cantera [75], junto con el esquema de cinética química GriMech 3.0 [58].

La cámara de combustión se ha dividido en tres zonas:

- La zona primaria, ZP, donde la llama está anclada mediante recirculación, está representada mediante el WSR. En dicha zona se inyectan el gasto 
másico de combustible y de la ASU correspondientes al punto de diseño del ciclo, junto con una fracción del gasto másico de gas recirculado disponible a la entrada de la cámara. El estudio del comportamiento de esta zona ha llevado al uso de la temperatura de equilibrio en la ZP, $T_{\text {eq,ZP }}$, como una variable de diseño, fijando la fracción de aire/gas recirculado que hay que mezclar con el diluyente. El volumen de esta zona se ha fijado mediante una temperatura de apagado incipiente $T_{\text {eq,LBo }}$, siendo el margen de estabilidad, $\triangle T_{L B O}$, la diferencia entre ambas.

- La zona secundaria, ZS, es la parte del PFR situada entre la salida del WSR y el inicio de la inyección del gas recirculado restante. La evolución de la oxicombustión en esta zona se ha comparado con la combustión con aire utilizando una cámara de combustión descrita por Snyder et al [76], cuyos resultados experimentales se han reproducido de forma cualitativamente correcta mediante el modelo. El proceso de combustión puede describirse cualitativamente como un consumo muy rápido del combustible para producir monóxido de carbono, que se consume lentamente hasta alcanzar el equilibrio. Precisamente la evolución del CO hasta el equilibrio es la variable estudiada para definir la longitud necesaria de las cámaras de combustión. Como este proceso es asintótico, el consumo de $\mathrm{CO}$ se ha considerado completado cuando se ha alcanzado un valor igual a 1.1 veces el de la composición de equilibrio. El CO de equilibrio para oxicombustión es muy superior al de combustión con aire, debido a la presencia de mucho $\mathrm{CO}_{2}$ en el gas recirculado. Una consideración importante es que el $\mathrm{CO}$ de equilibrio crece mucho con la temperatura, lo que podría resultar un problema si se usan valores muy grandes de $T_{4 t}$.

- La zona de dilución, ZD, es la parte restante del PFR, donde se inyecta el gas recirculado no inyectado en la zona primaria, donde idealmente se alcanzan las condiciones de equilibrio correspondientes a la salida de la cámara de combustión. El comportamiento del modelo para distintas 
distribuciones de inyección del gas ha llevado a la selección de la distribución que lleva a obtener la mínima longitud en la cámara, la inyección puntual de todo el gasto recirculado adicional tras alcanzar el consumo de CO en la zona secundaria.

Como el gas recirculado contiene cierta cantidad de oxidante, la relación de equivalencia real en la cámara puede ser bastante diferente de la especificada por la relación de gasto másico de la ASU y combustible, un aspecto no tratado en la literatura, dentro de lo conocido por el autor. Se ha obtenido la relación entre ambas relaciones de equivalencia, mostrando que la relación de equivalencia real en la cámara es muy inferior a la dada en el ciclo. También se ha creado un modelo auxiliar de la cámara de combustión, en el que se ha separado el gasto másico de oxidante del gas recirculado, creando un modelo con un diluyente, un oxidante y un combustible. Este modelo permite una comparación más sencilla con la combustión con aire en algunos casos, y se ha usado en los estudios del comportamiento de cada zona. La combustión con aire puede considerarse un caso especial de oxicombustión diluida, utilizando $\mathrm{N}_{2}$ como diluyente y una relación de gastos másicos entre el diluyente y el oxidante igual a 3.29 .

Una vez seleccionados los parámetros de diseño del modelo y la distribución de dilución, se han construido y comparado modelos para la cámara de combustión del CCSC y para cámaras de combustión con aire. Esta comparación ha mostrado que los tiempos de residencia esperados para el consumo de $\mathrm{CO}$ en cámaras con oxicombustión son aproximadamente un $30 \%$ superiores a los de cámaras de combustión con aire, aunque las diferencias entre las longitudes asociadas son mucho menores. A cambio, las áreas de referencia necesarias para el paso del mismo gasto másico han resultados ser un $10 \%$ inferiores para el caso con oxicombustión. Se espera que una configuración similar a las de cámaras de combustión de turbinas de gas de bajas emisiones actuales, con temperaturas de equilibrio en la zona primaria moderadas (cerca de $1850 \mathrm{~K}$ ) genere 
concentraciones de $\mathrm{CO}$ a la salida cerca de la de equilibrio con un tamaño comparable a las cámaras de combustión de turbinas de gas funcionando con aire.

También se ha realizado un análisis de sensibilidad del diseño de la cámara de combustión a la variación de los parámetros que se han usado para definir el ciclo, en el que se ha mostrado que las longitudes y los tiempos de residencia se reducirían si se aumenta la presión de la cámara y la temperatura a la salida de la cámara.

La conclusión fundamental del estudio de la cámara de combustión del CCSC es que probablemente sea necesario diseñarlas específicamente para este ciclo, dadas las diferencias en las características de estabilidad y tamaño de cada zona, pero no se ha encontrado ningún obstáculo fundamental que pueda impedir dicho diseño, siendo de esperar la obtención de tamaños similares a las de cámaras de combustión con aire. 


\section{BIBLIOGRAFÍA.}

[1] Y. Shao, D. Colomb, G. Brown, "Natural Gas Combined Cycle Power Plant with CO2 Capture", Energy Convers. Mgmt Vol 36 No 12, pp 1115-1128, 1995.

[2] I. Ulizar, P. Pilidis, "A semiclosed-cycle gas turbine with carbon dioxideargon as working fluid". Trans. ASME J. Eng. Gas Turb. Power, 1997, 119(7), 612-616.

[3] I. Ulizar, P. Pilidis, "Design of A Semiclosed Cycle Turbine With Carbon Dioxide-Argon Working Fluid", Trans. ASME J. Eng. Gas Turb. Power, 1998, 120(4), 330-335.

[4] I. Ulizar, P. Pilidis, "Handling of A Semiclosed Cycle GT With A Carbon Dioxide-Argon Working Fluid", Trans. ASME J. Eng. Gas Turb. Power, 2000, 122(7), 437-441.

[5] P. Mathieu, R. Nihart, "Zero emission MATIANT cycle", Trans. ASME J. Eng. Gas Turb. Power, 1999, 121(1), 116-120.

[6] K. Pronske, L. Trowsdale, S. Macadam, F. Viteri, F. Bevc, D. Horazak "An overview of turbine and combustor development for coal-based oxy-singas systems", Proceedings of the ASME turbo expo conference 2006, Barcelona. Paper no. GT-2006-90816.

[7] J. Martinez-Frias, S. M. Aceves, J. R. Smith, H. Brandt, "Thermodynamic analysis of zero atmospheric emissions power plants", Trans. ASME J. Eng. Gas Turb. Power, 2004, 126(1), 2-8.

[8] C. W. Hustad, I. Tronstad, R. E. Anderson, K. Pronske, F. Viteri, "Optimization of thermodynamically efficient nominal $40 \mathrm{mw}$ zero emission pilot and demonstration power plant in Norway", Proceedings of the ASME Turbo Expo conference 2005. 
Bibliografía.

[9] H. Jericha, E. Göttlich, W. Sanz, F. Heitmeir, "Design optimisation of the Graz prototype plant", Proceedings of the ASME turbo expo conference 2003, Atlanta, USA. Paper no. GT-2003-38120.

[10] H. Jericha, E. Göttlich, W. Sanz, F. Heitmeir, "Design Optimisation of the Graz Cycle Prototype Plant", ASME Journal of Engineering for Gas Turbines and Power, Vol. 126, pp. 733-740, October 2004.

[11] W. Sanz, H. Jericha, B. Bauer, E. Göttlich, "Qualitative and quantitative comparison of two promising oxy-fuel power cycles for $\mathrm{CO} 2$ capture", Proceedings of the ASME turbo expo conference 2007. paper no. GT200727375.

[12] T. Griffin, S. G. Sundkvist, A. Knut, T Brunn, "Advanced Zero Emissions Gas Turbine Power Plant", J. Eng. Gas Turbines Power 127, 81 (2005)

[13] S. Consonni, G. Lozza, G. Pelliccia, S. Rossini, F. Saviano, "ChemicalLooping Combustion for Combined Cycles With CO2 Capture", J. Eng. Gas Turbines Power 128, 525 (2006)

[14] H. K. Kvamsdal, K. Jordal, O. Bolland, "A quantitative comparison of gas turbine cycles with CO2 capture", Energy, 2007, 32, 10-24.

[15] J. Davison, K. Thambimuthu, "An overview of thecnologies and cost of carbon dioxide capture in power generation" Proc. IMechE, Part A: J. Power and Energy, 2006, 223, 315-325. DOI: 10.1243/09576509JPE625.

[16] T. S. Kim, S. H. Hwang, "Part load performance analysis of recuperated gas turbines considering engine configuration and operation strategy", Energy, 2006, 31, 260-277.

[17] A. L. Polyzakis, C. Koroneos, G. Xydis, "Optimum gas turbine cycle for combined cycle power plant", Energy Conversion and Management, 2008, 49(4), 551-563. 
Bibliografía.

[18] T. L. Ragland, "Industrial Gas Turbine Performance Uprates: Tips, Tricks, and Traps", Trans. ASME J. Eng. Gas Turb. Power, J. Eng. Gas Turbines Power, 1998, 120(4), 727-734.

[19] F. Haglind, "Variable geometry gas turbines for improving the part-load performance of marine combined cycles - Gas turbine performance”, Energy, 2010, 35(2), 2010, 562-570.

[20] J. H. Kim, T. S. Kim, J. L. Sohn, "Comparative Analysis of Off-Design Performance Characteristics of Single and Two-Shaft Industrial Gas", Trans. ASME J. Eng. Gas Turb. Power, 2003, 125(4), 954-960.

[21] T. S. Kim, S. T. Ro, "The effect of gas turbine coolant modulation on the part load performance of combined cycle plants-part 1: gas turbines", Proc. IMechE, Part A: J Power Energy, 1997, 211, 443-51.

[22] L. Imsland, D. Snarheim, B. A. Foss, R. Ulfsnes, O. Bolland, "Control issues in the design of a gas turbine cycle for $\mathrm{CO}_{2}$ capture", International Journal of Green Energy, 2(2), 2005, 1-15.

[23] D. Snarheim, "Modeling, simulation and control of the semiclosed $\mathrm{O}_{2} / \mathrm{CO}_{2}$ gas turbine cycle", Master's thesis, Norwegian University of Science and Technology, 2004.

[24] D. Snarheim, "Control issues in oxy-fuel combustion", Doctoral Thesis, Norwegian University of Science and Technology, 2009.

[25] D. Snarheim, L. Imsland, B. A. Foss, R. Ulfsnes, O. Bolland, "Control design for a gas turbine cycle with $\mathrm{CO}_{2}$ capture capabilities", Modeling, Identification and Control, 2006, 27, 57-66.

[26] Y. Tan, M. A. Douglas, K. V. Thambimuthu, "CO2 capture using oxygen enhanced combustion strategies for natural gas power plants", Fuel, 81 (2002), pp 1007-1016. 
Bibliografía.

[27] K. Andersson, F. Johnsson, "Flame and radiation characteristics of gas fired O2 CO2 combustion", Fuel, 86 (2007), pp 656-668.

[28] M. Ditaranto, J. Hals, "Combustion instabilities in sudden expansion oxyfuel flames", Combustion and flame, 146 (2006), pp 493-512.

[29] P. Kutne, B. K. Kapadia, W. Meier, M. Aigner, "Experimental analysis of the combustion behaviour of oxyfuel flames in a gas turbine model combustor", Proceedings of the Combustion Institute 33 (2011) 3383-3390, doi:10.1016/j.proci.2010.07.008.

[30] M. A. Nemitallah, M. A. Habib, "Experimental and numerical investigations of an atmospheric diffusion oxy-combustion flame in a gas turbine model combustor", Applied Energy 111 (2013) 401-415, http://dx.doi.org/10.1016/j.apenergy.2013.05.027.

[31] A. Amato, B. Hudack, P. D'Carlo, D. Noble, D. Scarborough, J. Seitzman, T. Lieuwen, "Methane Oxycombustion for Low CO2 Cycles: Blowoff Measurements and Analysis", Journal of Engineering for Gas Turbines and Power, 133, (2011), 061503-1-9.

[32] A. P. Shroll, S. J. Shanbhogue, A. F. Ghoniem, "dynamic-stability characteristics of premixed methane oxy-combustion", Proceedings of ASME Turbo Expo 2011, GT2011-45753.

[33] J. Ruan, H. Kobayashi, T. Niioka, "Combined effects of nongray radiation and pressure on premixed $\mathrm{CH} 4 \mathrm{O} 2 \mathrm{CO} 2$ flames", Combustion and flame 124 (2001), pp 225-230.

[34] V. Becher, S. Clausenb, A. Fateevb, H. Spliethoff, "Validation of spectral gas radiation models under oxyfuel conditions. Part A: Gas cell experiments", International Journal of Greenhouse Gas Control 5S (2011) S76-S99, doi:10.1016/j.jjggc.2011.05.005 
[35] V. Becher, J. Bohn, P. Dias, H, Spliethoff, "Validation of spectral gas radiation models under oxyfuel conditions-Part B: Natural gas flame experiments", International Journal of Greenhouse Gas Control 5S (2011) S66S75, doi:10.1016/j.ijggc.2011.05.006.

[36] D. L. Chase, P. T. Kehoe, "GE combined-cycle product line and performance", General Electric Power Systems report GER-3574G, Schenectady, USA.

[37] S. Gordon, B. J. McBride, "Computer Program for Calculation of Complex Chemical Equilibrium Compositions and Applications. I. Analysis", NASA RP1311, 1994.

[38] International Association for the Properties of Water and Steam, "Revised Release on the IAPWS Industrial Formulation 1997 for the Thermodynamic Properties of Water and Steam", 2007. Disponible en Web: www.iapws.org.

[39] IPCC, Climate Change 2007: Synthesis Report. Contribution of Working Groups I, II and III to the Fourth Assessment Report of the Intergovernmental Panel on Climate Change [Core Writing Team, Pachauri, R.K and Reisinger, A. (eds.)]. IPCC, Geneva, Switzerland.

[40] A. R. Wadia, D. P. Wolf, F. G. Haaser, "Aerodynamic design and testing of an axial flow compressor with pressure ratio of 23.3:1 for the LM2500+ gas turbine", J Turbomachinery 2002;124:331-40.

[41] A. J. B. Jackson, A. C. Neto, M W Whellens, H Audus, "gas turbine performance using carbon dioxide as working fluid in closed cycle operation" ASME 2000-GT-153.

[42] K. Jordal, O. Bolland, D Bücker, M Rost, "Gas turbine cooling model for evaluation of novel cycles", Proceedings of ECOS 2005, Trondheim, Norway.

[43] J. Ramachandran, M. C. Conway, "MS6001FA - An advanced technology 70 MW class 50/60 Hz Gas Turbine", GE Power Systems. 
Bibliografía.

[44] General Electric Power Systems, "Gas Turbine and combined Cycle products".

[45] Electric Power Research Institute, "Design Evolution, Durability and Reliability of Mitsubishi Heavy Industries Heavy Duty Combustion Turbines", 2007.

[46] F. Franco, T. Mina, G. Woolatt, M. Rost, O. Bolland, "Characteristics of Cycle Componentes for CO2 Capture", Proceedings of 8th International Conference on Greenhouse Gas Control Technologies, Trondheim, Norway, 2006.

[47] J. H. Horlock, Advanced Gas Turbine Cycles, Elsevier Science, 2003.

[48] P. P. Wahls, P. Fletcher, Gas Turbine Performance, Second Edition, Blackwell Science, Oxford, 2004.

[49] T. W. Song, T. S. Kim, J. H. Kim, S. T. Ro, "Performance prediction of axial flow compressors using stage characteristics and simultaneous calculation of interstage parameters", Proc. IMechE, Part A: J Power Energy, 2001, 215, 89-98.

[50] F. Haglind, B. Elmegaard, "Methodologies for predicting the part-load performance of aeroderivative gas turbines" Energy, 2009, 34(10) 1484-1492

[51] T. S. Kim, S. T. Ro, "The effect of gas turbine coolant modulation on the part load performance of combined cycle plants-part 2: combined cycle plant", Proc IMechE, Part A, J Power Energy 1997, 211, 453-459.

[52] F. P. Incropera, D. P. De Witt, Introduction to Heat Transfer, 3rd ed, John Wiley \& Sons, 1996.

[53] AGARD reports, "Gaseous humidity effect on components and engine performance", AGARD AR-332, 1995. 
Bibliografía.

[54] S. K. Roberts, S. A. Sjolander, "Effect of the Specific Heat Ratio on the Aerodynamic Performance of Turbomachinery", Trans. ASME J. Eng. Gas Turb. Power, 2005, 127, 773-280.

[55] J. F. Klapproth, M. L. Miller, D. E. Parker, "Aerodynamic development and performance of the CF6-6 LM2500 compressor", AIAA paper 1979-7030.

[56] J. F. Kikstra, A. H. M. Verkooijen, "Dynamic Modeling of a Cogenerating Nuclear Gas Turbine Plant-Part II: Dynamic Behavior and Control", J. Eng. Gas Turbines Power 124(3), 734-743, doi:10.1115/1.1426087.

[57] K. N. Pradeep Kumar, A. Tourlidakis, P. Pilidis, P, "Performance Review: PBMR Closed Cycle Gas Turbine Power Plant, Gas Turbine Power Conversion Systems for Modular HTGRs", Report of Technical Committee meeting held in Palo Alto, USA; November 2000.

[58] G. P. Smith, D. M. Golden, M. Frenklach,, N. W. Moriarty, B. Eiteneer, M. Goldenberg, C. T. Bowman, R. K. Hanson, S. Song, W. C. Gardiner, Jr., V. V. Lissianski, Z. Qin, available at http://www.me.berkeley.edu/gri_mech/, November 06, 2016.

[59] S. R. Turns, An introduction to combustion: concepts and applications, 2nd ed, Mac Graw-Hill Boston, 2000.

[60] H. C. Mongia, M. Vermeersch, D. D. Thomsen, T. J. Held, "A simple reactor-based approach for correlating lean blowout of turbopropulsion engine combustors", 37th AIAA/ASME/SAE/ASEE/Joint Propulsion Conference \& Exhibit 2001, AIAA paper 2001-3420.

[61] K. J. Syed, K. Roden, P. Martin, "A Novel Approach to Predicting NOx Emissions From Dry Low Emissions Gas", Journal of Engineering for Gas Turbines and Power, 129 (2007), pp. 672-679. 


\section{Bibliografía.}

[62] A. S. Feitelberg, V. E. Tangirala, R. A. Elliott, R. E. Pavri, R. B. Schiefer, "Reduced NOX diffusion flame combustors for industrial gas turbines", Journal of Engineering for Gas Turbines and Power, 123 (2001), pp. 757-765.

[63] J. Swithenbank, A. Turan, P. G. Felton, D. B. Spalding, "Fundamental modelling of mixing, evaporation and kinetics in gas turbine combustors", AGARD CP-275 (1980) Paper No. 2, 21 pp.

[64] H. C. Mongia, M. Vermeersch, T. J. Held, "A DRA modeling approach for correlating gaseous emissions of turbo-propulsion engine combustors", 37th AIAA/ASME/SAE/ASEE/Joint Propulsion Conference \& Exhibit 2001, AIAA paper 2001-3419.

[65] I. V. Novosselov, P. C. Malte, S. Yuan, R. Srinivasan, J. C. Y. Lee, "Chemical Reactor Network Application to Emissions Prediction for Industrial DLE Gas Turbine," ASME Paper No. GT2006-90282.

[66] J. Mattingly, Aircraft engine design, 2nd ed, AIAA Education Series, Reston, (2002).

[67] S. L. Bragg, "Application of Reaction Rate Theory to Combustion Chamber Analysis," Aeronautical Research Council Pub. ARC 16170, Ministry of Defense, London, England, 1953, pp. 1629-1633.

[68] D. G. Nicol, R. C. Steele, N. M. Marinov, P. C. Malte, "The Importance of the Nitrous Oxide Pathway to NOx in Lean-Premixed Combustion", Journal of Engineering for Gas Turbines and Power, 117 (1995), pp. 100-117.

[69] T. Rutar, P. C. Malte, "NO${ }_{x}$ Formation in High-Pressure Jet-Stirred Reactors With Significance to Lean-Premixed Combustion Turbines", Journal of Engineering for Gas Turbines and Power, 124 (2002), pp. 776-783.

[70] G. A. Richards, K. H. Castleton, B. Chorpening, " $\mathrm{CO}_{2}$ and $\mathrm{H}_{2} \mathrm{O}$ diluted oxyfuel combustion for zero-emission power", Proc IMechE vol 2192005 part A: Power and Energy, pp 121-126. 


\section{Bibliografía.}

[71] T. Rutar, J. C. Y. Lee, P. Dagaut, P. C. Malte, A. A. Byrne, "NOx formation pathways in lean-premixed-prevapourized combustion of fuels with carbon-tohydrogen ratio between 0.25 and 0.88", Proc IMechE vol 2212007 part A: Power and Energy, pp 387-397.

[72] H. Alkabie, "Design methods of the ABB ALSTOM POWER gas turbine dry low emission combustion system", Proc IMechE vol 2142001 part A: Power and Energy, pp 293-315.

[73] J. M. Béer, "Clean combustion in gas turbines", Journal of the Institute of Energy, 68 (1995) pp 2-10.

[74] S. M. Correa, "Carbon monoxide emissions in lean premixed combustion", Journal of Propulsion and Power Vol. 8 No. 6 (1992) p. 1144-1151.

[75] D. G. Goodwin, H. K. Moffat, R. L. Speth, "Cantera: An object-oriented software toolkit for chemical kinetics, thermodynamics, and transport processes". http://www.cantera.org, 2016. Version 2.2.1, last access November 06, 2016.

[76] T. S. Snyder, T. J. Rosfjord, J. B. Mcvey, A. S. Hu. B. C Schlein, "Emission and performance of a lean-premixed gas fuel injection system for aeroderivative gas turbine engines", Journal of Engineering for Gas Turbines and Power, 118 No. 1, (1996), pp. 38-45.

[77] G. Leonard, J. Stegmaier, "Development of an Aeroderivative Gas Turbine Dry Low Emissions Combustion System", Journal of Engineering for Gas Turbines and Power, 116 (1994), pp. 542-546.

[78] J. C. Barnes, A. M. Mellor, "Quantifying Unmixedness in Lean Premixed Combustors Operating at High-Pressure Fired Conditions", Journal of Propulsion and Power Vol.14 No.6 (1998), pp 974-980.

[79] D. E. Brandt, R. R. Wesorick, "GE Gas Turbine Design Philosophy", GE Industrial \& Power Systems, GER-3434D. 
Bibliografía. 